AFRL-IF-RS-TR-2002-19

Final Technical Report

February 2002

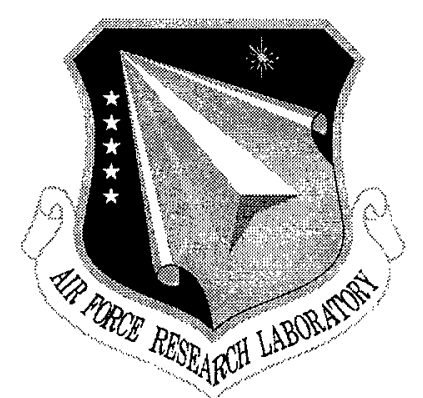

\title{
MANUFACTURING AND APPLICATIONS OF MULTISENSOR SYSTEMS
}

Boeing Phantom Works

Sponsored by

Defense Advanced Research Projects Agency

DARPA Order No. J346

The views and conclusions contained in this document are those of the authors and should not be interpreted as necessarily representing the official policies, either expressed or implied, of the Defense Advanced Research Projects Agency or the U.S. Government.

\author{
AIR FORCE RESEARCH LABORATORY \\ INFORMATION DIRECTORATE \\ ROME RESEARCH SITE \\ ROME, NEW YORK
}


This report has been reviewed by the Air Force Research Laboratory, Information Directorate, Public Affairs Office (IFOIPA) and is releasable to the National Technical Information Service (NTIS). At NTIS it will be releasable to the general public, including foreign nations.

AFRL-IF-RS-TR-2002-19 has been reviewed and is approved for publication.

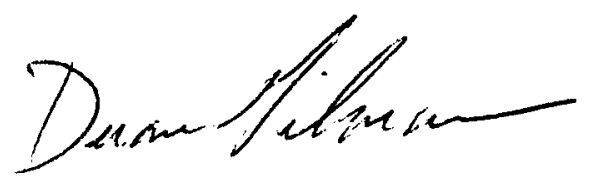

APPROVED: DUANE GILMOUR

Project Engineer

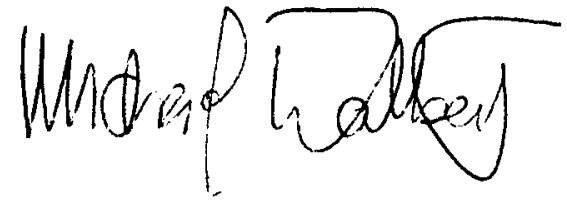

FOR THE DIRECTOR: $\quad$ MICHAEL TALBERT, Maj, USAF.

Technical Advisor

Information Technology Division

Information Directorate

If your address has changed or if you wish to be removed from the Air Force Research Laboratory Rome Research Site mailing list, or if the addressee is no longer employed by your organization, please notify AFRL/IFTC, 26 Electronic Pky, Rome, NY 13441-4514. This will assist us in maintaining a current mailing list.

Do not return copies of this report unless contractual obligations or notices on a specific document require that it be returned. 


\begin{tabular}{|c|c|c|c|c|}
\hline \multicolumn{3}{|c|}{ REPORT DOCUMENTATION PAGE } & & $\begin{array}{l}\text { Form Approved } \\
\text { OMB No.0704.0188 }\end{array}$ \\
\hline \multicolumn{5}{|c|}{ 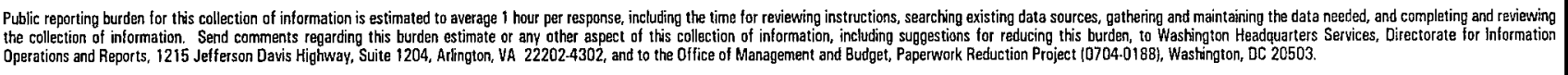 } \\
\hline 1. AGENCY USE ONLY (Leave blank) & 2. REPORT DATE & \multicolumn{2}{|c|}{ Final Apr $97-$ Feb 01} & \\
\hline \multicolumn{3}{|c|}{ MANUFACTURING AND APPLICATIONS OF MULTISENSOR SYSTEMS } & \multicolumn{2}{|c|}{$\begin{array}{l}\text { 5. FUNDING MUMBERS } \\
\text { C }- \text { F30602-97-2-0099 } \\
\text { PE }-63739 E \\
\text { PR }- \text { E117 }\end{array}$} \\
\hline Minas H. Tanielian & & & \multicolumn{2}{|c|}{ WU - 12} \\
\hline $\begin{array}{l}\text { 7. PERFORMING ORGANIZATION WA } \\
\text { Boeing Phantom Works } \\
\text { PO Box } 3999 \text {, MS 3W-81 } \\
\text { Seattle, WA } 98124-2499\end{array}$ & AND ADDRESS(ES) & & $\begin{array}{l}\text { 8. PERFORI } \\
\text { REPORT }\end{array}$ & $\begin{array}{l}\text { MING ORGANIZATION } \\
\text { MUMBER }\end{array}$ \\
\hline \multicolumn{2}{|c|}{$\begin{array}{l}\text { Defense Advanced Research Projects Agency } \\
3701 \text { North Fairfax Drive } \\
\text { Arlington, VA 22203-1714 }\end{array}$} & $\begin{array}{l}\text { AFRL/IFTC } \\
\text { 26 Electronic Pky } \\
\text { Rome, NY 13441-4514 }\end{array}$ & $\begin{array}{l}\text { 10. SPONSO } \\
\text { AGENCY }\end{array}$ & $\begin{array}{l}\text { ORING/MONITORING } \\
\text { Y REPORT NUMBER } \\
\text { RLL-IF-RS-TR-2002-19 }\end{array}$ \\
\hline $\begin{array}{l}\text { 11. SUPPLEMENTARY NOTES } \\
\text { AFRL Project Engineer: I }\end{array}$ & Gilmour, IFTC, 315-330 & & & \\
\hline \multicolumn{3}{|c|}{$\begin{array}{l}\text { 12a. DISTRIBUTION AVAILABILITY STATEMENT } \\
\text { Approved for public release; distribution unlimited. }\end{array}$} & \multicolumn{2}{|c|}{ 12b. DISTRIBUTION CODE } \\
\hline \multicolumn{5}{|c|}{$\begin{array}{l}\text { 13. ABSTRACT (Maximum 200 words) } \\
\text { Current Flight Loads Testing technology of a new airplane wing includes a plastic tube system with remotely located } \\
\text { pressure sensors, discrete electronics, and pneumatic system control. This approach is cumbersome to install, expensive to } \\
\text { operate, and lacks the desired accuracy. This effort developed a MEMS-based, pressure sensor tape, which provides a } \\
\text { multitude of "smart" pressure sensors at the point-of-use, through integration of the sensors and their corresponding } \\
\text { electronics on a MCM. To accomplish this goal, research was focused on the development of the following: a) a standard } \\
\text { network interface protocol capable of handling large numbers of sensors, b) a low-profile, high accuracy MEMS pressure } \\
\text { sensor, c) innovative packaging techniques, including tape design and packaging of the MEMS sensor with its associated } \\
\text { electronics on a high density multichip module substrate, and d) thin protective coatings which will be used to protect the } \\
\text { various devices and components from adverse environmental conditions and provide an aerodynamically smooth surface. } \\
\text { Validation of these developments and the objective of attaining an accuracy within } 0.1 \% \text { of the full scale pressure reading } \\
\text { (roughly } 10 \mathrm{X} \text { better than the state-of-the-art), was realized both in the laboratory and flight tests on both military and } \\
\text { commercial airplanes. }\end{array}$} \\
\hline \multirow{2}{*}{\multicolumn{4}{|c|}{$\begin{array}{l}\text { 14. SUBJECT TERMS } \\
\text { Microelectromechanical Systems (MEMS), Flight Testing, Multichip Module (MCM), pressure } \\
\text { sensor, pressure belt, smart sensor, reliability without hermeticity (RWOH), conformal } \\
\text { coatings }\end{array}$}} & 15. NUMBER OF PAGES \\
\hline & & & & \begin{tabular}{|c|}
176 \\
16. PRICE CODE
\end{tabular} \\
\hline \multicolumn{2}{|c|}{\begin{tabular}{|l|l|} 
coatings \\
17. SECURITY CLASSIFICATION & $\begin{array}{l}\text { 18. SECURITY CLASSIFICATION } \\
\text { OF THIS PAGE }\end{array}$ \\
\end{tabular}} & $\begin{array}{l}\text { 19. SECURITY CLASS } \\
\text { OF ABSTRACT }\end{array}$ & & 20. LIMITATION OF ABSTRAC \\
\hline UNCLASSIFIED & UNCLASSIFIED & UNCLA: & & UL \\
\hline
\end{tabular}


1.0 EXECUTIVE SUMMARY

2.0 PROGRAM OBJECTIVES AND APPROACH

2.1 Overview

2.2 System Requirements

2.3 System Architecture

2.4 Design Strategy

3.0 TRANSDUCER-TO-BUS INTERFACE MODULE DEVELOPMENT 9

3.1 MEMS Sensor Development 9

3.2 Electronic Circuitry Development 11

3.2.1 TBIM Development $\quad 11$

3.2.2 Lab Test and Calibration 16

$\begin{array}{ll}\text { 4.0 PRESSURE BELT TRANSDUCER BUS CONTROLLER } & 19\end{array}$

4.1 TBC Hardware Architecture $\quad 19$

4.2 TBC Software Architecture $\quad 20$

5.0 PACKAGING TECHNOLOGY DEVELOPMENT 22

5.1 MEMS Device Packaging 23

5.2 Electronic Component Packaging $\quad 28$

5.3 Multichip Module Packaging $\quad 29$

5.4 Protective Coatings Development $\quad 32$

5.4.1 Thermal Dynamic Analysis Of The Modified Siloxane Elastomers 33

5.4.2 Chemical Resistance Evaluation 36

5.4.3 Mechanical Properties Characterization 38

5.5 Pressure Belt Segment Packaging 43

6.0 FLIGHT TESTING

7.0 SYSTEM INTEGRATION AND DEPLOYMENT ACTIVITIES 62

8.0 CONCLUSIONS AND RECOMMENDATIONS FOR FUTURE WORK 65

APPENDIX A: Related Publications And Conference Presentations 67

$\begin{array}{ll}\text { APPENDIX B: Patents } & 142\end{array}$ 


\section{LIST OF FIGURES}

2.1 Standard random vibration test curves for equipment installed in fixed-wing 5 aircraft with turbojet engines

2.2 Overall system architecture of the pressure belt 6

2.3 A photograph of a 777-100 wing during flight loads testing 7

3.1 Schematic representation of the redesigned MEMS pressure sensor 9

3.2 Photographs of redesigned pressure sensor, (a) front side, (b) back side $\quad 10$

3.3 Specification sheet for the redesigned sensor 11

3.4 TBIM block diagram 12

3.5 The discrete version of the TBIM 13

$\begin{array}{lll}3.6 & \text { Analog ASIC Block Diagram } & 14\end{array}$

3.7 Prototype PWB version of the analog ASIC and its die form 14

$\begin{array}{ll}3.8 & \text { Block diagram of the digital ASIC } \\ 3\end{array}$

3.9 Photographs of the digital board and the digital ASIC 16

3.10 A photograph of the discrete version of the TBIM using the packaged ASIC die 16

$\begin{array}{lll}3.11 & \text { Surface fit of the pressure channel output vs a standard as a function of } & 17\end{array}$ temperature using the TBIM electronics

3.12 Surface fit of the temperature channel output vs a standard using the TBIM 18

4.1 The architectural relationship between the NCAP, the TBC and the TBIMs 19

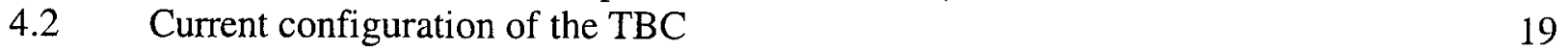

4.3 Current implementation of the NCAP, which will be used for Flight Testing 20

4.4 Diagram showing the electronic functionality of the TBC 20

$\begin{array}{ll}4.5 & \text { A block diagram of the TBC software architecture }\end{array}$

5.1 Schematic: 23

(a). Drawing (top view) of the production pressure belt showing the various parts

(b). Representation of the pressure belt cross-section

5.2 The TBIM installed on the BBJ for flight testing 24

5.3 Flight test data showing the failure of the MEMS sensors 25

$\begin{array}{ll}5.4 & \text { SEM Micrographs of the fractured diaphragm in different magnifications } \\ 5\end{array}$

5.5 A drawing of the stainless steel cap used for protecting the MEMS device from 27 direct impact of foreign objects

5.6 Results of laboratory experiments, testing the integrity of the MEMS protective 27 shield:

(a) Completely ruptured diaphragm due to water jet exposure

(b) Damaged BBJ test samples (hairline crack, too fine to be seen in this magnification)

(c) The protected sensor after removing the lid, showing no damage

5.7 A photograph of a TBIM MCM with the metal shield installed 28

5.8 Photograph of the assembled analog MCM module used for flight testing 29 (without the protective coatings applied).

5.9 A cross-sectional drawing of the fairing design 30

5.10 A picture of the analog pressure belt used for flight testing 30

5.11 The layout of the digital TBIM MCM and a photograph of an assembled part 31 (without the protective coatings applied) 


\section{LIST OF FIGURES (CON'T)}

5.12 DSC comparing the curing rate of Q1-4939 using different catalysts 34

5.13 DSC curves comparing the effect on the curing temperature of Q1-4939 using 35 different PC085 concentrations and also the effect of adding a filler

5.14 Q1-4939 swelling vs. filler loading percentage 36

$\begin{array}{lll}5.15 & \text { Material swelling vs. filler loading for a room temperature cured Q1-4939 resin } 37\end{array}$ (after immersion in jet fumes for 5 minutes) with and without a filler

5.16 Storage modulus change of Q1-4939 as a function of the percentage of filler loaded into the resin system. SC stands for the silane coupling agent used to improve adhesion

5.17 Moduli comparison on room temperature - cured Q1-4939 resin before and after soaking in gasoline for 5 minutes

5.18 Moduli comparison on high temperature curable resin with filler loading before and after soaking in gasoline for 5 minutes

5.19 Surface insulation resistance of candidate coatings vs. aging time under $85 \%$ relative humidity $@ 85^{\circ} \mathrm{C}$ under $160 \mathrm{~V}$ bias (in a Triple Track Test configuration)

5.20 DSC results on samples with and without $80^{\circ} \mathrm{C} / 15$ minute post cure 42

5.21 Schematic of segment design showing the various features of the pressure belt 43

5.22 Photograph of an assembled pressure belt segment 44

6.1 Photographs of the 737-700 wing, which was undergoing flight loads testing 45

6.2 Photograph of the pressure belt attached to the belly of the 757-300 46

6.3 The side-by-side installation of the pressure tube and the pressure belt 47

6.4 Routing of the electrical and pneumatic connections in the landing gear bay of 47 the 757

6.5 Flight test data taken on the 757-300 airplane 48

6.6 The percentage difference between the MEMS sensor readings and the 49

6.7 Photograph of the Boeing Business Jet ready for flight testing 49

6.8 Photograph of the BBJ slat and the MEMS MCM installation 50

6.9 Photograph of final installation on the BBJ forward slat 51

6.10 Pressure measurements as a function of time for a "wind-up turn" of the BBJ 51

6.11 Pressure measurements as a function of time for a "roller-coaster" maneuver 52

6.12 Calculated air speed as a function of location (cord fraction) on the BBJ wing 53

6.13 Photograph of the 767-400 aircraft and a close-up of the module installation 54

6.14 Calibration curve comparison equal to or above $10^{\circ} \mathrm{C}$ after long duration test 55

6.15 Calibration curve comparison equal to or below $0^{\circ} \mathrm{C}$ after long duration test 56

6.16 Random vibration spectrum with 41-g peak values and an RMS value of 14-g 57

6.17 Sensor calibration curve before and after 3-axes vibration testing (15 min/axis) 57

6.18 The Navy F-18E3 undergoing flight tests at NAWC at Patuxent River 58

6.19 Installation of the pressure belt on a F-18E3 at Patuxent River Naval Station 58

6.20 A close-up of the pressure belt installation on the F-18E3 59

6.21 Pressure measurement of the MEMS sensor compared to a reference pressure, $\quad 60$

6.22 A comparison of the reference pressure to the four MEMS channels available 60 
Figure

\section{LIST OF FIGURES (CON'T)}

6.23 An expanded portion of the comparison of the reference pressure to one of the 61 MEMS sensors

7.1 An multisensor instrumentation system using conventional point-to-point interconnections

7.2 The architectural relationship between the NCAP, the TBC and the TBIMs 


\section{LIST OF TABLES}

2.1 Estimates of the various intrinsic sources of error

Page

5.1 Results from the optimization experiments using different catalysts

4

5.2 Room temperature cured sample properties for various filler combinations

36

6.1 Pre-flight and post-flight average pressures measured on the BBJ airplane

39

6.2 F-18E3 flight conditions

53

59 


\section{AKNOWLEDGEMENTS}

This program was carried out by a team consisting of workers at various institutions. Here, we would like to acknowledge their contributions and creativity.

There were three different teams from The Boeing Company

Phantom Works

Namsoo Kim

Mark Chisa

Jean Nielsen

$\mathrm{Kin} \mathrm{Li}$

Nelli Amirgulyan

Linda Branson

Colleen Littlejohn

\section{Endevco Corporation}

Alex Karolys

Fernando GenFong

Ron Poff

Craig Evensen

George Pender

Georgia Institute of Technology

C. P. Wong

Jiali Wu

The U.S. Navy, Naval Air Warfare Center (Patuxent River Station, MD)

Bill Reinking

Marvin Murray

Ray Faulstich
Flight Test/Validation

John Stice

Dave Smith

Mark Holland

Larry Malchodi

Lee Eccles

Hung Mach

Rod Juelfs

Mark Slack

Wayne Catlin

Dan Patke (F-18, Pax River)
Gary Nelson

Scott Billings 


\subsection{EXECUTIVE SUMMARY}

The current technology used in Flight Loads Testing includes an extensive network of thin plastic tubes, which are routed to remotely located pressure detectors using a pneumatic control system. The system is labor-intensive to install, expensive to operate, and lacks the desired accuracy.

The overall goal of this program was the development of a multi-sensor pressure belt system, which uses a multitude of "smart" pressure sensors at the locations of interest. The sensor is rendered "smart" through integration with its associated electronics on a high-density multi-chip module (MCM) at each node. Furthermore, by providing data processing capability on the module, the overall accuracy of the system is dramatically enhanced and a bus architecture is enabled. Our development goal was to design a system that is easy to install and operate, having more than $10 \mathrm{X}$ better accuracy than the state-of-the-art technology.

The design strategy adopted early in the program was to make the system modular. All parts were designed and tested in a brass-board or prototype configuration first. This allowed us to debug each part separately, prior to system miniaturization. Discrete versions of each Application Specific Integrated Circuit (ASIC) and of the fully functional Transducer to Bus Interface Module (TBIM) were built, tested, and debugged, using conventionally packaged parts. However, the design of the discrete/prototype hardware took into consideration inherent design limitations present in the integrated/miniaturized versions of the same hardware. This also allowed the development of the control software using the discrete version and thus minimized program risk. Some of the detailed program objectives were to develop:

(a) A low-profile, high accuracy MEMS pressure sensor

(b) The associated electronics to render this sensor "smart"

(c) Innovative packaging techniques, including tape design and integration of the MEMS sensor and its associated electronics on a high density multichip module substrate

(d) Thin protective coatings to protect the various devices and components from adverse environmental conditions

(f) A network interface protocol capable of handling large numbers of sensors

Validation of these developments was done through a series of laboratory tests. In addition, Boeing used mainly internal funds to put the hardware through several Flight Tests using both Commercial and Military Airplanes of The Boeing Company. The objective of attaining an accuracy within $0.1 \%$ of the full scale pressure reading (roughly $10 \mathrm{X}$ better than the state-of-theart), was realized both in laboratory and flight tests using two different versions of the hardware. Also, this program demonstrated for the first time, a networked MEMS-based multi-sensor system suitable for a variety of aerospace applications.

There were also many challenges encountered during the course of the program. The packaging of the devices, both for the MEMS sensor and the electronics had to be developed so that it was suitable for the flight test environment. Specialized coatings had to be used to protect the devices and the MCM from adverse environmental effects because conventional packaging did not meet the low profile requirement of the pressure belt. Furthermore, the MEMS sensors had to be packaged in a robust configuration while still maintaining a low profile. 
One of the goals of the program was to use an IEEE standard interface for the multisensor bus. Unfortunately, the appropriate standard, IEEE P1451.3 has not yet been established, although it will most likely be finalized later this year. Thus, we had to establish an intermediate standard based on an RS-485 bus to meet the program objectives.

The technology development team consisted of researchers from three institutions: The Boeing Company, Endevco Corporation, and the Georgia Institute of Technology.

The responsibilities of the Boeing team members were: requirements definition, the overall system design and test as well as that of various hardware subassemblies, including the digital functionality on the TBIM, the Transducer to Bus Controller (TBC), the control software, the TBIM packaging, the TBIM integration using ASICs, and flight test of prototype hardware on various platforms.

The responsibilities of the Endevco team members were: redesign and optimization of the MEMS sensor, test of the MEMS sensor, development of the analog front-end in the TBIM, design of the digital bus interface in the TBIM, test of various subassemblies, and test of various brass-board versions of the hardware.

The responsibilities of the Georgia Tech team members were the evaluation and recommendation of suitable protective coatings for the pressure belt hardware in an aerospace environment.

Finally, the success of the program depended to a large extent on team spirit and clear focus of the objectives. This was achieved by bringing together a team of innovators and users, as well as those responsible for the commercialization of the final product. 


\subsection{PROGRAM OBJECTIVES AND APPROACH}

\subsection{Overview}

The aerodynamic performance of a new airplane wing is initially modeled using Computational Fluid Dynamics techniques. These simulations are then compared to data obtained in a Wind Tunnel using a scaled down model. Ultimately, the design must be tested and verified in a real operational environment before it is validated and certified. One of the critical parameters measured during flight testing of a new design are the loads (forces) present at different locations on the airplane wing. The current technology used in Flight Loads Testing includes an extensive network of thin plastic tubes (approx. 1/8" diameter), which are glued to the surface of the wing. A tiny hole is punched in these tubes at the location where the pressure (force/area) needs to be measured. These tubes are routed to remotely located pressure detectors inside the wing, using a pneumatic system control. This system is labor-intensive to install, expensive to operate, and lacks the desired accuracy.

The overall objective was to replace this system with a set of "smart" sensors at the locations of interest, without having to replace the tube-based system with a bunch of wire bundles. This approach improves the system performance because the sensor is positioned at the location of interest, instead of being as much as 20-30 feet away. Furthermore, this new system has much better dynamic response because it is not susceptible to the filtering action present in the long, thin tube system.

The overall approach was to make the sensors "smart" through integration of the sensor with its associated electronics at the point-of-use. Combining this with some signal processing capability, a local correction engine, and a bus interface allowed each of the "smart" sensor modules to connect to a bus, thus eliminating the need for any discrete wiring.

At the program start, we decided that the architecture of the system had to be modular. This would allow the pressure belt to be made of smaller units, which would be easier to build, test, and handle. We also decided that before the design was miniaturized for the flight test environment, it was essential that each subassembly be built using conventional parts and packaging techniques. Shortly after we started designing the brass-board versions of the hardware, it became apparent that there was an error in our approach. The reason was that we were using the best commercial off-the-shelf (COTS) to achieve the desired functionality. Since a lot of these parts could not be used in the miniaturized version using ASICs, we decided to change course and make the design of the brass-board hardware subject to the same limitations as the miniaturized version. This allowed for a direct comparison between the discrete (brassboard) version of the hardware and the integrated (miniaturized) version in terms of functionality, software development, and debugging capability.

One of the strengths of this program and a major contributor to its success was the fact that the program requirements were defined at the beginning of the program. These requirements helped focus the team efforts on a common set of goals and defined clearly the criteria for success. 


\subsection{System Requirements}

The following requirements were defined early on and guided the development of the program:

- Thin polymeric tape (belt) with embedded conductor lines

- Total height: $0.1 "$

- Separation between sensors: 2"

- Pressure range: 0-15 psi (absolute)

- Overall accuracy: $0.1 \%$ (with temperature compensation)

- Sensor scan rate: 20 samples/sec/sensor

- Operating range: -55 to $80^{\circ} \mathrm{C}, 100 \%$ relative humidity

- Vibration and Shock: RTCA/DO-160C, Section 8.5.2, Figure 8-1, reproduced in Figure 2.1

- Chemical environment: Airplane fuel, hydraulic fluid, solvents

- Cost: $\$ 100 /$ sensor module, $\$ 50 \mathrm{~K}$ for a 200 -sensor belt

- Installation: Five working days per airplane (1000-2000 sensors)

- Sensor data network: IEEE P1451.3 standard or equivalent

The requirement that the total height of the pressure belt be $\leq 0.1$ " is based on aerodynamic reasons. To avoid disturbing the airflow in the boundary layer right above the wing, it is necessary for the belt to be much thinner than that layer. The thickness of the boundary layer is a function of various parameters, one of which is the Reynolds number of the flow stream. Since typically there is a layer of adhesive underneath the belt, the devices present on the "smart" sensor module cannot be packaged nor can they be macroscopic. This led to the choice of a MEMS-type pressure transducer. There are only a handful of companies that produce a MEMStype transducer as part of their product line. Of these, the requirement of having an accuracy of $0.1 \%$ in the pressure measurement over the whole temperature range of operation on the system level translated into a transducer product that had an intrinsic error margin lower than $0.1 \%$. Endevco Corporation was chosen as the transducer provider because they have consistently delivered high quality products to The Boeing Company in past programs. Furthermore, their willingness to license and commercialize the technology was also a key element.

An analysis on an existing Endevco product was conducted early in the program to assess the various sources of error we may encounter to help us optimize the design to achieve our set goal. The estimated sources of error that can contribute to the overall accuracy of the pressure belt measurement are given in Table 2.1. This analysis does not take into account external sources of error such as temperature and stress, which are addressed through other means.

Table 2.1. Estimates of the various intrinsic sources of error

\begin{tabular}{|l|c|}
\hline Transducer Hysteresis & $\pm 0.040 \%$ \\
\hline TransducerNon-Repeatability & $\pm 0.040 \%$ \\
\hline 12 bit A/D & $\pm 0.024 \%$ \\
\hline Front-End Amplifier & $\pm 0.012 \%$ \\
\hline Noise & $\pm 0.005 \%$ \\
\hline Accuracy of Temperature Measurement & $\pm 0.004 \%$ \\
\hline Worst Case Total & $\pm 0.125 \%$ \\
\hline Root-Sum med-Squared Total & $\pm 0.063 \%$ \\
\hline
\end{tabular}




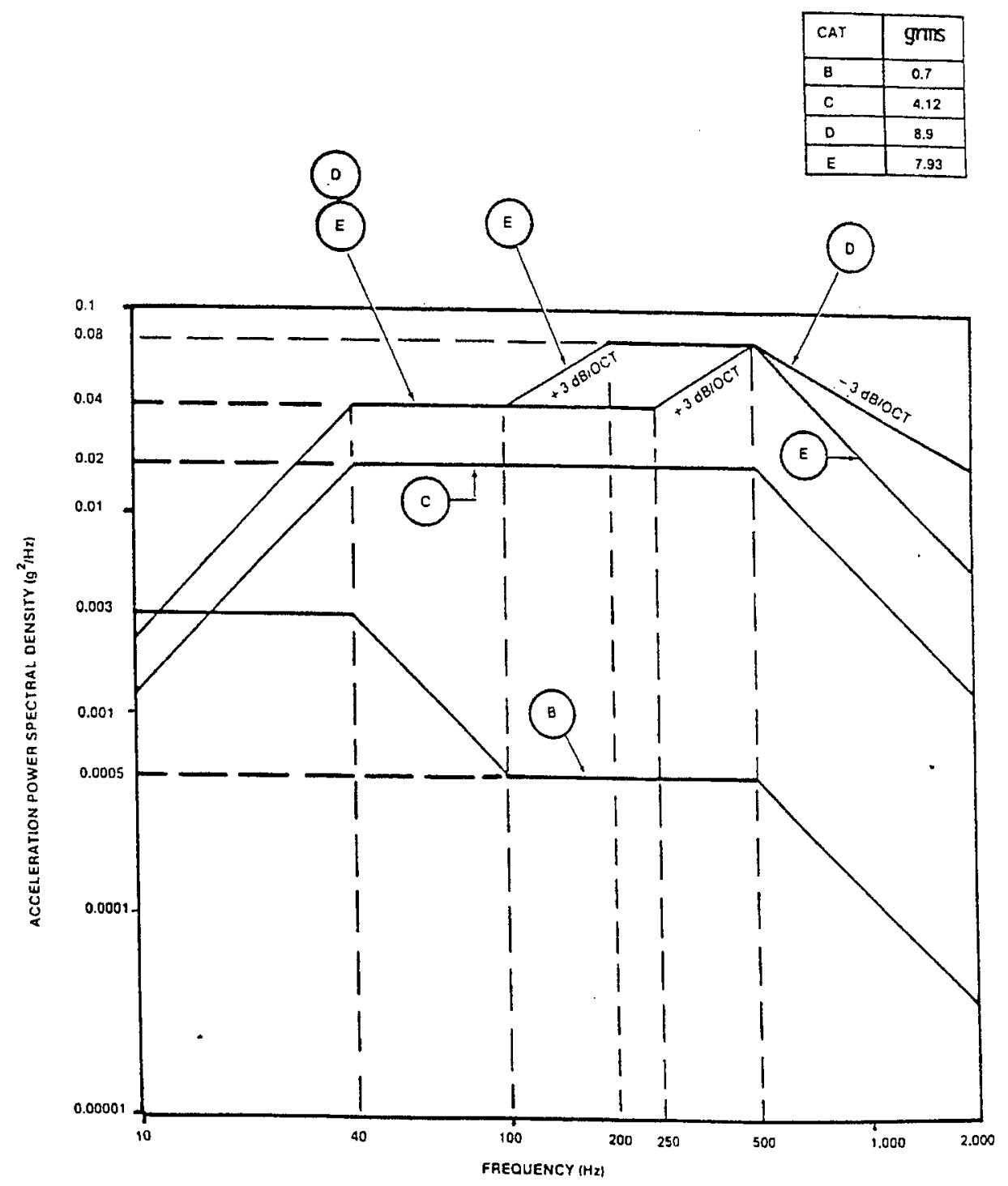

NOTE: All slopes are $\pm 6 \mathrm{~dB} / \mathrm{OCT}$ except as noted on curves $D$ and $E$.

Figure 2.1. Standard random vibration test curves for equipment installed in fixed-wing aircraft with turbojet engines.

Although one of our goals at the outset was to reduce the cost of the hardware as much as possible, we were fully aware that this was only a secondary goal. Even if the hardware costs were comparable or perhaps somewhat higher than the current implementation, the largest cost savings are in the recurring labor costs, every time a loads survey is done in flight testing. We estimate that there will be as much as a $5 \mathrm{X}$ improvement in the installation costs per airplane, per test. It has been estimated that over the lifetime of the program this will save as much as $\$ 30 \mathrm{M}$ over the current method. Furthermore, the improved pressure measurement capability will lead to better aerodynamic designs and better on-time deliveries, which also translates to many millions of dollars. Finally, the modular approach adopted is suitable for a variety of aircraft and eliminates many "custom" features of the legacy system. This need for modularity led us to the system design and architecture discussed in the next section. 


\subsection{System Architecture}

The overall system architecture is shown in Figure 2.2. One can easily see the modularity in the system design. The pressure belt is made of a variable number of segments, each segment having as many as six "smart" transducer modules on it. The "smart" transducer modules are referred to as TBIMs (Transducer to Bus Interface Modules) a term derived by the IEEE P1451.3 terminology. More information regarding the architecture is given in reference 9. Details regarding the IEEE standard are given at http://www.ic.ornl.gov/p1451/p1451.html.

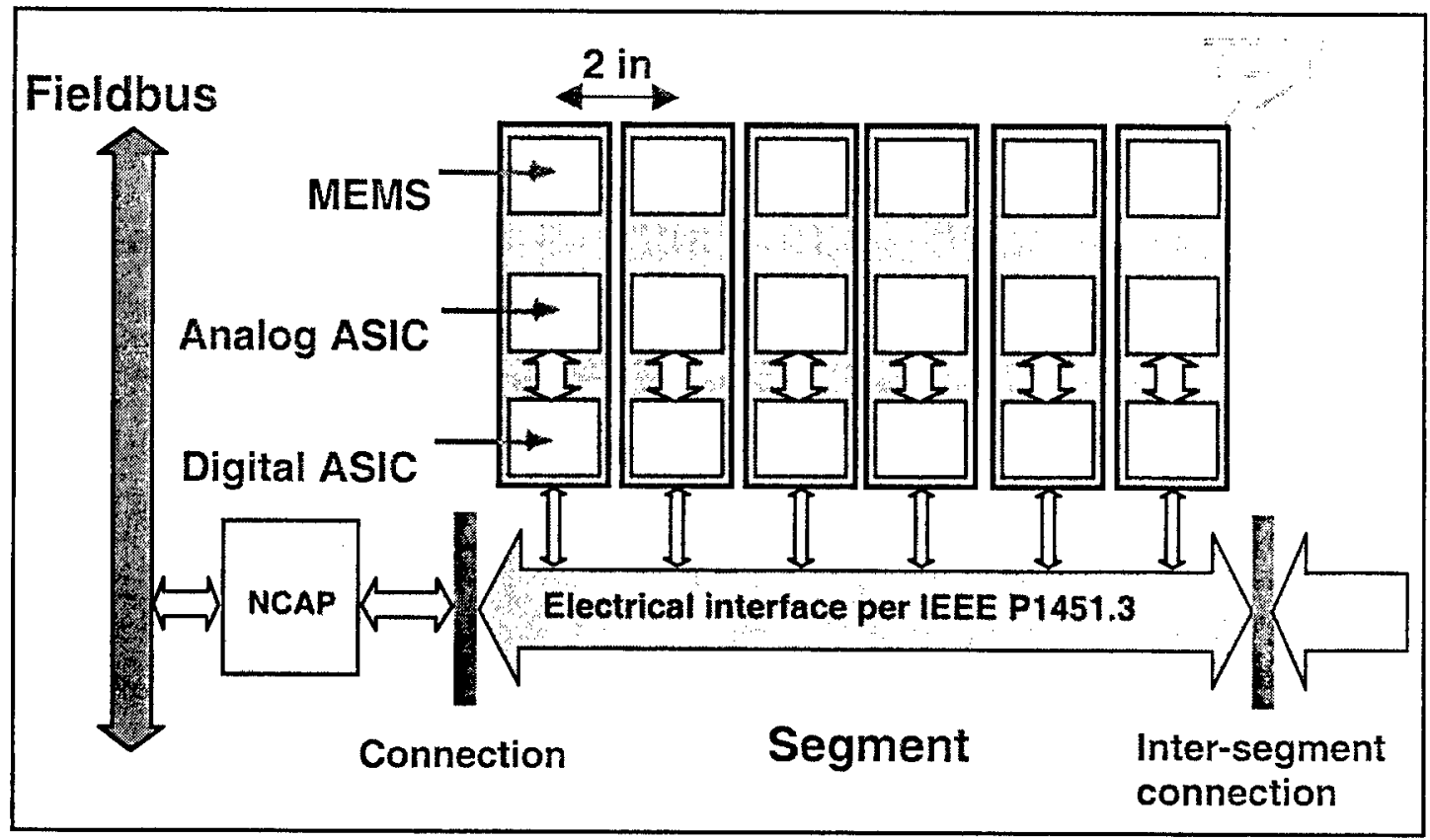

Figure 2.2. Overall system architecture of the pressure belt.

The final TBIM design has a MEMS pressure sensor, an analog ASIC, a digital ASIC, an EEPROM, a SRAM and 37 passive components (resistors and capacitors). The TBIM provides the following functions:

- Signal conditioning for a number of different types of transducers such as piezoelectric, piezoresistive, variable capacitance, and strain gauge with 4 automatically selectable antialiasing filters

- Programmable sampling rate

- Analog and digital (12 bit) output

- Correction of digital output

- Sensitivity, linearization, zero compensation

- Transducer electronic data sheet (TEDS)

- Comprehensive self test

- Digital Bus Interface 
The baseline pressure belt design accommodates up to 127 TBIMs on a single Transducer Bus Controller (TBC). The number of TBIMs used is a function of the bus clock, sampling frequency, the number of measurements taken per unit time, and the number of data channels used. These parameters are variable and can be altered depending on need.

In practice, a pressure belt could be as long as $21+\mathrm{ft}$ but its thickness has to be less than $0.1 "$. To make such an object manufacturable, easy to use, and versatile it was determined that it had to be modular, i.e., made in segments that could be put together to achieve various lengths. The reason is the length of the pressure belt needs to be longer closer to the fuselage and shorter as it approaches the wing tip. Figure 2.3 shows an airplane wing of a 777-100 during the flight loads testing phase of the airplane development cycle, fitted with conventional plastic tube pressure belts. One can clearly see that length of the pressure belts shrinks as one moves away from the fuselage. There is also another set of pressure belts on the underside of the wing, on the nacelles, and various other locations of interest.

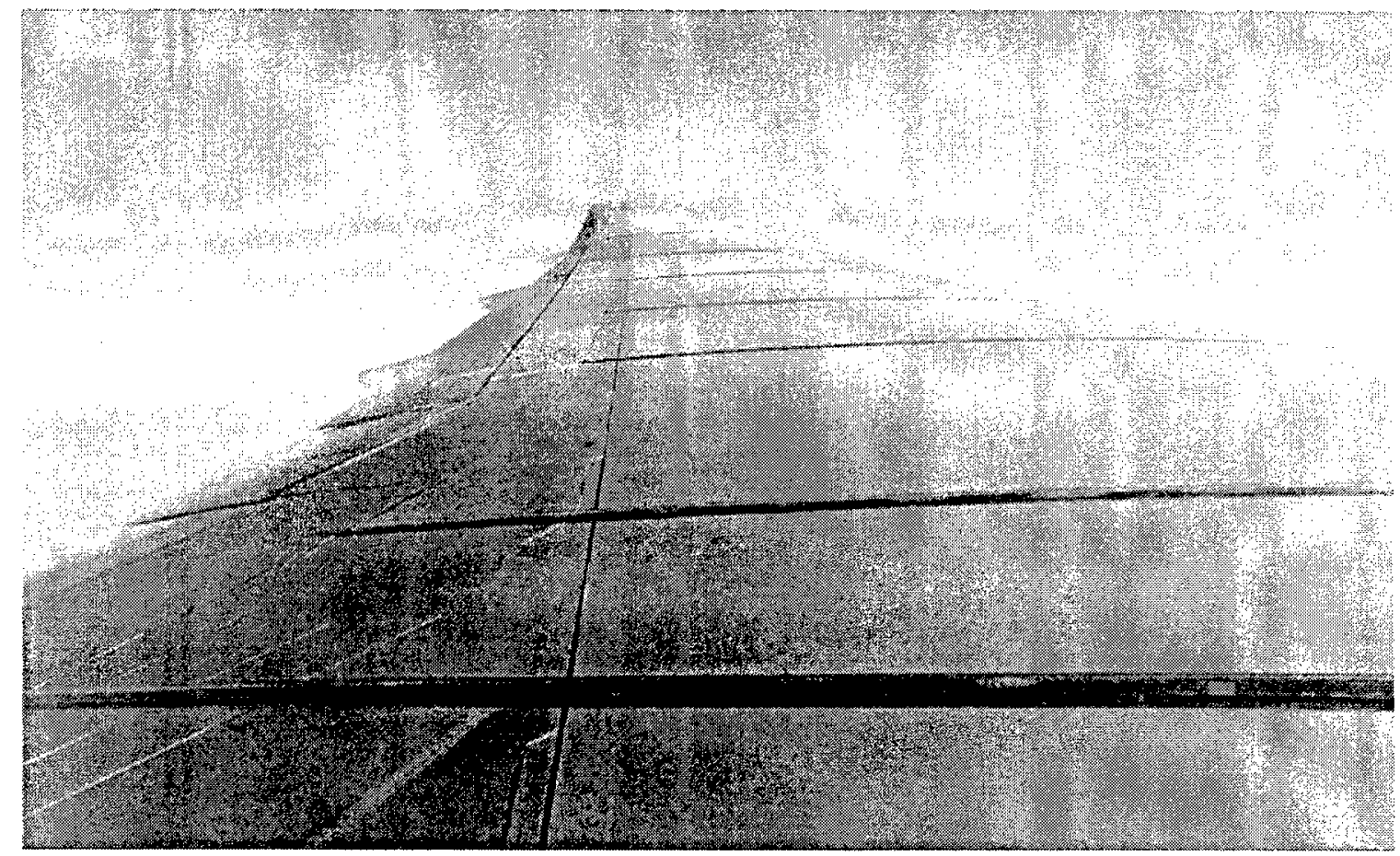

Figure 2.3. A photograph of a 777-100 wing during flight loads testing.

Another fact that also contributed to the choice of a modular approach is that the pressure measurements need to be closely spaced as one approaches the leading edge of the wing i.e., every 2 inches, while at the trailing edge, this is less important as the pressure gradients become less pronounced. This could be accomplished easily in our approach by leaving certain TBIM locations on a specific segment unpopulated for segments in the trailing end of the belt. Since all the TBIMs are connected to the bus in parallel fashion, this can be easily achieved by not fully populating the segments toward the trailing edge of the wing.

The final block of the pressure belt is the Transducer to Bus Controller (TBC). The TBC is essentially the "traffic cop" for the pressure belt. It initiates the measurement cycle and does the 
"housekeeping". The TBC is part of the Network Capable Application Processor (NCAP), which provides additional processing capability and an interface to the Fieldbus that networks the various NCAPs with each other. The TBC and the NCAP are not part of the pressure belt that resides on the wing surface, although they may be in close proximity. Ultimately, the information will be transferred to a flight test computer inside the cabin.

\subsection{Design Strategy}

The overall approach was to design and build prototype versions of all the hardware prior to designing the integrated versions. This allowed for the use of more macroscopic hardware, which easily accommodated cuts and jumpers. It also allowed for changing of various components (for instance, capacitors or resistors) in an easy fashion. Having this capability is very important for analog designs. It also facilitated the development and debugging of the system software and test of the software on these prototype versions. The result was the development of two distinct types of pressure belt hardware. The first one, which we will refer to in the later sections as the "analog" version, was primarily used to evaluate the packaging technology, the materials, and processes in a realistic environment (flight). The second one is referred to as the "digital" version. This was used for verifying and developing the full functionality of the pressure belt in the laboratory. The analog belt had the form and fit of the "final" or "production" configuration but it did not have the full functionality of that version. On the other hand, the digital version had the full functionality of the final belt but not the form and fit. This approach allowed separate testing of each configuration, thus reducing risk in the program. The digital version was designed in two passes. In the first one, the functions of the analog and digital ASIC were done on separate boards and the function of the TBIM in yet another board. In the second pass, the analog board was reduced to an ASIC as was the digital board. The two packaged ASICs were then combined on a single board to mimic the functionality of the TBIM. In the final version, this second pass digital version was reduced to an $\mathrm{MCM}$ where all the devices packaged were bare chips and thus had the highest level of integration possible.

In the ensuing sections, we will discuss in some detail the development activities related to the various components of the TBIM, which includes the sensor and the functionality resident in the ASIC chips and the TBC, which controls the bus. This will be followed by a discussion of the packaging technology used for the sensor, the MCM used for miniaturizing the TBIM and assembling its components, the protective coatings employed in lieu of conventional packaging, and the belt assembly. Finally, we will discuss the various flight tests performed on airplanes of The Boeing Company to verify that the overall design is flightworthy and that it meets the objectives set forth at the beginning of this program. 


\subsection{TRANSDUCER-TO-BUS INTERFACE MODULE DEVELOPMENT}

As mentioned previously, the TBIM provides a variety of electronic functions at the sensing location. Its role is to collect, process and communicate data to the NCAP and ultimately to the flight test computer. In order to meet this objective, the TBIM has to measure pressure and temperature, amplify the signal, filter the signal, correct the pressure reading for temperature variations, and finally broadcast the information via a bus interface. In the next few sections we discuss the TBIM electronics.

\subsection{MEMS Sensor Development}

In order to reduce risk in the program, our strategy was to start with an existing sensor that had the desired accuracy but did not necessarily meet the form factor requirements. The transducer chosen for modification was the Endevco 8515, a micro-machined Si sensor. This Endevco sensor was evaluated early in the program and it was flown in a standard packaged configuration on a 737-600 aircraft using a crude precursor to the pressure belt. A short discussion of this test is given in Section 6.0, Flight Testing.

The transducer uses an etched Si membrane (with a thickness of $\sim 1 \mu \mathrm{m}$ ) as the pressure sensing diaphragm. On the backside of the diaphragm, there is a fully active Wheatstone bridge of resistors formed using ion implantation. Since $\mathrm{Si}$ is piezoresistive, these resistors act as the strain sensing element of the transducer. This diaphragm is mounted on a second piece of $\mathrm{Si}$ which has a cavity etched in it and the two pieces are joined together in a hermetic fashion using a glass sealing. The sealing is done in high vacuum, so that the sealed vacuum cavity can be used as an absolute pressure reference. The sensor is rectangular in shape with $0.049^{\prime \prime}$ width, 0.067 " length, and 0.016" in height. Finally, the support part is etched to create feedthroughs that reach the backside of the Si membrane. The piezoresistive elements are routed to these feedthroughs and are brought out from the back side of the transducer. The transducer is shown schematically in Figure 3.1.

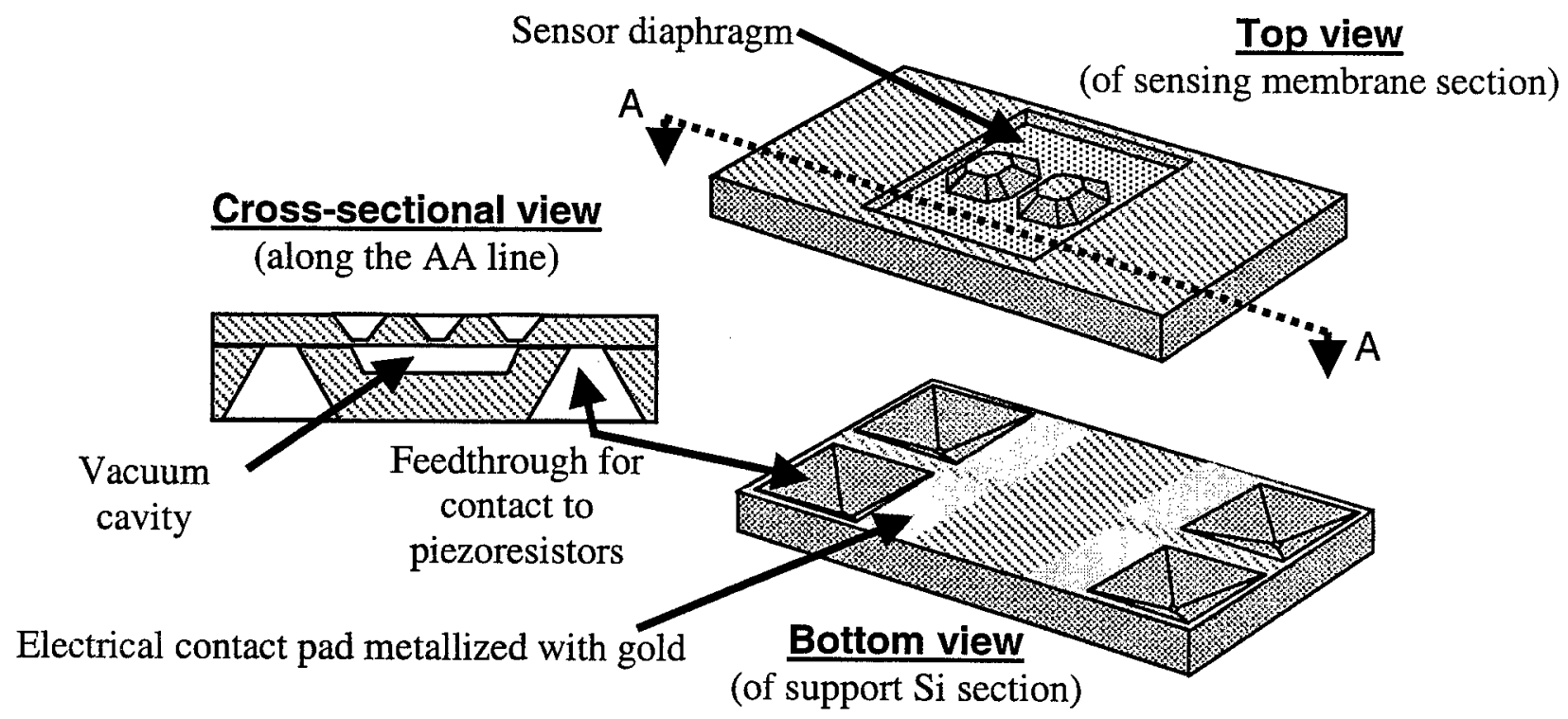

Figure 3.1. Schematic representation of the redesigned MEMS pressure sensor. 
The diaphragm used was derived from the standard Endevco 8515 transducer. The standard transducer uses aluminum trimming resistors for balancing the Wheatstone bridge on the diaphragm (for null offset), an operation done at the packaging level. Since this is not possible with the current sensor configuration (no package), the piezoresistors had to be redefined with much higher accuracy. The final fine trimming adjustment in our case is done electronically in the TBIM analog front end.

The other major difference between the existing transducer and the current configuration related to the reference support. The existing part was designed to be wirebonded to supports inside the package. Since there was a need for a much more robust bonding approach, the support section (also made of $\mathrm{Si}$ ) was redesigned to allow flip chip mounting of the MEMS assembly to the silicon MCM using solder bumps or conductive epoxy.

The electrical contact of the standard sensor was made through pads on the side of the sensor diaphragm section. In the redesigned sensor, this is achieved with feedthroughs that go through the support section and terminate on the backside of the diaphragm section. These feedthroughs are etched using an anisotropic Si etch, which results in a pyramidal shape. Finally, the contact is made using a thin film of gold that is sputtered inside the feedthrough and forms a pad for mounting on the backside of the bottom (support) section of the sensor.

The support section redesign was designed to maintain the lowest possible profile while keeping the sensor interconnection robust. This was of course very important, since only minimal protection for the sensor could be provided, due to the thickness requirements. The pressure sensors were characterized within the operating temperature range $\left(-55\right.$ to $\left.125^{\circ} \mathrm{C}\right)$ in order to compensate for the inaccuracies in the electronics firmware. Photographs of the front and back surface of the actual manufactured sensor are shown in Figure 3.2.

(a)

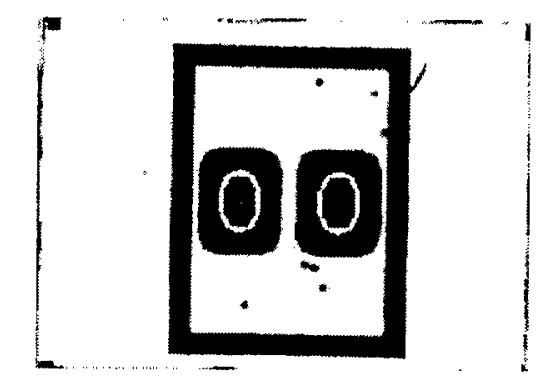

(b)

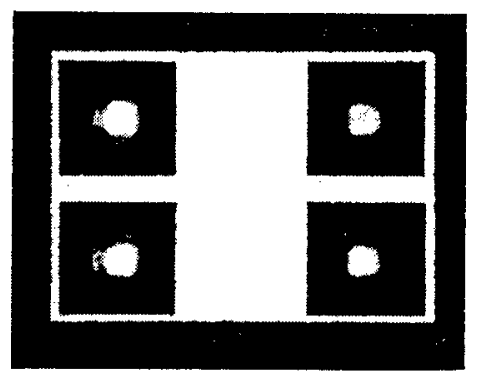

Figure 3.2. Photographs of redesigned pressure sensor, (a) front side, (b) back side.

The redesigned MEMS pressure sensor is roughly the same size as the old sensor (on the Si die level) but has several new or improved features customized for the current need. A new Endevco part number was assigned to this redesigned sensor and it will soon be available overthe-counter as a standard product. The spec sheet for this new part is shown in Figure 3.3. 


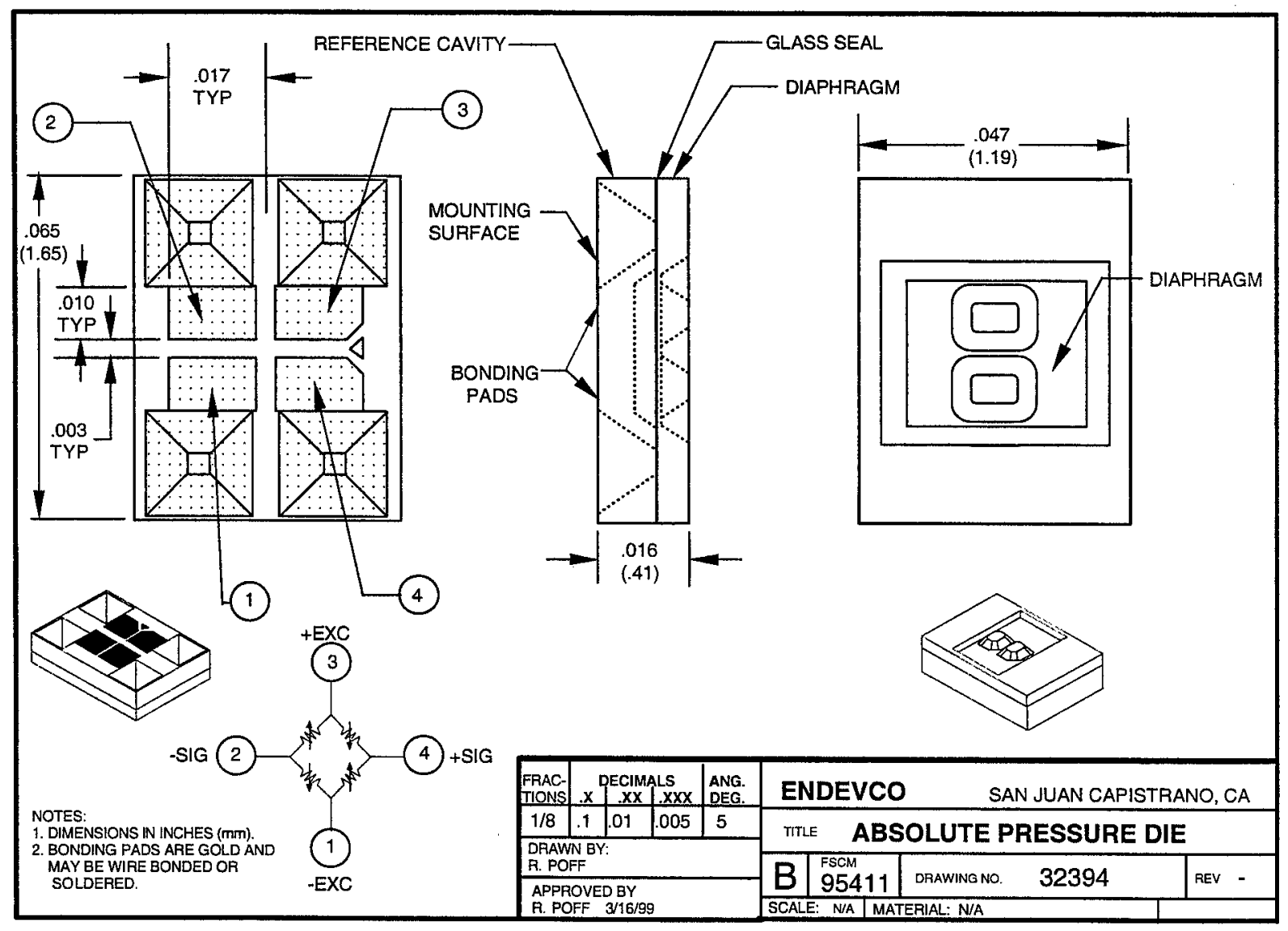

Figure 3.3. Specification sheet for the redesigned sensor.

\subsection{Electronic Circuitry Development}

\subsubsection{TBIM Development}

To achieve the smallest form factor in the pressure belt TBIM, many functions required locally by the sensor, had to be integrated in Application Specific Integrated Circuit (ASIC) format. Since the MEMS sensor is an analog device with an output on the order of millivolts, it is essential for signal integrity to amplify and filter the sensor output and then immediately convert it to a digital signal. Furthermore, since the sensor output is strongly dependent on the ambient temperature, it is also important to correct the pressure reading prior to transmitting to the NCAP. This architecture was necessitated by the IEEE P1451.3 format.

These two distinct functions led to the development of two ASICs, one handling the analog front end with an integral A to D in its output and a digital ASIC which contains a filter, a correction engine, and the bus interface. Prior to the fabrication of the ASICs, the TBIM was built using two circuit boards, one which had all the functions carried out by the analog ASIC and a second board which had the functionality of what was to become the digital ASIC. To mimic some of the limitations inherent in the design of ASICs, such as cell library availability, the digital ASIC board was built based on three Field Programmable Gate Arrays (FPGAs) and a packaged version of the ASIC core processor (8051 equivalent). The three FPGAs functions were: a 
digital filter, the correction engine, and the bus interface. These prototype versions allowed for early testing and troubleshooting the functionality of the design and for software development, prior to the integrated (ASIC) forms. Later, when the ASICs became available, packaged forms of the digital and analog ASICs were used on a circuit board together with a number of passive components to mimic the functionality that would be present on the MCM. This provided a way to debug the MCM level functions on a circuit board level configuration, where we could probe directly different nodes and use cuts and jumpers, if necessary. The TBIM block diagram is shown in Figure 3.4 and a photograph of the discrete version is shown in Figure 3.5. The discrete version of the TBIM, which utilized an analog and a digital board, was used in the laboratory for testing. An early version of the prototype TBIM MCM is shown next to it to indicate the overall desired shrinkage factor.

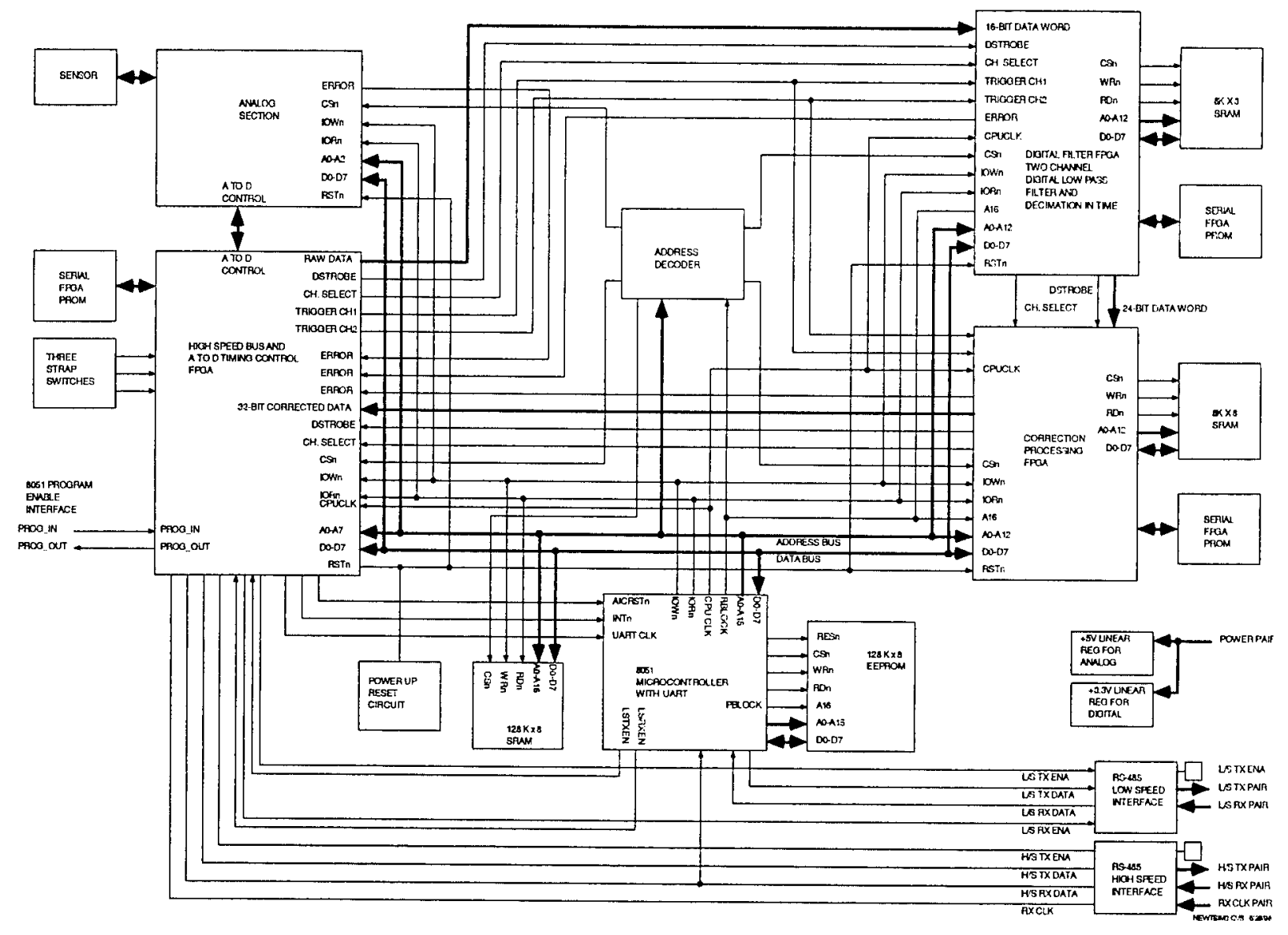

Figure 3.4. TBIM block diagram. 


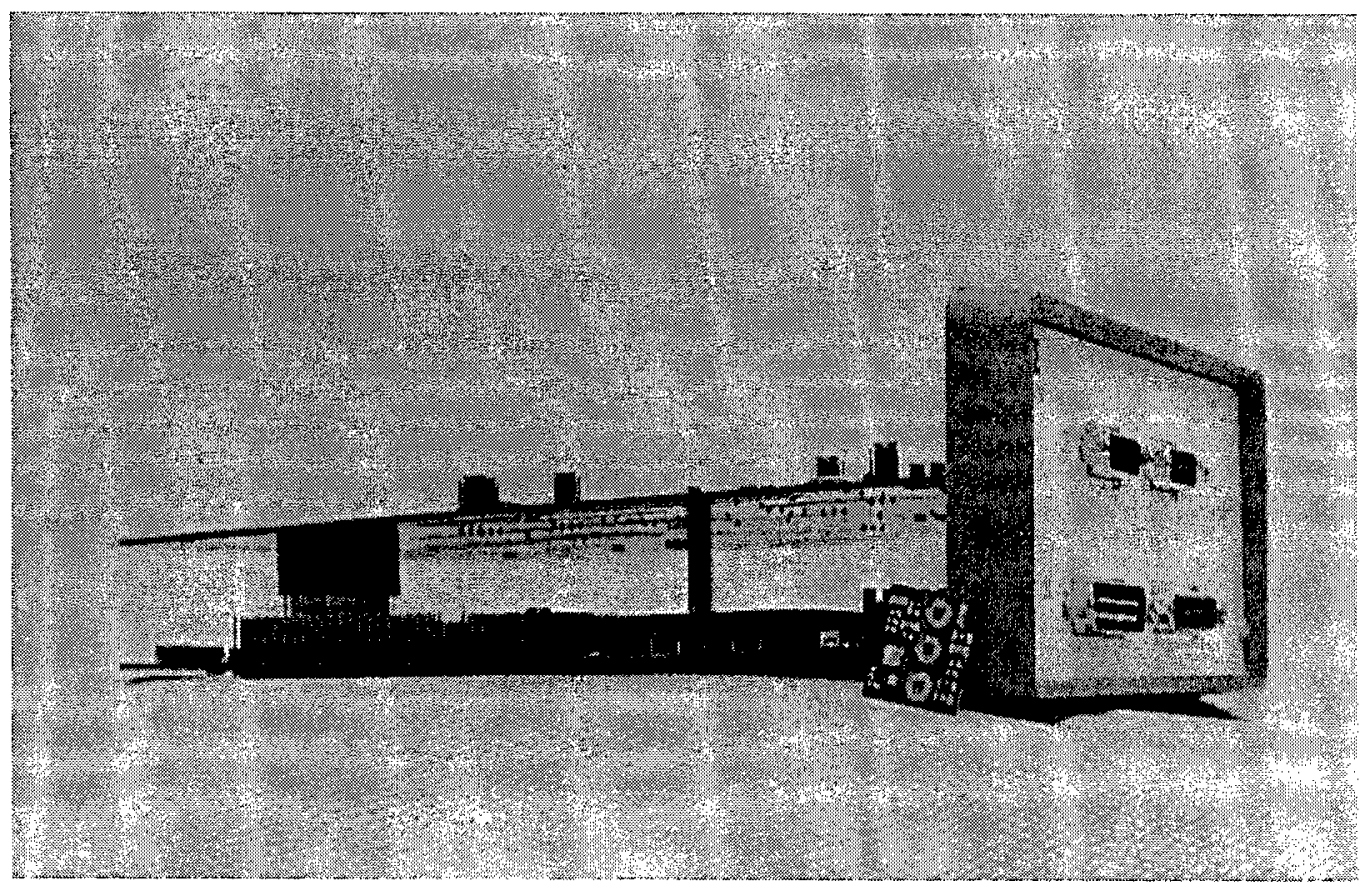

Figure 3.5. The discrete version of the TBIM.

Several units of the discrete TBIM were built and tested in the laboratory, both as isolated units and also strung together to mimic the overall pressure belt function. Within this box, an analog board mimicked the functions of the analog ASIC. The analog ASIC provides the flexibility to condition various transducer types, resistive (full bridge, half bridge, quarter bridge), piezoelectric, capacitive, voltage, and current. Simply by interconnecting the analog ASIC in various ways to external discrete components located on the MCM, one can change its function and use it to measure, for instance, acceleration or strain. Each configuration will require a different MCM layout but the same analog ASIC can be used. To test this capability in the analog board, jumpers were installed in the prototype PCB to mimic the many MCM possibilities (we did not want to create various PCB prototypes for each possible configuration). The analog ASIC functional block diagram is shown in Figure 3.6. Figure 3.7 shows pictures of the PWB and ASIC die as well as the shrinkage between the two. The foundry for the ASIC die was AMS. Some of the analog ASIC functions are listed below:

- Programmable Gain

- Instrumentation Amplifier (IA)

- Binary Programmable Gain Non-Inverting Amplifier

- Setting Gain

- Programmable DC Offset Adjustment

- Programmable Current Excitation

- Self-Test

- Analog Anti-Aliasing Filter 


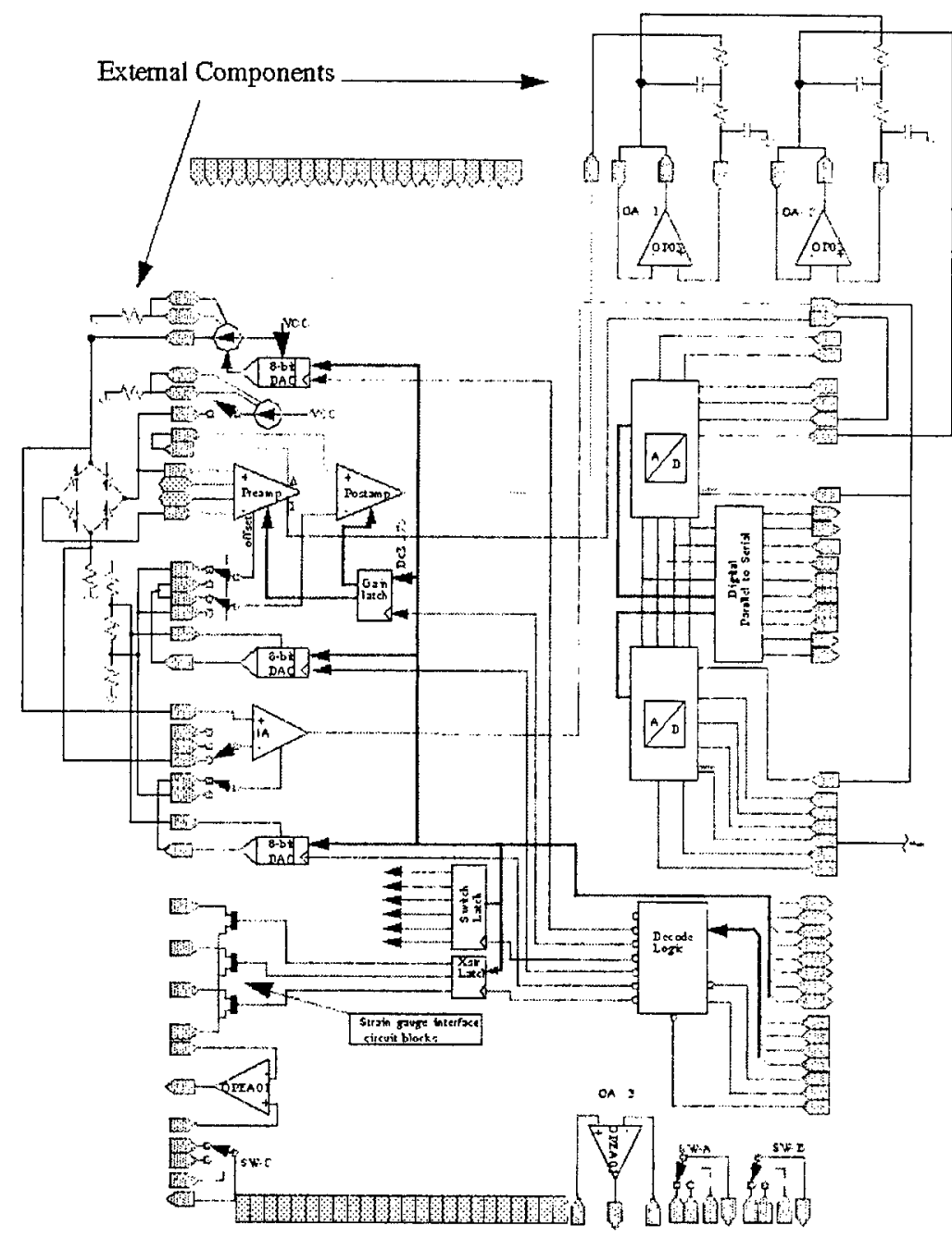

Figure 3.6. Analog ASIC Block Diagram.
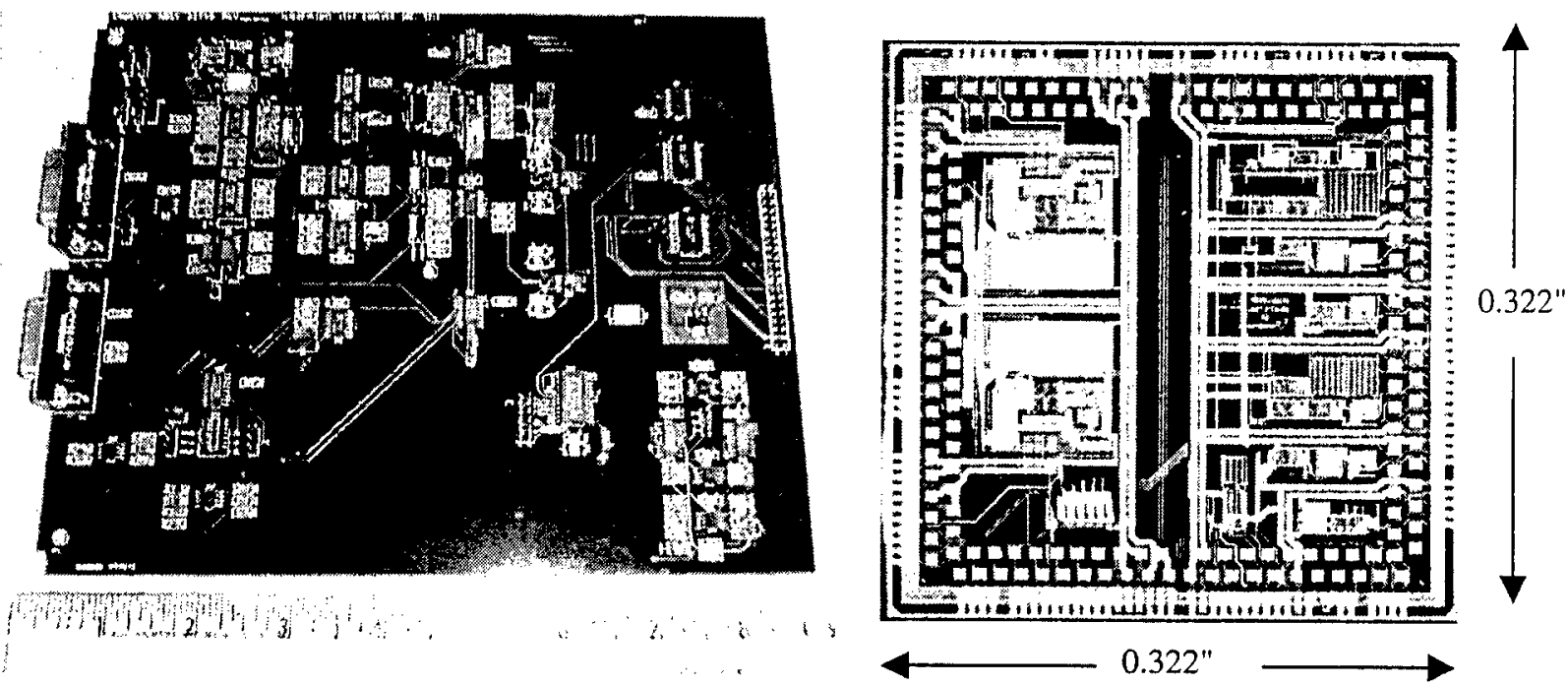

Figure 3.7. Prototype PWB version of the analog ASIC and its die form. 
A parallel path of development was taken on the digital ASIC. The digital ASIC functions consist of an 8051 microcontroller, a programmable digital filter engine, a multinomial correction engine and a high-speed transmitter/receiver interface. The two engines and the highspeed interface are realized in three different FPGAs. The high-speed bus interface FPGA allows the TBIM to communicate with the TBC (Transducer to Bus Controller) over a digital transducer bus and allows synchronization and control over the local ADC sampling process.

The receiver circuit performs hardware address filtering for all packets and hardware command decoding for specific commands. This allows deterministic time response to specific commands such as the Trigger command. The receiver also allows messages, which are not decoded by the hardware, to be queued for interpretation by the associated microcontroller. The SAR ADC interface circuit synchronizes and controls the $\mathrm{A}$ to $\mathrm{D}$ sampling process and the transfer of data from the ADC to the digital filter. The Sigma Delta ADC interface circuit synchronizes and controls the optional Sigma Delta ADC. The AIC51 bootloader circuit enables the AIC51 microcontroller to be placed in a special bootloader mode, which allows reprogramming of EEPROM containing the AIC51 firmware. The digital filter interface circuit allows the raw ADC data to be written to the digital filter. The microcontroller interface allows the AIC51 microcontroller to control the operation of the bus interface and sampling process. The transmitter circuit performs transmission of data onto the transducer bus. This includes transmissions initiated by hardware decoded commands such a Read Transducer Data command, or transmissions initiated by the microcontroller such as a response to a Status Request command. The transmitter also performs transmission of the UUID sequence allowing each TBIM to be uniquely identified by the TBC. A drawing of the digital ASIC functional block diagram is given in Figure 3.8 and photographs are shown in Figure 3.9. The foundry for the digital ASIC was AMI.

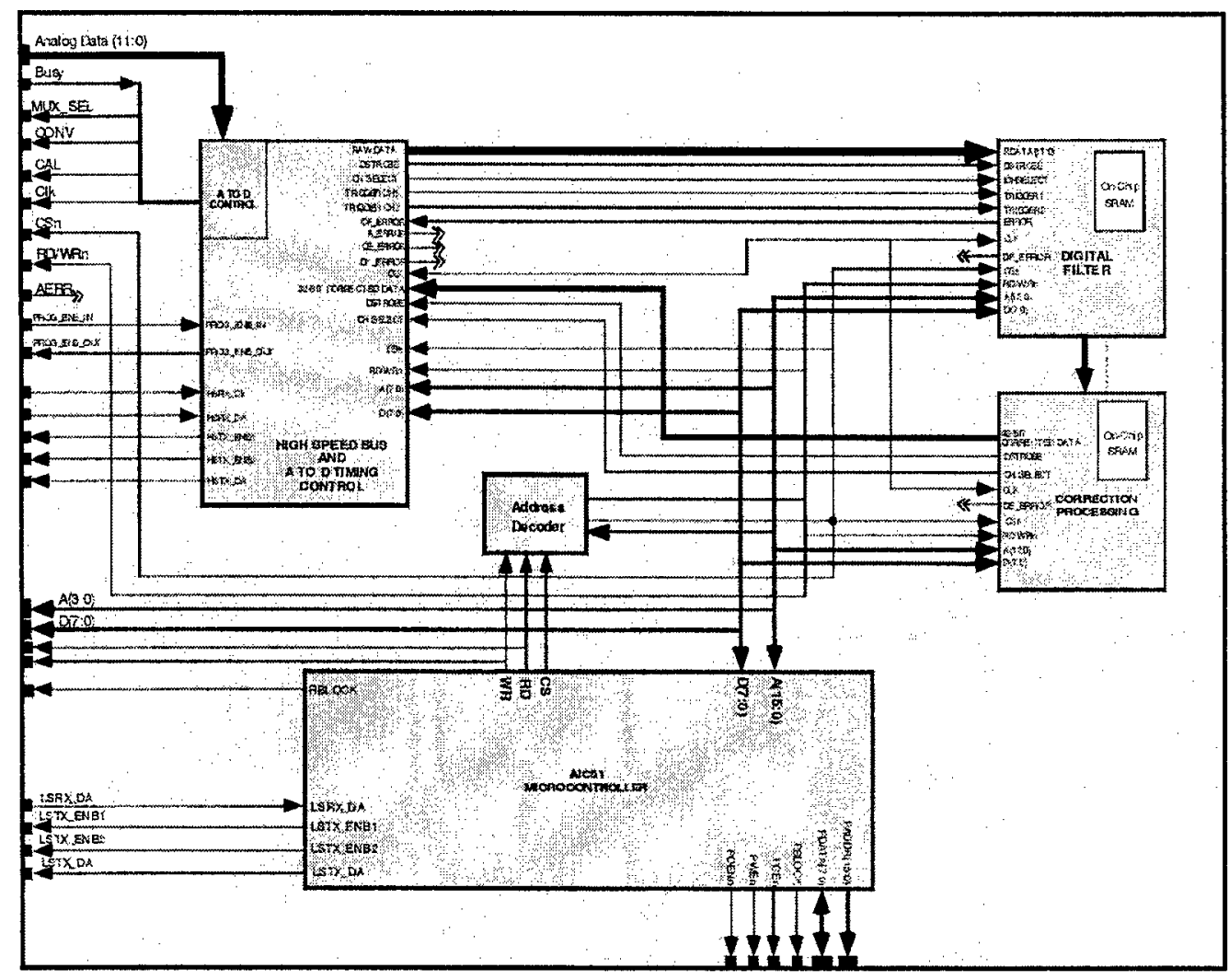

Figure 3.8. Block diagram of the digital ASIC. 

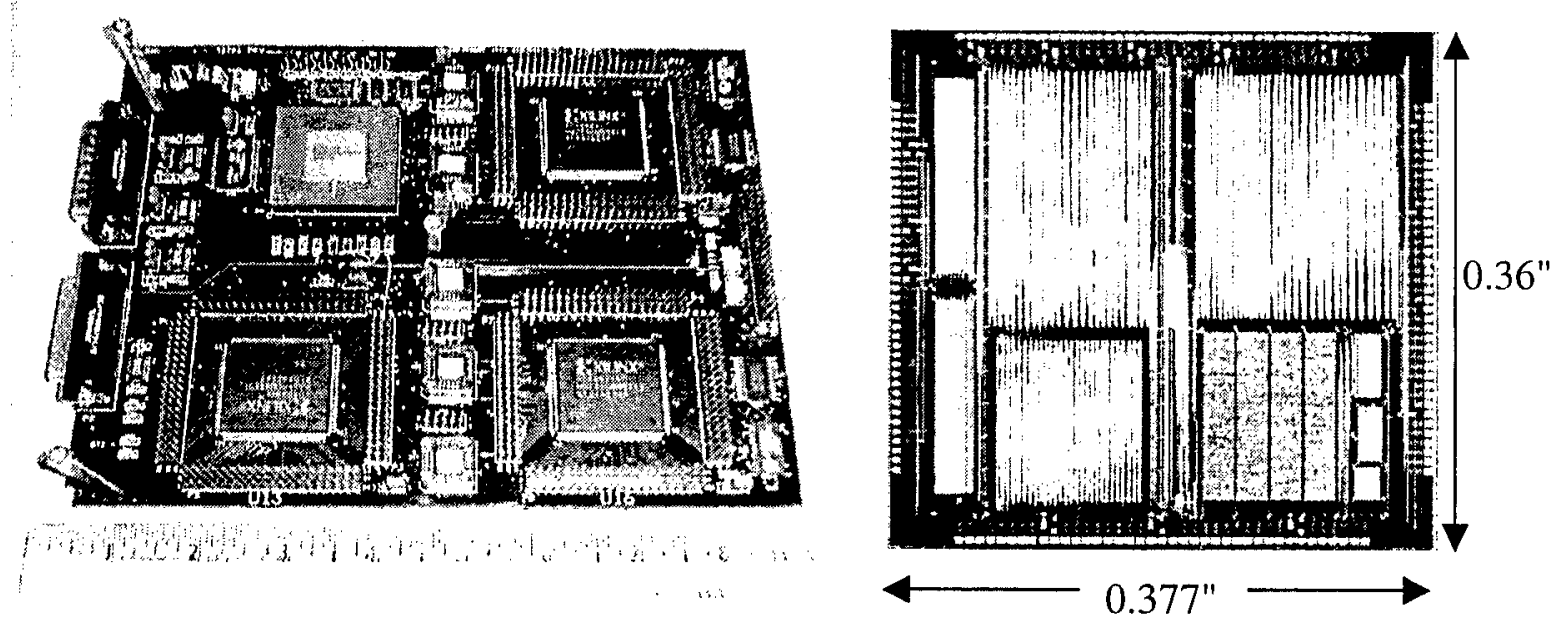

Figure 3.9. Photographs of the digital board and the digital ASIC.

Finally, several TBIM boards were constructed using PWB technology, which included the ASICs in a packaged format. This allowed for verification of the full functionality of the individual TBIM as well as a number of TBIMs hooked together before we went to the MCM version. The reason for this caution was the fact that in an MCM one does not necessarily have $100 \%$ nodal visibility, so if there is a problem it becomes hard to troubleshoot. This board with the packaged ASICs is shown in Figure 3.10.

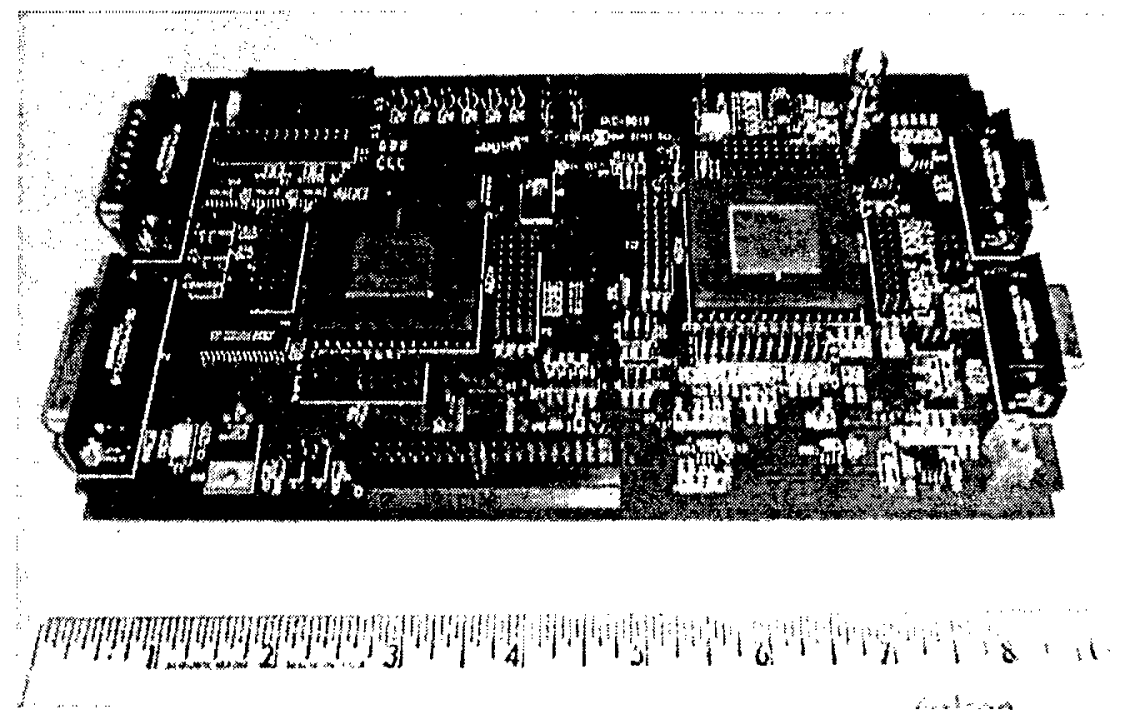

Figure 3.10. A photograph of the discrete version of the TBIM using the packaged ASIC die.

\subsubsection{Lab Test and Calibration}

A calibration of the prototype TBIM integrated with a MEMS pressure sensor was performed using an analog board (modified to correct some of the non-linear performance). The calibration incorporated all the functional elements of the ASIC designs along with the design intended for the pressure belt segment. We used automatic test equipment and a Labview $®$ application to control the test program. The test was designed to perform 21-point calibrations over 10 temperature settings from 0.5 to $15 \mathrm{psia}$ and temperatures from -50 to $50^{\circ} \mathrm{C}$. A surface fit was calculated using the 190 data points collected from the test. This fit represented the type of 
polynomial which is used in the correction engine of the digital ASIC, having the capability to provide output in engineering units. What we discovered was that residuals from this surface fit do not exceed $0.04 \%$ of the 15 psia range. This is shown in Figures 3.11 and 3.12 for the measured pressure and temperature, respectively. This means that, at least in the lab, the system meets the desired specification of equal to or better than $0.1 \%$ accuracy in the 15 psia range, using electronic correction. Figure 3.11 shows the residuals (departure of the data from a fifth order polynomial fit) of the laboratory pressure measurements (corrected for deviations) vs. a standard pressure measurement, as a function of temperature. Since the sensor data accuracy varies with the absolute pressure and temperature, both have to be measured and a correction algorithm is used to obtain the desired values. This correction engine is resident in the digital ASIC. The fit is empirical, but as one can see the departure from the actual pressure is very small, within $0.1 \%$ of the full scale value, thus validating the use of the correction engine. The electronics used for this measurement was the discrete version of the prototype belt, which has the full functionality of the final ASICs implemented in FPGA format. Figure 3.12 shows residuals of the laboratory temperature measurements (the temperature channel output was corrected for deviations using the algorithm in the digital ASIC) vs. a standard temperature measurement. The surface fit was calculated using the same fifth order polynomial as in Figure 3.11. Resulting residuals are within $\pm 1.5^{\circ} \mathrm{C}$. This was done to check the temperature measurement accuracy using the MEMS sensor and the correction engine in the TBIM.

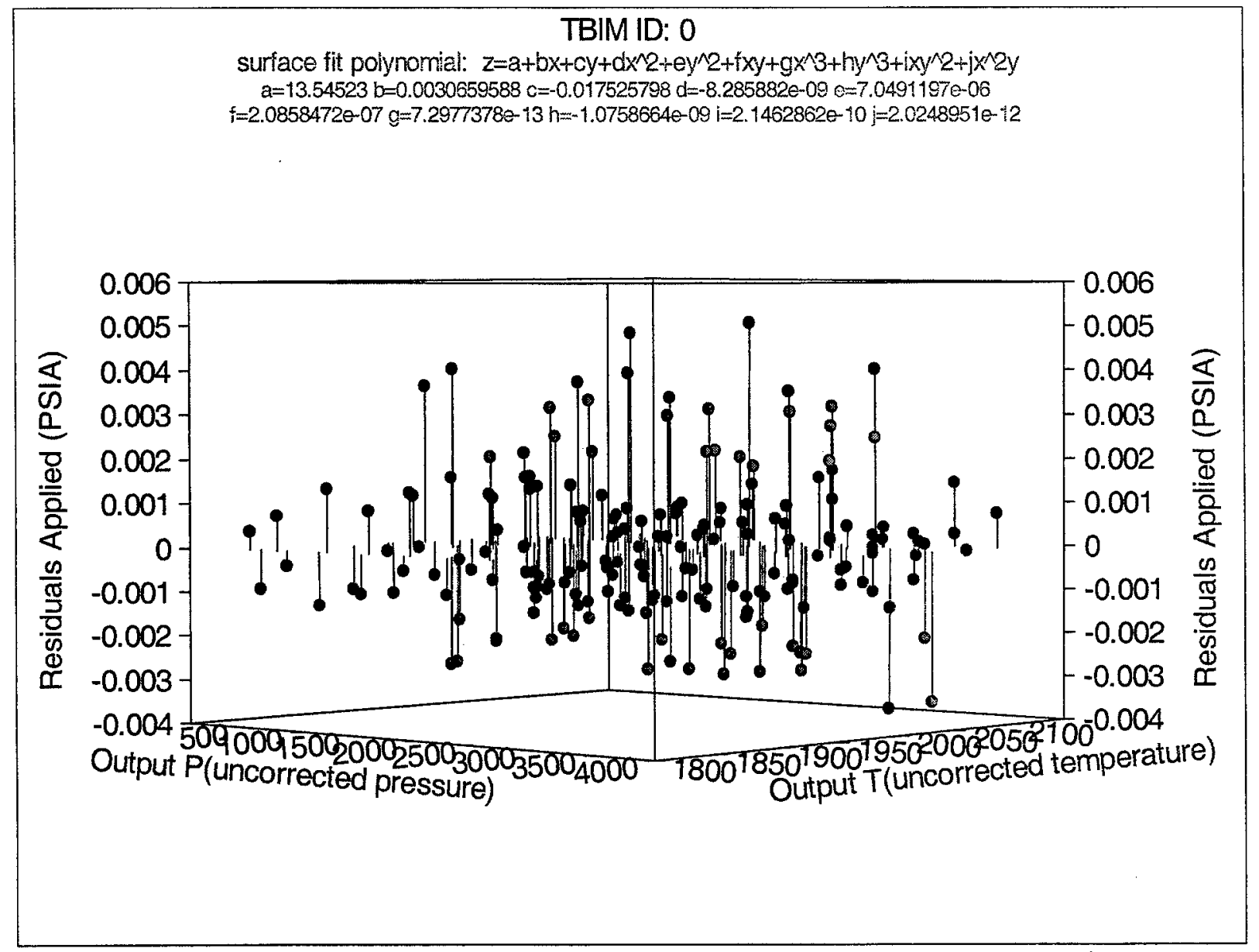

Figure 3.11. Surface fit of the pressure channel output vs. a standard, as a function of temperature using the TBIM electronics 


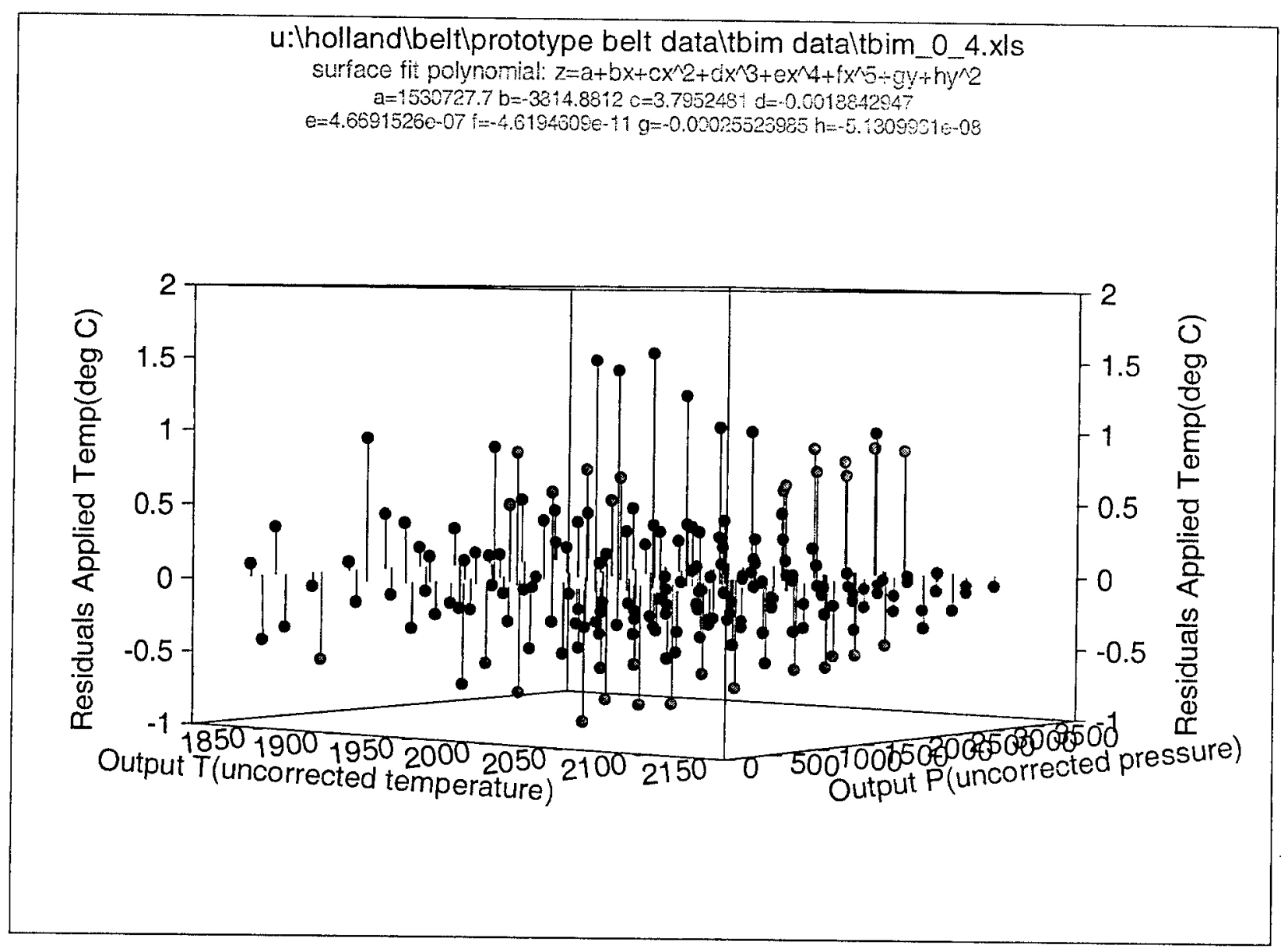

Figure 3.12. Surface fit of the temperature channel output vs. a standard using the TBIM electronics. 


\subsection{PRESSURE BELT TRANSDUCER BUS CONTROLLER}

\subsection{TBC Hardware Architecture}

Figure 4.1 depicts schematically the functional relationship between the TBIMs and the TBC.

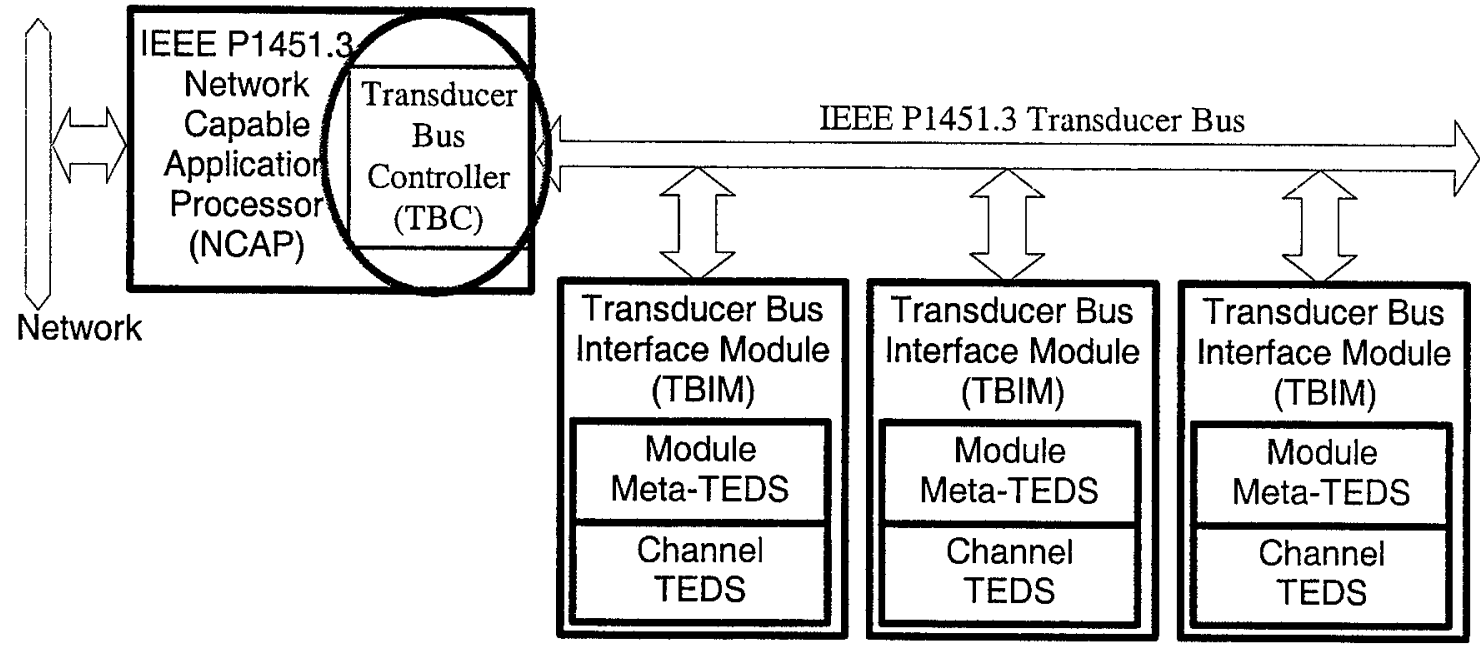

Figure 4.1. The architectural relationship between the NCAP, the TBC and the TBIMs.

Figure 4.1 shows the TBC connected via an IEEE P1451.3 transducer bus to multiple TBIMs. The TBC is part of the Network Capable Application Processor (NCAP). The NCAP can then be connected to a communication network used to interface to a host computer. The TBC can also be configured as a host computer interface directly (no NCAP) without the communication network. For testing the prototype pressure belt, and the TBIMs contained within it, the TBC was configured as part of an NCAP. A schematic is shown in Figure 4.2. This prototype NCAP has been configured using a set of PC104 form factor circuit boards, consisting of the power module, flash disk, 586 PC, Ethernet controller, and TBC. Figure 4.3 shows a photo of the current NCAP implementation. Although there are plans in the future to make the NCAP smaller with a more powerful processor, it is not intended to be placed on the airplane wing. It would most likely be inside the airplane cabin.

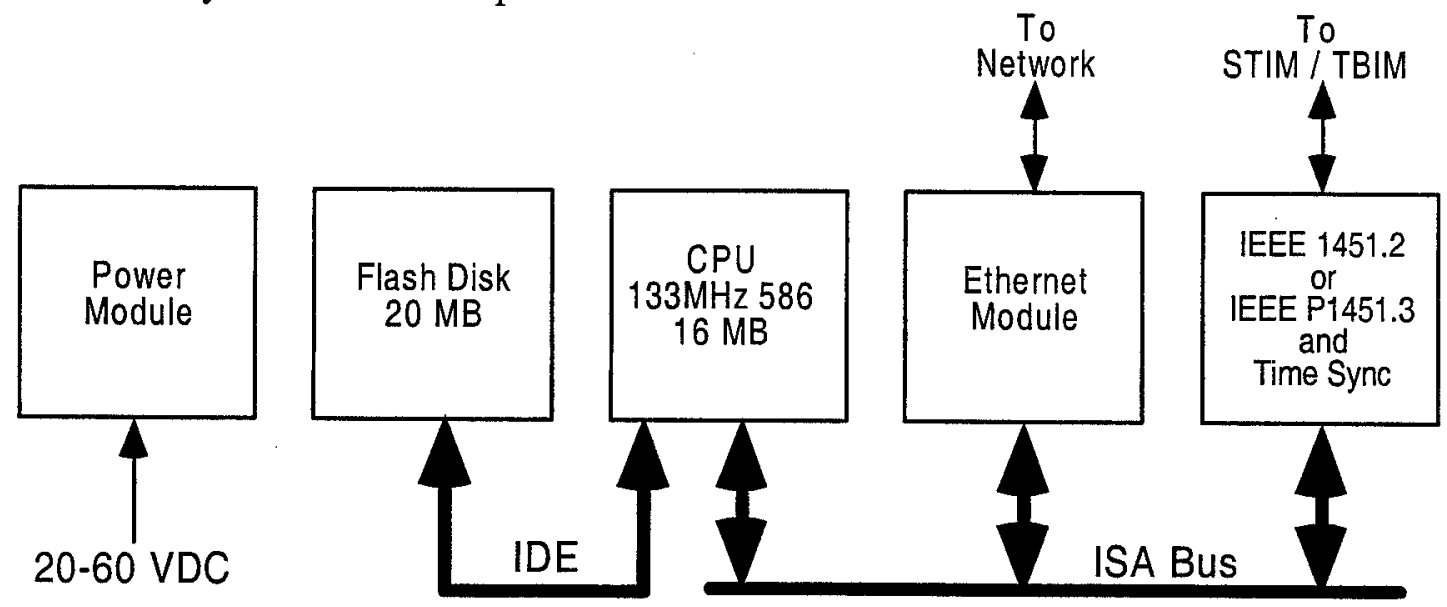

Figure 4.2. Current configuration of the TBC. 


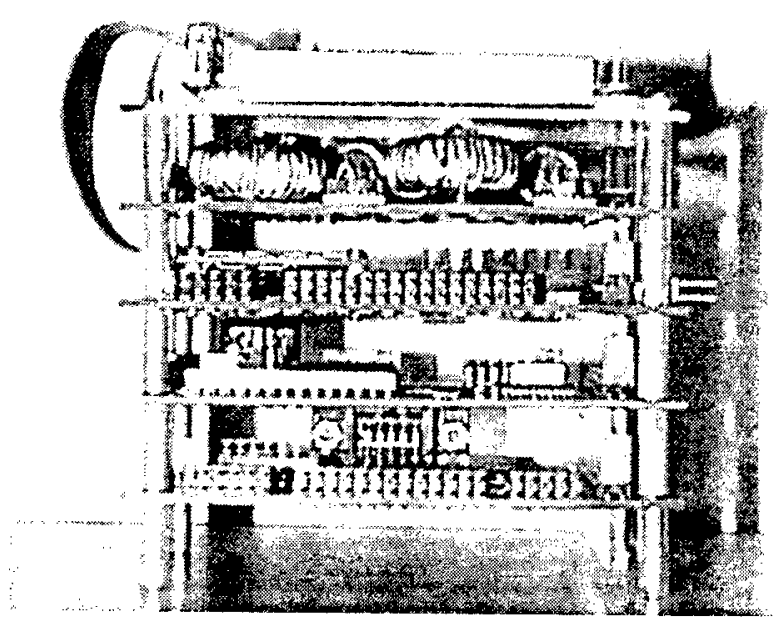

Figure 4.3. Current implementation of the NCAP, which will be used for Flight Testing.

\subsection{TBC Software Architecture}

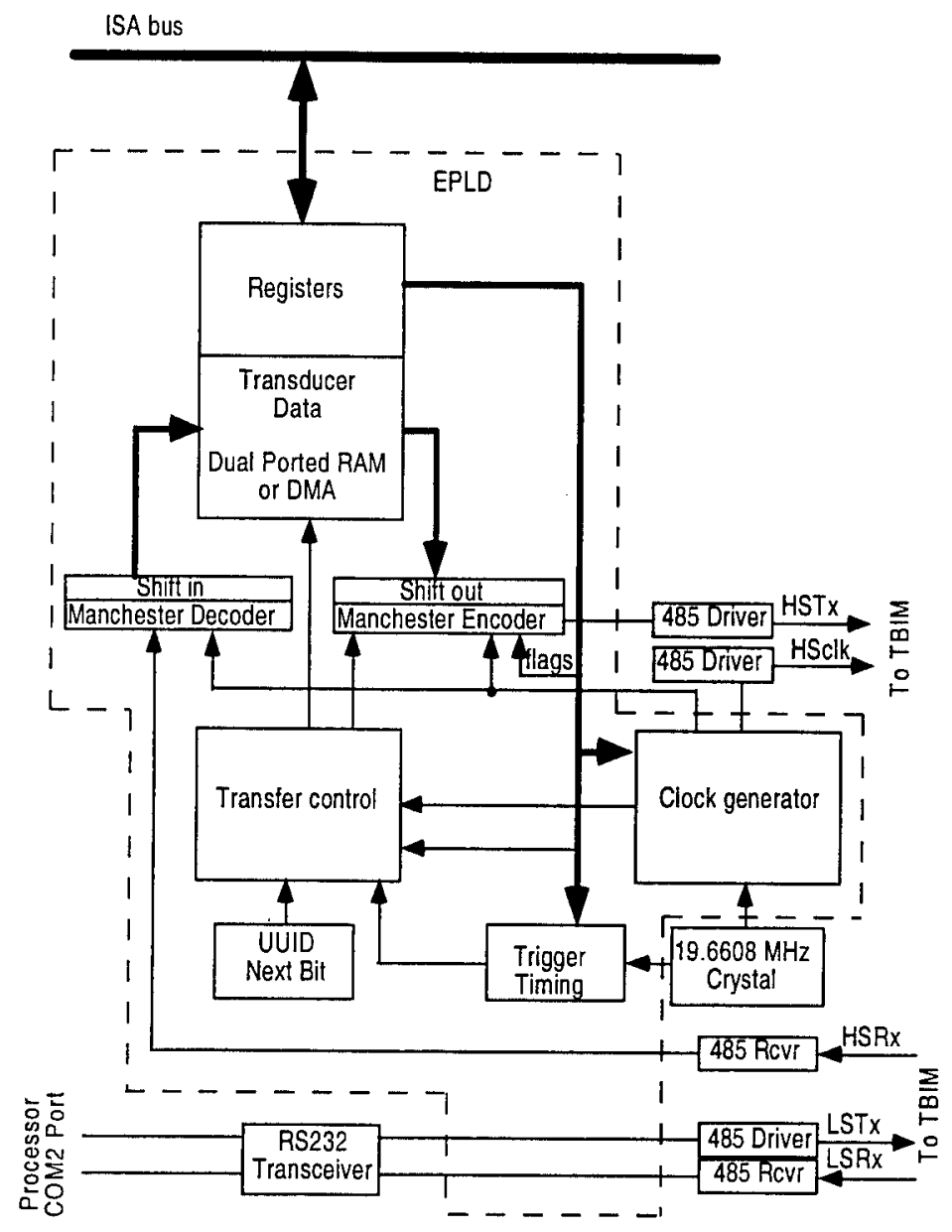

The TBC software uses the Linux operating system running on the NCAP PC104 card stack. Software developed for the pressure belt project includes a custom device driver for the high-speed bus interface, a set of library functions for using the device driver, and several user programs. The existing Linux serial port device driver is used for access to the low speed bus. The programs are exercised via a telnet connection from a remote Win95/NT PC host computer. Permanent storage for data and script files is provided via shared folders on the remote host. In addition to meeting the protocol requirements defined in the Pressure Belt ICD, the software also provides for "breaking" the protocol in order to fully test the interface. A diagram of the TBC functionality is shown in Figure 4.4, while a description of the TBC Software Architecture is shown in Figure 4.5.

Figure 4.4. Diagram showing the electronic functionality of the TBC. 


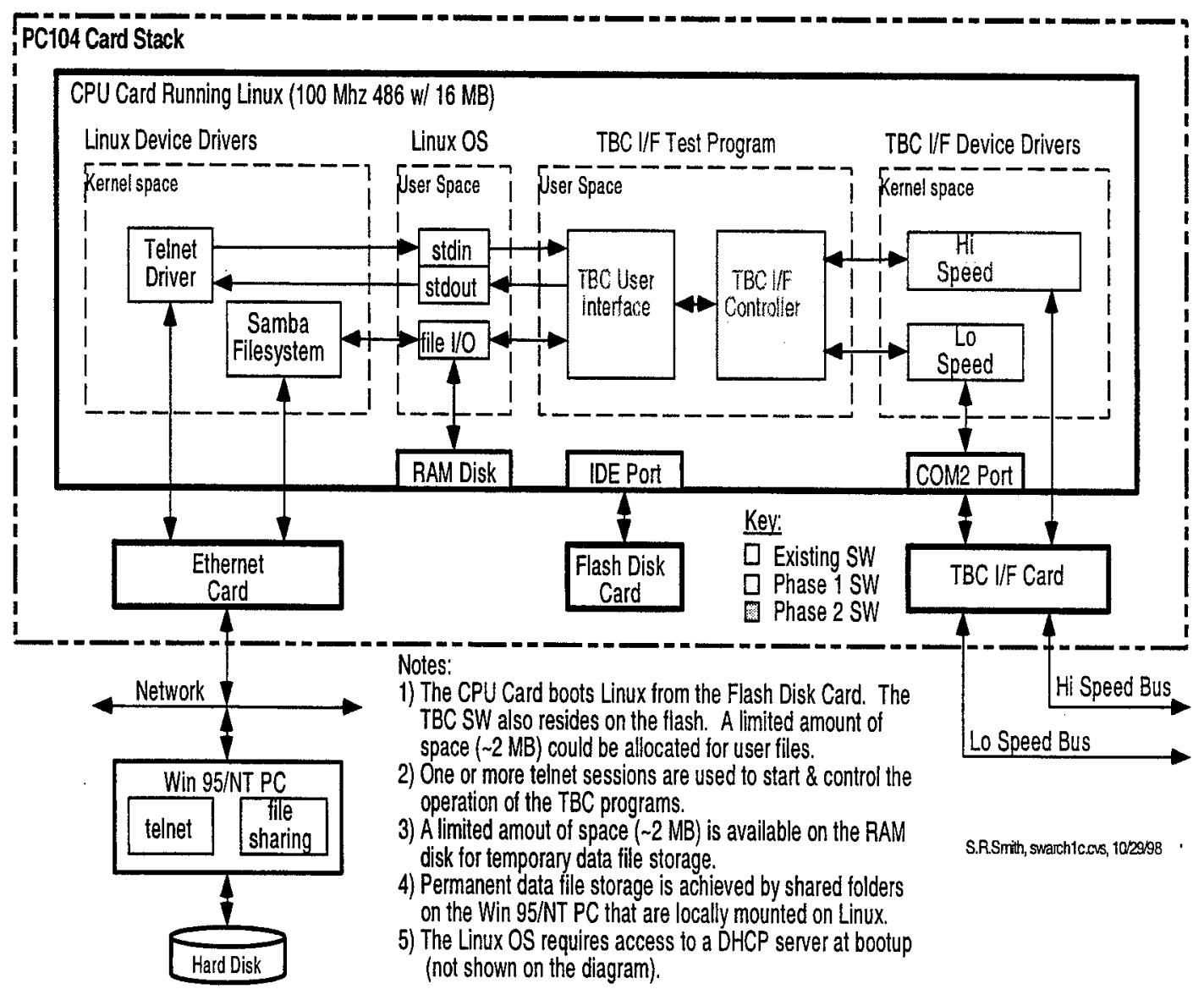

Figure 4.5. A block diagram of the TBC software architecture.

The TBC custom high-speed device driver provides access to the TBC high-speed bus interface hardware. The TBC driver program is targeted to the TBC HW/SW developers and provides low-level access to the device driver for debugging purposes (e.g. HW I/O peek \& poke, HW RAM read \& write).

The TBC downloader program is targeted to support initial checkout of the TBC $\Leftrightarrow$ TBIM interface and provides TBIM memory download \& upload access via the low speed bus.

The TBC control program is targeted to support initial checkout of the TBC $<=>$ TBIM interface and provides mid-level, integrated access to the high speed \& low speed buses. It may be used to issue any sequence of command bytes to the TBIM. The TBC program is targeted to support end user testing with the pressure belt and provides high-level, integrated access to the high speed \& low speed buses. Persistence of the pressure belt node configuration is provided via database operations. The program ensures consistency between the database configuration and the actual bus setup before allowing any commands to be issued on the bus. All of the TBC programs use a command-line based interactive design that allows the user to enter commands to be carried out by the software. The commands can also be saved in an ASCII script file for batch processing. 


\subsection{PACKAGING TECHNOLOGY DEVELOPMENT}

The role of packaging is to pull all the parts together so that the final product is optimized in terms of size, utility, and cost. In this program the major drivers were:

a. The total thickness of the pressure belt. Including all the layers, adhesives, and devices the requirement was that the pressure belt should not exceed 0.1 " in height from the surface of the airplane. The reason had to do with the fact that a thicker belt could disturb the boundary layer, and thus the measurement accuracy. Considering the thickness of adhesive used to tack the pressure belt to the airplane skin surface is 0.022 ", the thickness available for the pressure belt was less than $0.08 "$. This requirement led to the use of unpackaged devices on the TBIM surface. Since Boeing has a large experience base in fabricating silicon-on-silicon multichip packages, it was natural to choose this technology to fabricate the TBIM circuitry. To provide protection of the devices against environmental factors, we had to use thin protective coatings, which provided reliability without hermeticity.

b. The objective to achieve $0.1 \%$ accuracy over the whole temperature range of operation. The choice of a silicon substrate MCM provided a number of advantages in this situation. First, it was a perfect match in the differential thermal expansion between the MEMS device and its substrate. This was also true for the ICs. The reason stress and temperature are of importance is because the MEMS sensor is essentially a temperature sensitive stress gauge, so any changes in stress or temperature at the sensor can be misinterpreted as false pressure readings. Second, a stiff substrate such as silicon provided a stress redistribution layer. This is especially important for a surface that generates very high levels of strain (airplane wing). This was further improved by adding an adhesive that bonded the silicon MCM to the belt segment such that the stress coupling between the two was minimized. Third, since single crystal silicon is a very good thermal conductor, the silicon MCM also acted as a thermal redistribution layer. Another important element was the use of the MEMS device without its conventional package. The reason was to enhance its sensitivity as much as possible, even though this created some limitations on the robustness of the device configuration.

c. Ease of handling and versatility. This was addressed by making the belt in segments using a flex board-type substrate, which can be connected to each other, as needed. Also, a fairing was used to protect the pressure belt from human error and mishandling as well as to provide an aerodynamic surface.

In the following Sections, we will discuss all these issues in detail and will provide the various approaches and solutions we arrived at, as a result of testing done in a realistic environment (flight). Although our packaging approach addressed the requirements set forth, it also created some unique challenges. Unpackaged electronic devices have been used in the past for a number of applications, using Chip-On-Board technology (COB). COB uses unpackaged dice on a circuit board, either rigid or flex. The devices are electrically interconnected either using tape automatic bonding (TAB) or wire-bonding. The bare devices are protected by a glob-top epoxy coating, which is similar to plastic encapsulated die. Boeing already had some experience in this area. However, this technology has been typically used to cut costs and reduce weight for use in benign environments, without any significant emphasis on reliability. Our challenge was to apply this technology to a rather hostile environment (flight test) and to ascertain that it was reliable for extended usage. Testing of the various packaging configurations was done both in 
the laboratory and in the field with excellent results. Figure 5.1 illustrates the various parts of the pressure belt.

The schematic drawing shown in Figure 5.1 (a) is a section of the pressure belt with the $\mathrm{Si}$ MCMs (TBIMs) on it, the segment wiring, the inter-segment connection, the registration marks, and a number of devices resident on the segment itself. Figure 5.1 (b) is a cross-sectional view with the vertical scale enlarged in order to show the various layers. One can see the TBIM with its connection to the flex tape carrier, the role of the protective coatings, the packaging of the MEMS device and the ICs, and finally the fairing. In the following Sections, we discuss the various features. Additional information regarding the packaging technology development is given in references 5-8 in Appendix A.

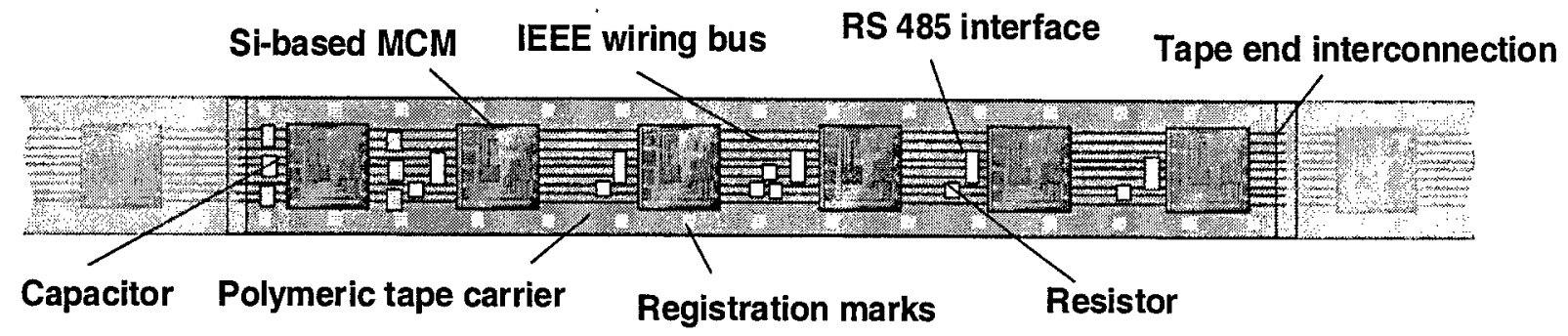

(a)

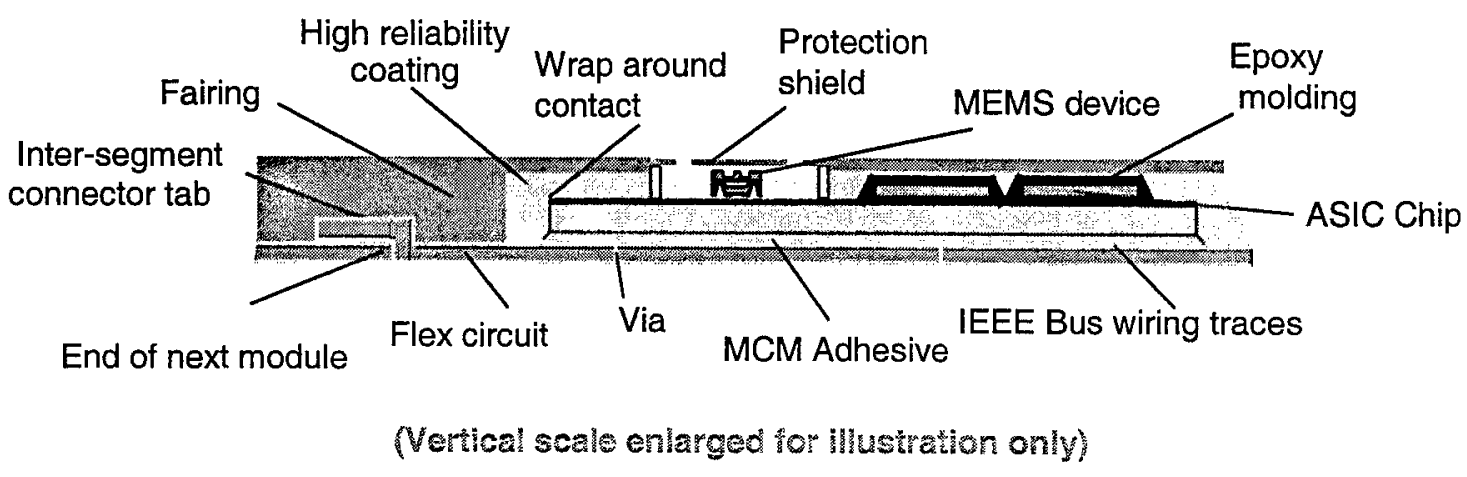

(b)

Figure 5.1. Schematic: (a) Drawing (top view) of the production pressure belt showing the various parts, (b) Representation of the pressure belt cross-section.

\subsection{MEMS Device Packaging}

The MEMS device is being used in its bare silicon form, in a flip-chip configuration, similar to an IC die. However, as opposed to an IC, one cannot apply any protective coatings on its surface because that would change the characteristics of the sensing membrane. A short glass tube encircles the sensor acting as a dam, to isolate the sensor from the protective overcoat layer applied to the rest of the module. Inside the glass seal there is a low-stress silicone gel (DC Q14939), which seals the pad area around the sensor feedthroughs against moisture incursion. This silicone was chosen because of its low modulus of elasticity, which would not create any undue stress on the sensor. The sensor bonding to the gold pads on the multichip module (MCM) is 
done either through conductive epoxy or Sn/Au reflow. The sensor is attached directly to the silicon substrate of the MCM, through an etched via in the copper/polyimide interconnect layers in which the interconnections on the MCM reside. Having the MEMS device on the interconnect layer would negate the advantages of having the MEMS attached to a silicon substrate, since the interconnect dielectric and the copper lines have much higher expansion coefficients than silicon.

In our initial design, there was no protection over the sensor membrane area. In fact, the first flight test of this MEMS sensor on a 757 aircraft was done with the sensor totally exposed. However, such protection became necessary after we experienced failures in the field during one of the flight tests on a 737-series aircraft designated by The Boeing Company as the Boeing Business Jet (BBJ). Figure 5.2 shows the sensor module installed on the BBJ forward slat, along with its fairing (white). The fairing provides an aerodynamically smooth surface to prevent boundary layer separation, which could disturb the measurement we are trying to make. As one can see, there is a hole right above the sensor location, which makes the sensor susceptible to direct impact damage. This type of damage was observed when the BBJ finished its series of flight tests and was returning from Mesa, AZ to Seattle. During its final approach to Boeing Field, at about 5,000 ft and an airspeed of 250 knots, both operational MEMS devices on the slat ceased to function within about two seconds of each other, shown in Figure 5.3. As one can also see from the data in Figure 5.3, the moment the failure occurred the airplane encountered severe turbulence as evident from the acceleration spectrum inside the cabin. After checking the pilot's $\log$, we found that the cause was a severe rainstorm the aircraft encountered over the Seattle area. Considering the severity of the acceleration spectrum inside the cabin, it is probably a safe assumption that this was much more severe on the airplane wing.

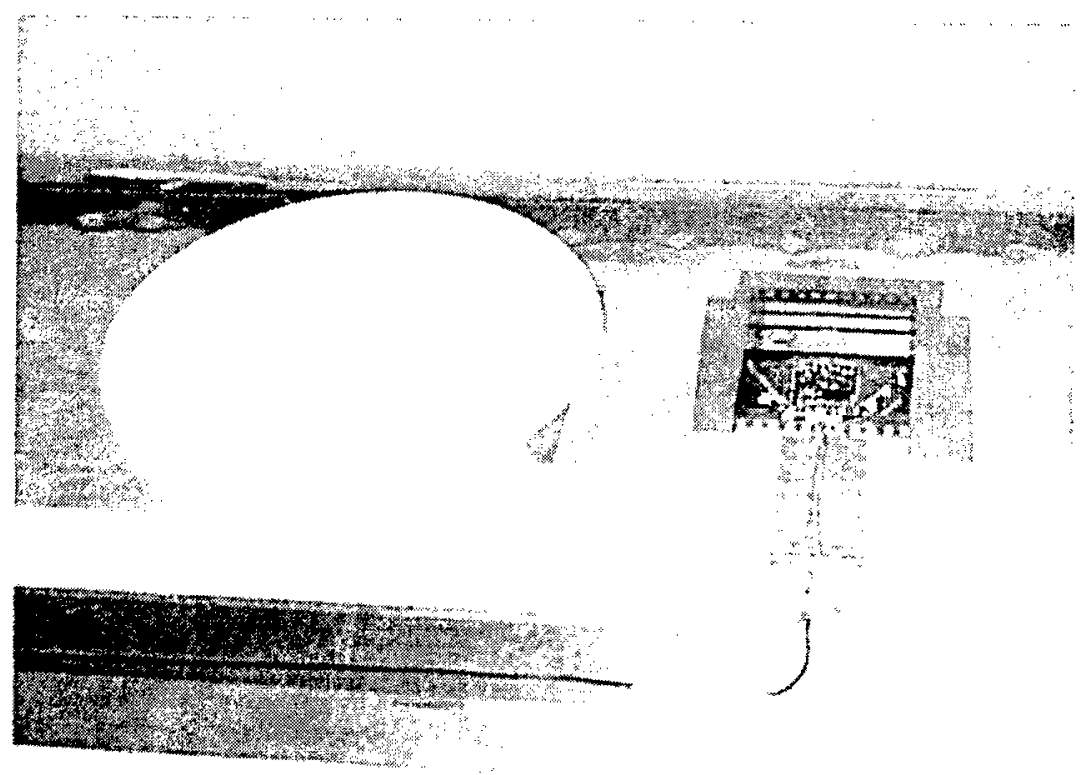

Figure 5.2. The TBIM installed on the BBJ for flight testing. 


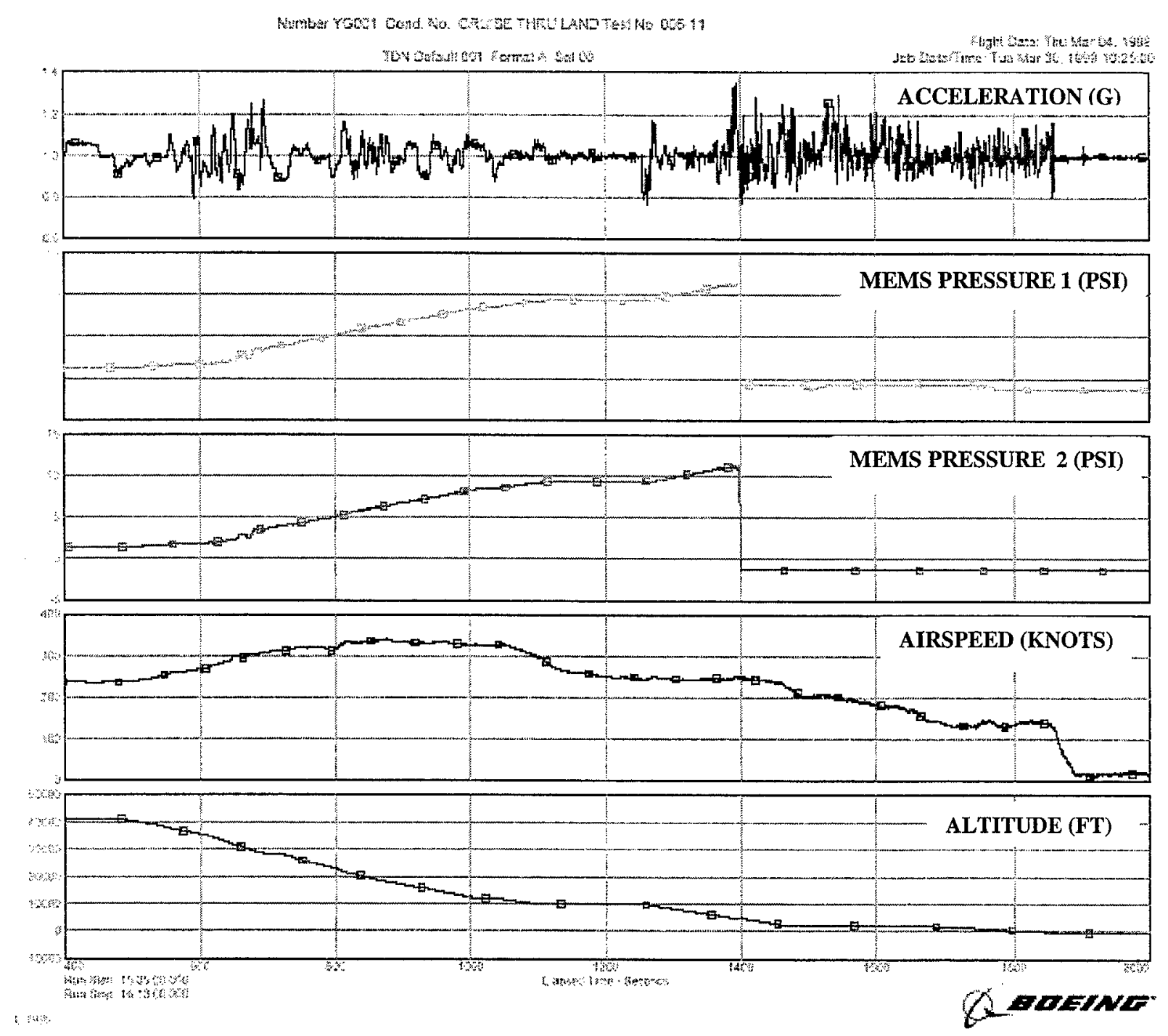

Figure 5.3. Flight test data showing the failure of the MEMS sensors.

To ascertain the type of damage that had occurred, the module on which the MEMS device resided and all the interconnect wiring were examined. No significant damage was noticed, which narrowed the cause of failure down to the MEMS device itself and its interconnection to the MCM substrate. After we made certain that the bonding to the substrate was still good, a scanning electron microscopic examination of the failed MEMS device was performed. It revealed that there was a crack in the sensor diaphragm, which could have been caused by direct impact from the raindrops, while the slats were deployed for landing. A picture of the cracked diaphragm area is shown in Figure 5.4 (a) - (c), in successive magnifications. The fracture is clearly evident in (c) but can be barely discerned in the lower central region of (b). The fact that no other significant damage had taken place either in the electronics or the module packaging was verified by reworking one of the modules, where the MEMS device was removed and replaced with a functional one. After replacement, the TBIM functions were verified to be within its operational envelope. 

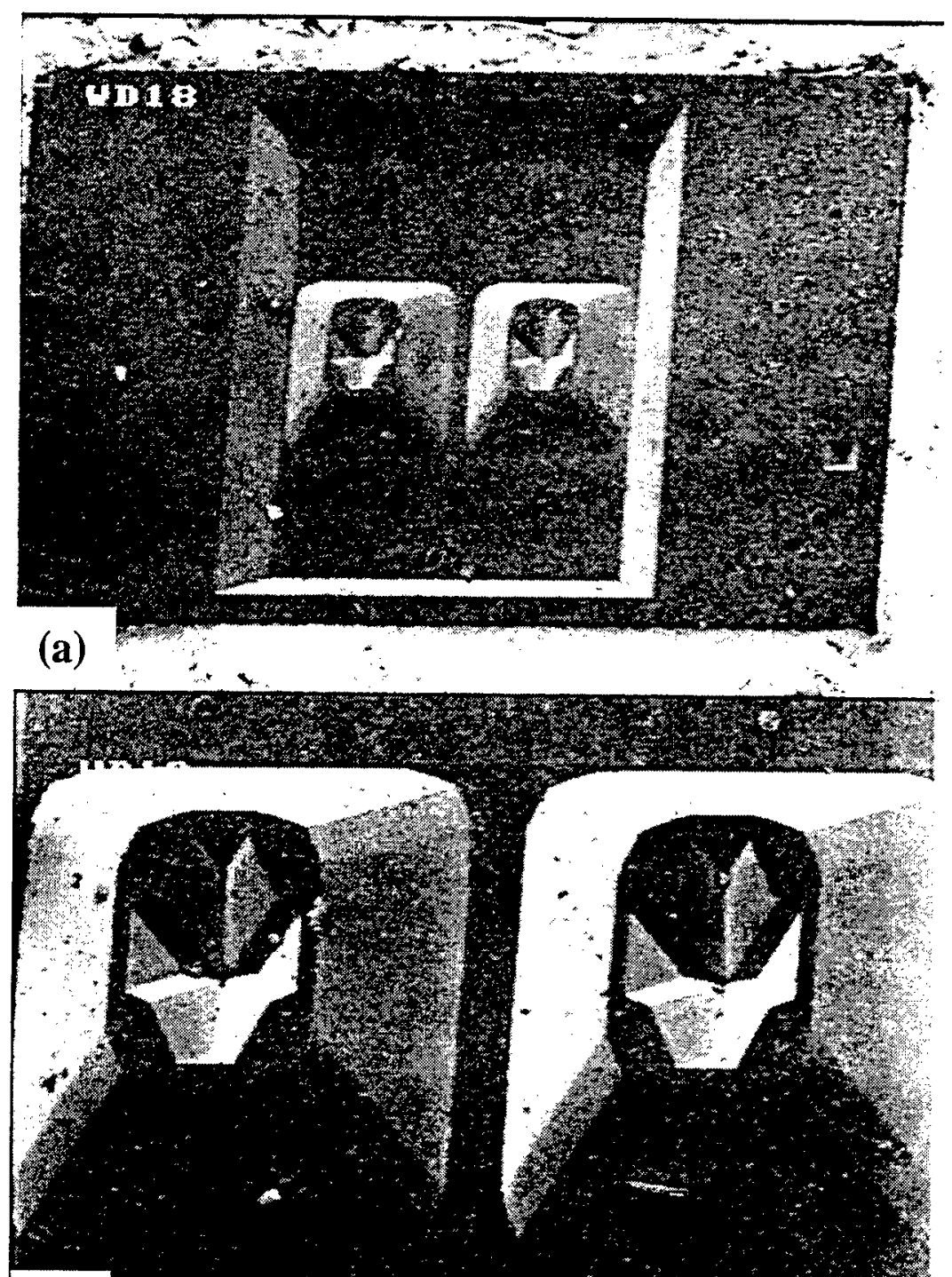

Figure 5.4. SEM

Micrographs of the fractured diaphragm in different magnifications.
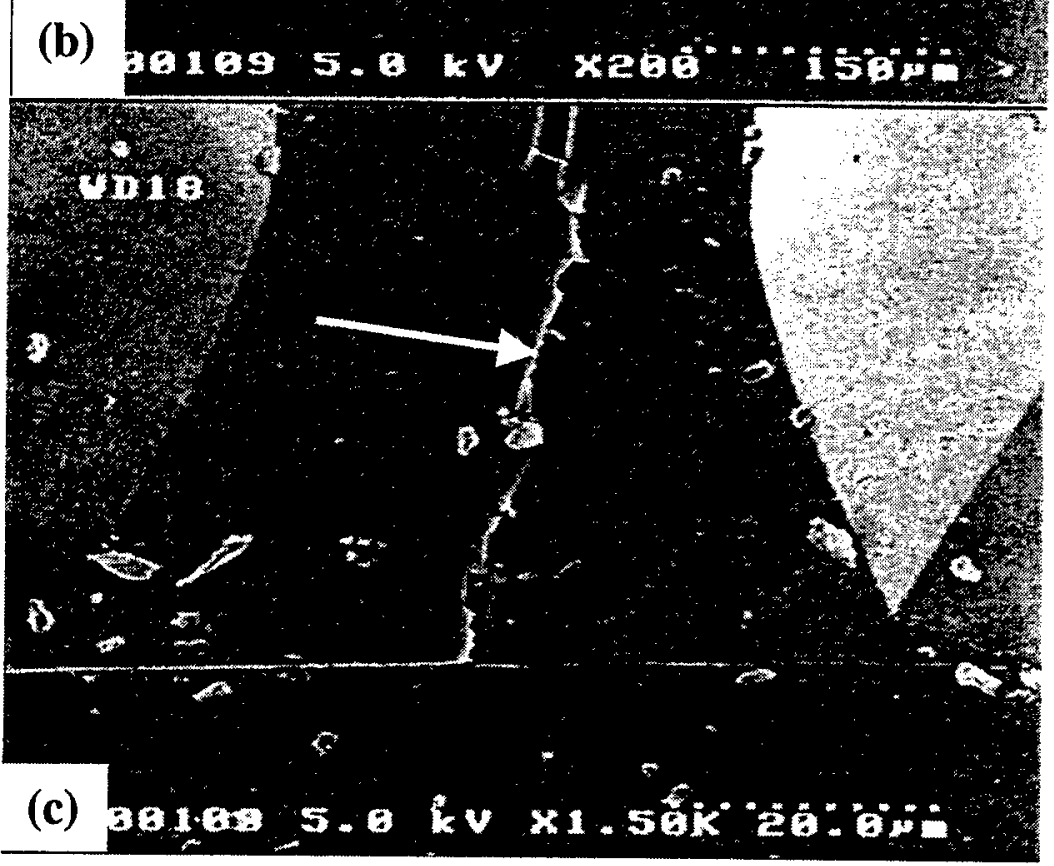
To protect the sensor from future direct impact, we added a protection shield to the top of the glass tube that surrounds the sensor. The design of this metal protection shield is shown in Figure 5.5. Sensor measurements with and without the cap showed similar dynamic behavior in the frequency range of operation $(<50 \mathrm{~Hz})$.

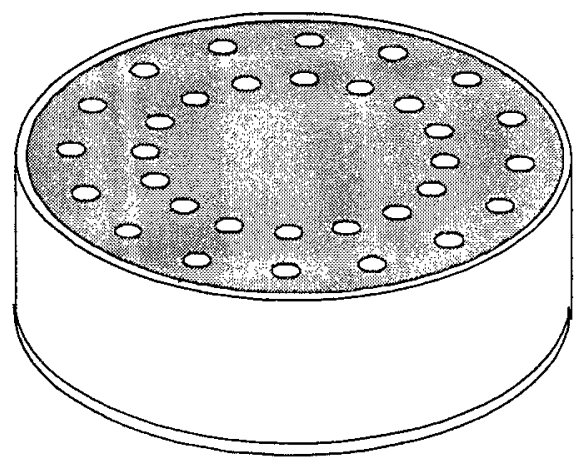

Figure 5.5. A drawing of the stainless steel cap used for protecting the MEMS device from direct impact of foreign objects.

The effectiveness of the metal protection shield was verified in the laboratory. The first test was a water jet experiment. Two prototype TBIM MCM units were prepared with only a MEMS device and the glass tube wall installed. The metal shield was applied to one unit using nonconductive epoxy while the other unit was kept as a control. Both units were exposed to a consistent water jet applied normal to the sample using identical experimental procedures. Both units were physically inspected for diaphragm integrity and their bridge resistances were measured before and after the water jet exposure. The control sample suffered complete destruction of the diaphragm, while the protected unit showed no change in performance. This is shown in Figure 5.6.

(a)

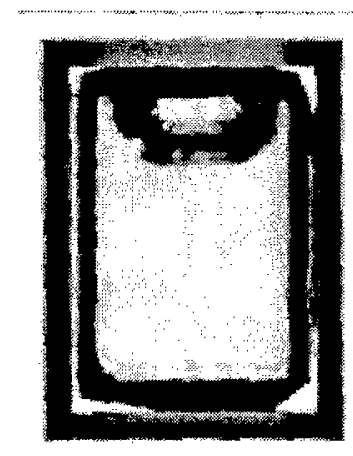

(b)

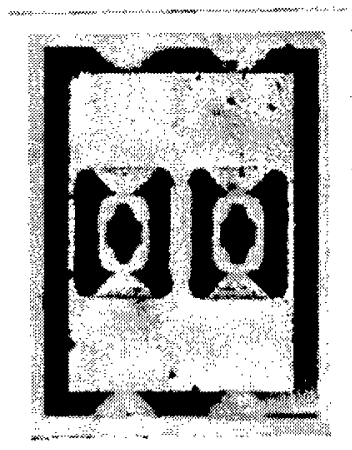

(c)

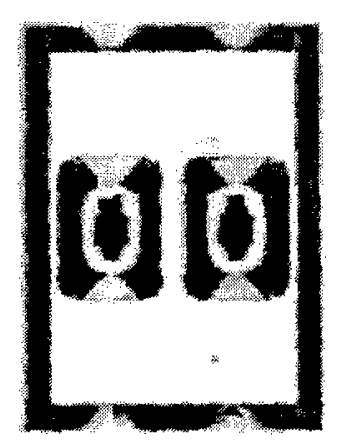

Figure 5.6. Results of laboratory experiments, testing the integrity of the MEMS protective shield: (a) Completely ruptured diaphragm due to water jet exposure, (b) Damaged BBJ test samples (hairline crack, too fine to be seen in this magnification), and (c) The protected sensor after removing the lid, showing no damage.

The protected membrane was also examined at much higher magnifications, to verify that indeed there were no hairline cracks anywhere. A second set of experiments was conducted to assess the effect of trapped, freezing moisture on this modified package configuration (with the metal shield). There was some concern that moisture may get trapped in the protected sensor area, which could give rise to deleterious effects when the aircraft was exposed to freezing temperatures. Four samples were prepared: two with the metal shield and two unshielded 
controls. The tube wall cavity of the one unshielded unit (labeled \#2) was filled with water while the other unshielded unit was kept as control (labeled \#1). Between the two shielded units, one sample (labeled \#4) was immersed in water for 10 minutes while the other (labeled \#3) was kept at ambient. This was done to simulate exposure to a rainstorm. All four units were loaded in the temperature/humidity chamber and subjected to $100 \%$ humidity, simulating continuous rain/mist environment. After a 20 minute soak in $100 \%$ relative humidity at room temperature, the chamber temperature was ramped down to $-55^{\circ} \mathrm{C}$ with a cooling rate of $3.5^{\circ} \mathrm{C} / \mathrm{min}$. This cooling rate was selected to simulate the altitude-temperature change typically encountered in a commercial aircraft during ascent. After soaking at $-55^{\circ} \mathrm{C}$ for 20 minutes, the chamber temperature was ramped up to room temperature with a heating rate of $3.5^{\circ} \mathrm{C} / \mathrm{min}$. All samples were physically inspected and their bridge resistances were measured and recorded before and after the trapped moisture freezing experiment. At the beginning, we planned to focus on the results of samples \# 1,2,3, expecting failure of the \# 4 sample. To our surprise, all four samples survived this freezing experiment. Little change in bridge resistances was observed. It appears that even though the shielded unit (\# 4) was immersed in water, it still contained a trapped air pocket since our pressure holes are small and water surface tension may not allow any water penetration into the tube. This trapped air appears to have prevented any diaphragm damage during the freezing experiment. A photograph of a unit having the metal protective shield is shown in Figure 5.7.

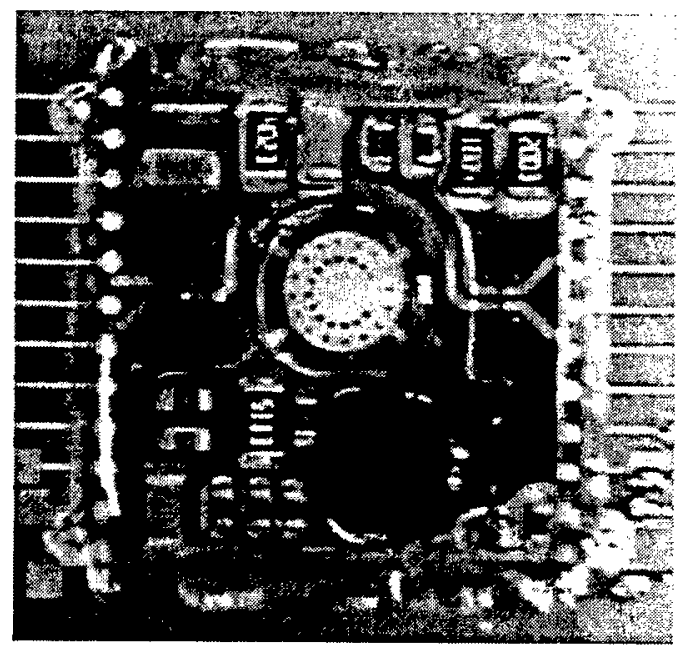

Figure 5.7. A photograph of a TBIM MCM with the metal shield installed.

\subsection{Electronic Component Packaging}

The ICs are bonded to the MCM using conductive epoxy. The electrical connections between the ICs and the MCM pads are done with gold wire-bonding. Finally, the devices are encapsulated in a glob-top epoxy, which only extends around each device. If the epoxy covered the entire MCM it would exert too much stress onto the substrate, especially at lower temperatures, which in turn could contribute to a spurious pressure reading. The passive devices, resistors and capacitors are attached to the MCM pads using conductive epoxy or solder reflow. More details on the protective coatings and epoxy development are given in Section 5.4. 


\subsection{Multichip Module Packaging}

The multichip module contains all the circuitry of the TBIM. It is made of a silicon substrate with copper traces for conductors, encapsulated in a thin titanium layer, and polyimide as the interlayer/intermetal dielectric. We use solid-filled vias between the different metal layers, which are also made of copper encapsulated in titanium. The I/O pads on the MCM are made of copper/titanium/nickel/gold and are suitable for wire-bonding, TAB or flip-chip bonding. There were two distinct MCMs made, each for a specific purpose

As discussed earlier, we followed a parallel path in the development of TBIM. One was suitable for flight testing that had the form and fit of the final hardware, which would allow us to develop and debug the packaging technology. The second one was to develop and test the functional capability of the hardware in the laboratory. We refer to these two designs as the analog and digital versions of the pressure belt. The first path led us to the development of a prototype TBIM, shown in Figure 5.8, that incorporated some of the desired functionality of the final belt but more importantly, had the same form factor, materials, and packaging as the final version. We will refer to this prototype module as the "analog" MCM module. The reason is its output is a $0-5 \mathrm{~V}$ analog signal that scales with the measured pressure in psi. This module does not have a bus interface, so each analog TBIM had to be wired separately. However, it was very suitable for flight testing the various packaging configurations, materials, and processes. As one can see in Figure 5.8, the MEMS device can be connected to three equivalent sites. It was initially deemed that perhaps the MEMS sensor reading depended on its location on the MCM (upstream or downstream) but this became a non-issue when we decided to use a fairing over the belt, since the fairing eliminated direct exposure of the belt to the air stream.

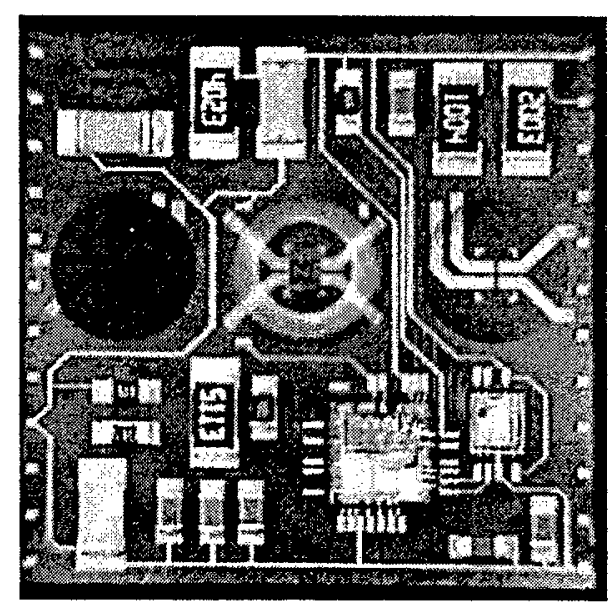

Figure 5.8. Photograph of the assembled analog MCM module used for flight testing (without the protective coatings applied).

The fairing was designed so the pressure belt did not disturb the aerodynamics at the boundary. The use of the fairing was motivated by a concern on the part of the aerodynamicists that unless the belt had a perfectly smooth aerodynamic surface, it could disturb the measurement. Early in the program, we felt this could be adequately addressed by incorporating a coating that created a smooth surface over the whole belt. However, due to a number of reasons this was not deemed very practical or desirable. On the other hand, there was enough evidence in the literature (See for instance, "The Effects Of Transducer Flushness On Fluctuating Surface Pressure 
Measurements" in AIAA $2^{\text {nd }}$ Aero-Acoustics Conference, Richard Hanley, NASA Ames, 1975) that the use of a fairing could accomplish this objective. In addition, a fairing would provide a means for handling the belt and providing extra protection for the various components. So, based on these requirements, a fairing was designed (using different materials) for this purpose. An engineering drawing of the final version of the fairing is shown in Figure 5.9.

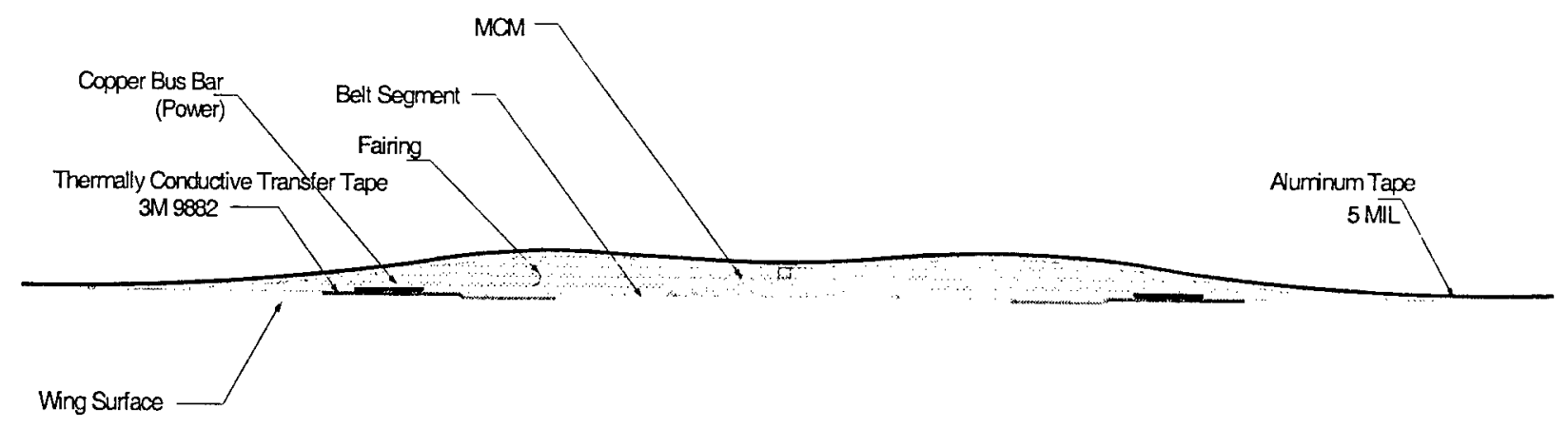

Figure 5.9. A cross-sectional drawing of the fairing design.

Each analog MCM is 0.8 by 0.8 -inch in size, roughly the same size as the final (digital) MCM. Since the location of the MEMS device on the MCM was not critical, it was decided to locate only one device in the center, connected to the electronic circuitry. The electronic circuitry uses an MCA 7707 chip. The MCA7707 (equivalent to MAX1457, available through MAXIM) is an analog signal conditioning processor optimized for piezoresistive sensors. Additional components include an EEPROM and passives, such as resistors and capacitors with various values and tolerances. A pyrex tube is used to isolate the MEMS device from the protective coating used on the rest of the MCM. These coatings protect the IC.s from environmental influences; they are discussed in detail in the next section. The pyrex tube also protects the MEMS device from any handling damage during assembly and installation. A picture of an analog pressure belt segment with six modules is shown in Figure 5.10.

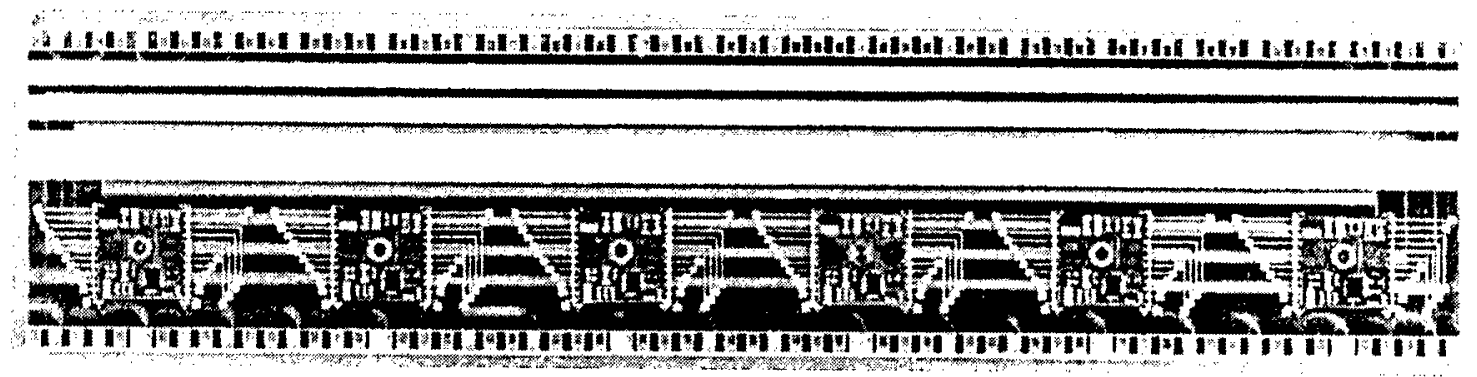

Figure 5.10. A picture of the analog pressure belt used for flight testing.

Prototype belt segments with MEMS transducer elements in die form were provided for laboratory evaluation, prior to flight testing. They were evaluated through the following tasks:

1. Characterization of the MCA7707 (MAX1457) signal processor for temperature compensation of each operable MCM onboard the prototype belt segments

2. MCM performance evaluation throughout the design operating ranges of pressure and temperature 
3. Collection of baseline MEMS performance data throughout design operating ranges of pressure and temperature

4. Calculation of surface fit coefficients and demonstration of accuracy capability

5. Repetition of testing cycles to determine stability and reliability

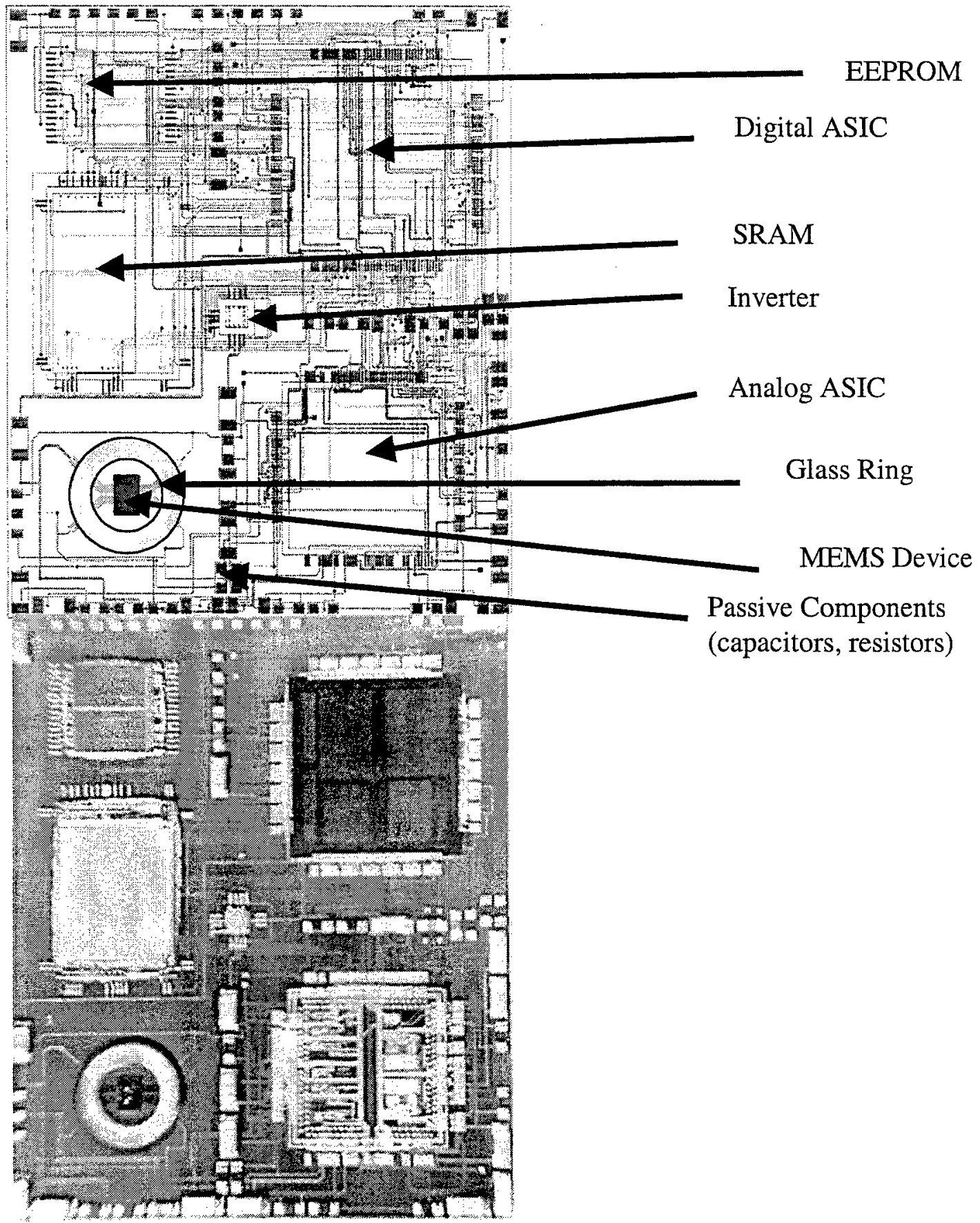

Figure 5.11. The layout of the digital TBIM MCM and a photograph of an assembled part (without the protective coatings applied). 
The size of the digital MCM is roughly 1.1 by 1.3 inches. A layout of the MCM and a picture of the finished module, which includes 39 components, are shown in Figure 5.11. This same analog module was used for all the flight tests, as it was easy to use and easier to troubleshoot if it failed. The success of these experiments validated the materials and processes used in the MCM fabrication and assembly in a relevant environment. Also, since the laboratory version of the TBIM was thoroughly characterized and debugged, it was time to migrate the digital TBIM design into an MCM version. The digital version of TBIM circuitry was designed using a planar MCM fabrication process with four dielectric layers. The number of interconnect layers on the MCM was kept to a minimum, so that their presence did not create undue strains on the silicon substrate, which could result in a false pressure measurement.

\subsection{Protective Coatings Development}

At the beginning of the program, the plan was to use thin coatings to protect the various components on the TBIM from environmental conditions. This plan was adopted because we knew we could not use conventional packages, if we were to meet the program requirements. The purpose of the protective coatings was to address the following requirements:

- Protection in $100 \%$ relative humidity

- Operational temperature -55 to $80^{\circ} \mathrm{C}$

- Protection from solvents, airplane fuel, hydraulic fluid

The complete requirements are listed in Section 2.2. After we started working on the program, however, it became apparent that even if a material(s) met these requirements, it was not sufficient. In addition, the protective coatings could not create any large degree of stress on the substrates they were applied to because those stresses could then be coupled to the MEMS sensor and result in erroneous readings. This meant that one of the attributes of the coatings was to have a low storage modulus. In addition, it was deemed desirable that the protective coatings be able to provide a certain degree of planarization and smoothing on the TBIM surface and ultimately on the belt segment for aerodynamic performance.

Several combinations of materials were evaluated for this purpose. Coatings could have single, double or triple layer configuration. A single layer is obviously the simplest configuration, from a manufacturing point of view. As a result, all of our early work focused on the development of a single layer coating that met all the requirements. After an initial evaluation, it became clear that no single layer material could meet all the requirements. Thus, we decided to break down the problem into manageable parts to meet all the desired properties. To meet the aerodynamic smoothing requirement it was decided to use a suitable fairing. This is common practice in flight test articles and the users were quite willing to adopt this solution, as discussed in Section 5.3. To address the issue of humidity and solvent protection, we settled on a combination of a globtop epoxy (Hysol Dexter 4460) for the protection of active electronic components (ICs) from mobile ion migration and solvents, followed by an overlayer of a low stress, siloxane elastomer (DC1-2577 from Dow Corning) for humidity protection. This configuration and combination of materials was used for the protection of the analog MEMS modules, which served as our flight test articles, as discussed in the previous section. Although the siloxane elastomer overcoat met most of our requirements, it has, like most siloxanes, a rather low resistance to jet fuel exposure. Thus, our goal shifted as we progressed in the program, in trying to improve the properties of a siloxane elastomer in terms of solvent resistance. The material identified as a suitable candidate 
was Dow Corning Q1-4939 because it has good mechanical properties well below $-65^{\circ} \mathrm{C}$ (low storage modulus). In the following Sections, we discuss some of the work done in the modification of this material. Additional information related to this work can be found in references 1-4 in Appendix A. The analytical techniques used in the characterization of material were an attempt to understand its polymerization mechanism and thermomechanical properties. The choice of techniques was based on years of experience in this field. Data derived from such tests have been used in the past for the interpretation of material properties and for the understanding of how a material performs under various environmentally stressing conditions. The analytical techniques used are described below as well as some of the experimental results obtained.

Measurement of the Glass Transition Temperature $\left(T_{g}\right)$ is important because the mechanical properties of a material change drastically at this temperature. Most polymer materials examined had a $\mathrm{T}_{\mathrm{g}}$ within the temperature range of this application $\left(-55\right.$ to $\left.80^{\circ} \mathrm{C}\right)$, which is undesirable. For instance, if a coating material has a $\mathrm{Tg}$ of $-45^{\circ} \mathrm{C}$, such as most polydimethyl siloxanes, then at $-55^{\circ} \mathrm{C}$, the modulus will increase as much as two to three orders of magnitude, which means the coating material will no longer have the desired low stress properties.

The Storage Modulus indicates the rigidity or stiffness of a material and the Loss Modulus represents how a material dissipates energy, or in other words how much energy a material can absorb. A material with a lower storage modulus means it has good flexibility and is less stiff, which allows twisting and bending of the pressure belt without generating excess stress on the coated module. The ratio of the loss modulus to the storage modulus is called the Loss Tangent. Surface Insulation Resistance (SIR) is the resistance to moisture and mobile ions that cause electrical circuit corrosion. Thus, low SIR leads to better electrical insulation characteristics.

The typical curing temperature for these materials is around $150^{\circ} \mathrm{C}$. This temperature is compatible with all the materials on the module build and it also takes a reasonable processing time. However, lower curing temperatures, including room temperature cure, were evaluated, since lower temperature processing widens the possible material choices that could be incorporated in the module build, even though this usually translates into longer curing times. Various platinum catalysts were evaluated to lower the curing temperature.

\subsubsection{Thermal Dynamic Analysis Of The Modified Siloxane Elastomers}

Differential Scanning Calorimetry (DSC) measures the heat exchange of a material with the surrounding medium as a function of temperature. A peak in DSC indicates an exothermic reaction. Here the exotherm is associated with the curing temperature. Figure 5.12 shows the effect of adding a catalyst in the curing of the Q1-4939 siloxane polymer.

The standard curing schedule for the Q1-4939 polymer is $150^{\circ} \mathrm{C}$ for 2 hrs. Curing in this polymer involves a two-component hydrosilylation polymerization, which generally takes place at elevated temperatures. Based on general practice, we felt that to lower the curing temperature it was necessary to use a catalyst to achieve this reaction. To that end, we studied the effect of three different coordinate-solvent-dissolved platinum catalysts. We found that indeed this combination can initiate the hydrosilylation at much lower temperatures, almost down to room temperature, with curing times comparable to the normal schedule. 
The three catalysts used were: 1) Platinum divinyltetramethyl siloxane, in a xylene solution (PC072), 2) Platinum divinyltetramethyl siloxane, in a linear vinylsilicone (PC075), and 3) Platinum divinyltetramethyl siloxane, in a vinyl cyclic carrier (PC085). The three catalysts have the following relative curing rates: $\mathrm{PC} 072$ (formulation 1) is a fast cure catalyst, $\mathrm{PC} 075$ (formulation 2) is a medium/fast cure catalyst, and PC085 (formulation 3) is a slow cure catalyst. Since the three platinum complexes work somewhat differently, their ability for acceleration varies. Theoretically, the onset and peak of the curing profile of the PC085, PC075, and PC072 doped resin should shift the curing exotherm in the graph to the left in the order listed, i.e. to lower temperatures, as compared to that of the base resin. However, the peak curing temperature of Formulation 2 (Base resin $+P C 075$ ) appears to be a bit higher than that of Formulation 3 (Base resin $+P C 085+$ silane). The reason is believed to be the addition of the silane adhesion promoter in Formulation 3, which accelerates the polymerization process. The addition of the silane adhesion promoter (in very small quantities) was necessary to get a well-adherent film, when using catalyst PC085.

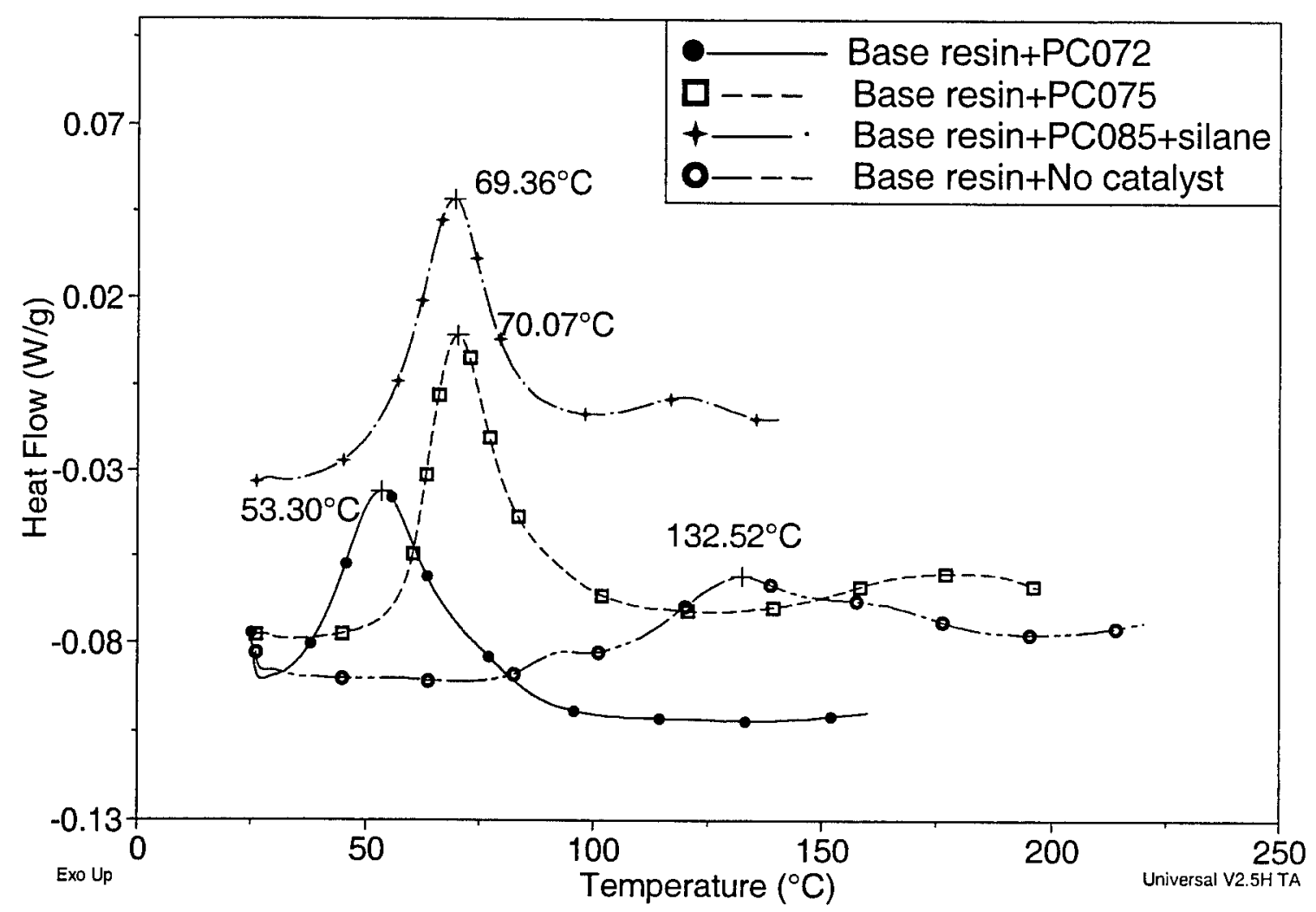

Figure 5.12. DSC comparing the curing rate of Q1-4939 using different catalysts.

Curing profile comparisons of formulations using different concentrations of the catalyst PC085 show a reduction in the peak curing temperature, but not by much. These data are shown in Figure 5.13. In addition to trying to lower the curing temperature, another objective, which was stated earlier, was to improve the chemical resistance of this polymer. An attempt to meet that objective was by loading the polymer with an inert substance. This is discussed in more detail in the next section (5.4.2). As seen in Figure 5.13, the addition of the filler tends to inhibit the polymerization and moves the peak curing temperature to somewhat higher values. 


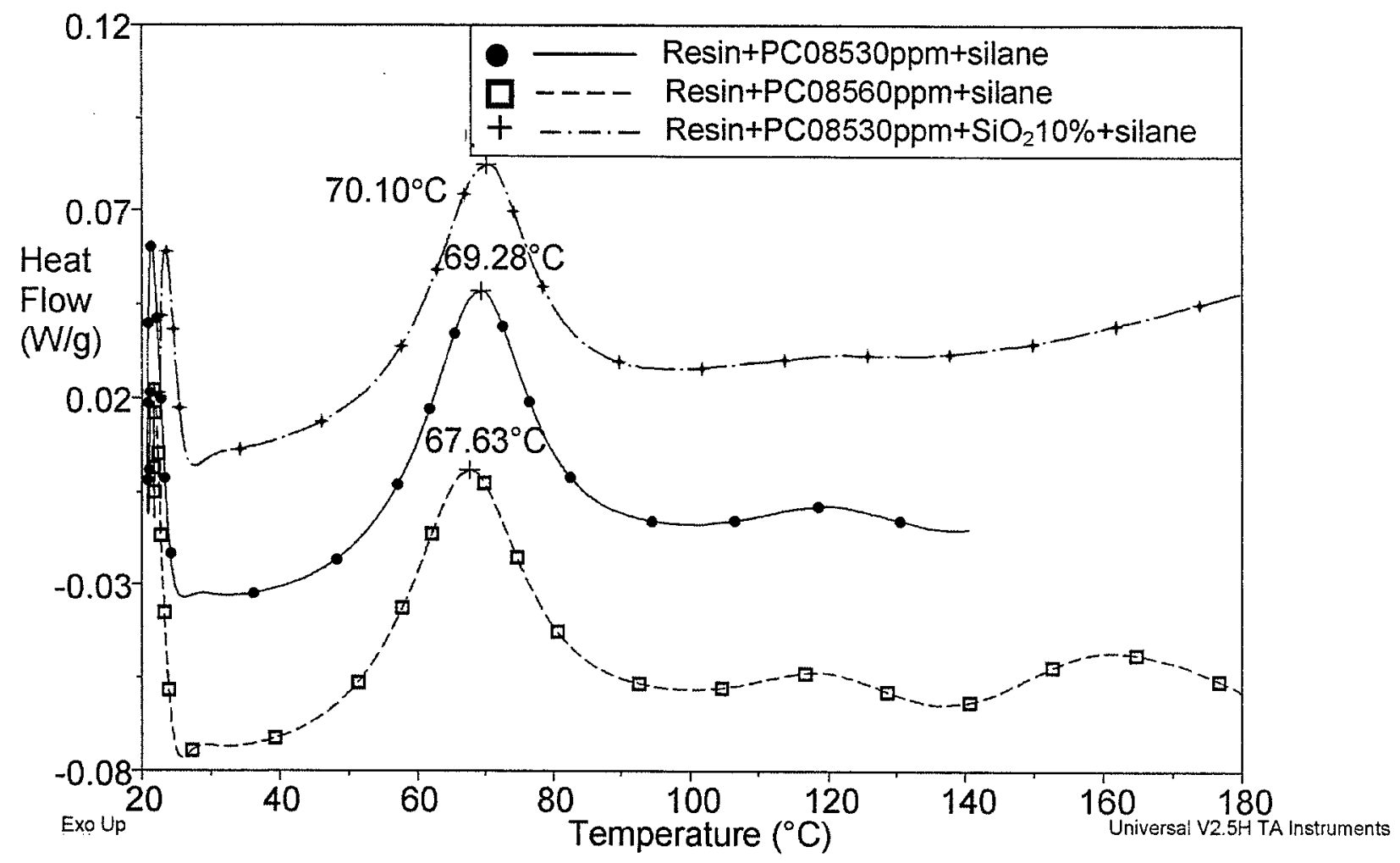

Figure 5.13. DSC curves comparing the effect on the curing temperature of Q1-4939 using different PC085 concentrations and also the effect of adding a filler.

In general, one would like to minimize the amount of catalyst used, decrease the curing time or temperature. To optimize these three parameters without any detrimental effects in the properties of the final cured siloxane conformal coating, an experiment was run using different amounts of the three different catalysts. Some of the results of these optimization experiments are shown in Table 5.1. The curing temperature for all formulations listed in Table 5.1 was room temperature, even though the base resin curing schedule is normally 2 hours at $150^{\circ} \mathrm{C}$. The results show that catalyst PC085 with a concentration of $\sim 30 \mathrm{ppm}$ and catalyst PC075 with a concentration of $20 \sim 25 \mathrm{ppm}$ appear to be the best. 
Table 5.1. Results from the optimization experiments using different catalysts

\begin{tabular}{|c|c|c|c|c|c|c|c|}
\hline \multicolumn{8}{|l|}{ Catalyst PC072 } \\
\hline & Concentration (ppm) & 45 & 31.5 & 27 & 23 & & \\
\hline & Curing time (hours) & $<0.7$ & $<1$ & $<1.8$ & $<1.5$ & & \\
\hline & Bubble formation & yes & yes & yes & yes & & \\
\hline \multicolumn{8}{|l|}{ Catalyst PC075 } \\
\hline & Concentration (ppm) & 45 & 30 & 20 & & & \\
\hline & Curing time (hours) & $<1.5$ & $<2$ & $<6$ & & & \\
\hline & Bubble formation & few & fewer & no & & & \\
\hline \multicolumn{8}{|c|}{ Catalyst PC085 (formulations include 2 wt $\%$ of silane) } \\
\hline & Concentration (ppm) & 150 & 96 & 66 & 44 & 22 & 13 \\
\hline & Curing time (hours) & $<2$ & $<2$ & $<2.5$ & $<2.8$ & $<6$ & $<8$ \\
\hline & Bubble formation & yes & yes & yes & few & no & no \\
\hline
\end{tabular}

\subsubsection{Chemical Resistance Evaluation}

As stated earlier, another objective of this activity was to improve the chemical resistance of this Q1-4939 siloxane polymer. To achieve this goal, the base resin was loaded with $\mathrm{SiO}_{2}$, an inert substance. The chemical resistance tests were done using the base resin as a control, cured at $150^{\circ} \mathrm{C}$ for 2 hours, both with or without filler loading and with or without the silane adhesion promoter. This is shown in Figure 5.14.

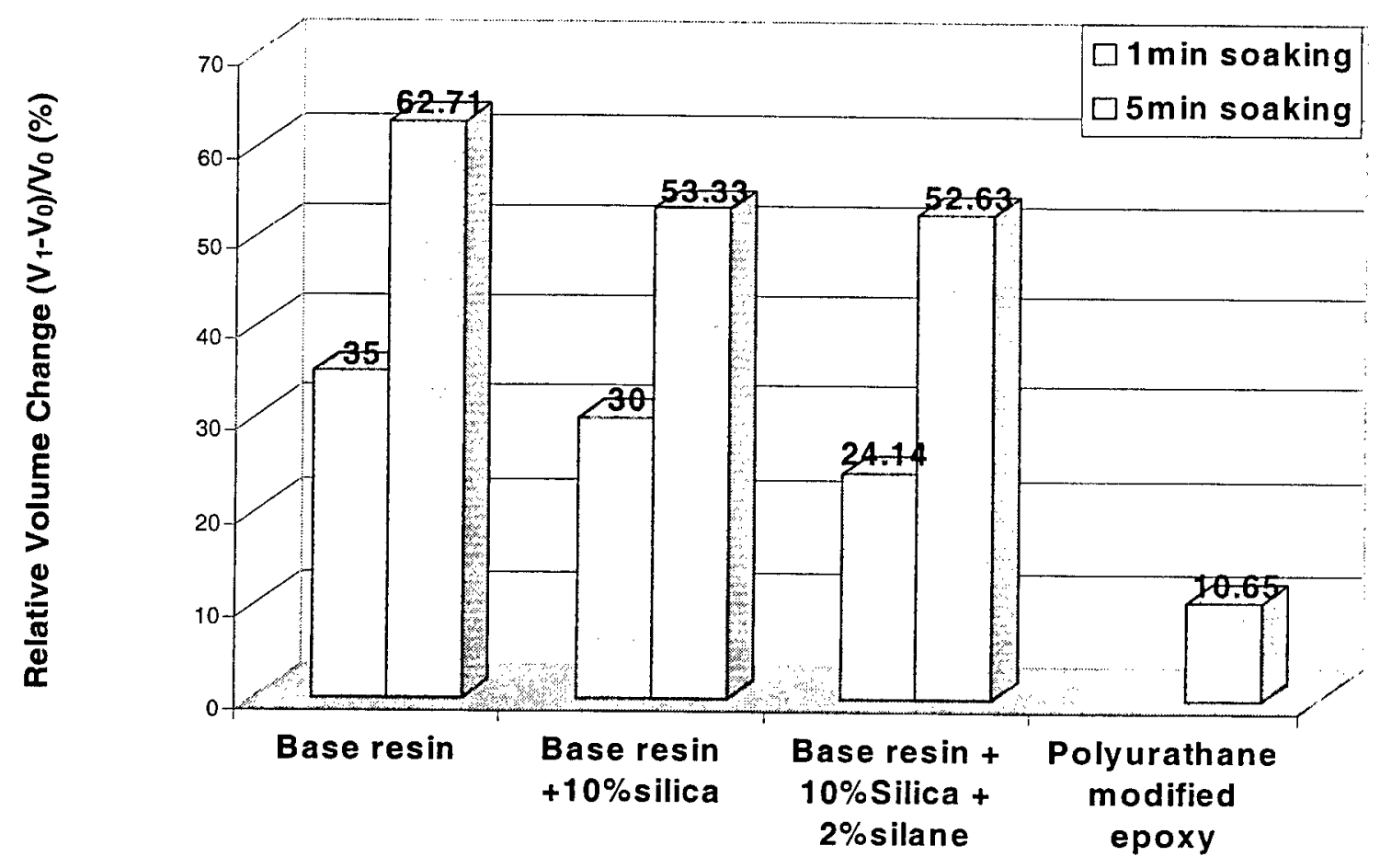

Figure 5.14. Q1-4939 swelling vs. filler loading percentage. 
The results of 1 minute immersion in gasoline indicate that $10 \%$ filler loading can reduce the swelling from $35 \%$ to $30 \%$ of the original volume. This is almost $14.29 \%$ reduction in the amount of swelling. The addition of silane further enhances the chemical resistance because of some increase in the degree of cross-linking in the bulk siloxane. This further improves the volume swelling reduction to $24.14 \%$. In Figure 5.14 the swelling property of a polyurethane modified epoxy is also included in the chart as reference because it was expected to have good chemical resistance. In that material, the swelling after a 5 minute immersion in gasoline is only $10.65 \%$ of the original volume. This is the material that we initially worked on and evaluated as a candidate for the single layer protective coating. Unfortunately, it was unsuitable for our needs because its $\mathrm{T}_{\mathrm{g}}$ is higher than $-65^{\circ} \mathrm{C}$, as explained in the beginning of this section.

We also wanted to evaluate the solvent resistance of room temperature-cured resins, with and without the $\mathrm{SiO}_{2}$ filler. The resins were cured at room temperature for 6 hours. There is a significant difference shown in Figure 5.15 for the samples with and without the filler. The catalyst used for the room temperature curing for all three samples was PC085 in $30 \mathrm{ppm}$ concentration.

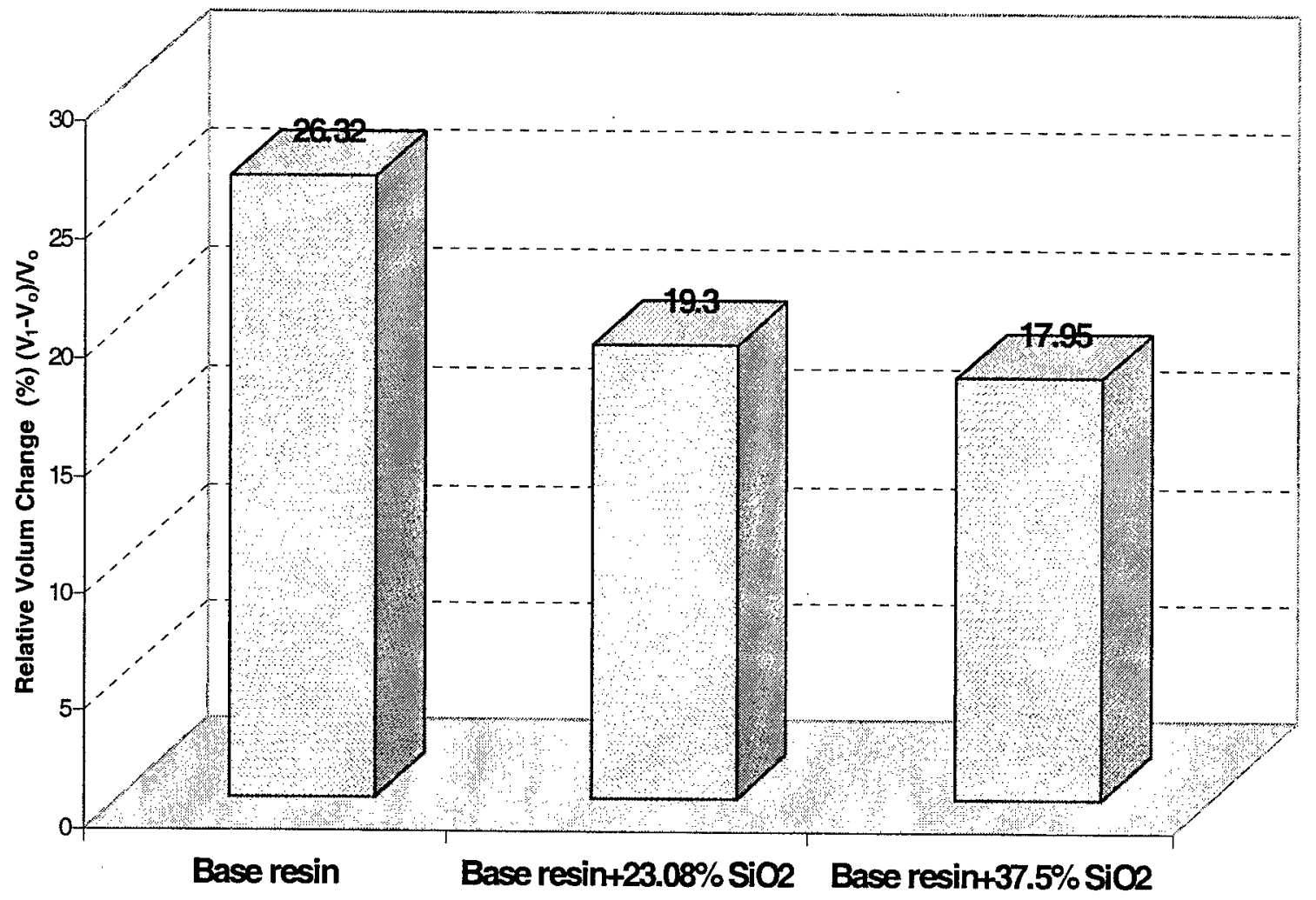

Figure 5.15. Material swelling vs. filler loading for a room temperature cured Q1-4939 resin (after immersion in jet fumes for 5 minutes) with and without a filler.

The results indicate the use of the catalyst alone can reduce the volume swelling from $62.7 \%$ to $26.32 \%$. When the filler loading is increased from $0 \%$ to $23.0 \%$ and $37.5 \%$, the volume swelling is further reduced from $26.32 \%$ to $19.3 \%$ to $17.95 \%$, respectively. Consistent with what is shown in Figure 5.14, the addition of the filler reduces the swelling of the material system because of an improvement in the oil-philic property of the polymer and the cross-linking 
density. In general, the trend of chemical resistance improvement vs. filler loading shows an exponential (bowl shape) decrease. Actually, there is a tradeoff between chemical resistance and surface insulation resistance (SIR), which relates to moisture uptake. There is only a small degradation in SIR for the formulations with improved chemical resistance and it is within acceptable limits.

\subsubsection{Mechanical Properties Characterization}

To increase the resistance of the coating to jet fuel, an inorganic filler $\left(\mathrm{SiO}_{2}\right)$ was incorporated into the base resin system. Dynamical Mechanical Analysis (DMA) tests were performed to verify that the filler loading was not changing the storage modulus of the base materials too much. In DMA, instruments can either apply a sinusoidally varying load and measure the (sinusoidally varying) displacement, or vice versa. From the data, one can then determine the complex moduli (storage modulus and loss tangent) of the specimen. This is one of the most useful thermal analysis techniques because it can clearly identify the Glass Transition temperature and other transitions in polymer and polymer-matrix composite specimens. Using this analytical method we can determine the stiffness and damping properties of a material. Figure 5.16 shows that the $\mathrm{T}_{\mathrm{g}}$ of the series remains lower than $-120^{\circ} \mathrm{C}$, even for significant additions of the filler. Although the storage moduli of the series is higher than that of the control

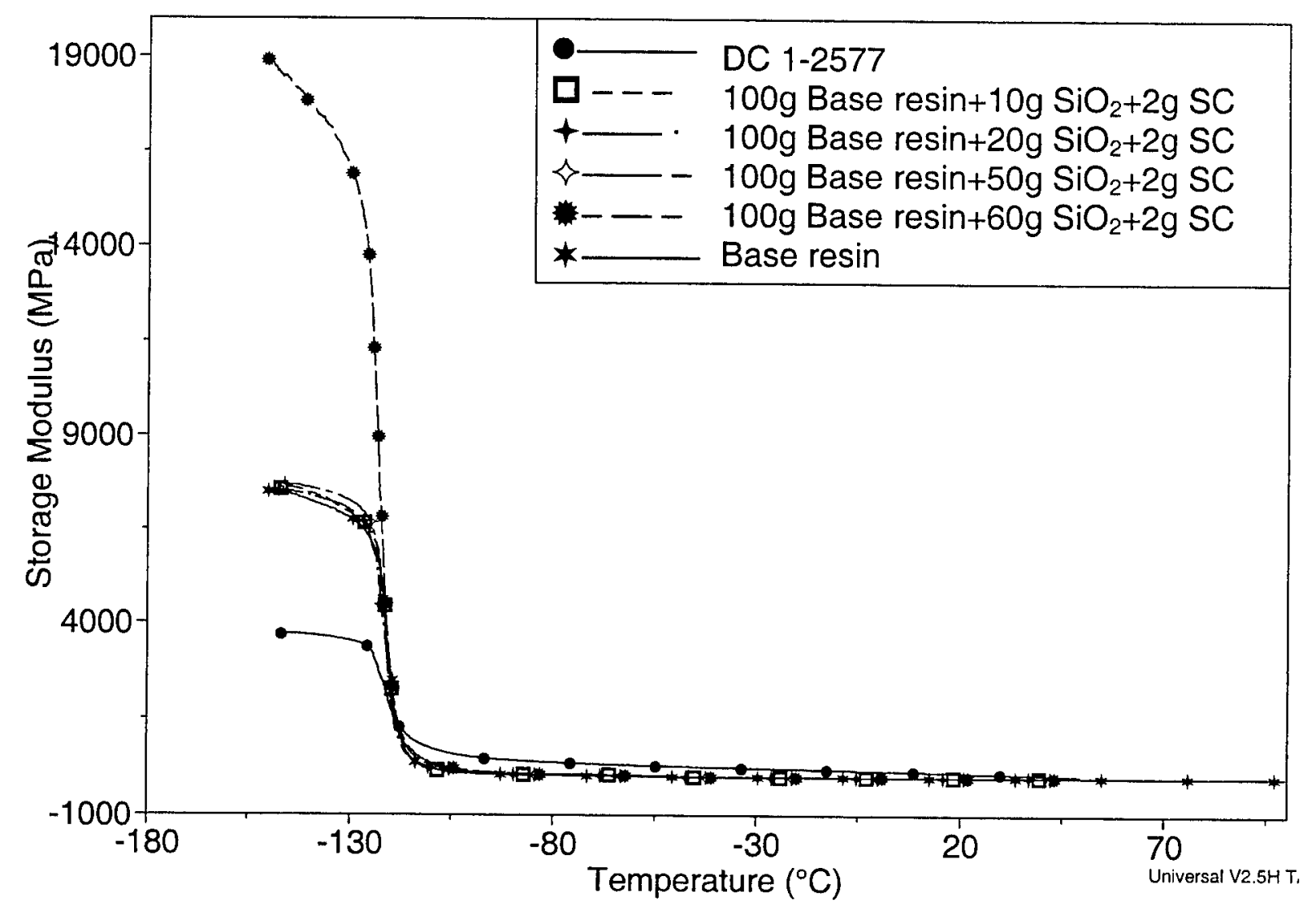

Figure 5.16. Storage modulus change of Q1-4939 as a function of the percentage of filler loaded into the resin system. SC stands for the silane coupling agent used to improve adhesion. 
sample (DC 1-2577) for temperatures below $T_{g}$, the storage moduli of the series is actually lower than that of control samples once the temperature gets higher than their $\mathrm{T}_{\mathrm{g}}$ 's. Overall, the moduli of the series meets the low internal stress requirements needed by this project.

Additionally, some room temperature-cured samples, with various combinations of filler, were tested for elongation, maximum force sustainable and toughness (work required for fracture). The results presented in Table 5.2 show that, in general, the maximum force and the toughness increase with filler loading.

Table 5.2. Room temperature cured sample properties for various filler combinations

\begin{tabular}{|l|c|c|c|}
\hline Specimen & $\begin{array}{c}\text { Elongation } \\
\left(\mathrm{L}-\mathrm{L}_{0}\right) / \mathrm{L}_{0}(\%)\end{array}$ & $\begin{array}{c}\text { Max. Force } \\
(\mathrm{N})\end{array}$ & $\begin{array}{c}\text { Work } \\
(\text { Joules })\end{array}$ \\
\hline Base resin+60wt\% $\mathrm{SiO}_{2}+30 \mathrm{ppm} \mathrm{SC}$ & 229.7486 & 12.7 & 231.74 \\
\hline Base resin+50wt\% $\mathrm{SiO}_{2}+30 \mathrm{ppm} \mathrm{SC}$ & 245.7142 & 13.5 & 235.89 \\
\hline Base resin+40wt\% $\mathrm{SiO}_{2}+30 \mathrm{ppm} \mathrm{SC}$ & 292.9451 & 9.9 & 178.94 \\
\hline Base resin+30wt\% $\mathrm{SiO}_{2}+30 \mathrm{ppm} \mathrm{SC}$ & 297.9736 & 12.6 & 233.26 \\
\hline Base resin+20wt\% SiO2+30ppm SC & 285.595 & 11.5 & 186.54 \\
\hline Base resin+10wt\% SiO2+30ppm SC & 299.3923 & 8.4 & 151.71 \\
\hline Base resin+30ppm SC & 334.9625 & 7.1 & 134.95 \\
\hline Base resin+60ppm SC & 336.3694 & 15.6 & 228.2 \\
\hline
\end{tabular}

The moduli of the siloxanes before and after immersion in a solvent were also investigated. In general, solvent exposure does not appear to affect the chemical structure of the siloxane. The $\mathrm{T}_{\mathrm{g}}$ 's of the materials before and after immersion are almost identical, whether it is the non-fillerloaded high temperature cured resin base shown in Figure 5.17 or the filler-loaded room temperature-cured resin base like the one shown in Figure 5.18.

Based on these data it appears that the low-stress, modified-siloxane-based conformal coating will have good resistance to jet fuel compared to the DC1-2577, which is currently being used as the baseline material for the top coating. The material has good rheology, it can be cured at room temperature and become tack-free within 5 hours. The modulus of the developed conformal coating (Q1-4939 based) is lower than the DC1-2577 within the operational temperature $\left(-55^{\circ}\right.$ to $\left.80^{\circ} \mathrm{C}\right)$. It also has good flexibility and durability as well as extremely low $\mathrm{T}_{\mathrm{g}}$. 


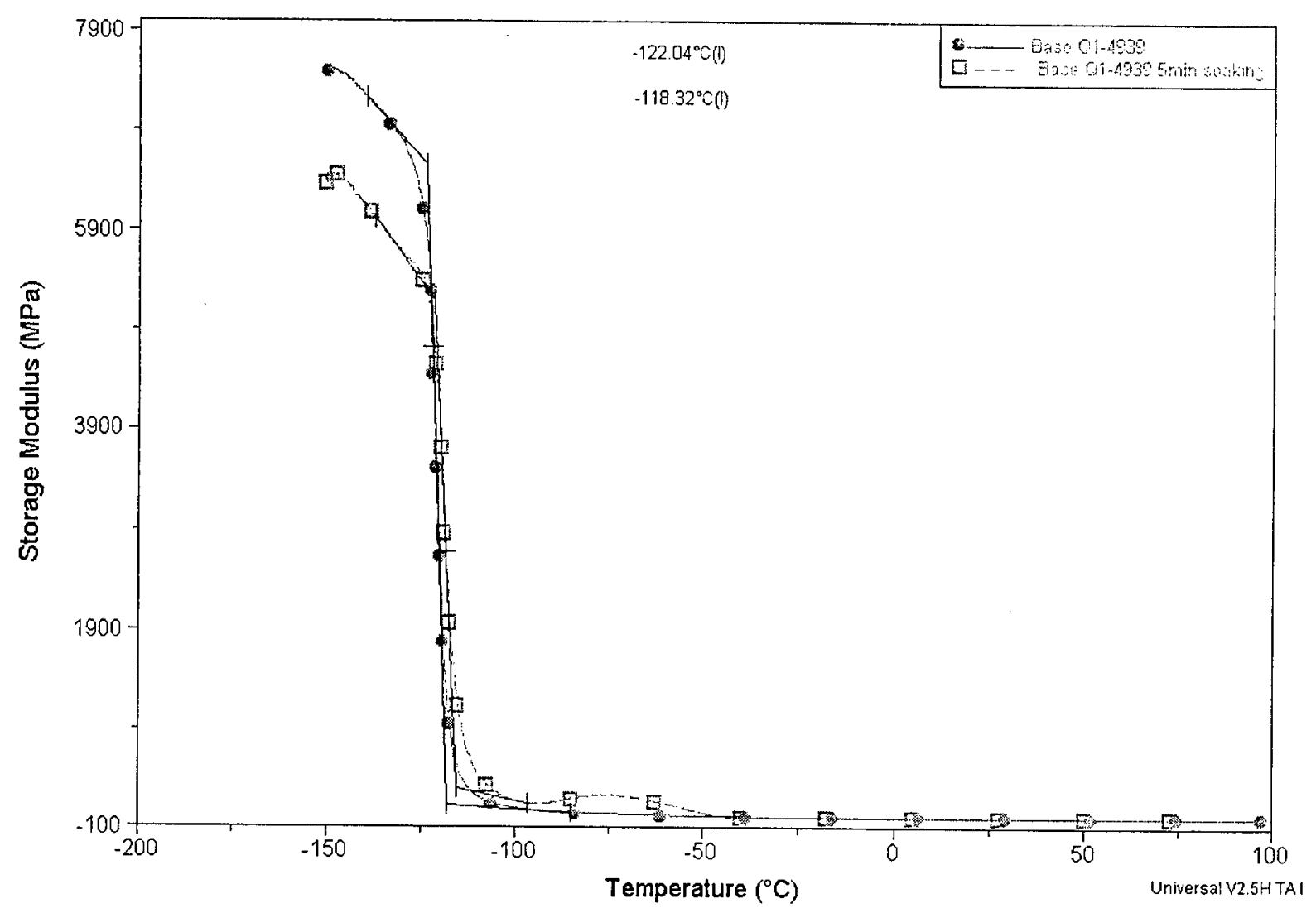

Figure 5.17. Moduli comparison on room temperature-cured Q1-4939 resin before and after soaking in gasoline for 5 minutes.

One of the reasons for choosing a siloxane polymer was its inherent resistance to humidity. So it was important that in the course of trying to improve the material properties we do not change this desirable attribute. The most common way one measures the resistance to humidity and mobile ions in a polymer material is by measuring Surface Insulation Resistance (SIR). Figure 5.19 shows the SIRs of some of the modified siloxane-based conformal coatings in $85 \%$ Relative Humidity $@ 85^{\circ} \mathrm{C}$ and $160 \mathrm{~V}$ bias as a function of time. All of the samples maintain SIRs above or close to $1 \times 10^{8} \mathrm{Ohms}$ except for one sample of Q1-4939+additive+additional cure. The choice of $R=1 \times 10^{8} \mathrm{Ohms}$ as a benchmark value for the goodness of a conformal coating is somewhat arbitrary. It was used extensively at the AT\&T Bell Labs for internal processes. It is a rather conservative value. Thus, small deviations from this value are not expected to have any impact. This is certainly true for the data shown in Figure 5.19.

In general, there does not appear to be any significant difference in the SIR of samples cured at room temperature after an additional $80^{\circ} \mathrm{C} / 15$ minute thermal curing. This implies that the samples are most likely fully cured at room temperature. This is further validated by the DSC data shown in Figure 5.20 for samples with and without a $80^{\circ} \mathrm{C} / 15$ minute post cure (sample was first cured at room temperature for 10 hours). The absence of a clear endotherm or exotherm implies that these modified siloxane coatings are already cured at room temperature, consistent with the SIR results, which show there is no significant change in the moisture uptake of the room-cured coatings vs those with a post-bake. 


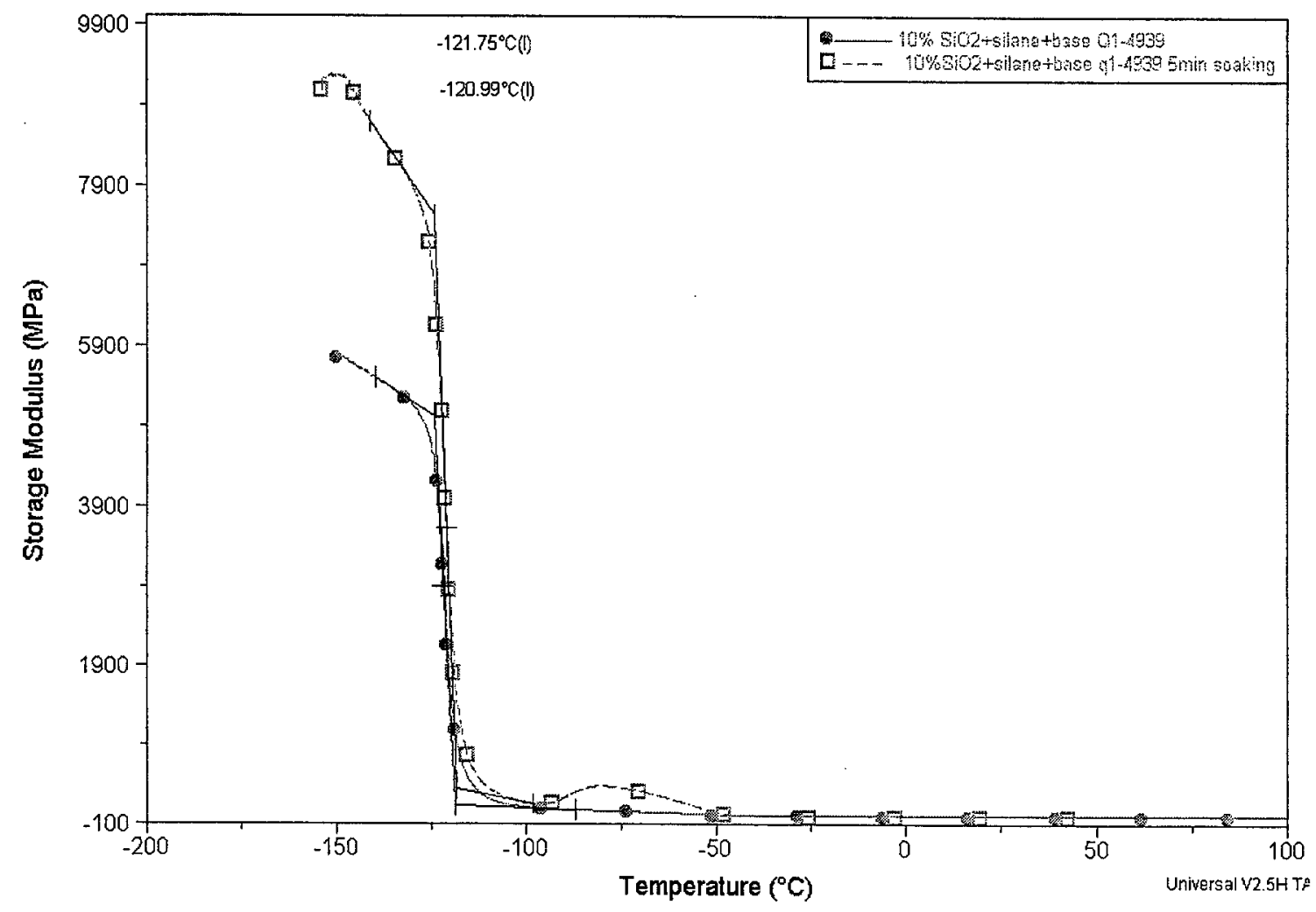

Figure 5.18. Moduli comparison on high temperature curable resin with filler loading before and after soaking in gasoline for 5 minutes.

The materials chosen for the protective coatings on the final belt are: (a) DC Q1-4939 as the low stress silicon gel at the base of the MEMS device, (b) Dexter Hysol FP4460 for glob-top on the ICs, and (c) DC1-2577 silicone coating for protecting the MCM.

Georgia Tech's research on protective coating materials resulted in a new material that can be implemented into our system as a replacement for the DC1-2577. It offers an improvement in jet fuel resistance over the DC1-2577, as discussed in the report.

However, despite its promise, this material was only demonstrated in laboratory experiments and its compatibility has not yet been verified in Boeing's manufacturing process flow. Furthermore, most of the new results were obtained towards the end of the program, so it was too late to insert it into our current pressure belt manufacturing activities. Actual implementation of this material will require additional engineering work to qualify it for manufacturing, including some associated tool and fixture design. For now, we intend to use the DC1-2577 material as the overall material coating system, even though it does not have the superior properties that the Georgia Tech material has, because it is compatible with our current manufacturing processes and has adequate jet fuel resistance for the current round of flight testing. Furthermore, it has proven its reliability through multiple flight tests on commercial and military jets. We expect that the next pass of the pressure belt to incorporate this new material system as a protective layer. 


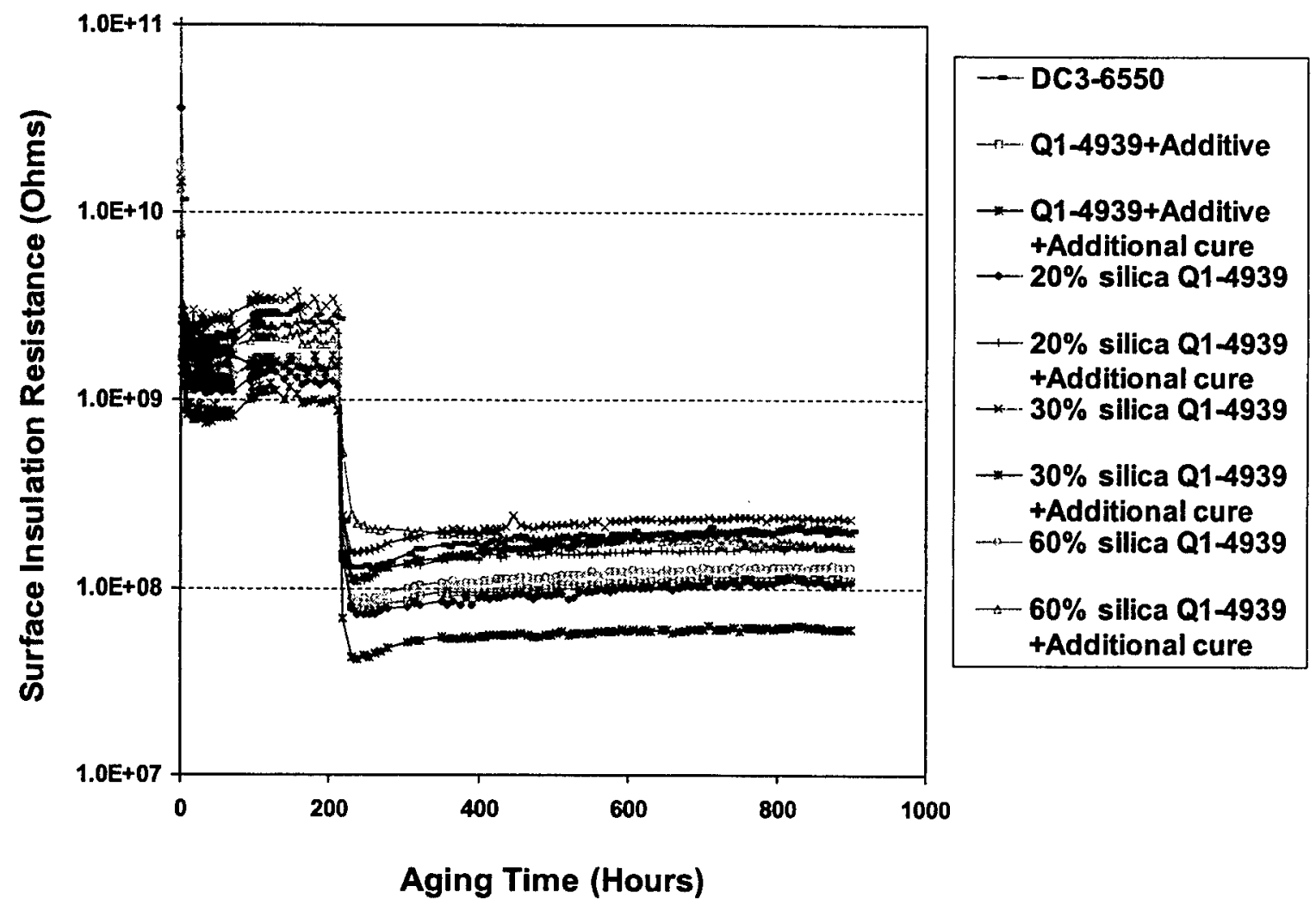

Figure 5.19. Surface insulation resistance of candidate coatings vs. aging time under $85 \%$ relative humidity $@ 85^{\circ} \mathrm{C}$ under $160 \mathrm{~V}$ bias (in a Triple Track Test configuration).

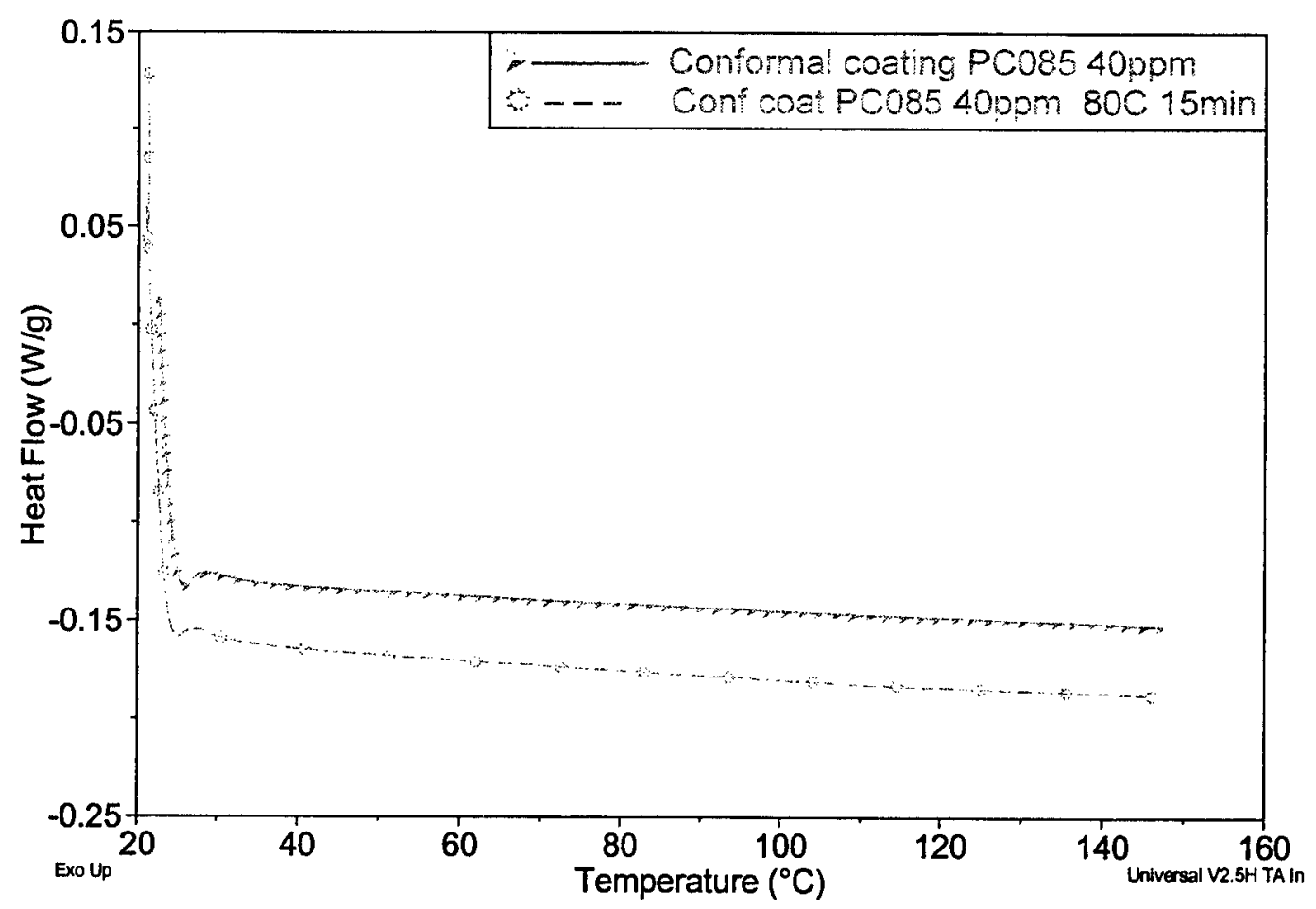

Figure 5.20. DSC results on samples with and without $80^{\circ} \mathrm{C} / 15$ minute post cure. 


\subsection{Pressure Belt Segment Packaging}

Early in the program, the tape material for the pressure belt segments was based on TAB (Tape Automated Bonding) technology. However, as the complexity of the circuitry on the segment grew, it became apparent that a TAB-based tape would be extremely expensive and would only be available from a limited number of suppliers. For this reason we decided to migrate our design to a flex circuit tape. Flex circuits with two layers are widely available at reasonable cost from many suppliers. The down side of this choice was looser manufacturing tolerance. This was deemed acceptable, even though it made segment assembly a bit more complicated.

The flex circuit-based segment has a length of 12.46 inches and contains $6 \mathrm{MCM}$ locations. As explained earlier, all locations do not have to be populated at all times. This fact also provides fault-tolerance to the pressure belt design. A schematic of the segment design is shown in Figure 5.21. The flex circuit tape consists of two 0.0014-in thick ( $1 \mathrm{oz}, 35$ micron) copper layers on both sides of a 0.002 -in thick polyimide support with dielectric overlays ( 0.002 in thickness including adhesive, if any) on both sides.

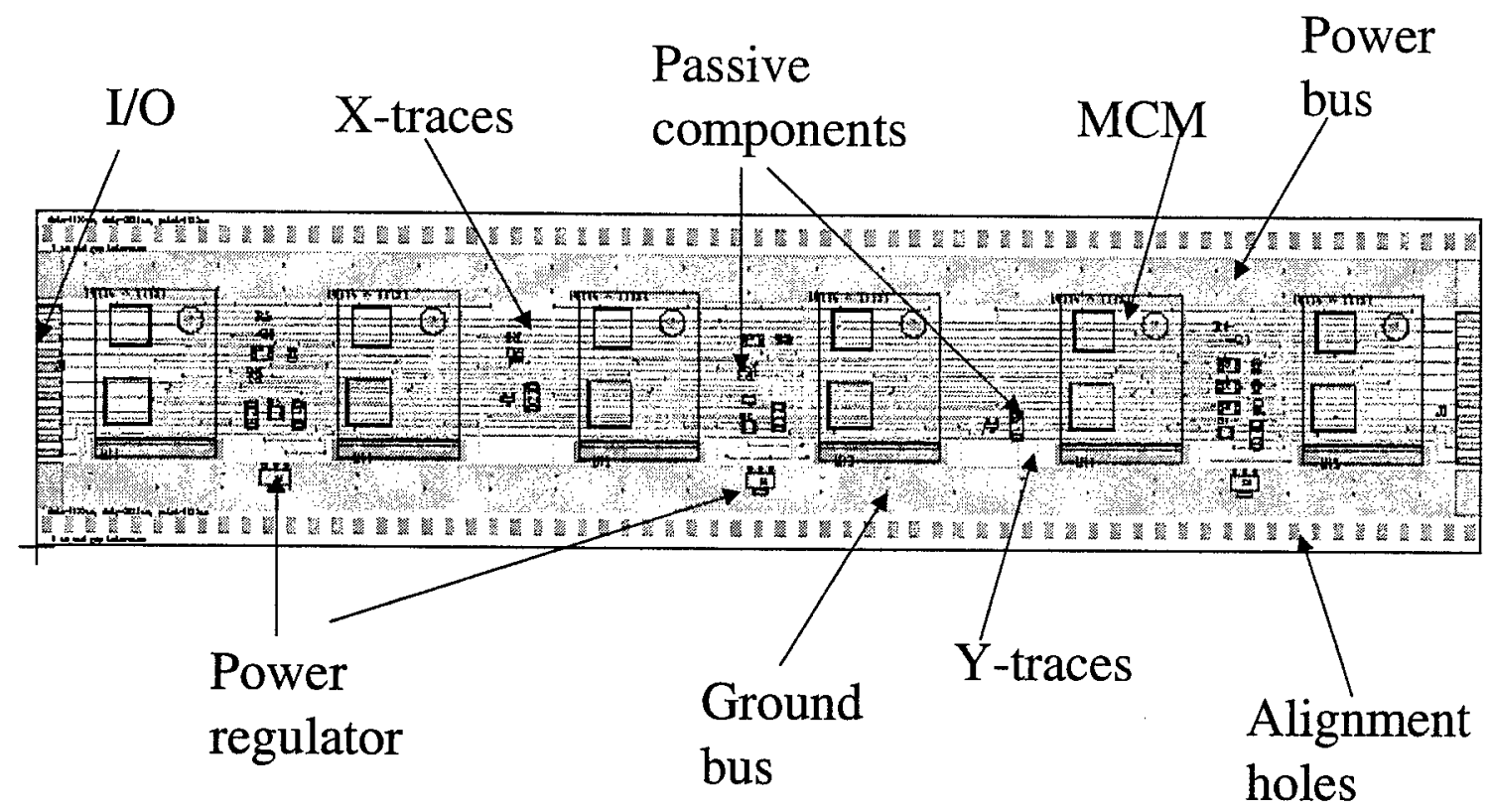

Figure 5.21. Schematic of segment design showing the various features of the pressure belt.

The top layer contains landing sites for all the components, including the power regulators, bus circuit elements, and discretes. It also contains interconnection tab features at both ends of the segment unit. The bottom conductor was used for routing. Vias were formed to connect from the top to the bottom using conventional flex circuit manufacturing processes, such as drilling and plating. Additional redundant vias were incorporated for power and ground. The overall stack was laser cut to create openings in the flex circuit with a tolerance of less than 2 mil in reference to its corresponding MCM unit center. The triple " $u$ " shaped tongues are used for the next level assembly as wrap-around interconnections. The sprocket holes located at the edges are used for mechanical alignment during the assembly process. They are also used as a reference mark for locating the MEMS sensors in regards to the airplane wing. All copper surfaces are protected with either $\mathrm{Sn}$ or a Ni/Au layer, which also makes the surface solderable. 
The flex circuitry supplier was Cirexx. A photograph of the assembled segment is shown in Figure 5.22.

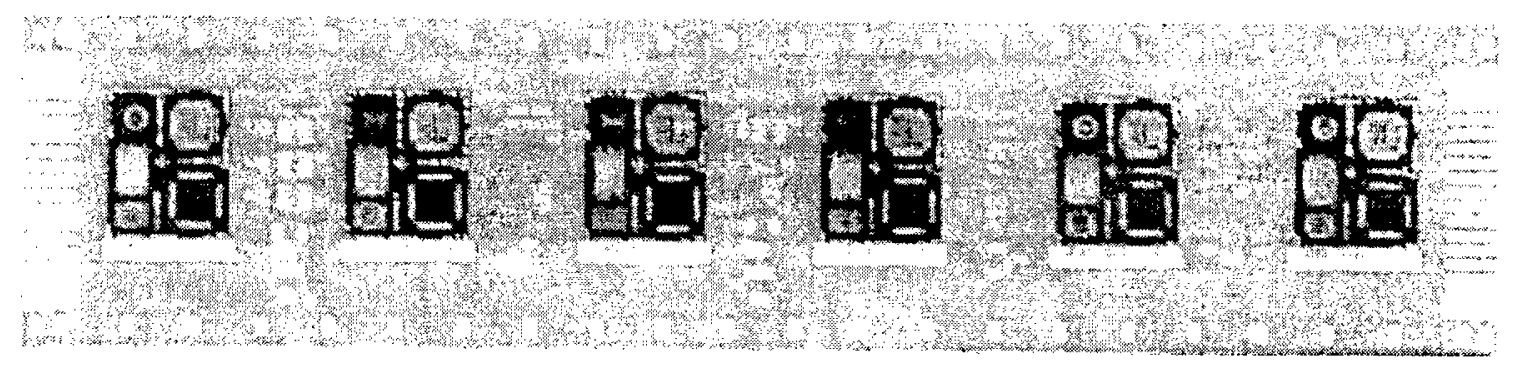

Figure 5.22. Photograph of an assembled pressure belt segment. 


\subsection{FLIGHT TESTING}

To validate the technology developments generated by this program, many tests were performed. Most of the testing took place in the laboratory. However, since one of the objectives of the technology development was to radically improve state-of-the-art flight testing instrumentation, it was important to demonstrate various prototype versions of this technology in a relevant environment before declaring success. In general, flight testing is a very expensive test and it is usually done for such purposes as certification or aerodynamic validation of a major design change. They are typically scheduled a long time in advance. Thus, to validate the prototype instrumentation, we had to "piggyback" on existing flight testing activities.

The first flight test that used MEMS on the wing of an airplane was performed at the beginning of the program. Endevco designed a simple circuit card-based multisensor system, which used the Endevco 8515 sensor in its conventional packaged form. The boards were interconnected to each other using twisted pair wiring. This system was in some ways similar to the analog version of the pressure belt that was used for the rest of the flight tests. The objective here was to evaluate how the sensor faired under flight test conditions. In these tests, while the sensor survived, there was a significant failure mode associated with the circuit boards. This was related to corrosion effects, due to the exposure of the whole system to rain showers for over six hours. In Figure 6.1, a picture of the early test vehicle mounted on the wing of a 737-700 is shown. Figure 6.1(a) shows the conventional tube-based pressure belts and 6.1(b) shows a closeup view. The early version of the analog pressure belt is mounted next to the conventional tubes and can be seen in Figure 6.1(b). The green rectangles are the circuit boards and the little white dots are the packaged MEMS sensors.

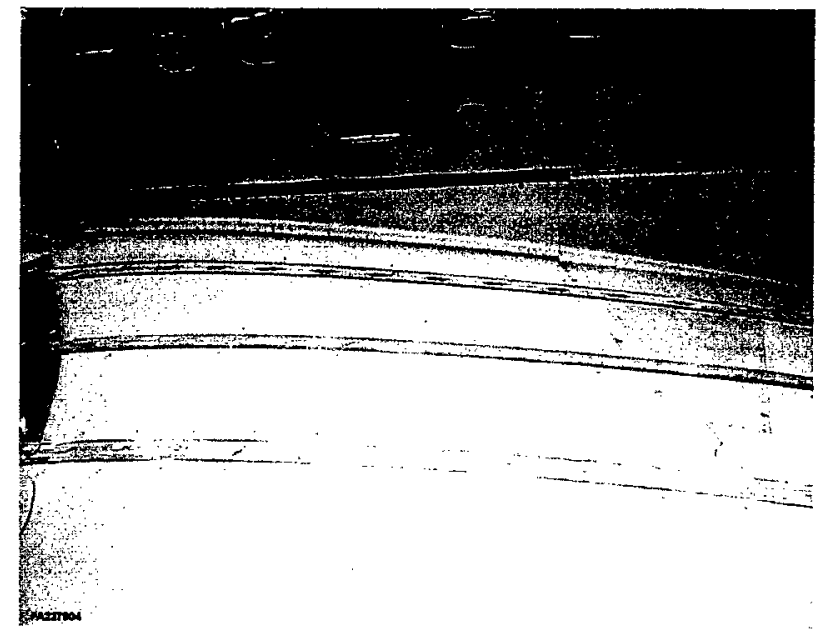

(a)

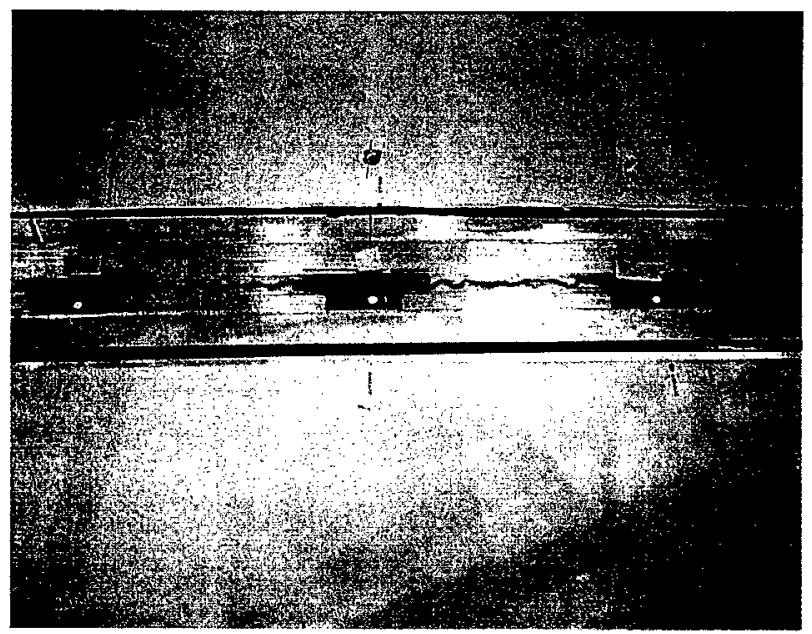

(b)

Figure 6.1. Photographs of the 737-700 wing, which was undergoing flight loads testing.

The first flight test of the analog pressure belt, which was discussed in section 5.3, using the silicon multichip modules attached to a Kapton segment, was conducted towards the end of 1998. The vehicle was a 757-300 airplane undergoing flight tests prior to certification. Our pressure belt was attached on the belly section of the aircraft, close to the landing gear doors, and 
is shown in Figure 6.2. The white section is the fairing and the surrounding gray area is the "speed" tape used for attachment.

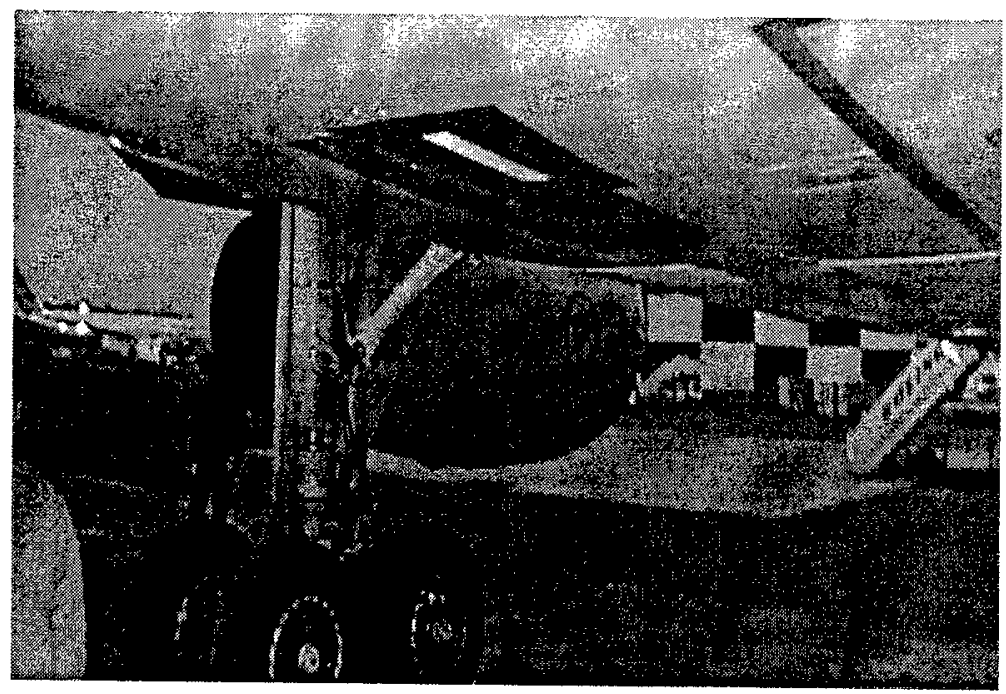

Figure 6.2. Photograph of the pressure belt attached to the belly of the 757-300.

A Honeywell PPT transducer ( 0 to 20 psia range) was installed in an instrumentation rack forward of the main landing gear. A 30 foot plastic tube extended from this pressure transducer's port and terminated at a location adjacent to the analog pressure belt as seen in Figure 6.3. As one can see, both the plastic tube and our pressure belt have aerodynamic fairings. The routing of the plastic tube (conventional pneumatic system) and the wiring (our analog pressure belt) are shown in Figure 6.4. The plastic tube snakes around the orange (test) wire and exits the picture at top left, continuing deeper into the airplane. The tube was sealed at one end and a hole was drilled in the tube at a position close to the center of the prototype pressure belt. This arrangement was intended to provide a pressure reference for comparison with the data collected from the MCM devices of the analog pressure belt.

The total volume of the PPT pressure port includes both the transducer internal volume and the volume of the plastic tube. This volume acts as a filter of the dynamic pressures occurring at the source approximately 30 feet away. In contrast, the MEMS sensors are directly exposed to the pressure source and are thus capable of responding to dynamic pressure changes in real time (resonant frequency of the MEMS devices is $\sim 180 \mathrm{KHz}$ ). Some data smoothing is appropriate in order to properly interpret performance of the MCM devices when comparing to the Honeywell PPT reference transducer. For example, we averaged 30 data points collected from the PPT and the 5 operating MCM's while the 757-300 was cruising at 15,000 feet. We tried to pick a condition in which the airplane parameters were relatively constant. The time slot where the two measurements were compared is marked with a circle in the flight test data presented in Figure 6.5. The implementation of the digital filter design in the digital version of the pressure belt addresses this data smoothing requirement. 


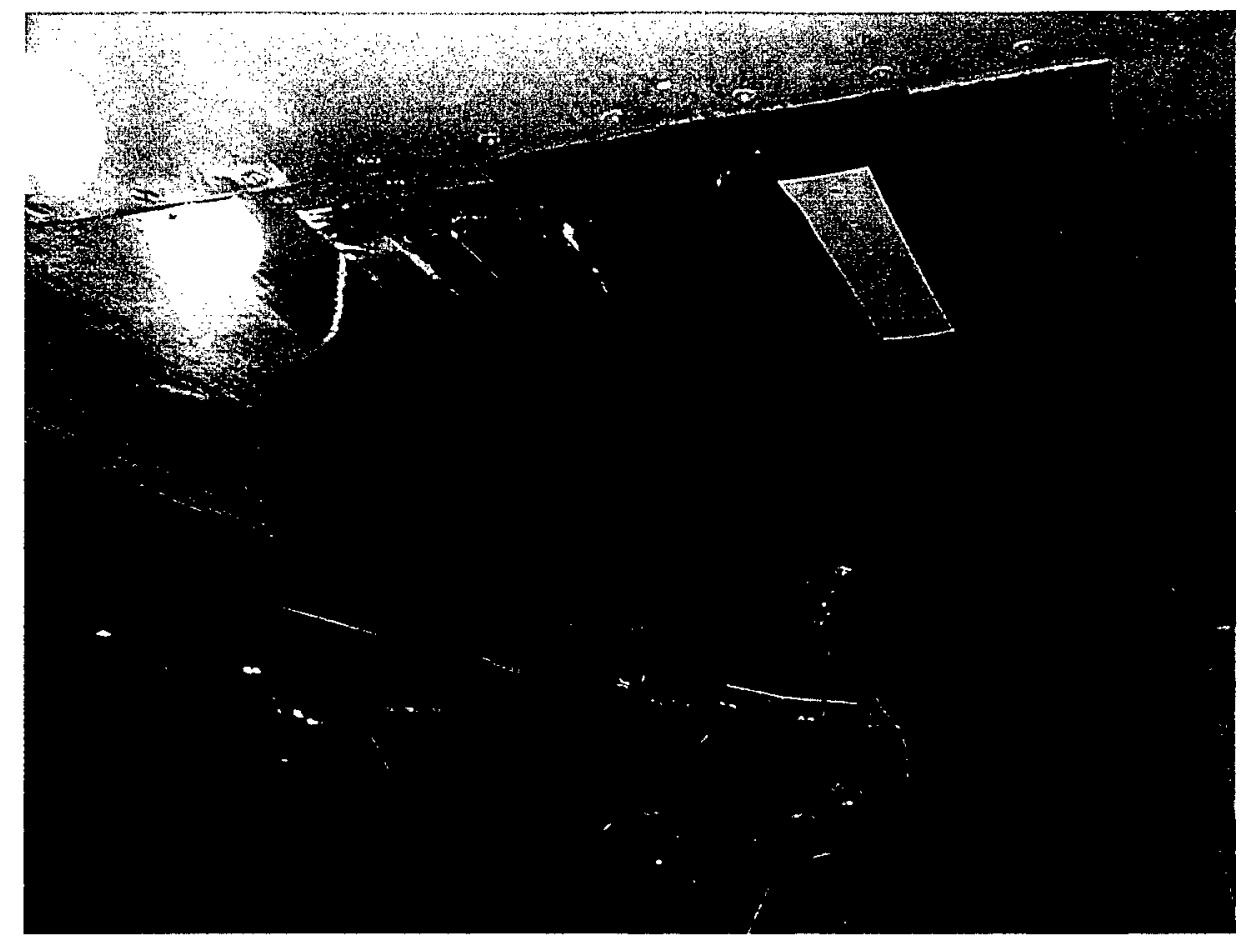

Figure 6.3. The side-by-side installation of the pressure tube and the pressure belt.

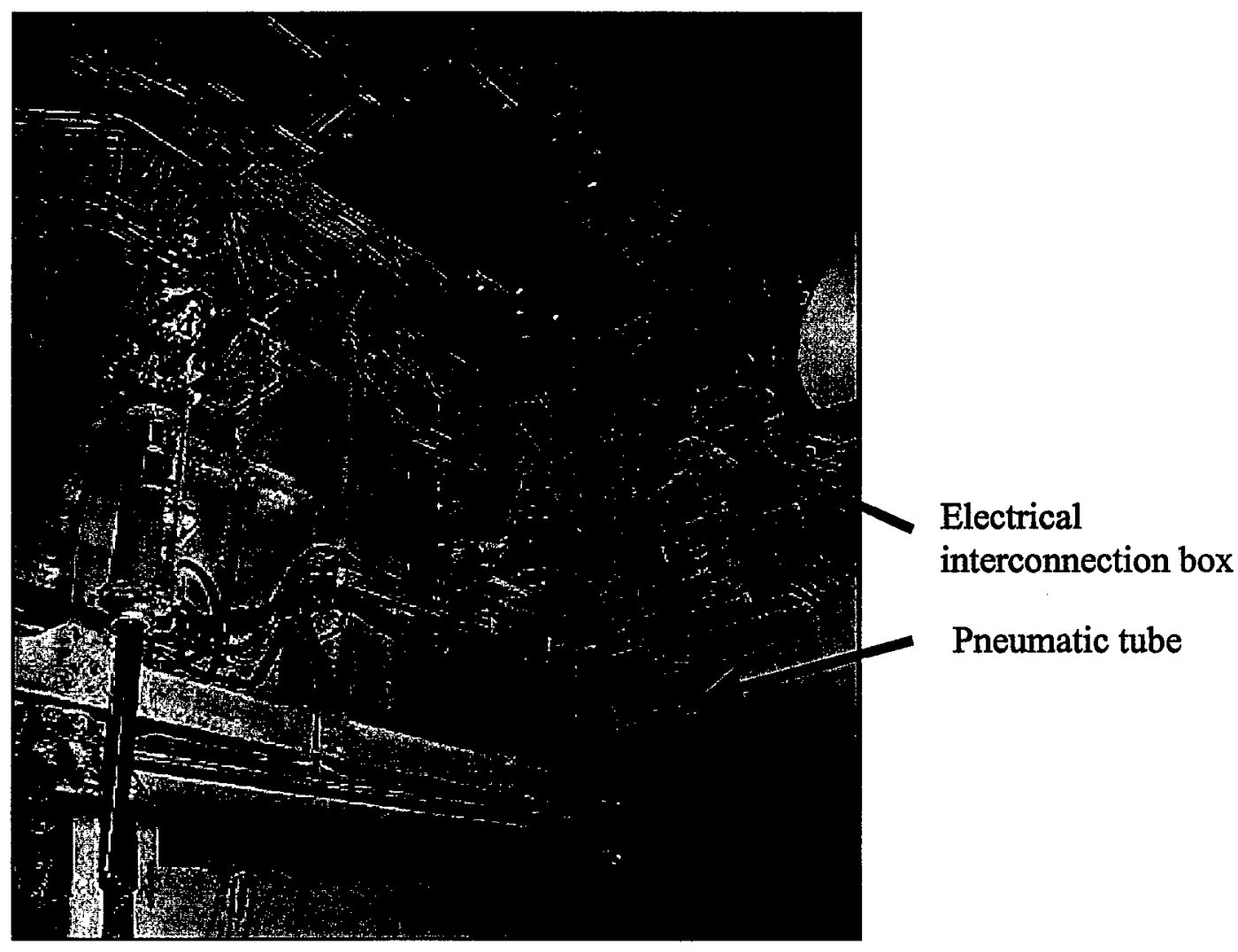

Figure 6.4. Routing of the electrical and pneumatic connections inside the landing gear bay of the 757 . 


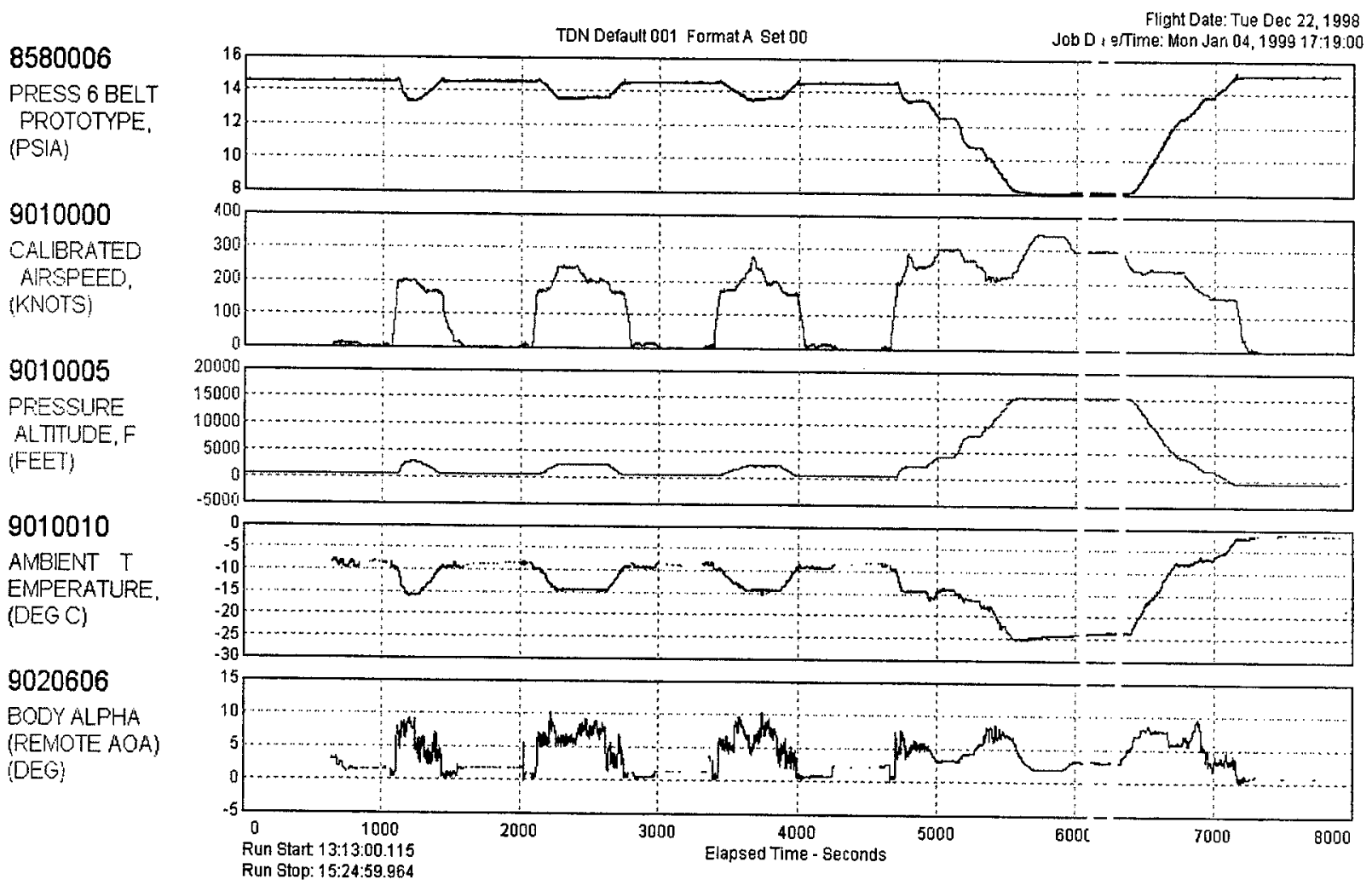

Figure 6.5. Flight test data taken on the 757-300 airplane.

The MEMS sensor data was taken along with a variety of other flight-significant parameters, such as airspeed, altitude, ambient temperature, and body alpha (angle between the airplane fuselage axis and the horizontal direction). The circled time interval was used to compare the pressure measurement between the reference sensor and the five MEMS pressure measurement channels (each analog MEMS module has one pressure measurement channel).

The percentage of full scale deviations from the averages between the PPT pressure readings (reference) and the MCMs are shown in Figure 6.6. The time elapsed for each measurement was 6 seconds ( 5 samples/second, data acquisition for a total of 30 data points) while the altitude was maintained within a couple of feet. In all, we are only plotting 19 such measurements, although many more were taken. Except for MCM02 the averaged data samples fall well within the target of $0.1 \%$ of the full scale absolute pressure. The deviation in the MCM02 may be due to a drift in calibration either in the MCM02 or in the reference (PPT). 


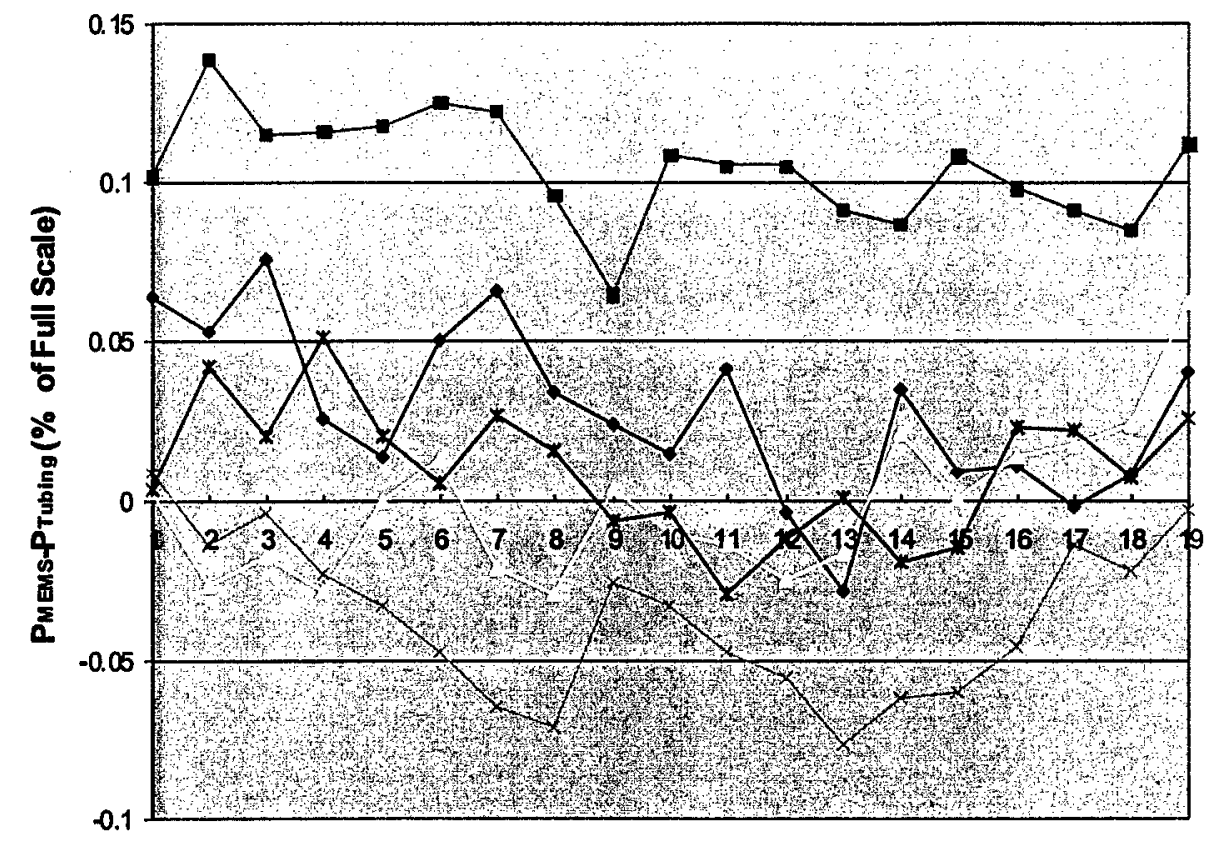

Pressure Readings of Various Sensors (Each point is an average of 30 samples)

\%F.S. Dev. MCM 01 - \%F.S. Dev. MCM $02 \quad$ \%F.S. Dev. MCM $03-\star$ \%F.S. Dev. MCM 04 - * \%F.S. Dev. MOM 05

Figure 6.6. The percentage difference between the MEMS sensor readings and the reference.

A second opportunity for a flight test of the prototype pressure belt presented itself during February 1999. This involved a 737 Boeing Business Jet (BBJ) conducting a mini-pressure survey to evaluate wing structural loads during flight. Aviation Partners Inc. installed winglets and instrumented portions of the left wing and left winglet with conventional pressure tube belts. A picture of the Boeing Business Jet is shown in Figure 6.7.

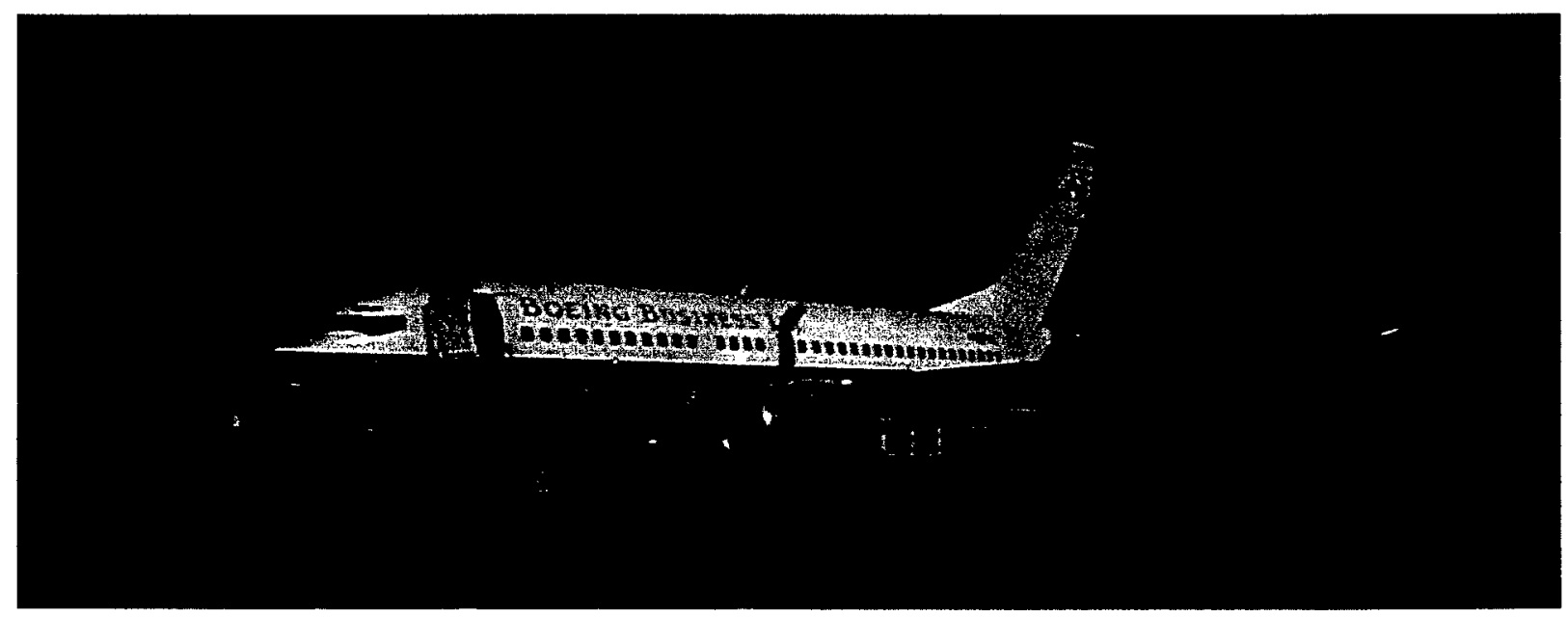

Figure 6.7. Photograph of the Boeing Business Jet ready for flight testing. 
In this airplane, the number one leading edge slat was instrumented with flush pressure ports located chordwise about 52 inches from the outboard edge of the slat. Engineering staff wanted to measure additional pressures outboard of the flush pressure ports at $35 \%$ of the slat chord. To accomplish this we installed three of the MCMs from the analog pressure belt segment that had previously flown on the belly of the 757-300.

The three MCMs were cut from the same prototype segment. Using a rapid prototyping technique also implemented for the 757-300 test, a fairing was fabricated into a 6-inch diameter disc and placed over the MCM. This was shown in Figure 5.2. Metallic tape held the fairing down onto the slat surface. Power and signal wires were routed behind and then outboard of the MCM installations, transitioning to instrumentation wiring inside the number one slat. This wiring was terminated at an instrumentation rack, located within the aircraft cabin, to provide power and signal processing for the MCMs. A picture of this installation is shown in Figure 6.8.

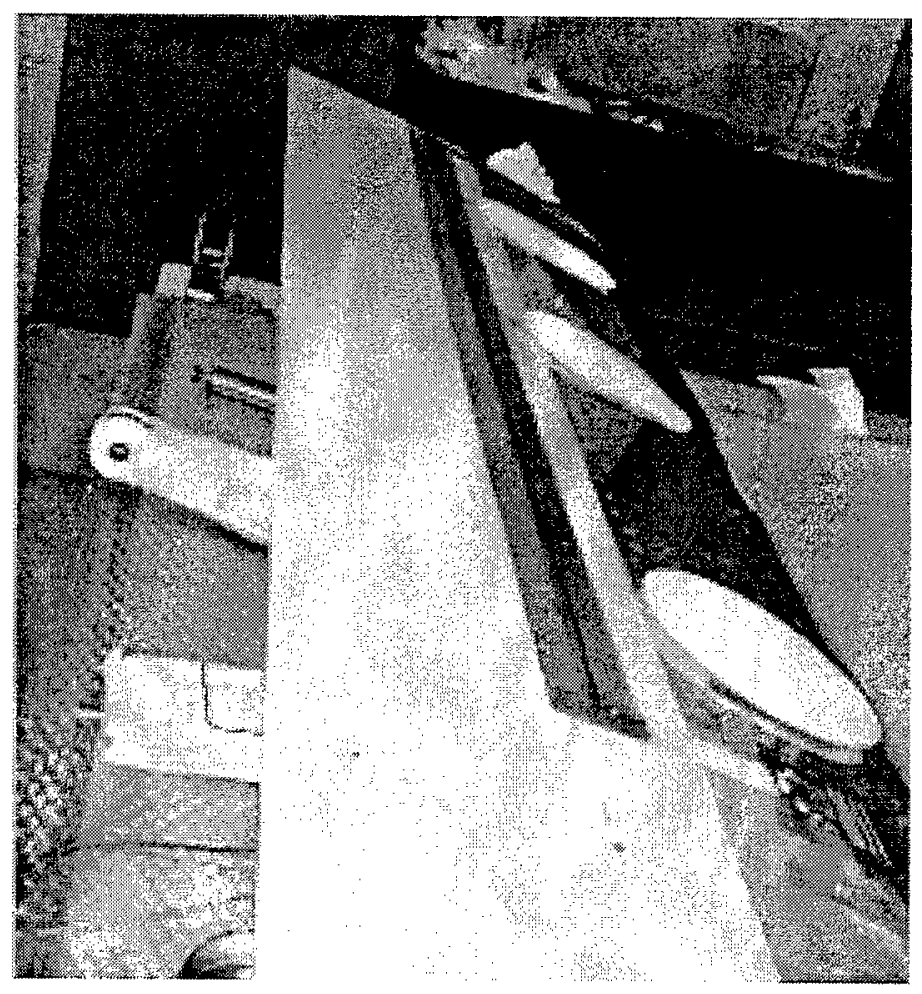

Figure 6.8. Photograph of the BBJ slat and the MEMS MCM installation.

The flight loads survey was conducted in Mesa, Arizona. Just prior to the ferry flight to Mesa, the sensor located nearest to the flush pressure ports (reference pressure) failed during a preflight test. An inquiry was made to understand the cause of the failure. Apparently, a technician had shattered the MEMS pressure sensor's diaphragm by applying a tube directly onto the sensor. The remaining two MEMS devices continued to operate throughout the flight conditions conducted during the loads survey. This unfortunate accident prevented us from comparing the flight test data gathered from the MEMS device, which was directly adjacent to one of the reference ports. Therefore, the comparison of pressure vs. time data in this test is a bit more qualitative compared with the data gathered from the 757-300 flight. The final installation on the $\mathrm{BBJ}$, prior to departure is shown in Figure 6.9. The light blue colored tape protects the reference 
ports and the MEMS access holes on the fairings. The conventional tubing belts can be seen at midspan and beyond.

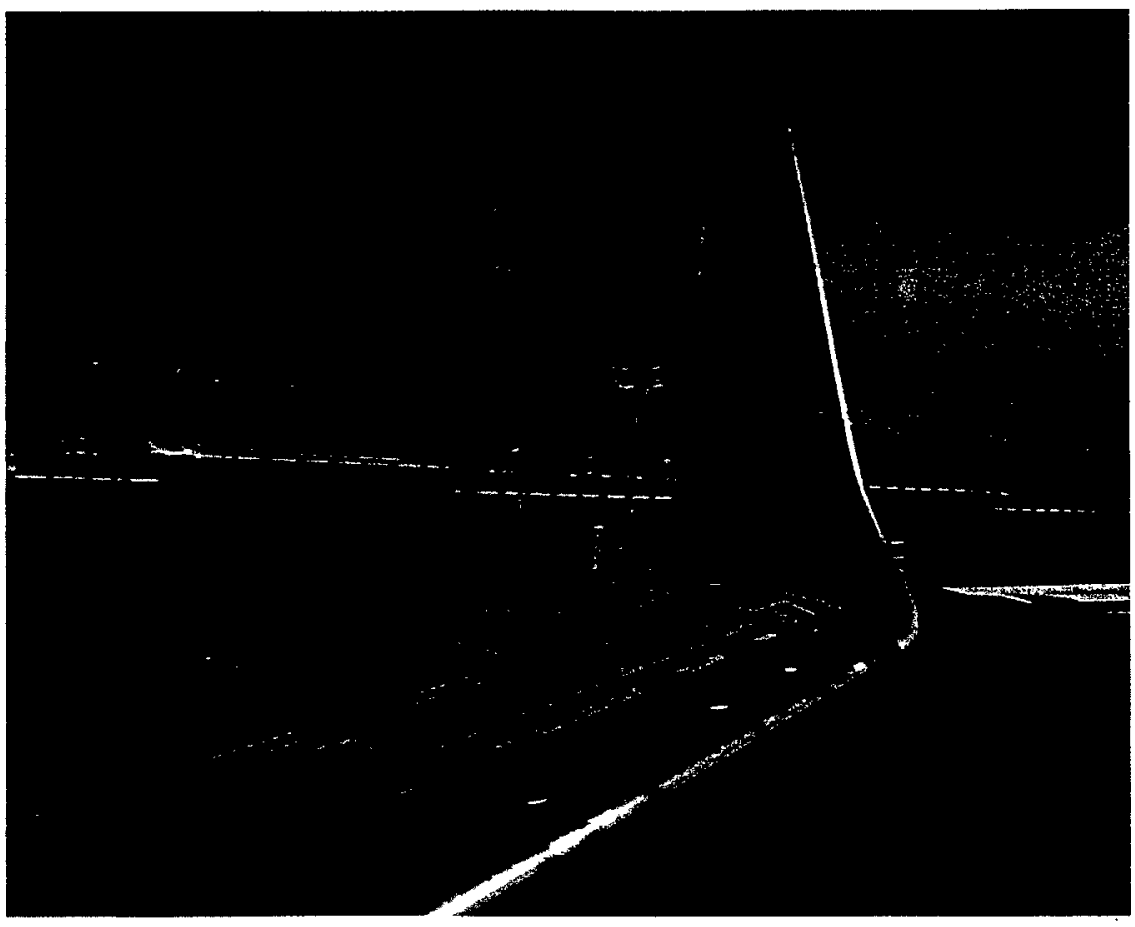

Figure 6.9. Photograph of final installation on the BBJ forward slat.

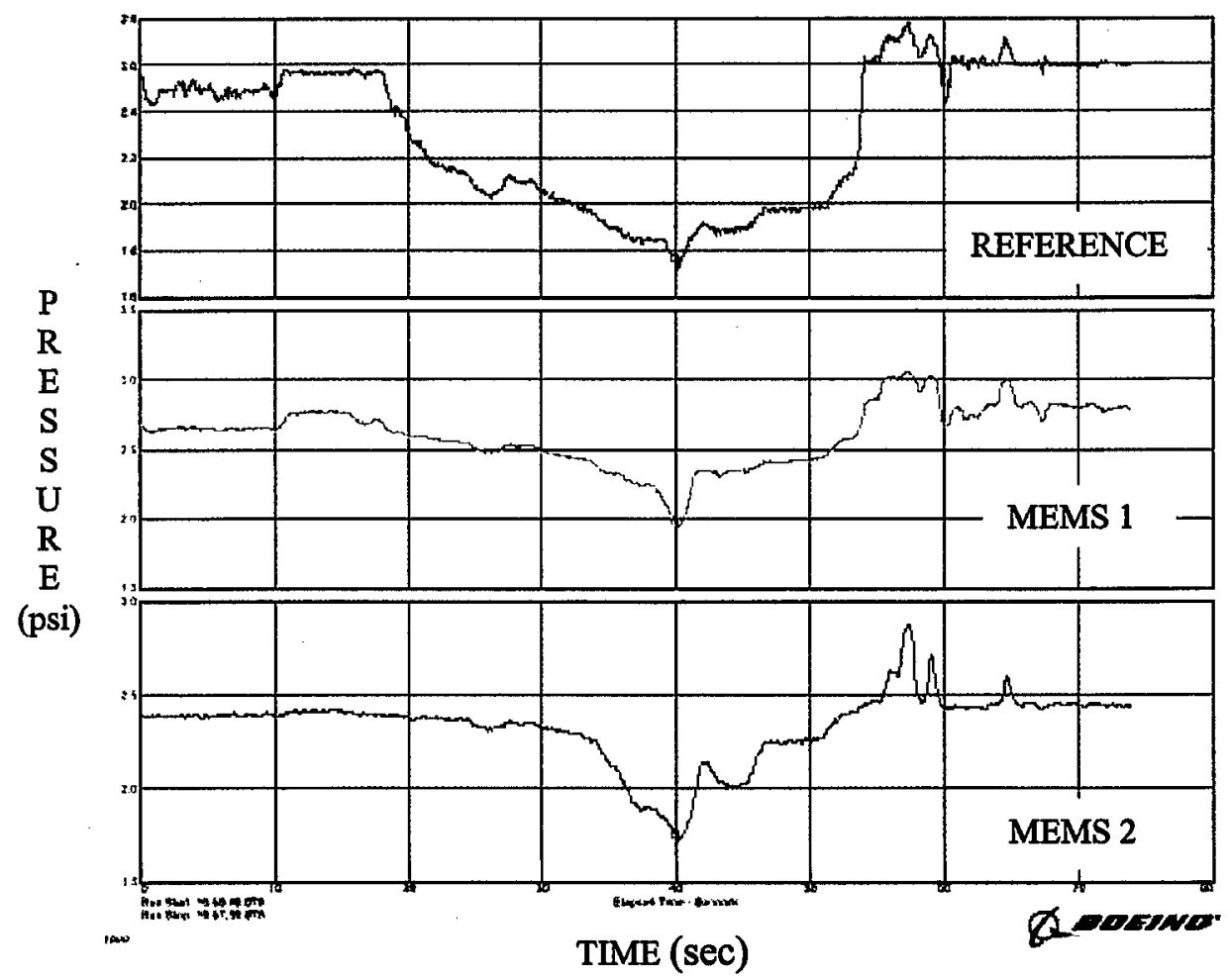

Figure 6.10. Pressure measurements as a function of time for a "wind-up turn" of the BBJ. 
The prototype MCM sensor data tracked well with pressure data recorded from the flush reference pressure ports, also located at $35 \%$ of the slat chord. We believe with a high degree of confidence that the prototype sensor data was as good as the data from the $757 \mathrm{flight}$ test, even though we could not compare them directly due to the fact that they were not adjacent to each other. Some flight data are shown in Figures 6.10 and 6.11. What is important is they showed the exact same trend as the conventional system.

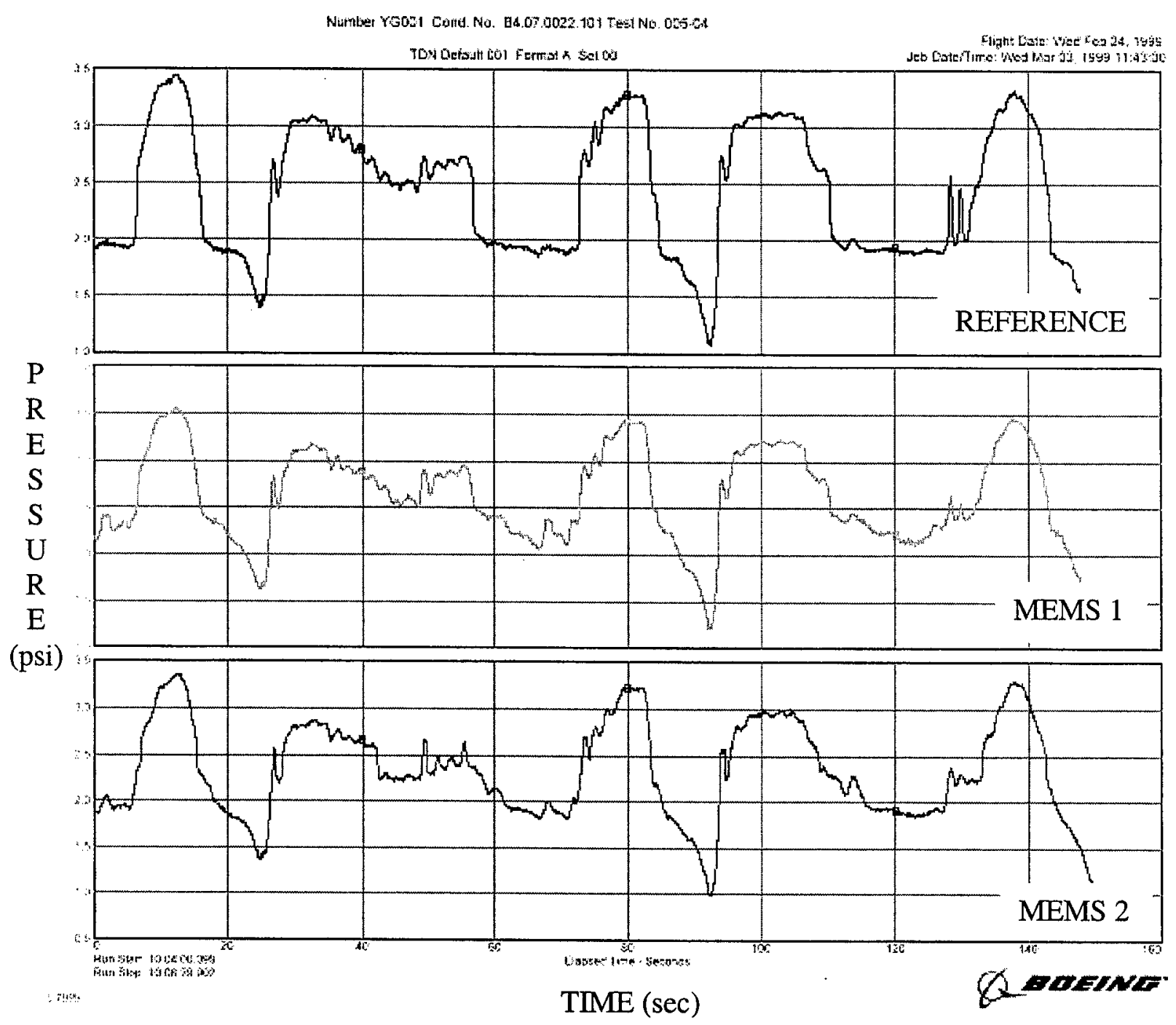

Figure 6.11. Pressure measurements as a function of time for a "roller-coaster" maneuver.

In all the flight segments, pre and post-flight calibrations were performed. This was done both in the lab, where accurate measurements could be done in a controlled environment, and in the field. Table 6.1 shows pre-flight and post-flight pressures measured on the BBJ, while the airplane was parked on the runway at Boeing Field. What are listed are the various instruments on the airplane, that are set to measure pressure, and their readings compared with the MEMS sensors. The data show an agreement between all the sensors, which is within the desired $0.1 \%$ accuracy envelope. The slight variances between the devices are most likely due to the 
difference in their locations and the presence of a light ambient breeze, which could result in small, localized pressure variations.

Table 6.1. Pre-flight and post-flight average pressures measured on the BBJ airplane

\begin{tabular}{|c|c|c|c|}
\hline Pressure (psi) & Pre-flight & Post-flight & Delta \\
\hline & & & \\
\hline Pitot Total Pressure & 14.086 & 14.056 & -0.030 \\
\hline Reference Manifold & 14.055 & 14.023 & -0.032 \\
\hline MEMS 1 & 14.117 & 14.072 & -0.045 \\
\hline MEMS 2 & 14.091 & 14.054 & -0.037 \\
\hline A/P Static & 14.085 & 14.053 & -0.032 \\
\hline Slat 1 belt @ .35 & 14.055 & 14.016 & -0.039 \\
\hline
\end{tabular}

It is well known that for a transonic aircraft, the pressure at the leading edge of the wing is a function of location. In Figure 6.12, we plot the local speed along the slat cord of the BBJ wing during cruise conditions. The speed was calculated using Computational Fluid Dynamics. As one can clearly see, the local speed at the location where the MEMS sensors were placed (marked by the line and $x / c=0.35$ ) is supersonic, with a Mach Number of about 1.3. This was another validation of the pressure belt concept, at a range of speeds more typically encountered by military fighter aircraft.

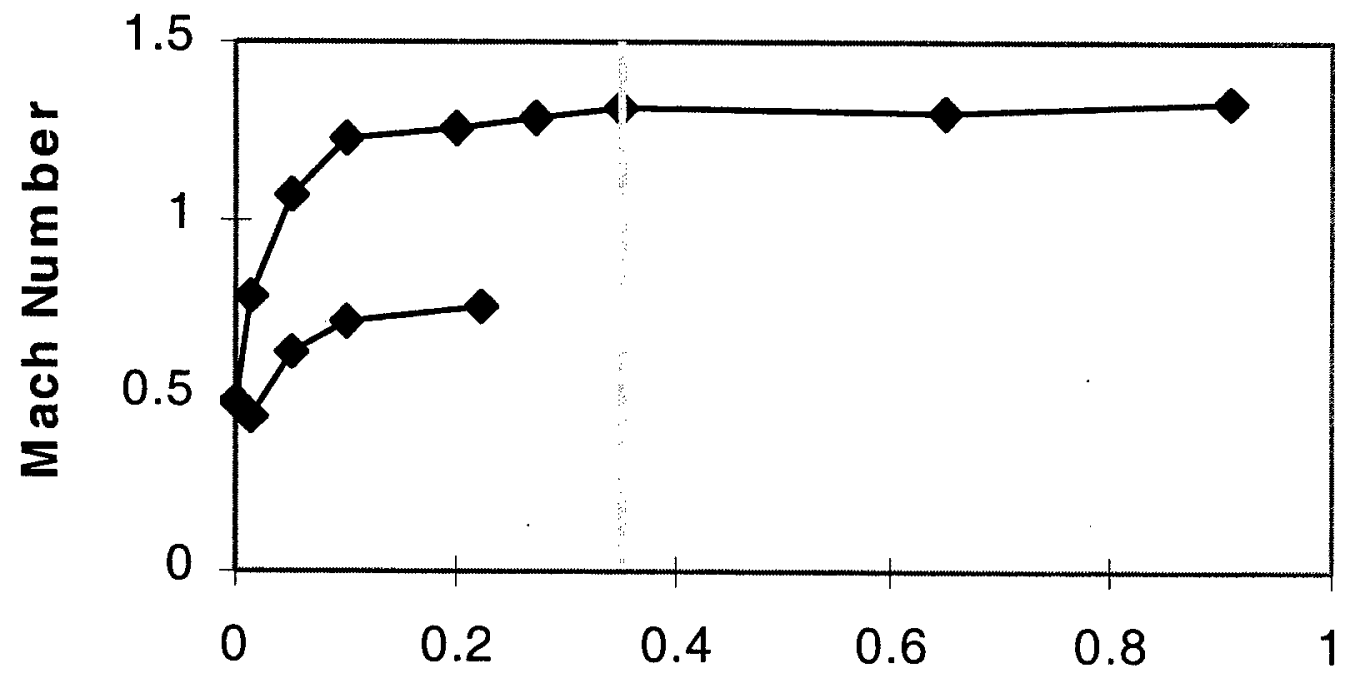

\section{Slat X/C}

Figure 6.12. Calculated air speed as a function of location (cord fraction) on the BBJ wing.

One of the items we also wanted to evaluate was the long term reliability of the materials and processes used on the pressure belt modules in the flight environment. To achieve this goal, we attached a single prototype MCM to an airplane, which was undergoing flight tests, and left it there for several months. Since we were aware of the failure that had taken place on the BBJ, this module had a protective shield over the MEMS sensor. The MCM used was the analog module, on which we had collected a lot of data in the lab. It was calibrated over the temperature range of -48 to $48^{\circ} \mathrm{C}$ and pressure range of 0.5 to $15.0 \mathrm{psia}$. The calibration date 
was $09 / 18 / 1999$. The prototype smart sensor module was installed on $12 / 21 / 1999$ on a $767-400$ undergoing Basic Certification Testing. Installation was located near the aircraft center of gravity, on the underside of the fuselage. The MCM had no electrical wiring connected to the airplane data acquisition system, thus it was not powered during these tests and there was no signal monitoring over the test duration. A photograph of the 767 aircraft taken at Boeing field on a stormy day, just prior to takeoff, and a close-up of the module attached to its belly are shown in Figure 6.13.

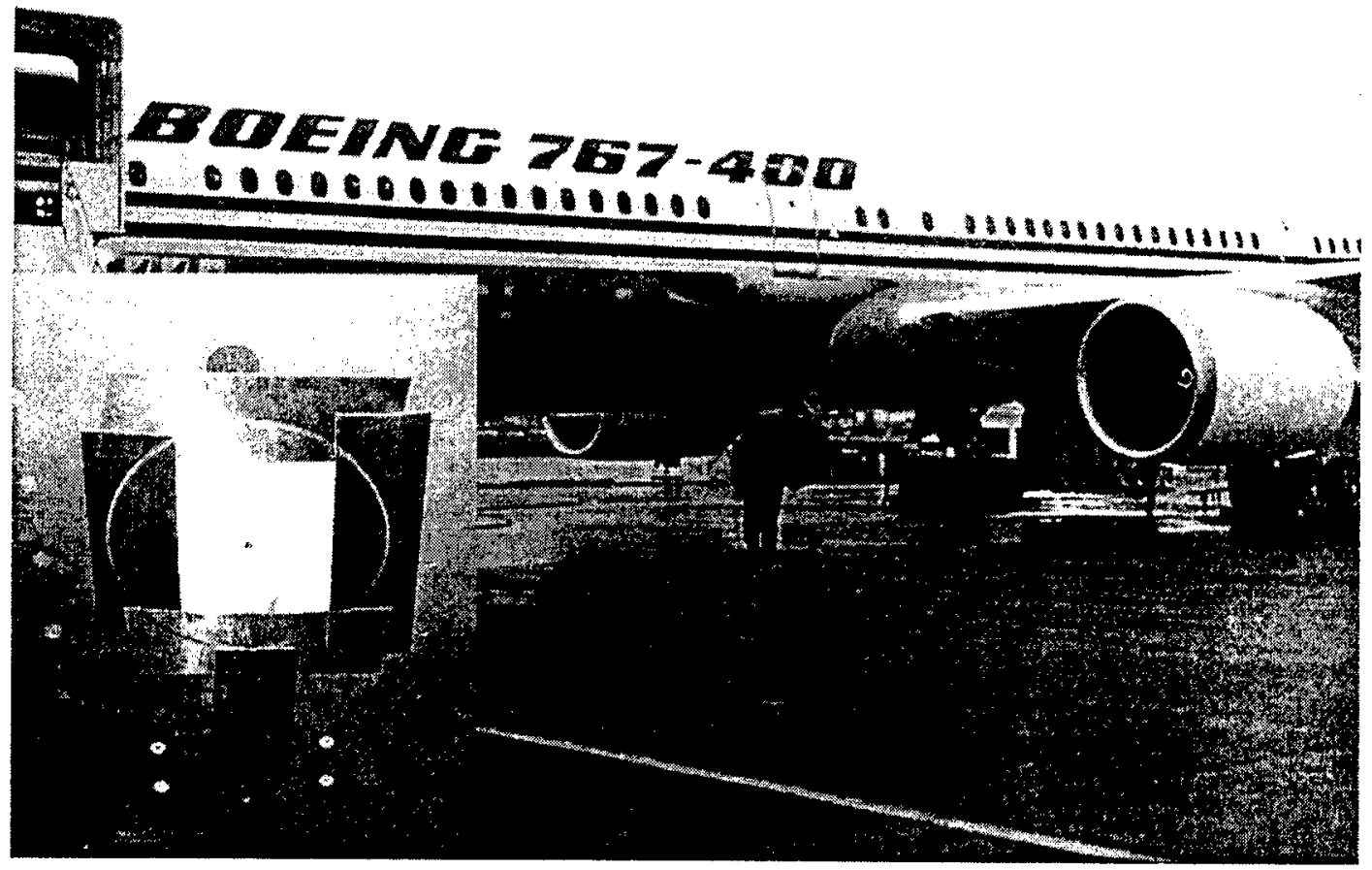

Figure 6.13. Photograph of the 767-400 aircraft and a close-up of the module installation.

The overall purpose of this test was to verify that a module could survive under rather harsh conditions in the field for extended periods of time. The total test duration was over 3 months. This period of time is significantly longer that the expected flight loads testing, which typically is concluded in about two weeks. The total number of flights this module experienced was 59, with multiple takeoffs and landings during each flight. The module was airborne for a total of 111 hours and 46 minutes. Some of the aircraft test conditions included brake testing, crosswind landings, high altitude stalls, wind up turns, dutch rolls, and multiple aggressive hardover airplane maneuvers common in basic certification test programs. 
The module was removed from the 767-400 aircraft on 03/31/2000 and a post calibration was conducted on $05 / 12 / 2000$. The MCM was subjected to an automated test that applied controlled temperature and pressure in the same sequence as the test conducted on 9/18/1999. A comparison of the output revealed a deviation in sensitivity from the baseline data. For temperatures equal to or above $10^{\circ} \mathrm{C}$, the calibration data was within $0.5 \%$ of the full scale output of the baseline test. This was a welcome result, considering that this module was not designed for long duration environmental exposure. The results are shown in Figure 6.14. However, at temperatures equal to or below $0^{\circ} \mathrm{C}$ the output sensitivity decreased with decreasing temperature, with deviations which were several percentage points. This result is shown in Figure 6.15. This effect had not been observed during previous flight tests of the prototype MCM. We tried to identify the cause of this departure from the calibration curve. One possible source of error was in the memory chip, where the calibration coefficients reside. However, the calibration factors were reloaded and the observed behavior persisted. We did observe that two pads on the segment polyimide had lifted off. However, upon closer examination this was eliminated as the source of this behavior. Since this specific module was built early in the program, it is possible that the observed behavior was due to an, as yet, unknown manufacturing defect.

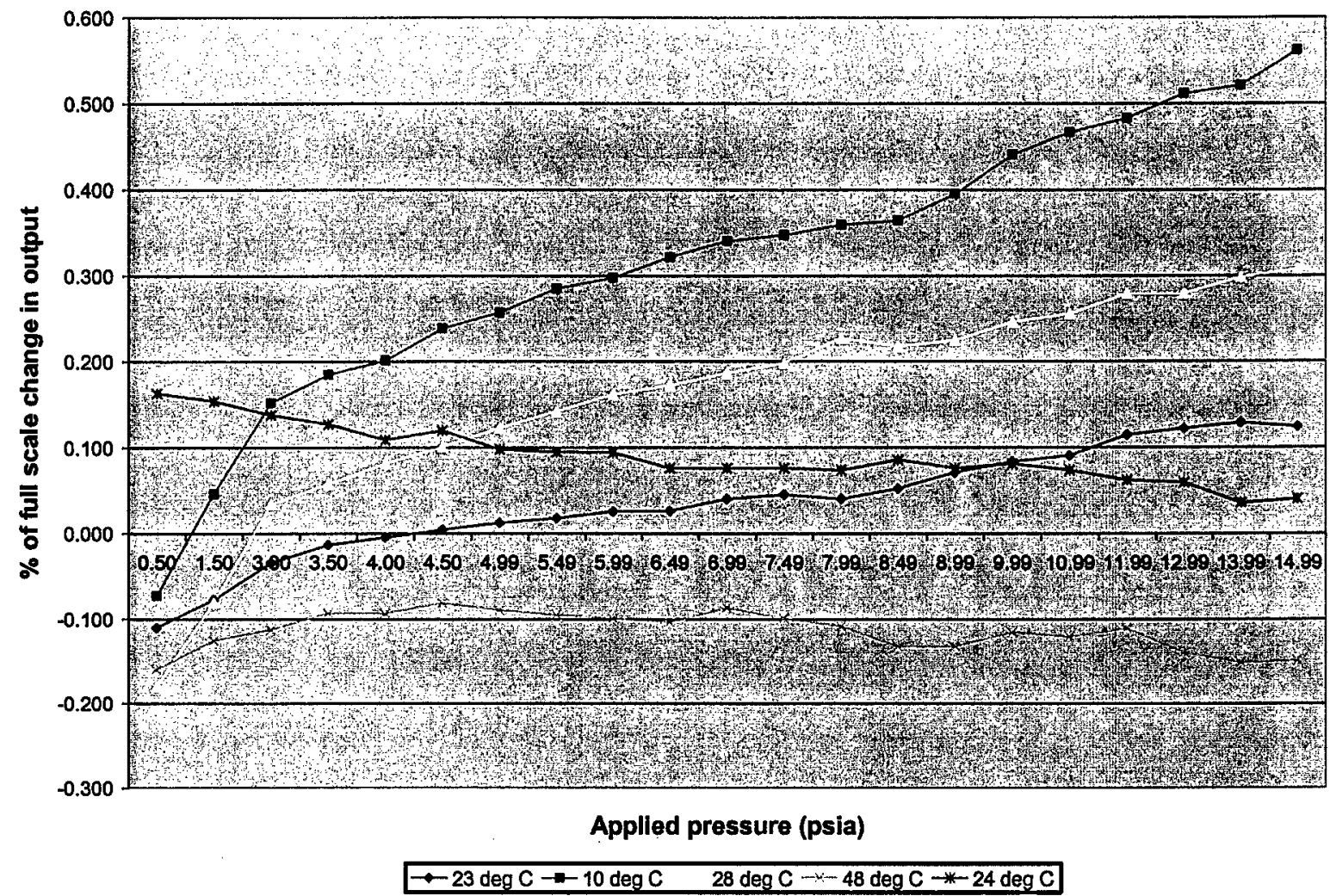

Figure 6.14. Calibration curve comparison equal to or above $10^{\circ} \mathrm{C}$ after long duration test. 


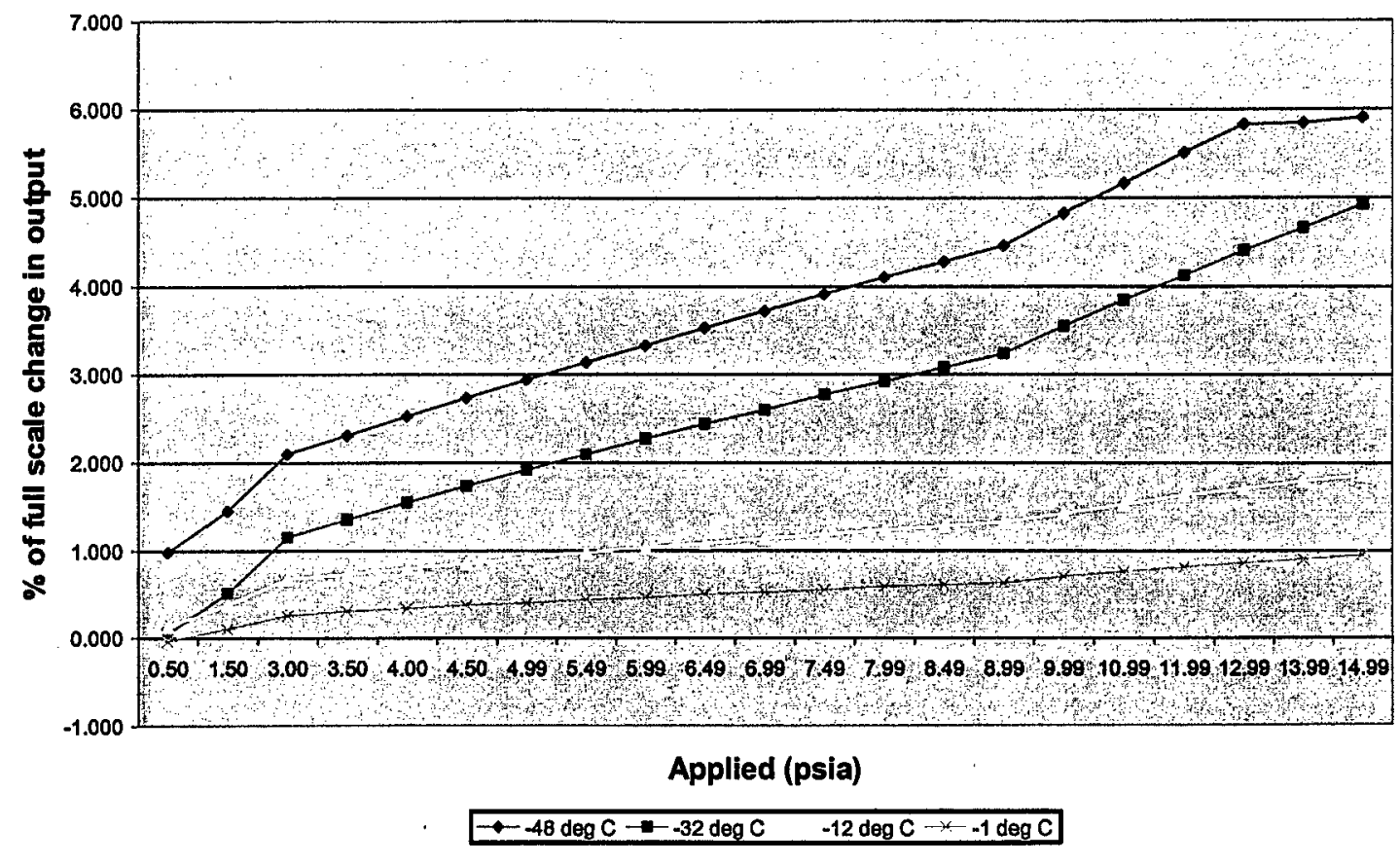

Figure 6.15. Calibration curve comparison equal to or below $0^{\circ} \mathrm{C}$ after long duration test.

As mentioned previously, it is generally prohibitively expensive to schedule a flight test on an aircraft strictly for instrumentation improvements. This is true for both commercial and military aircraft. Tests are most often performed by "piggy-backing" on previously scheduled flight test activities. One such opportunity presented itself during the testing schedule of the F-18E airplane. The F-18E is a new derivative of the F-18 interceptor and was undergoing flight testing for the U.S. Navy at the Patuxent River Naval Air Warfare Center, MD during the last quarter of 2000. It was important for the pressure belt program to validate the concept on a military aircraft, in accordance with the DARPA mission. To this end, we asked the Boeing program manager and the Navy instrumentation organization for permission to fly our prototype hardware on their F-18E3 aircraft. The analog version of the pressure belt was chosen for these tests. There were several reasons for this choice. First, the digital version was still undergoing laboratory tests, so it was not yet available. Second, the analog version had already been flown on commercial aircraft, so we knew it was a viable design. Finally, the analog modules could readily be interfaced to existing instrumentation on the aircraft. However, there was still one more issue that needed to be addressed. The flight envelope of a military airplane is quite different than those of commercial airplanes. It was necessary to convince the Chief Engineer of the F-18E program that our hardware had a fair chance of survival on his airplane. To get some more assurance on this issue, we were requested to do a vibration test. The vibration spectrum used in these tests is shown in Figure 6.16. The calibration curve of an analog prototype module was checked before and after the random vibration testing (15-min per axis in all three axes) and found to be very consistent. These data are shown in Figure 6.17. As one can see, there is little change in the sensor calibration data. 


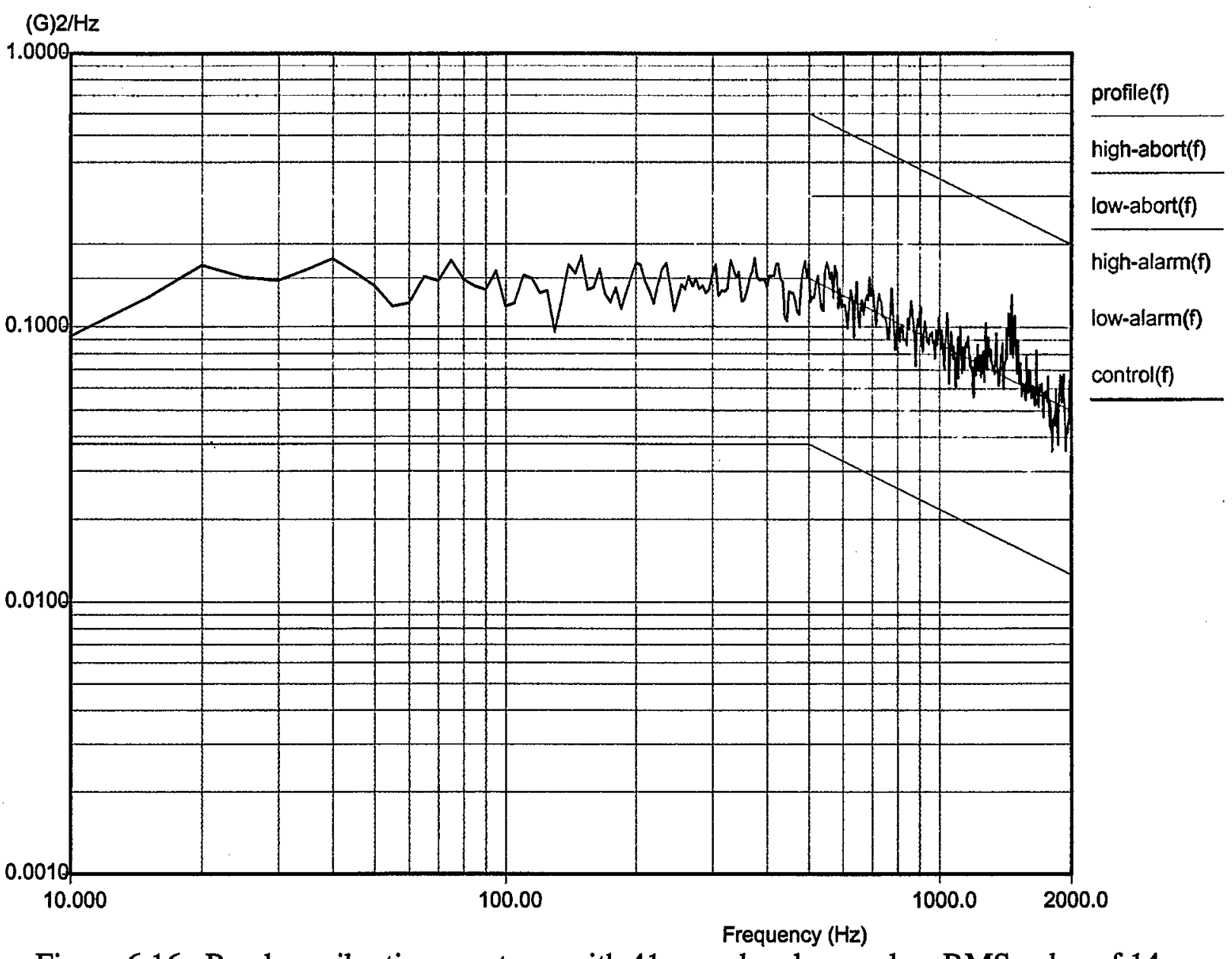

Figure 6.16. Random vibration spectrum with 41-g peak values and an RMS value of $14-\mathrm{g}$.

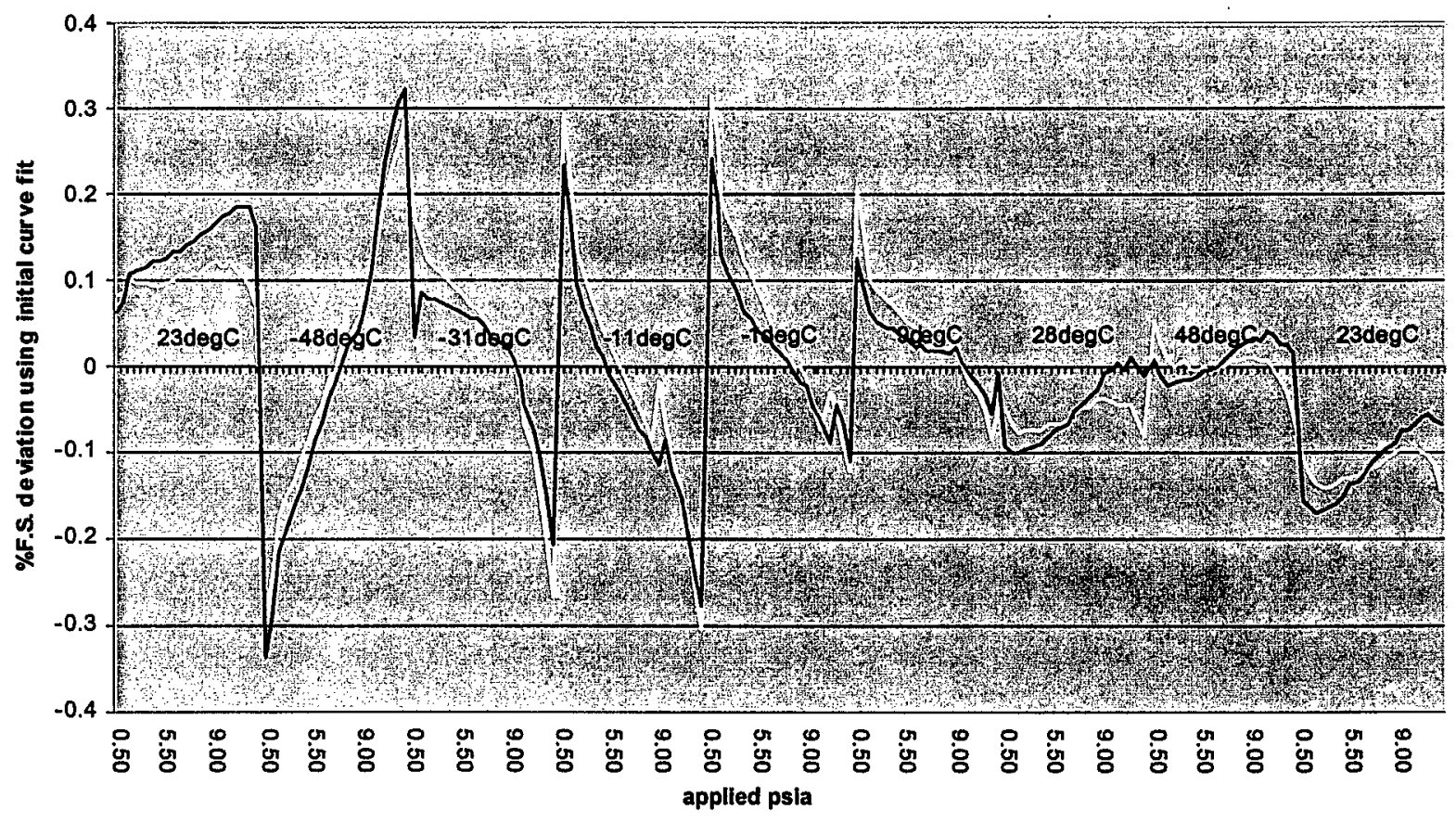

Figure 6.17. Sensor calibration curves before and after 3-axes vibration testing ( $15 \mathrm{~min} / \mathrm{axis})$. 
The prototype analog pressure belt modules were installed on the F-18E3 aircraft during the latter part of September 2000 and were flown during October and November 2000. The F-18E3 airplane on which the hardware was installed is shown in Figure 6.18.

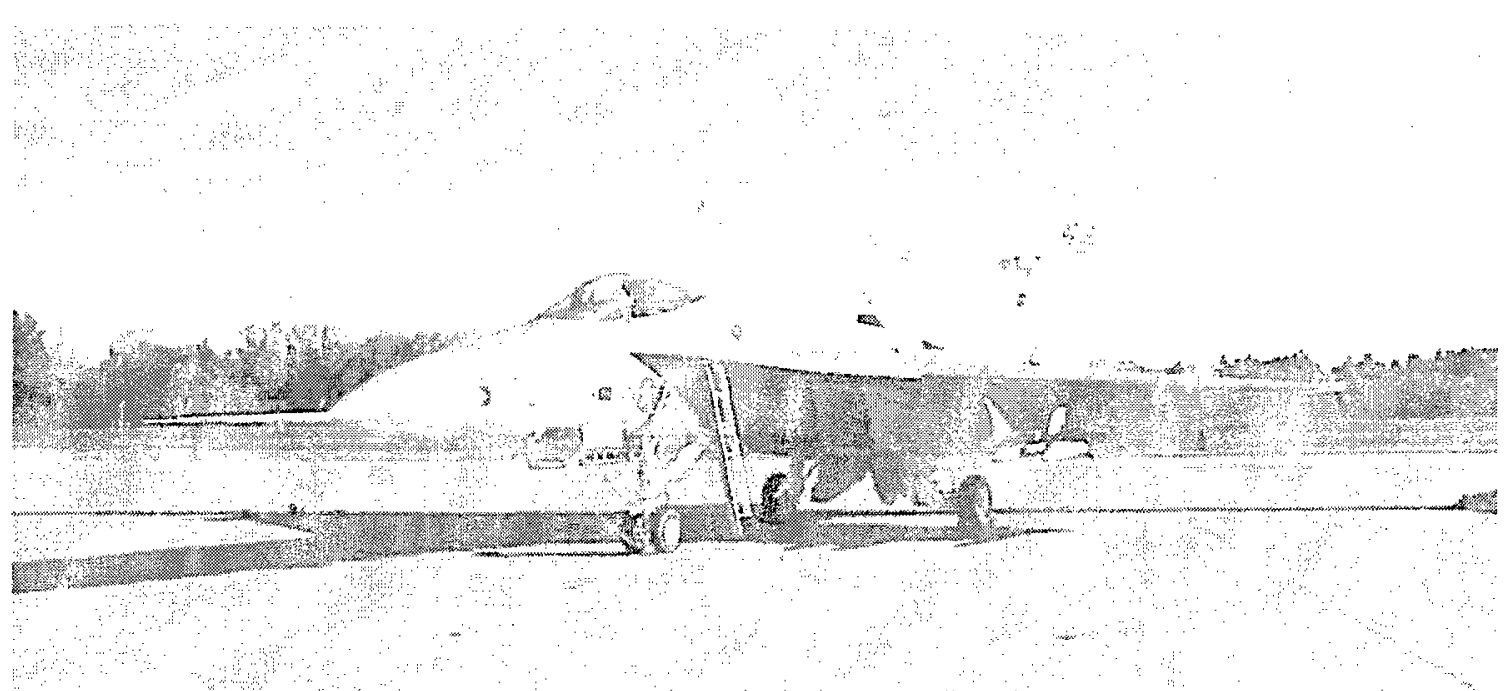

Figure 6.18. The Navy F-18E3 undergoing flight tests at NAWC at Patuxent River.

The pressure belt was attached to one of the airplane pylons. This location provided easy access to the instrumentation bay. This is shown in Figure 6.19 in two different configurations of the pylons. The arrows point at the location of the pressure belt (covered by a fairing) on the inside pylon for two payload configurations. Figure 6.20 shows a close-up of the installation. The reference sensor is located just below the sensor belt.

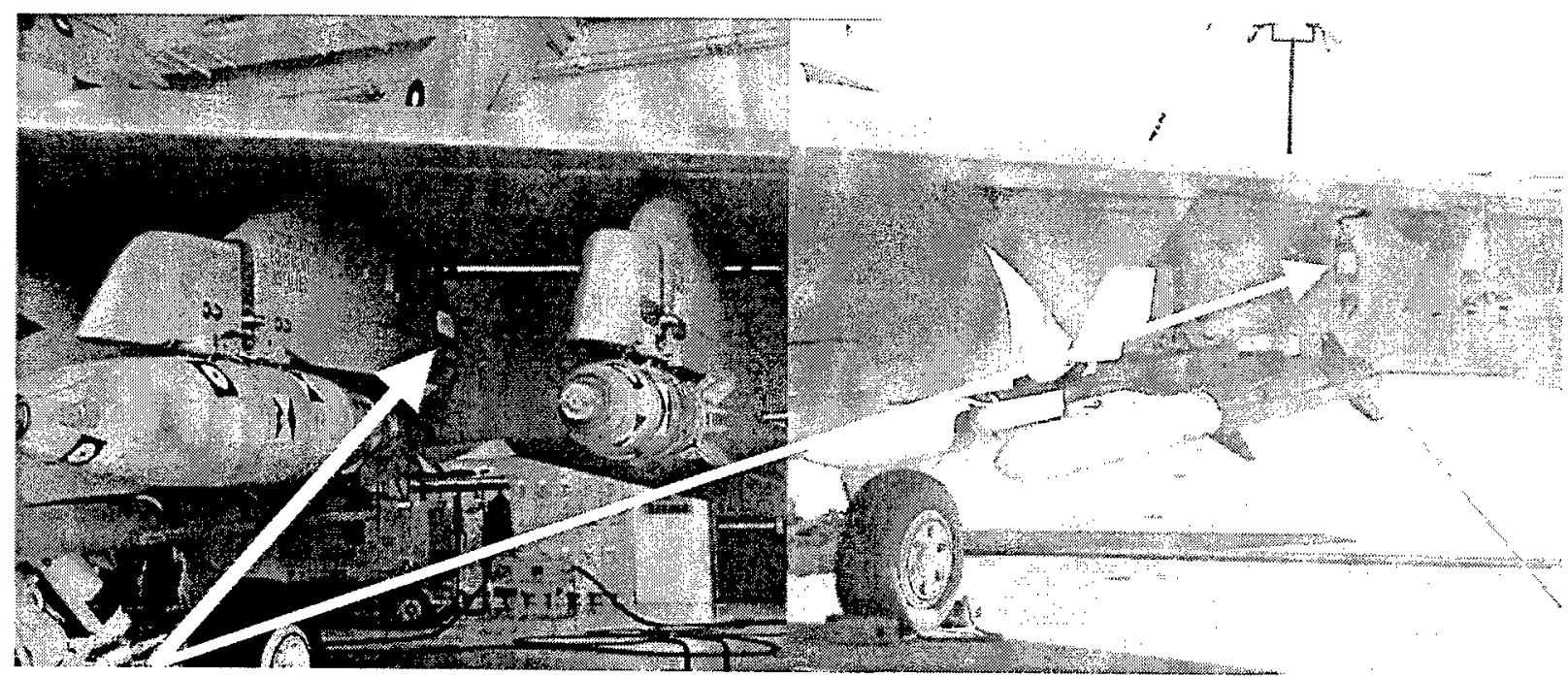

Figure 6.19. Installation of the pressure belt on a F-18E3 at Patuxent River Naval Station. 


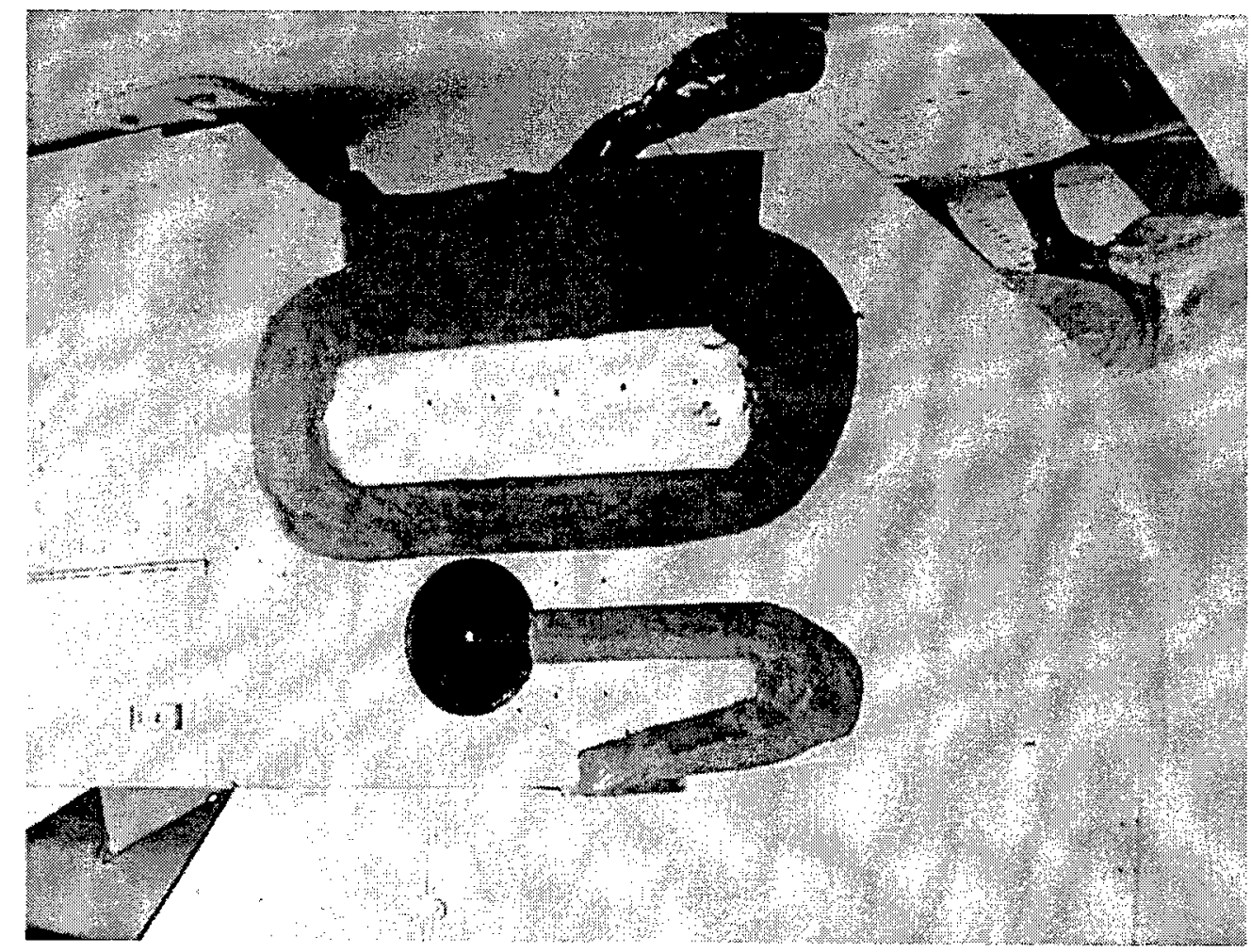

Figure 6.20. A close-up of the pressure belt installation on the F-18E3.

Some of the flight conditions the F-18E3 experienced during the period our pressure belt was installed, are given in Table 6.2. Figures 6.21 and 6.22 show some flight test data from the F-18E3 airplane.

Table 6.2. F-18E3 flight conditions. RPO = Rolling Pull Out, SSPD = Steady State Push Down, SSPU = Steady State Push Up, WUT = Wind Up Turn

\begin{tabular}{|c|c|c|c|c|c|c|c|c|c|c|}
\hline FLT \# & Flight Max & Max Min & \multicolumn{4}{c|}{ Maneuvers } \\
& Time & Alt & G & G & RPO SSPD SSPU WUT & 180 Roll 360 Roll \\
\hline 458 & 2.6 & $20 \mathrm{~K}$ & 7.1 & -3 & $\mathrm{X}$ & $\mathrm{X}$ & $\mathrm{X}$ & $\mathrm{X}$ & & $\mathrm{X}$ \\
\hline 459 & 0.6 & $10 \mathrm{~K}$ & 7.5 & -1 & $\mathrm{X}$ & $\mathrm{X}$ & $\mathrm{X}$ & $\mathrm{X}$ & & \\
\hline $460-462$ & 2.2 & $20 \mathrm{~K}$ & 7.5 & -1 & $\mathrm{X}$ & $\mathrm{X}$ & $\mathrm{X}$ & $\mathrm{X}$ & $\mathrm{X}$ & \\
\hline $463-464$ & 1.7 & $20 \mathrm{~K}$ & 6 & -1 & $\mathrm{X}$ & $\mathrm{X}$ & & $\mathrm{X}$ & $\mathrm{X}$ & $\mathrm{X}$ \\
\hline 465 & 2.4 & $25 \mathrm{~K}$ & 6 & -1 & $\mathrm{X}$ & $\mathrm{X}$ & & $\mathrm{X}$ & $\mathrm{X}$ & $\mathrm{X}$ \\
\hline 466 & 2.5 & $25 \mathrm{~K}$ & 6 & -1 & & $\mathrm{X}$ & & $\mathrm{X}$ & $\mathrm{X}$ & $\mathrm{X}$ \\
\hline 467 & 0.8 & $15 \mathrm{~K}$ & 6 & -1 & $\mathrm{X}$ & $\mathrm{X}$ & & $\mathrm{X}$ & & \\
\hline 468 & 0.9 & $15 \mathrm{~K}$ & 6 & -1 & & $\mathrm{X}$ & & $\mathrm{X}$ & $\mathrm{X}$ & $\mathrm{X}$ \\
\hline 469 & 2.3 & $36 \mathrm{~K}$ & 6 & -1 & $\mathrm{X}$ & $\mathrm{X}$ & & $\mathrm{X}$ & $\mathrm{X}$ & $\mathrm{X}$ \\
\hline 470 & 2.4 & $10 \mathrm{~K}$ & 6 & -1 & $\mathrm{X}$ & $\mathrm{X}$ & & $\mathrm{X}$ & $\mathrm{X}$ & \\
\hline
\end{tabular}




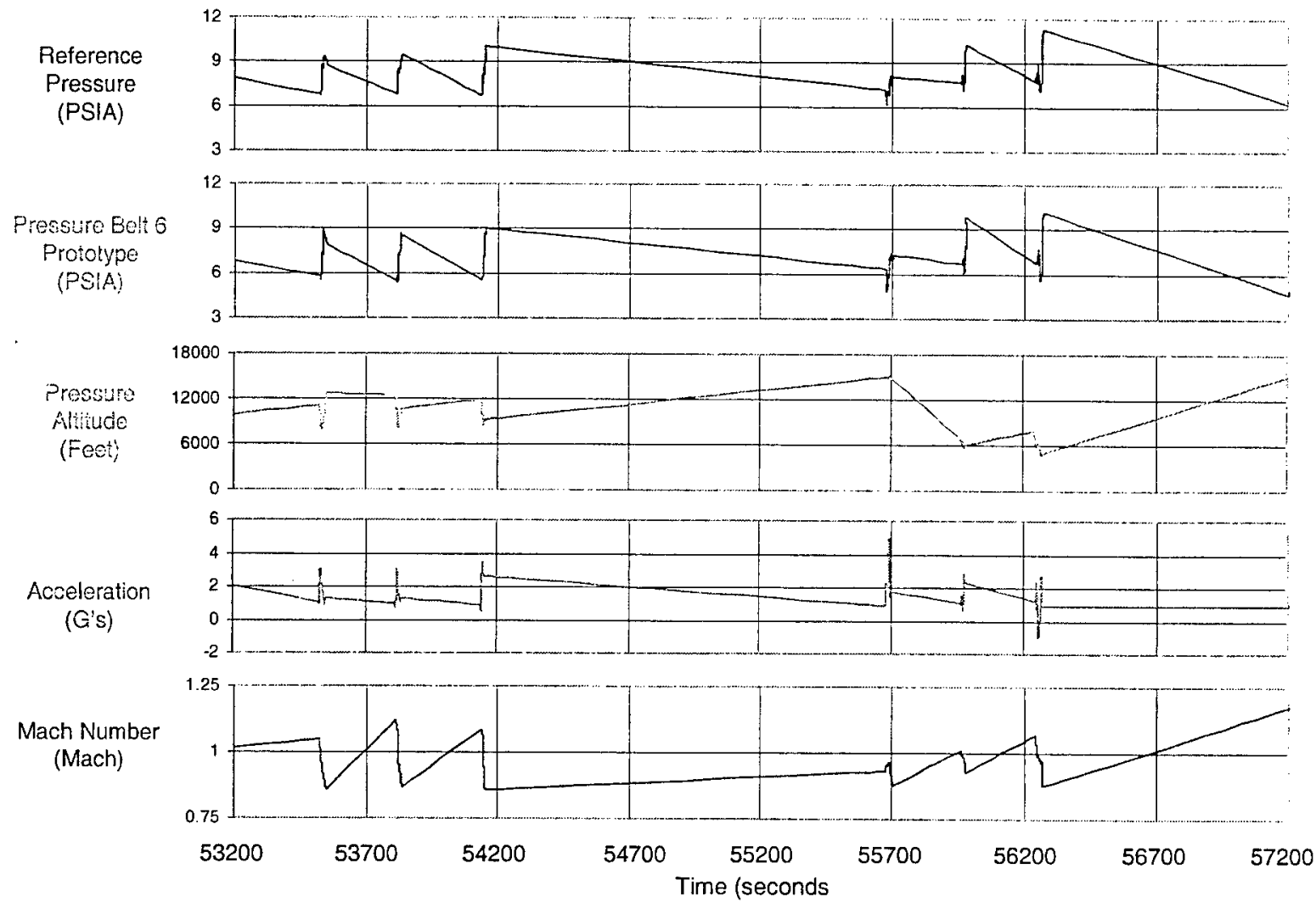

Figure 6.21. Pressure measurement of the MEMS sensor compared to a reference pressure, altitude, acceleration and Mach number.

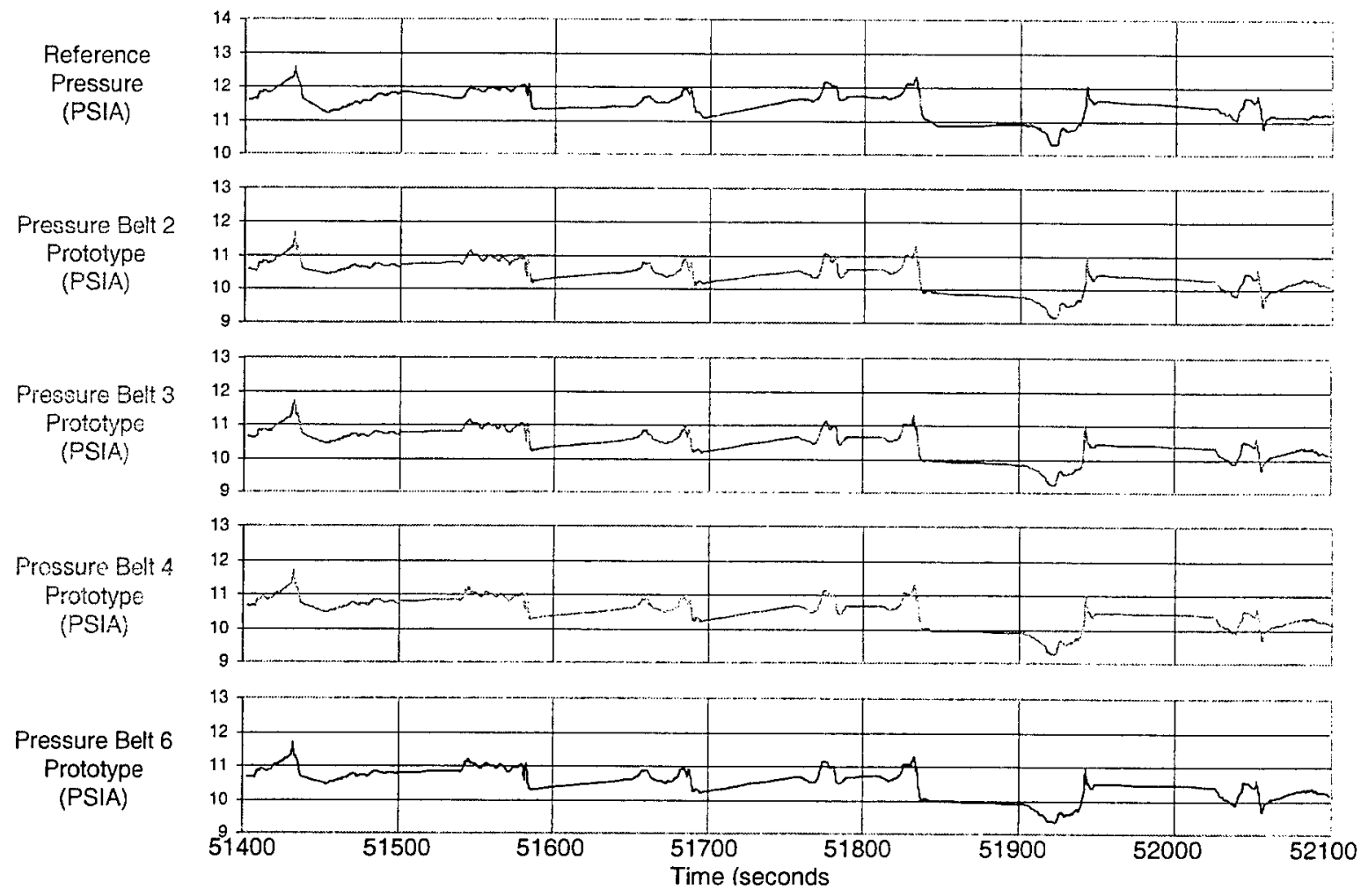

Figure 6.22. A comparison of the reference pressure to the four MEMS channels available. 
Figure 6.23 is an expanded portion of the data shown in Figure 6.22. A close comparison of the reference sensor (Endevco 8515 MEMS sensor) and one of our MEMS sensor readings reveals that even though we are using essentially the same sensor, our package has much better dynamic response than the conventionally packaged part. As one can see, the "peaks" and "valleys" are more filtered in the reference pressure readings. This justifies our decision to go with an unpackaged part in our pressure belt. The offset between the absolute value measured by the two sensors is a calibration artifact.

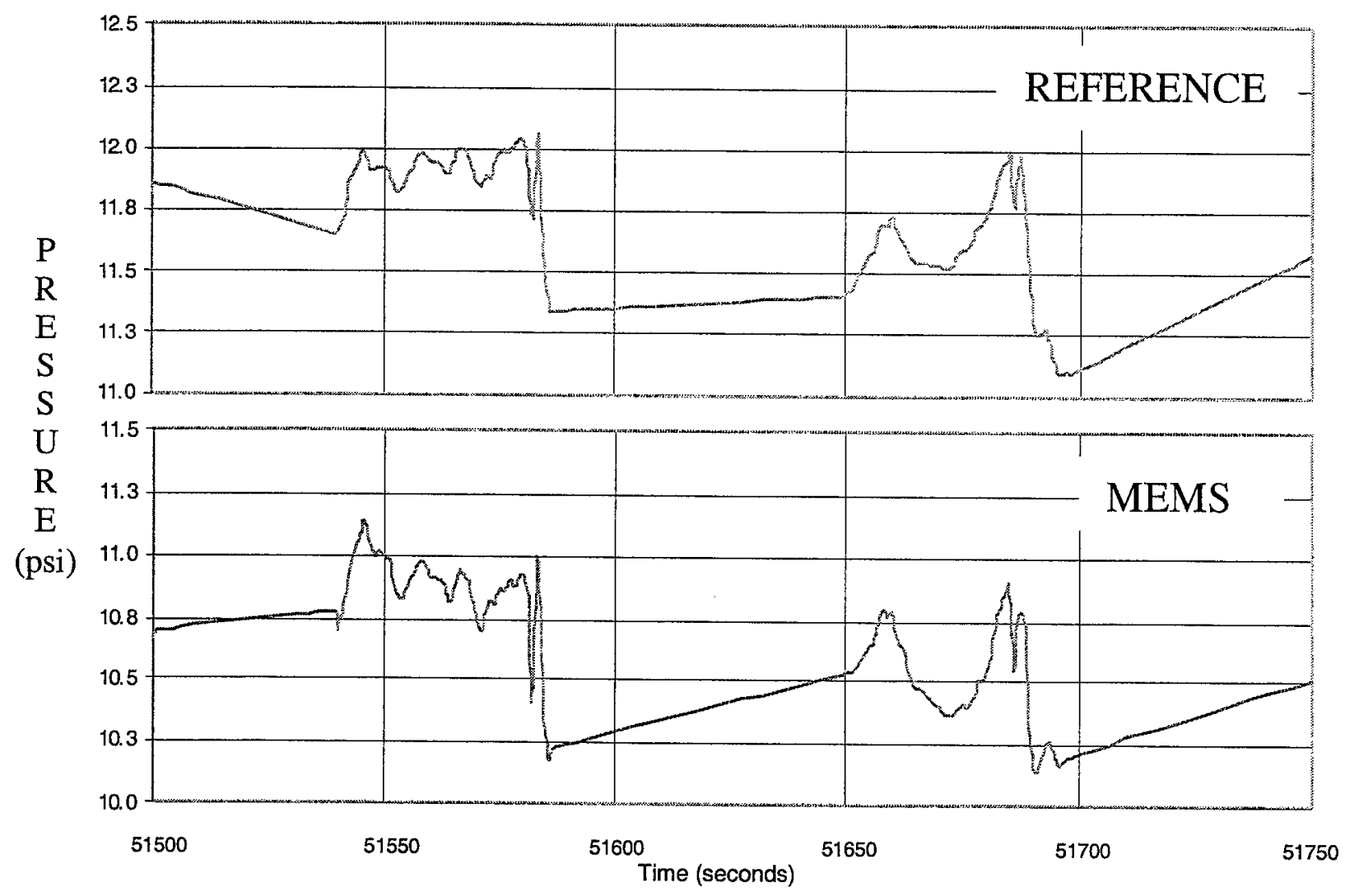

Figure 6.23. An expanded portion of the comparison of the reference pressure to one of the MEMS sensors. 


\subsection{SYSTEM INTEGRATION AND DEPLOYMENT ACTIVITIES}

The Pressure Belt development work was supported by both DARPA and The Boeing Company, through internal funding. The primary objectives of the Boeing Smart Sensor activity, which includes the pressure belt as one of its projects, are the development of standard smart sensors and a corresponding sensor data network for connecting sensors to data acquisition systems for aerospace applications. These activities relate to all three Boeing business units, Military Aircraft and Missiles, Commercial Airplanes, and Space Systems and potentially will benefit a number of future Government programs. The payoff in using this technology is the standardization and simplification of the interface between various sensors and their corresponding electronics, and the capability to field large numbers of sensors with very few interconnections. These benefits translate into significant cost savings for both flight testing and vehicle health monitoring, in addition to flow-time reductions for laboratory systems in the area of:

- Signal conditioning electronics (non-labor dollars).

- Installation of test wiring/cabling (non-labor dollars and labor hours).

The overall strategy in these activities has been:

- To perform technology development within Boeing Phantom Works, which supports all three business units, and develops common solutions applicable across the Boeing Enterprise.

- To supplement Government funding for research and development with Boeing internal funds for applications that are relevant to both.

- To maximize the use of Commercial-off-the-Shelf (COTS) hardware and software. We are accomplishing this with the development of a family of IEEE standards - IEEE 1451.1 through .3 .

- To develop solutions in collaboration with the external supplier/vendor community.

- To license the technology to suppliers of instrumentation for aerospace systems.

The typical problem we are trying to solve is shown in Figure 7.1. A major goal has been to reduce the discrete type of sensor interconnections, such as the ones seen in this photograph, and implement a bus architecture.

The major activities performed, fall into the categories of technology development and application development. In the technology development category, the major tasks were: (1) the development of a Network Compatible Applications Processor and Transducer Bus Controller (NCAP/TBC), and (2) the development of the Transducer Bus and Transducer Bus Interface Module (TBIM), which are components that comprise the interface between smart sensors and the NCAP/TBC. These are developed in compliance with the proposed IEEE P1451.3 standard, and schematically drawn in Figure 7.2. One major remaining task is the establishment of the standard, so that various components are available as standard off-the-shelf items. 


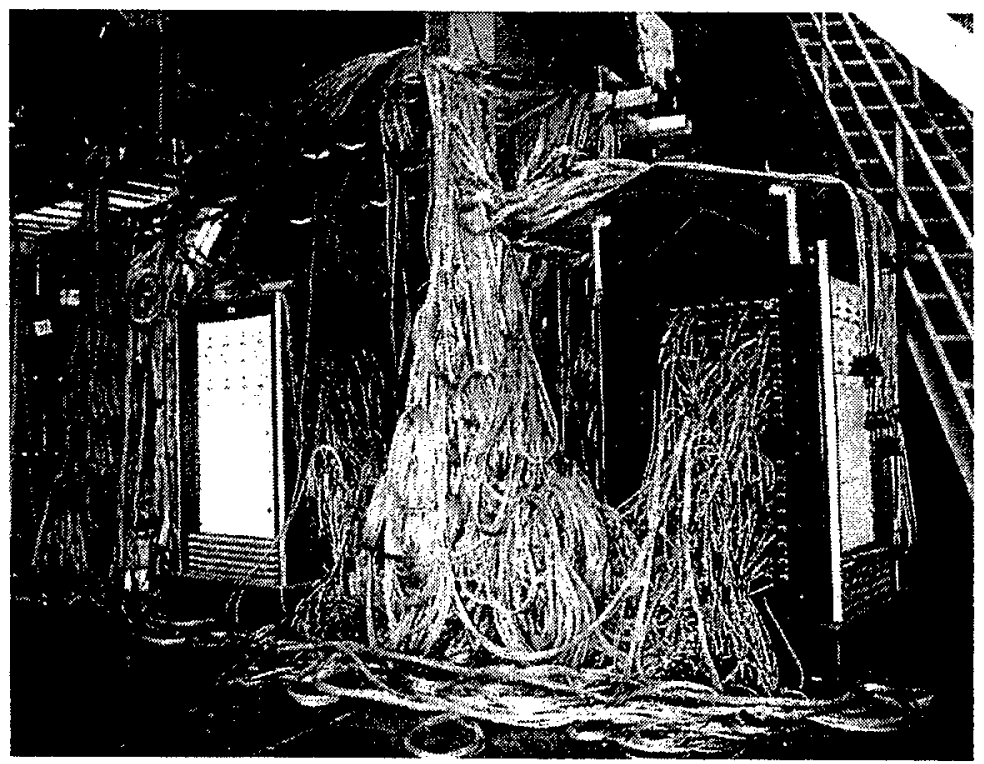

Figure 7.1. A multisensor instrumentation system using conventional point-to-point interconnections.

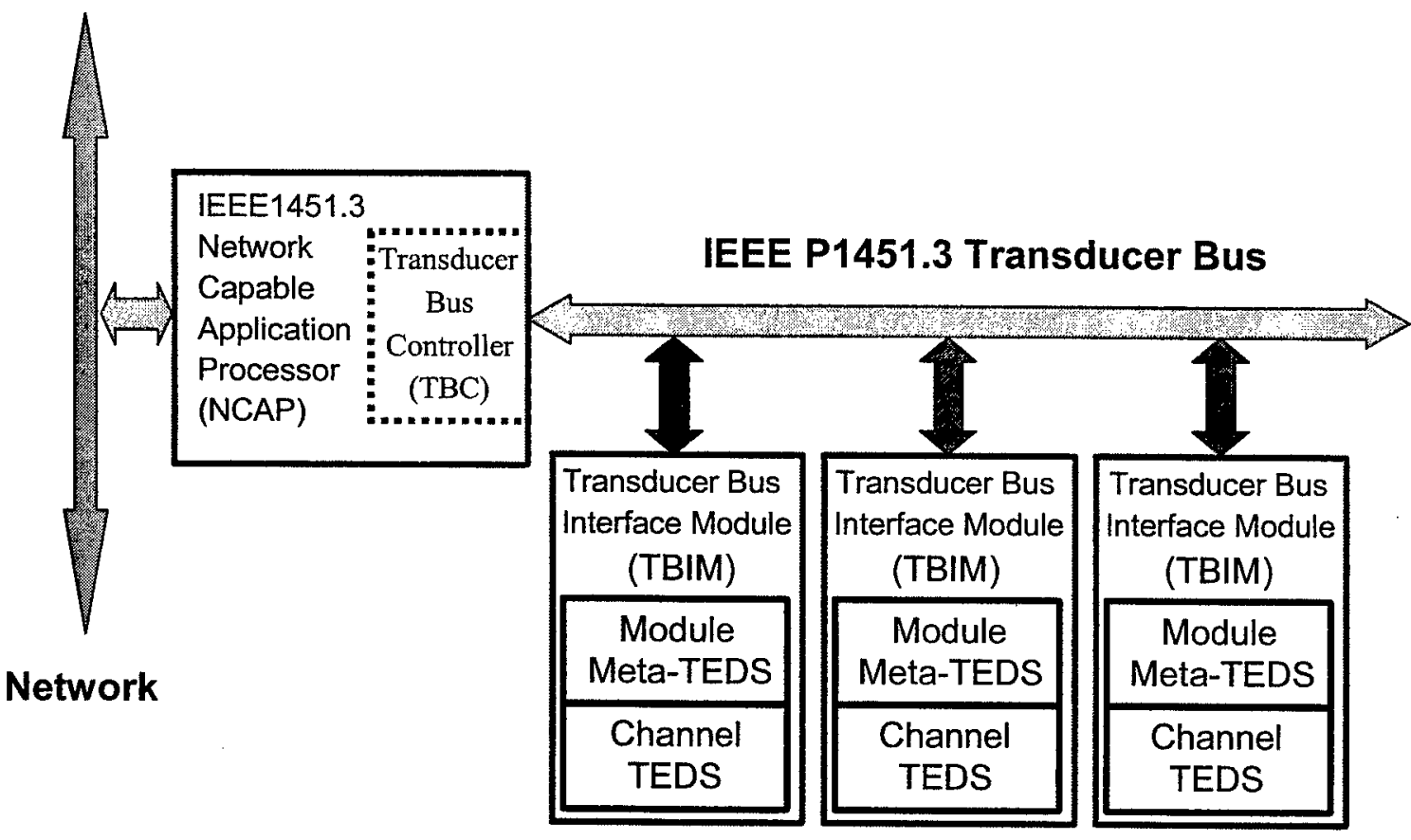

Figure 7.2. The architectural relationship between the NCAP, the TBC and the TBIMs.

We are developing smart sensor capabilities for three applications at this time. These are: (1) a flight test pressure belt and corresponding data system interface, (2) a wind tunnel MEMS pressure sensor module and corresponding data system interface, and (3) a general-purpose TBIM for a variety of sensors such as strain, pressure, load, deflection, temperature, acceleration, and vibration. Much of the technology development for the pressure belt, being funded under this DARPA contract, is also being used for the latter two applications. We are also 
investigating the use of this technology for some more testing on the F-18E \& F aircraft, the Joint Strike Fighter, and the Next Generation Orbiter for NASA.

There is a flight demonstration that is planned by the end of year 2001 of our pressure belt system. It will consist of approximately 11 pressure belt segments consisting of 66 pressure sensors, and an interface to one of our existing flight test data systems. The objective of the demonstration is to show how well the pressure belt performs compared to present day use of electronic pressure scanners connected to tubing in the following areas: measurement lag elimination, data bandwidth increase, less hardware, less instrumentation design, less airplane installation and refurbishment downtime.

A second flight demonstration is scheduled for sometime in 2002. This demonstration will be a much-expanded test compared to the first one. It will consist of approximately 120 pressure belt segments consisting of 720 pressure sensors. The objectives of this demonstration are: (1) to more thoroughly evaluate the performance of the MEMS sensors over the most significant areas of interest of the airplane's flight surfaces; such as the upper and lower wing surfaces, from fuselage to wing tip, and the upper and lower surfaces of the horizontal stabilizer; (2) to evaluate how the sensors perform from leading edge to trailing edge of the flight surfaces; (3) analyze how dense (spacing on the belt segments) the MEMS sensors need to be for the various airplane areas of interest (the current segment design places MEMS sensors at two inch spacing, but not all sensor locations on the segment need to be populated), (4) to study survivability of the sensors when exposed to the flight test environment for the duration of a typical flight loads analysis; and (5) to demonstrate the data bus operation with a network of several loaded-tocapacity transducer buses connected to the data system interface.

With successful completion of these flight demonstrations, we feel that the pressure belt system will become a critical element in flight loads testing of Boeing airplanes.

Finally, Boeing has licensed the pressure belt technology to Endevco Corporation for the manufacturing and commercialization of the pressure belt for flight testing. Endevco, a sensor supplier for many automotive and aerospace instrumentation systems, will make this technology available to all interested parties. 


\subsection{CONCLUSIONS AND RECOMMENDATIONS FOR FUTURE WORK}

There are two basic conclusions as a result of this effort. The first conclusion relates to MEMS technology and its implications for aerospace applications. The second relates to what constitutes the ideal team for technology insertion. These two ideas are discussed below:

1. MEMS sensors are suitable for various aerospace applications, notably flight test. When the project started, what attracted our team towards a MEMS sensor was the fact that it offered high accuracy and it had a small size. One attribute alone was not sufficient for our program needs. Furthermore, the capability that these sensors could be mass-produced and at least in principle, their price could be low was also a motivator for their use. During the course of the program we also became aware of some of the challenges that MEMS devices presented. We found out that if a MEMS device is packaged in a conventional package, which assures its robustness, it also reduces its sensitivity. This tradeoff is not unusual and in fact very common. Thus, the user has to make a conscious choice at the outset. Since one of our objectives was to have the most sensitive device possible, so that we could have very high accuracy in our measurements at the system level, we opted to use the bare device and then ruggedize it to the extent needed for survivability but still maintain a very high degree of accuracy. Similar choices may have to be made by other researchers when using MEMS devices.

2. A team of technologists, users, and commercializers is ideal for technology insertion. One of our observations in this program was that the make-up of a development team has a large impact in whether or not a technology development effort results in a commercial product. There have been many projects funded by DARPA and other Government agencies, which have resulted in new technology developments. However, even though the intention of the developers has been to do technology transfer and ultimately create a new product, this "hand-off" is often incomplete or difficult to accomplish. Partly, this may due to the fact that the commercializers and users of this technology are not part of the development team. This is an important point. Even though it is possible for a development team to work essentially independently during the course of the program, given a set of requirements provided by the users, this is often not sufficient. In our experience, constant feedback and consultation with the users assures a more streamlined activity and clearer focus. It is often assumed that if a technology is promising a commercializer will be willing to step in and "run" with it. This is not always the case, especially if it implies a long product development cycle. Endevco Corporation, which will be the manufacturer of this product, had a lot of suggestions during the course of the program. In essence, what we managed to do was to combine the technology development and product development activities in one. A number of these suggestions were not directly related to the pressure belt application. They were relevant, however, to the viability of this technology for various other applications, which the project had not initially anticipated. This "built-in" versatility made the technology quite attractive and gave them a very strong interest in helping us succeed in our efforts. Furthermore, during the course of the program they made sure that whatever materials, processes, and approaches were used, were suitable for manufacturing and consistent with their internal capabilities and practices. Thus, this combination of team members led to a very successful technology insertion.

In terms of recommendations for future work in this area, we believe that the next generation of smart multisensor systems will integrate MEMS, actuators, and higher level intelligence on the system level to achieve independent action, such as integrated vehicle health management. The 
Boeing-developed technology has already provided the first application of a multisensor system, where as many as several thousand sensors could be deployed. These integration and productization activities were to a large extent funded through Boeing internal funds and Endevco funds, which complemented the activities under the DARPA-sponsored technology development. We believe the establishment of the IEEE P1451.3 standard will facilitate the insertion of such multisensor systems in a variety of commercial and military applications. Furthermore, the integration of the sensors with actuators to provide autonomous control, together with the development of the appropriate software, such as the activities being performed under the DARPA ITO NEST (Networked Embedded Software Technology) program could enable a number of complex functions and applications. 


\section{APPENDIX A: RELATED PUBLICATIONS AND CONFERENCE PRESENTATIONS}

1. Jiali Wu, Randy T. Pike, C. P. Wong, "Novel Bi-layer Conformal Coating for Reliability Without Hermeticity MEMS Encapsulation", IEEE Transactions on CMPT, Part C, vol. 22, No. 3, p. 195 (July 1999).

2. Jiali Wu, Randy T. Pike, C. P. Wong, "Interface-Adhesion-Enhanced Bi-layer Conformal Coating for Avionics Application", 1999 International Symposium on Advanced Packaging Materials, Mar. 14-17, 1999, Chateau Elan, Braselton, Georgia, p. 302-310.

3. Jiali Wu, Randy T. Pike, C. P. Wong, "Novel Bi-layer Conformal Coatings for MEMS Encapsulation---Parylene C Coated Silicone Elastomers", Proceedings of the Pacific Rim/ASME International Intersociety Electronic \& Photonic Packaging Conference, Maui, vol. 26-1, p. 115 (1999).

4. Jiali Wu, Randy T. Pike, C.P. Wong, Namsoo P. Kim, and Minas H. Tanielian, "Evaluation and Characterization of Reliable on-Hermetic Conformal Coatings for Microelectromechanical System (MEMS) Device Encapsulation," IEEE Transactions on Advanced Packaging, Vol.23, No. 4, p. 721, Nov. 2000.

5. N.P. Kim, K. Li, D.J. Kovach, C-P. Chien, M. H. Tanielian, "Smart Sensor Multi-Chip Module with Direct-Chip-Attached Micro-Electro-Mechanical System (MEMS) Devices," Proc. Of $32^{\text {nd }}$ International Symposium on Microelectronics (IMAPS 99), p. 484, Oct. 1999.

6. N. P. Kim, M.J. Holland, M. H. Tanielian and R. Poff, "MEMS Sensor MCM Assembly with TAB Carrier - Pressure Belt for Aircraft Flight Testing," Proc. Of 50th IEEE Electronic Components and Technology Conference, May 2000.

7. N.P.Kim, M.J.Holland, C-P.Chien,M.H.Tanielian, J.Wu, and C.P.Wong, "Aircraft Flight Tests and Reliability Improvements of MEMS Pressure Sensor Assembly," Proc. of Surface Mount Technology Association International (SMTAI 2000) conference, Chicago, IL, Sep. 2000.

8. N.P. Kim, N. Amirgulyan, K. Li, C-P. Chien, and M.H. Tanielian, "Fabrication and Assembly of a Digital Transducer-to-Bus Interface Module Having a Directly Attached MEMS Device," Proc. of $34^{\text {th }}$ International Symposium on Mocroelectronics (IMAPS 2001), Oct. 2001. 


\section{OTHER RELATED ARTICLES}

1. L. Eccles, W. Catlin, M.J. Holland, N. P. Kim, and L. Malchodi, "MEMS Pressure Belt with Sensor Interface and Communication Architecture," Proc. of SPIE Smart Material Conference, Mar. 2001.

2. R. Ramesham, R. Ghaffarian and N.P. Kim, "Reliability Issues of COTS MEMS for Aerospace Applications," Proc. of SPIE International Symposium on Micromachining and Microfabrication, Sep. 22, 1999

\section{PRESENTATIONS}

1. M.H. Tanielian, N.P.Kim, and M.J.Holland, "MEMS Sensor Pressure Belt for Aircraft Flight Testing," at IMAPS ATW (Advanced Technology Workshop) at Washington, D.C. 5-22-2000.

2. M.H. Tanielian, "Flight Loads Surveys Using MEMS Devices," American Physical Society March Meeting, Invited Talk, March 12-16, 2001, Seattle, WA.

3. M.H. Tanielian, "MEMS-Based Aircraft Multisensor System," Military Micro-Systems \& Nano-Systems, June 4-5, 2001, Washington DC 


\title{
Novel Bi-Layer Conformal Coating for Reliability Without Hermeticity MEMS Encapsulation
}

\author{
Jiali Wu, Randy T. Pike, Member, IEEE, and C. P. Wong, Fellow, IEEE
}

\begin{abstract}
A flexible, smooth, and low profile conformal coating was developed to accomplish the encapsulation of a microelectromechanical system (MEMS) device that will be applied to sense the static pressure on aircraft during real flight testing. The encapsulant should be able to protect the MEMS device and the multichip module (MCM) from adverse environmental conditions, i.e., mechanical shock, temperature fluctuation, engine fuel and oil contamination, and moisture/mobile ion permeation. Presently, conventional packaging schemes for electronics cannot satisfy this specific outdoor application, and a new encapsulation combination has been designed in accord with the requirement of reliability without hermeticity (RWOH). A bi-layer structure was selected because of property limitations of a single material. Pliable elastomeric silicones, are typically flexible, water repellant, and abrasion resistant. The silicone encapsulant will be first applied to planarize the MEMS surface and function as durable dielectric insulation, stress-relief, and shock/vibration absorbers over a wide humidity/temperature range. To compensate for the deficiency of silicone on engine fuel/oil contamination, Parylene $\mathrm{C}$ is to be deposited afterward. This bi-layer coating can achieve excellent bulk properties, such as moisture and mobile ion barrier resistance, chemical compatibility, and electrical insulation characteristics. However, the poor adhesion of Parylene $\mathbf{C}$ to silicone greatly restricts its application. To address this problem, silane coupling agents were used as an adhesion promoter. Significant adhesion im provement was achieved by placing an interlayer silane coupling agent to provide interfacial bonding to the silicone elastomeric surface and the Parylene $\mathrm{C}$ film. Furthermore, a possible mechanism of adhesion enhancement will also be presented in this study.
\end{abstract}

Index Terms - Bi-layer conformal coating, micro-electromechanical system (MEMS), multichip module, Parylene $C$, reliability without hermeticity (RWOH), silane coupling agent, silicone elastomer.

\section{INTRODUCTION}

$\mathbf{L}$ ONG-TERM, reliable protection of sensitive circuits and components is becoming more important in many of today's delicate and demanding electronic applications. The need for low profile, flexible, conformal and economical packaging in micro-electromechanical system (MEMS) based modular protection has resulted in a new conformal coating design exhibiting reliability without hermeticity (RWOH). General organic passivation materials such as polyimides, silicones, and epoxies have been used as RWOH encapsulants with reasonable success. However, these organic materials

Manuscript received June 9, 1998; revised June 10, 1999. This work was supported by the Boeing Company and Specialty Coating Systems, Inc.

The authors are with the School of Materials Science and Engineering, Packaging Research Center, Georgia Institute of Technology, Atlanta, GA 30332-0245 USA.

Publisher Item Identifier S 1521-334X(99)08154-9. are generally moisture permeable. Moisture diffusion rates through organic polymeric encapsulants vary with time. The rate depends on the type of material, the material thickness, and the length of diffusion time. Moisture on the surface of the device, in the presence of mobile ions such as $\mathrm{Na}^{+}$, $\mathrm{K}^{+}$, and $\mathrm{Cl}^{-}$, will result in device failure [1]. To ensure RWOH, we propose to combine a low moisture permeable silicone elastomer coating with a thin film of Para-X-xylyene (Parylene $C$ ) to ensure long-term protection. The silicone elastomer is coated as a relatively thick layer (approximately 250 mil), primarily for protection of the delicate circuits and as a planarizing layer for the MEMS device. A thin layer of Parylene C (approximately $15-20 \mu \mathrm{m}$ ) is then deposited on top of the thick silicone elastomer layer. This novel combination provides an outstanding barrier to moisture and mobile ion $\left(\mathrm{Na}^{+}, \mathrm{K}^{+}, \mathrm{Cl}^{-}\right)$permeation and an aerodynamically smooth surface for avionics applications. Furthermore, the low surface energy of Parylene $C$ as a top coating can effectively prevent air borne particles [2] and jet fuel contamination of the underlaying silicone coating.

\section{A. Conformal Coating Materials}

Silicones are typical pliable elastomeric materials, which can function as durable dielectric insulation, as barriers against environmental contamination and as stress relieving shock/vibration absorbers over a very wide humidity/temperature range. In this study, Dow Corning Q4-2817 and Dow Corning Hipec 3-6550 were selected as candidate elastomer, to function explicitly as a top profile planarizing agent.

Parylene is a conformal coating family that has high dielectric strength and excellent chemical resistance [3]. Currently, the Parylene family is composed of four types of commercial products: Parylene N, C, D, and F. Unlike conventional liquid coating, Parylene coatings are applied by vapor deposition in a vacuum chamber at ambient temperature. This unique method of vapor deposition can form $100 \%$ pinhole-free uniform coverage and has the ability to penetrate and coat very complex topographical geometry. Coating thickness with this deposition process is also easily controlled. In this study, Parylene $\mathrm{C}$ is selected as a suitable encapsulating candidate in terms of moisture permeation resistance, chemical resistance, deposition rate and cost consideration [4].

Table I lists the normalized permeability of gases through polymer materials. The permeability of Parylene $\mathrm{C}$ is significantly lower than almost any other engineering plastic. Moisture permeability resistance of Parylene $\mathrm{C}$ is 40 times greater 
TABLE I

Normalized Permeablitty of Gases Through Polymer Materials

\begin{tabular}{c|c|c|c|c|c}
\hline Material & $\mathrm{O}_{2}$ & $\mathrm{~N}_{2}$ & $\mathrm{CO}_{2}$ & $\mathrm{H}_{2}$ & $\mathrm{H}_{2} \mathrm{O}$ \\
\hline Parylene C & 2.8 & 0.4 & 3 & 43 & 0.08 \\
\hline Parylene D & 12 & 1.8 & 5 & 94 & 0.1 \\
\hline Parylene N & 15 & 3 & 84 & 213 & 0.6 \\
\hline HDPE & 73 & 17 & 228 & n.d. & 0.12 \\
\hline PS & 138 & 23 & 400 & n.d. & 3.5 \\
\hline PTFE & 223 & 133 & n.d. & 516 & n.d. \\
\hline LDPE & 140 & 80 & 700 & n.d. & 0.6 \\
\hline PC & 124 & 22 & 827 & n.d. & 1.5 \\
\hline FEP & 295 & 126 & 657 & 381 & 0.16 \\
\hline Silicone & 19000 & n.d. & 118000 & 17000 & 3 \\
\hline
\end{tabular}

TABLE II

Swelling Caused by Organic Solvents at Room Temperature

\begin{tabular}{l|l|c|c|c}
\hline \multicolumn{2}{c|}{ Solvents } & \multicolumn{3}{c}{$\%$ Swelling Parylene } \\
\hline Class & Test Member & $\mathrm{N}$ & $\mathrm{C}$ & $\mathrm{D}$ \\
\hline Alcohol & Isopropyl & 0.3 & 0.1 & 0.1 \\
\hline Aliphatic Hydrocarbon & Iso-Octane & 0.2 & 0.4 & 0.3 \\
\hline Amines & Pyridene & 0.2 & 0.5 & 0.5 \\
\hline Aromatic Hydrocarbon & Xylene (mixed) & 1.4 & 2.3 & 1.1 \\
\hline Chlorinated Aliphatic & Trichloroethylene & 0.5 & 0.8 & 0.8 \\
\hline Chlorinated Aromatic & Chlorobeneze & 1.1 & 1.5 & 1.5 \\
\hline Chlorinated Aromatic & O-Dichlorobeneze & 0.2 & 3.0 & 1.8 \\
\hline "Freon" & Trichlorotrifluoroethane & 0.2 & 0.2 & 0.2 \\
\hline Ketone & Acetone & 0.3 & 0.9 & 0.4 \\
\hline Keton & 2,4-Pentanedione & 0.6 & 1.2 & 1.4 \\
\hline
\end{tabular}

* Film strips were immersed in the test liquids for 90 minutes at room temperature and the thickness was re-measured by the IR method. In every case, equilibrium (no further thickness change) was reached before 90 minutes.

than that of silicone materials. Table II lists chemical compatibility results of Parylene films by film thickness measurements before and after immersion in different organic solvents for $90 \mathrm{~min}$ at room temperature [4]. Common solvents, especially those with similar chemical structures as Parylene, such as xylene, result in the most severe swelling of film thickness, which is still less than 3\% of the original parylene film thickness. Parylene $C$ shows excellent chemical resistance to aliphatic hydrocarbons such as octane, the primary component of engine fuel, only resulting in a swelling of $0.4 \%$.

\section{B. Interfacial Adhesion Promoters}

In addition to material considerations, interfacial adhesion reliability between polyimide passiviated Si-based substrate and silicone elastomer, silicone elastomer and Parylene $\mathrm{C}$ also play an important role in real-life applications. Also, the adhesion at various interfaces of the encapsulated MCM module must resist vibrations and air-stream impact during actual flight test.
Generally speaking, adhesion theory includes factors such as wet-out phenomena, weak boundary layers, polar adsorption, surface energies, and other effects. The predominant contributor to adhesion, however, is chemical bonding such as that introduced by organo-functional silane "coupling agents." These materials are used in very low concentration, but produce disproportionately large improvements in adhesion performance. Processing and fabrication conditions, which ensure the opportunity for interaction at both ends of the silane molecule, are essential to provide bonding on both sides of the interface [5], [6]. In this study, a total of 9 different functional silane coupling agents from Dow Corning Co. and Witco Corporation were used as candidate adhesion promoters. An appropriate mechanism is presented later in this study.

Since this is a long-term research project, reliability characterization and evaluation of the conformal coatings is still under study. This paper describes a preliminary aspect of the initial working results and reports a new bi-layer conformal coating design for similar potential applications.

\section{EXPERIMENTAL}

\section{A. SIR Test Vehicle and Conformal Coating Material Selection}

All the conformal coating materials applied in this project were initially evaluated by surface insulation resistance (SIR) measurements using an Alpha Metals SIR $\Omega$ meter (model 300 ) test instrument with custom copper/FR4 BellCore $Y$ comb test vehicles. The $50.8 \times 50.8 \mathrm{~mm}$ SIR board surface areas were coated with candidate materials immediately after a special cleaning process [7]. After encapsulation, the coated test boards were fixed into a Delp. Corporation 16 pattern SIR rack. Initial measurements were made to verify that the needle type test pins penetrated the coatings and made contact with the copper comb pattern. Finally, the test fixture and samples were mounted in a blue $\mathrm{M}$ humid-flow combination temperature and humidity cabinet set at $85^{\circ} \mathrm{C} / 85 \% \mathrm{RH}$. All coatings would be continuously exposed to $85^{\circ} \mathrm{C} / 85 \% \mathrm{RH}$ for $1000 \mathrm{~h}$, with $100 \mathrm{~V} \mathrm{dc}$ biasing.

The silicone candidates selected for this study were commercially available and the selection of coating materials was initially based on thermo-mechanical properties (under the condition of surviving temperature cycling from -55 to $\left.125^{\circ} \mathrm{C}\right)$ and level of mobile ionic impurities ( $<20 \mathrm{ppm}$ each of $\mathrm{Cl}^{-}, \mathrm{Na}^{+}$, and $\mathrm{K}^{+}$). All the candidate coatings experienced SIR measurements for further screening.

The two silicone elastomers selected for SIR testing include:

1) Dow Coming Q4-2817 fluorosilicone gel;

2) Dow Corning Hipec 3-6550 silicone gel.

The moisture and mobile ion resistance of Parylene $\mathrm{C}$ with a $5 \mu \mathrm{m}$ coating thickness was also tested by SIR in this study.

\section{B. Reliability Testing}

\section{I) Sample Preparation:}

a) Silicone elastomer coating: The polyimide passiviated Si-based substrates were cleaned using the same method as for SIR board treatment. Within $0.5 \mathrm{~h}$ after cleaning, the silicone materials were uniformly coated on the top of the 
cleaned substrates using a spin coater (Specialty Coating Systems, Inc. Model 6708D). Curing procedure for the Dow Corning Q4-2817 was $100^{\circ} \mathrm{C}$ for $30 \mathrm{~min}$. While, for Dow Corning Hipec $3-6550$ the curing procedure was $150^{\circ} \mathrm{C}$ for $60 \mathrm{~min}$.

b) Silane coupling agent Application: Direct pretreatment is the easiest and most satisfactory method to apply a silane to an inorganic surface. Two methods find extensive use.

1) Aqueous pretreatment, which is primarily used for fiberglass. Coating is accomplished by applying a dilute, aqueous solution of the silane, generally adjusted to $\mathrm{pH}$ 4 with acetic acid. The silane hydrolyzes and forms a layer of silanol molecules at the substrate surface. The solution, typically with a concentration of silane within $0.2-0.5 \%$, will ordinarily dissipate onto the surface. This method is generally performed with high efficiency.

2) Nonaqueous blending, which is widely used for mineral fillers. The silane, which is undiluted or mixed with a spreading solvent, is added to the inorganic substrate and thoroughly dry blended at low shear to give a uniform coating. Dry blending offers a convenient and effective route to coupling agent treatment of particulate inorganics such as alumina, silica, and glass microbeads used as fillers in composites. A silane treatment level of between $0.5-1.0 \%$ is appropriate for most common minerals. The additive method is an alternate route commonly used to apply silane coupling agents. With this method, the silane is added to the polymeric phase from which it diffuses or migrates to the inorganic surface. Reaction occurs first by hydrolysis, and then by condensation to produce siloxane coupling linkages. Uniform treatment is dependent upon thorough blending of the mixture and filler. Typically, the silane is added at a concentration of $1.0 \%$ or less.

In regard to our unique bi-layer RWOH design, the three kinds of silane application methods discussed above were not appropriate. To address this problem, a new silane application method, named as vapor deposition, was used to directly deposit silane on the surface of the silicone elastomer. This method was selected to avoid film swelling and still produce a uniform adhesion promoter layer. Consequently, Parylene $\mathrm{C}$ is promptly polymerized on top of the silane while reactivity still sustains.

Prior to silane application, all the silicone coated substrates were treated with UV-ozone at $50^{\circ} \mathrm{C}$ for $5 \mathrm{~min}$. Immediately following treatment, the side with the silicone elastomer was exposed to the silane solutions. Silane solutions were then heated between $60-70{ }^{\circ} \mathrm{C}$ for $20 \mathrm{~min}$ to vaporize sufficient silane which was uniformly condensed on the entire surface of the silicone elastomers. An excess of silane will lead to a swelling in the silicone coating. After that, the samples were immediately placed into a Parylene deposition system, Lab Coater 2010, from Special Coating System Co. for Parylene $\mathrm{C}$ deposition.

c) Parylene $C$ deposition: Parylene $\mathrm{C}$ deposition was conducted using the Gorham Method. The Parylene $C$ coating process starts with the vaporization of the dimer at $150^{\circ} \mathrm{C}$,

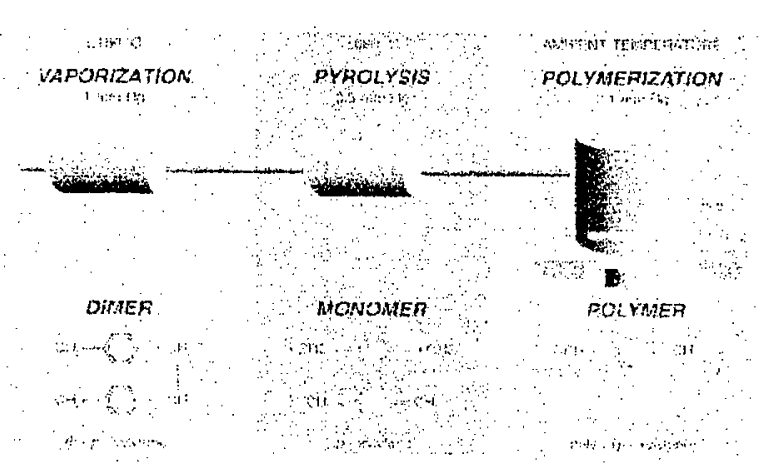

Fig. 1. Schematic graph of Parylene C deposition processes.

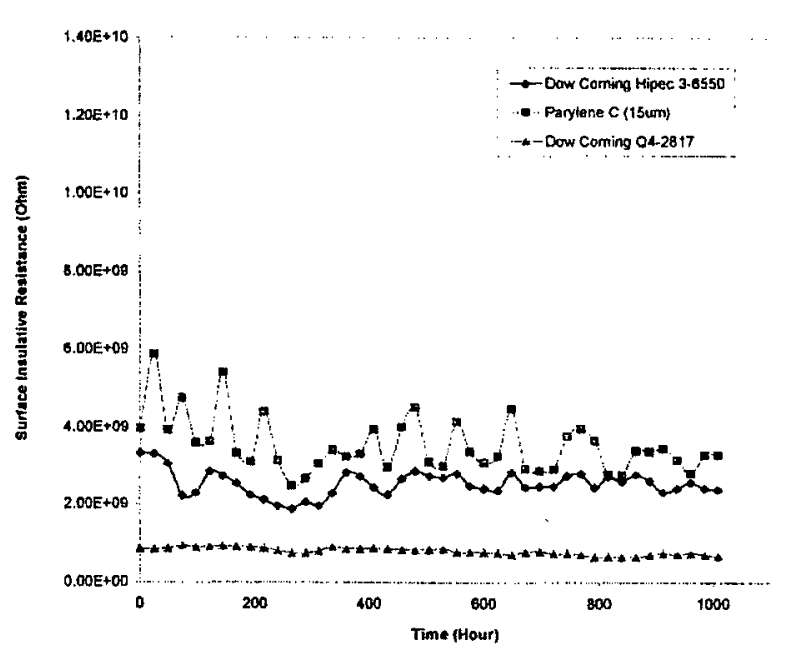

Fig. 2. Surface insulative resistance of Parylene $\mathrm{C}$ and silicone.

0.1 Torr. The resulting dimer gas is further elevated to $650^{\circ} \mathrm{C}$ into the pyrolysis zone to obtain two methylene bonds which generate a stable para-xylylene. Finally, the monomer is sent to the ambient temperature deposition vacuum chamber where the para-xylene polymerizes and coats on the surface of the silicone elastomers [8]. A Schematic graph of the Parylene C deposition process is illustrated in Fig. 1.

2) Reliability Testing: All the single layer or bilayer shear strength test samples were exposed to $85^{\circ} \mathrm{C} / 85 \% \mathrm{RH}$ (relative humidity) chamber (Blue M, model no. AC-7602HA) for 1000 $h$ and thermal shock chamber (thermotron environmental test chamber, ATS-320-DD-10-705-LN2) for 500 cycles. Thermal cycling was set from -55 to $125{ }^{\circ} \mathrm{C}$. The time for each temperature ramp was $15 \mathrm{~min}$, and the dwell period at either -55 or $125^{\circ} \mathrm{C}$ was $15 \mathrm{~min}$. The adhesion test interval for $85^{\circ} \mathrm{C} / 85 \% \mathrm{RH}$ aging is $200 \mathrm{~h}$, and for thermal stressing is 100 cycles.

3) Adhesion Strength Test: For the adhesion test, small dies with size of $2 \times 2$ mil were attached on top of the bilayer conformal coating using an epoxy resin as an adhesive, which was dried at room temperature. Since the interfacial adhesion between die and Parylene $C$ is strong enough that the delamination could only occur between the Parylene $C$ and silicone elastomers. Shear strength test was performed with an adhesion analyzer (Royce Instruments System 552) at room 
TABLE III

Candidate Silane Coupling Agents for Interfacial adhesion Enhancement

\begin{tabular}{|c|c|c|}
\hline Designation & Chemical Name & Chemical Formula \\
\hline A. 174 & $\begin{array}{l}\text { y-Methacry loxypropyl } \\
\text { trimethoxy silane }\end{array}$ & $\mathrm{CH}_{2}=\mathrm{C}\left(\mathrm{CH}_{3}\right)_{\mathrm{CO}} \mathrm{CH}_{2} \mathrm{CH}_{2} \mathrm{CH}_{2} \mathrm{Si}\left(\mathrm{OCH}_{3}\right)_{3}$ \\
\hline$A-1120$ & $\begin{array}{l}N-\beta-(\text { Aminoethy })-y \text {-amino } \\
\text { propyltrimethoxysilane }\end{array}$ & $\mathrm{H}_{2} \mathrm{NCH}_{2} \mathrm{CH}_{2} \mathrm{NHCH}_{2} \mathrm{CH}_{2} \mathrm{CH}_{2} \mathrm{Si}_{2}(\mathrm{OCH})_{3}$ \\
\hline PS $\times 5 \$ 4$ & & $\left(\mathrm{H}_{2} \mathrm{NCH}_{2}\right)_{2} \mathrm{Si}\left(\mathrm{OCH}_{2} \mathrm{CH}_{3}\right)_{2}$ \\
\hline$Y .9669$ & $\begin{array}{l}\mathrm{N} \text {-Phenyl-y-am in opropy! } \\
\text { trimelhoxy silane }\end{array}$ & $\left.-1-\mathrm{NH} \mathrm{CH} \mathrm{CH}_{2} \mathrm{CH}_{2} \mathrm{Si}_{2} \mathrm{OCH}_{3}\right)_{3}$ \\
\hline A -1102 & $\gamma$-Aminopropyltricthylsilane & $\mathrm{H}_{2} \mathrm{NCH}_{2} \mathrm{CH}_{2} \mathrm{CH}_{2} \mathrm{~S}_{2}\left(\mathrm{OCH}_{2} \mathrm{CH}_{3}\right)_{3}$ \\
\hline$A-171$ & Vinyltrine thoxysilane & $\mathrm{CH}_{2}=\mathrm{CHSi}(\mathrm{OCH})_{3}$ \\
\hline$A-191$ & Vinyltriethoxysilane & $\mathrm{CH}_{2}=\mathrm{CHS}\left(\mathrm{OCH}_{2} \mathrm{CH}_{3}\right)_{3}$ \\
\hline$A-172$ & Vinyl-tris-(2-Inetho $x$ yethoxy)sil & $\mathrm{CH}_{2}=\mathrm{CHS}\left(\mathrm{OCH}_{2} \mathrm{CH}_{2} \mathrm{OCH}_{3}\right)_{3}$ \\
\hline$A-2120$ & $\begin{array}{l}N-\beta \cdot(\text { aminoethy } 1)-\% \text { aninopropyl } \\
\text { methyldimethoxysilane }\end{array}$ & $\left.\mathrm{H}_{2} \mathrm{NCH}_{2} \mathrm{CH}_{2} \mathrm{CH}_{2} \mathrm{CH}_{2} \mathrm{Si}\left(\mathrm{CH}_{3}\right)_{2} \mathrm{OSi}_{2} \mathrm{CH}_{3}\right)_{2} \mathrm{CH}_{2} \mathrm{CH}_{2} \mathrm{CH}_{2} \mathrm{CH}_{2} \mathrm{NH}_{2}$ \\
\hline
\end{tabular}

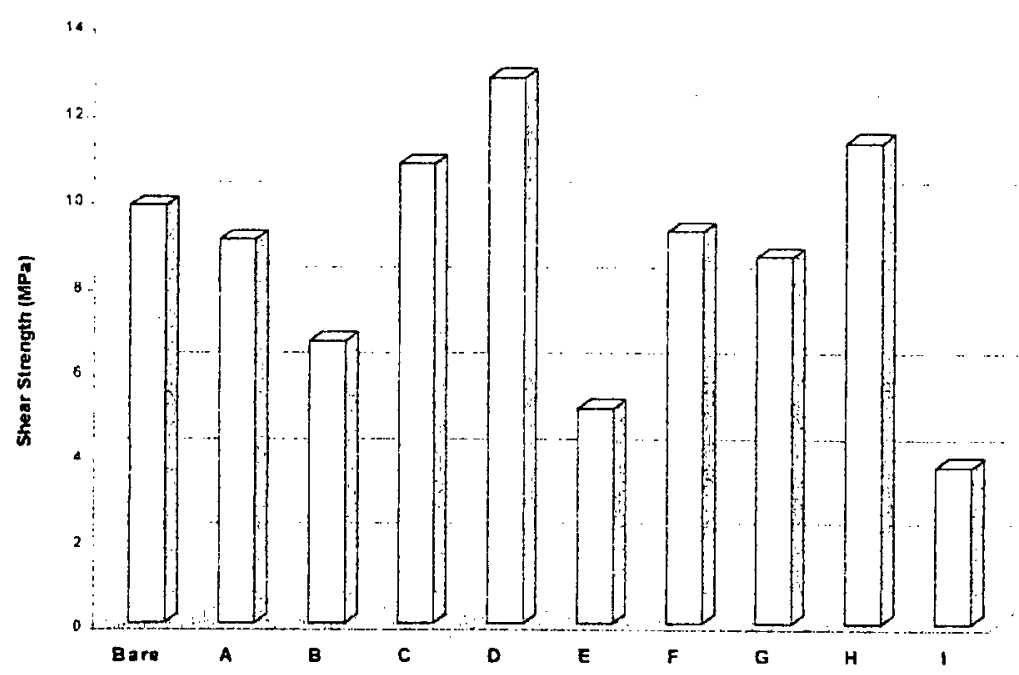

Fig. 3. Shear strength between Parylene $C$ and Dow Corning Q4-2817 elastomer with various silane coupling agents after sample preparation.

temperature. Testing was conducted at a speed of $0.1 \mathrm{~mm} / \mathrm{s}$ $(0.004 \mathrm{in} / \mathrm{s})$ with a vertical offset of $49 \mu \mathrm{m}(0.002 \mathrm{in})$.

\section{RESULTS AND DISCUSSION}

SIR measurement results in Fig. 2 indicate that the Dow Corning Hipec 3-6550, Q4-2817 and Parylene C conformal coatings pass the aerospace specification limit of $1 E 08 \Omega$. The surface insulation resistance shows a slowly decreasing trend during the testing period. This can be attributed to moisture and mobile ion ingress facilitating mobile ion migration from cathode to anode during biasing, which will result in resistance decrease.

To improve the adhesion strength between the silicone elastomer and Parylene $\mathrm{C}$, nine different functional silane coupling agents were selected as adhesion promoter candidates between Parylene $\mathrm{C}$ and Silicone Elastomer based on chemical covalent relation between silane organic functional groups and Parylene $\mathrm{C}$ chemical structure. Chemical structures of all nine silane coupling agents are listed in Table III. 


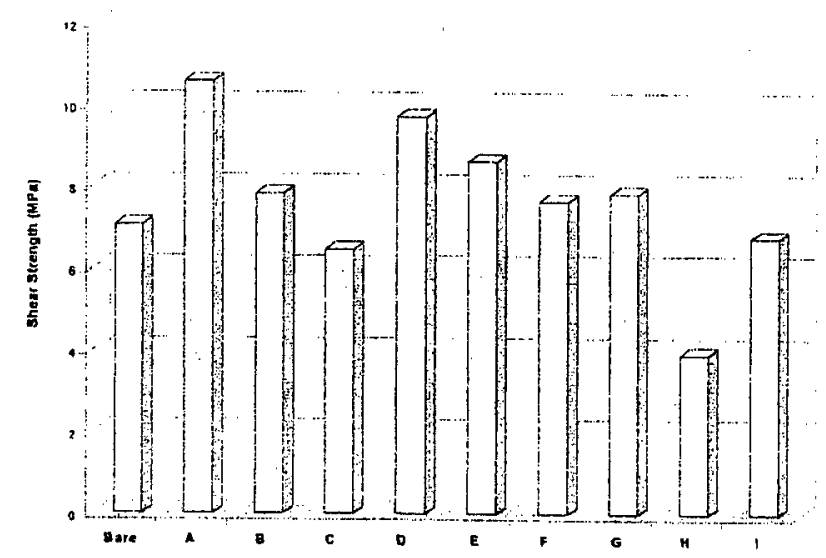

Fig. 4. Shear strength between Parylene C and Dow Corning Hipec 3-6550 elastomer with various silane coupling agents after sample preparation.

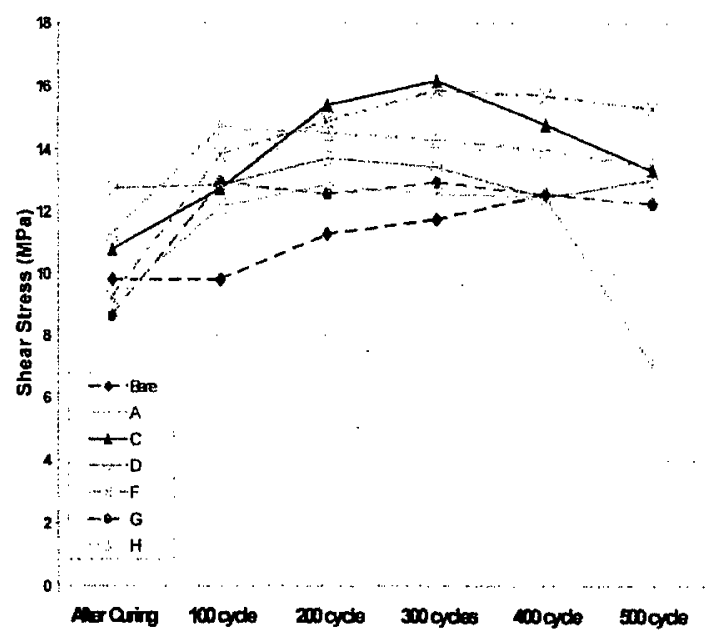

Fig. 5. Shear strength between Parylene $C$ and Dow Coming Q4-2817 elastomer with various silane coupling agents after exposure to thermal shock aging.

Shear strengths between Parylene C and Dow Corning Q42817 after sample preparation using various silane coupling agents as adhesion promoters are illustrated in Fig. 3. Results indicate that shear strengths obviously increase using silane coupling agents $\mathrm{C}, \mathrm{D}$, and $\mathrm{H}$, and shear strengths decrease using silane coupling agents $\mathrm{A}, \mathrm{F}$, and $\mathrm{G}$. Shear strengths greatly decrease using silane coupling agent $\mathrm{B}, \mathrm{E}$, and $\mathrm{I}$. Therefore, samples with silane coupling agents $A, C, D, F$, $\mathrm{G}$, and $\mathrm{H}$ require further reliability testing. Shear strengths between Parylene C and Dow Corning Hipec 3-2817 after sample preparation are shown in Fig. 4. Results indicate that shear strengths increase using silane coupling agents $A, B, D$, $\mathrm{E}, \mathrm{F}$, and $\mathrm{G}$. Shear strengths decrease using silane coupling agents $\mathrm{C}$ and $\mathrm{I}$, and Shear strengths greatly decrease using silane coupling agent $\mathrm{H}$. Subsequently, samples with silane coupling agents A, B, C, D, E, F, G, and I will be subjected to further reliability testing.

Shear strength results of samples submitted to thermal cycling are shown in Fig. 5 after 100 cycles. Shear strength

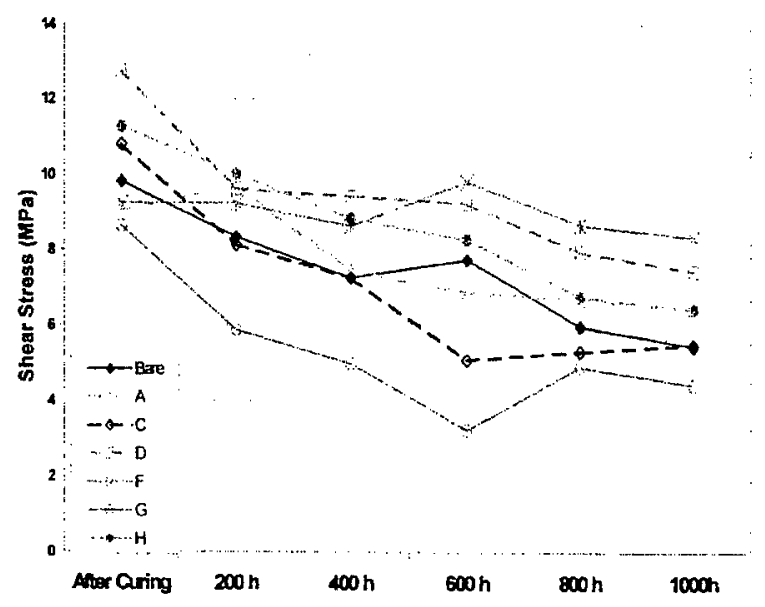

Fig. 6. Shear strength between Parylene $C$ and Dow Corning Q4-2817 elastomer with various silane coupling agents after exposure to $85^{\circ} \mathrm{C} / 85 \%$ RH stressing.

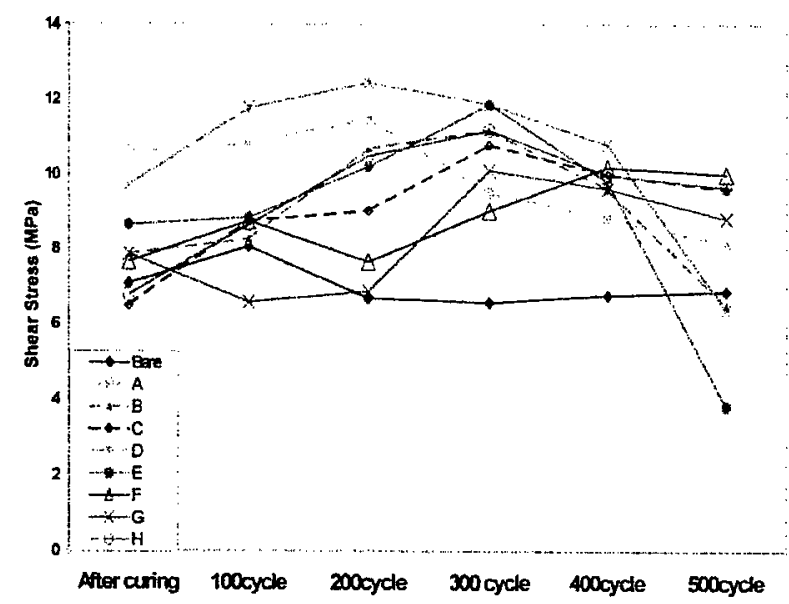

Fig. 7. Shear strength between Parylene $C$ and Dow Corning Hipec 3-6550 elastomer with various silane coupling agents after exposure to thermal shock aging.

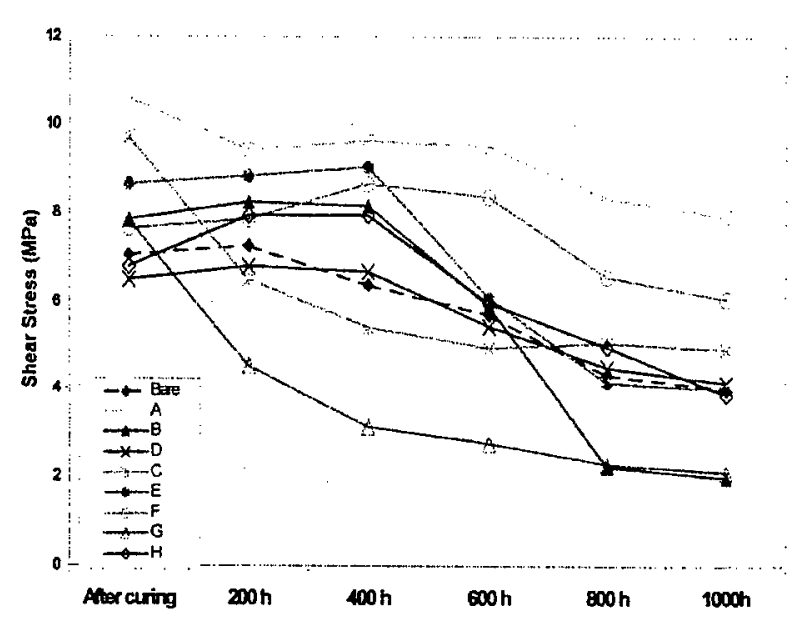

Fig. 8. Shear strength between Parylene $C$ and Dow Coming Hipec 3-6550 elastomer with various silane coupling agents after exposure to $85^{\circ} \mathrm{C} / 85 \%$ RH stressing. 

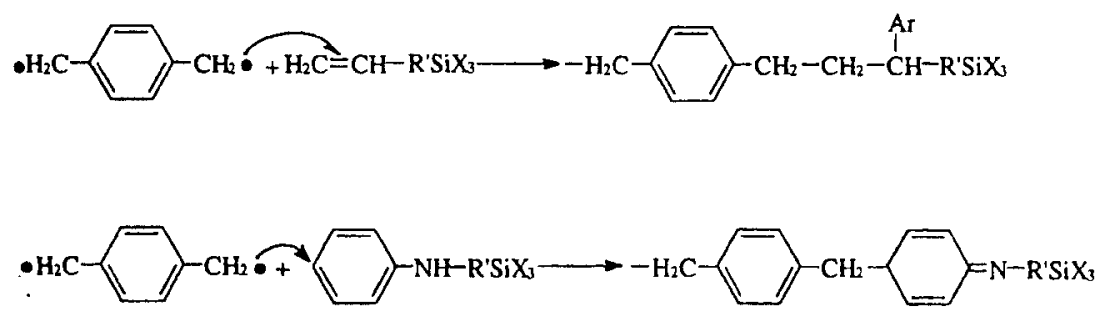<smiles>CCc1ccc(CC#[W])cc1</smiles>

$\left.\mathrm{R}-\mathrm{SiOCH}_{3}\right)_{3}+3 \mathrm{H}_{2} \mathrm{O} \rightleftharpoons \mathrm{R}-\mathrm{Si}(\mathrm{OH})_{3}+3 \mathrm{HO}_{-} \mathrm{CH}_{3}$

$\left(\mathrm{H}_{3} \mathrm{CCOO}\right)_{3}-\mathrm{Si}_{-} \mathrm{CH}_{3}+3 \mathrm{H}_{2} \mathrm{O}=\mathrm{H} 3 \mathrm{C}-\mathrm{Si}(\mathrm{OH})_{3}+3 \mathrm{H}_{3} \mathrm{CCOOH}$

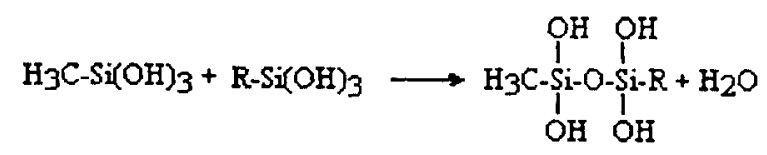

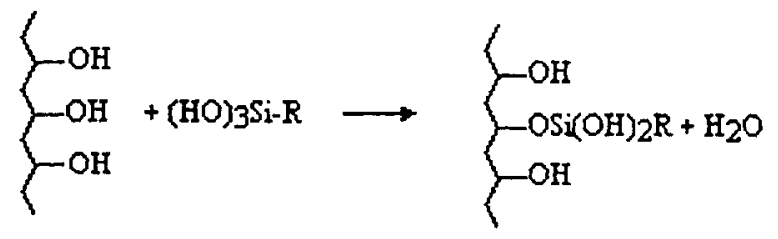

Fig. 9. Equations (I)-(V).

results between Parylene $\mathrm{C}$ and Dow Coring Q4-2817 after $85^{\circ} \mathrm{C} / 85 \% \mathrm{RH}$ aging are shown in Fig. 6 at $200 \mathrm{~h}$ intervals. From Fig. 5, it is observed that the shear strength of most samples increases after exposure to thermal cycling for 100 cycles. The possible reason for this is that the intrinsic cross-linking reaction of the silicone elastomer is maintained under thermal cycling during this period, and some cyclic oligomers, such as D3, D4, and D5, etc. are continuously diffused out. As a result, the modulus increase of the silicone elastomer leads to a shear strength increase. However, after sample exposure to thermal cycling for a considerable time, CTE mismatch between Parylene C (35-40 ppm) and silicone elastomer (300-500 ppm) intensified and causes microdelamination to occur at some interfacial sites, which finally become the failure centers during shear testing and the interfacial adhesion is lowered. While shear strengths for most samples continuously decrease under $85^{\circ} \mathrm{C} / 85 \% \mathrm{RH}$ aging due to the modulus of silicone elastomer reduction as shown in Fig. 6. This situation may be the result of two factors. One possible reason is that when the bilayer sample is kept under $85^{\circ} \mathrm{C}$ for an extended time, some low molecular silicone cyclics may be continuously generated and trapped in the cross-linked network as plasticizers, since the high moisture pressure intuitively prohibits the cyclics outward diffusion. Another possible reason is that the moisture may cause the siloxane to inversely hydrolyze into silanols at $85^{\circ} \mathrm{C}$. The moisture came from permeation of moisture in $85 \%$ $\mathrm{RH}$ atmosphere through the Parylene $\mathrm{C}$ sheared (removed) areas during prior die shear testing.

From analysis of the results from Figs. 5 and 6, it can be concluded that the best adhesion promoters for the Parylene $\mathrm{C}$ and Dow Corning Q4-2817 combination are silane coupling agents $\mathrm{D}$ and $\mathrm{F}$.

Shear strength results of the Parylene $\mathrm{C}$ and Hipec 36550 bi-layer combination after different intervals of thermal cycling and $85^{\circ} \mathrm{C} / 85 \% \mathrm{RH}$ aging are shown in Figs. 7 and 8 , respectively. The shear strength trends are the same as those between Parylene C and Dow Corning Q4-2817. It can be observed that the best two silane coupting agents for Parylene $\mathrm{C}$ and Dow Coming Hipec 3-6550 are silane coupling agent $\mathrm{A}$ and $\mathrm{F}$ as confirmed by adhesion reliability testing. 


\section{Mechanism for SLlane Coupling AGENTS AS ADHESION PROMOTERS}

Silane coupling agents are a family of organosilicon monomers, which are characterized by the formula: $\mathrm{R}-\mathrm{Si} X_{3}$. In this formula, $\mathrm{R}$ is an organofunctional group attached to silicon in a hydrolytically stable manner, and $X$ designates hydrolyzable groups that are converted to silanol groups by hydrolysis.

Among these nine silanes, the ones that contain a vinyl, methacryloxypropyl, or phenyl amino-functional group at one end, and a methoxy-functional group at another end will function as the best three adhesion promoters.

These silanes chemically bond to the methylene linkage of Parylene $\mathrm{C}$ through radical-initiated polymerization. The possible bonding mechanisms are shown in (I) and (II) in Fig. 9. The di-para-xylylene radical that comes from Parylene $\mathrm{C}$ thermal pyrolysis maintains high reactivity before polymerization. This free radical will readily attack the $c=c$ double bond according to Markownikoff's free radical mechanism [9].

For the anilino group, since the amino function group is an electron-donating group and its lone electron-pair on the nitrogen atom in the $\mathrm{P}$ orbit will conjugate with an electron " $\pi$ cloud" above or below the benzene rings. The electron-density on the phenyl ring will be higher than that of the normal condition, which will make the benzene ring more active. As a result, the xylylene free radical group will selectively bond to the carbon atom according to the steric site under the allow of Markownikoff Law.

On the other end, since methoxy is the smallest and active group bonded to the $\mathrm{Si}$ atom, it is easily hydrolyzed into silanol [see (III) in Fig. 9]. Since there are components such as silica filler and methyltriacetoxysilane in Dow Corning Q42817 and Hipec 3-6550, the silanol group would condense with the methyltriacetoxysilane hydrolyzed silanol group or directly bond to the surface of the silica [see (4) and (5), respectively].

\section{CONCLUSION}

A novel bilayer conformal coating for MEMS device encapsulation has been designed in accordance with the requirement of RWOH. Parylene $\mathrm{C}$ is a promising conformal coating for aerospace application. Adhesion between Parylene $\mathrm{C}$ and silicone elastomer has been intensified by applying silane coupling agents at the interface as adhesion promoters. The adhesion promoted coupling reliability has also been investigated by die shear testing after thermal shock stressing and $85^{\circ} \mathrm{C} / 85 \% \mathrm{RH}$ aging. Test results indicate that the silane species with a vinyl or anilino group at one end and a methoxy group at the other end will show the best adhesion enhancement. A proper mechanism was also presented.

\section{REFERENCES}

[1] C. P. Wong, Polymers for Electronic and Photonic Applications. San Diego, CA: Academic, 1993.

[2] "Parylene Coated Elastomers," U.S. Patent 5075174, Dec. 24, 1991.
[3] R. Olson, "Parylene conformal coating and its applications for electronics," in Proceedings of the Symposium on the Interface. Fairfax Station, VA: Interface Foundation of North America, 1991, p. 112.

[4] Parylene technical data website: http://www.paratronix.com/tdata/.

[5] E. P. Plueddemann, "Adhesion through silane coupling agents," in Proc. 25th Annu. SPI PR/C Conf., 1970, sect. 13-D, pp. 1-10.

[6] P. E. Cassidy and W. J. Yager, "A review of coupling agents as adhesion promoters," NASA Contract NAS8-24073, TRACOR Document T-70AU-7129-U, pp. 13-16, 1992.

[7] C. P. Wong and R. McBride, "Pre-encapsulation cleaning methods and control for microelectronics packaging," IEEE Trans. Comp., Manufact., Packag. Technol. A, vol. 17, p. 542, Dec. 1994.

[8] L. You, G. R. Yang, C. I. Lang, P. Wu, and J. A. Moore, "Vapor deposition of Parylene films from precursors," in Proc. 3rd Biennial Meeting Chem. Perspectives Microelectron. Mater., Boston, MA, 1992.

[9] Theoretical Organic Chemistry, C. Parkanyi, Ed. New York: Elsevier, 1998.

Jiali Wu received the B.S. degree in chemistry from Zhejiang University, China, and the M.S. and Ph.D. degrees in chemistry from the Shanghai Institute of Metallurgy, Chinese Academy of Science, Beijing.

From 1991 to 1997, her major research work focused on microsensor fabrication and application in electrochemistry field and die bonding with $\mathrm{Au} / \mathrm{In}$ bi-alloy isothermal solidification technique. She joined the Electronics Packaging Research Group, School of Materials Science and Engineering, Packaging Research Center, Georgia Institute of Technology, Atlanta, as a Postdoctoral Fellow, in 1997. She is currently working on multilayer conformal coatings for reliability without hermeticity, reworkable high temperature adhesives for MCM-D processing, parylene application, and its adhesion enhancement on polymeric substrates using silane as coupling agents.

Randy T. Pike (M'99) received the B.A. degree from the State University of New York, Oswego, the M.S. degree in chemistry from the Rochester Institute of Technology, Rochester, NY, in 1996, and is currently pursuing the $\mathrm{Ph} . \mathrm{D}$. degree in materials science and engineering at the Georgia Institute of Technology, Atlanta.

From 1993 to 1994, he worked in the field of organic photoreceptor analysis and development at the Xerox Corporation. From 1994 to 1996, he studied synthetic strategies to novel liquid crystalline polyimides for aerospace applications. He has co-authored several additional papers on adhesives for MCM-D application. His current thrusts are in reliable non-hermetic conformal coatings, and he is currently working with the Boeing Company on MEMS device encapsulation.

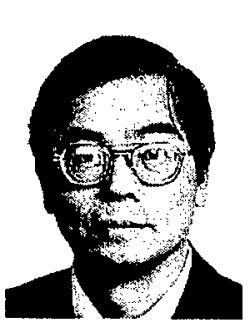

C. P. Wong (SM'87-F'92) received the B.S degree in chemistry from Purdue University, West Lafayette, IN, and the Ph.D. degree in organic/inorganic chemistry from Pennsylvania State University, University Park.

After his doctoral study, he was awarded two years as a Postdoctoral Scholar at Stanford University, Stanford, CA. He joined AT\&T Bell Laboratories, Murray Hill, NJ, in 1977, as Member of Technical Staff. He has been involved with the research and development of polymeric materials (inorganic and organic) for electronic applications. He became Senior Member of Technical Staff in 1982, Distinguished Member of Technical Staff in 1987, and an AT\&T Bell Laboratories Fellow in 1992. His research interest lie in the fields of polymeric materials, high Tc ceramics, material reaction mechanism, IC encapsulation in particular, hermetic equivalent plastic packaging and electronic manufacturing packaging, and reliability processes. He holds over 30 U.S. patents and numerous international patents, and has published over 80 technical papers and 90 presentations in the related area.

Dr. Wong was the IEEE Components, Packaging, and Manufacturing Technology (CMPT) Society Technical President in 1997 and 1998. He currently serves as Chair of the IEEE Technical Activities Board Steering Committee on Design and Manufacturing Engineering. 


\title{
Interface-Adhesion-Enhanced Bi-layer Conformal Coating for Avionics Application \\ - Parylene C Coated Silicone Elastomer
}

\author{
Jiali Wu, Randy T. Pike, C.P. Wong \\ School of Materials Science and Engineering \\ \& \\ Packaging Research Center \\ Georgia Institute of Technology \\ Atlanta, GA 30332-0245 \\ Phone: 404-894-8391 \\ Fax: 404-894-9140
}

\begin{abstract}
A flexible, smooth, and low profile conformal coating was developed to fulfill the encapsulation of a Microelectromechanical System (MEMS) which will be applied to sense the static pressure on airplane during real flight test. The encapsulation should be able to protect the MEMS device and the MCM module from adverse environment, i.e. mechanical shock, temperature fluctuation, engine fuel and oil contamination, and moisture/mobile ion permeation. Conventional packaging for electronics could not satisfy this particular out-door application, and a new encapsulant combination has been designed in accord with the requirement of Reliability Without Hermeticity (RWOH). A bi-layer structure was selected because of the property limitation of single material. Silicones, as typically pliable elastomer material, are featured of flexible, water repelling, and abrasion resistance. It will be first applied to flatten the MEMS surface and function as durable dielectric insulation, stress-relief, and shock/vibration absorbers over a large humidity/temperature range. To compensate the deficiency of silicone on engine fuel/oil contamination, parylene $\mathrm{C}$ is to be deposited afterward. This bi-layer coating can achieve excellent bulk properties, such as moisture and mobile ion barrier, chemical compatibility, and electrical insulation characteristics. However, the poor adhesion of parylene $C$ to silicone greatly restricts its application. To address this problem, silane coupling agents were used as adhesion promoter. Significant adhesion improvement was achieved by placing an interlayer of silane coupling agent to provide interfacial bonding to the silicone elastomeric surface and the parylene $\mathrm{C}$ film. Furthermore, a possible mechanism of adhesion enhancement will also be presented in this study.
\end{abstract}

Key words: Microelectromechanical System (MEMS), MCM module, bi-layer conformal coating, silicone elastomer, parylene $\mathrm{C}$, silane coupling agent. 


\section{INTRODUCTION}

Long-term, reliable protection of sensitive circuits and components is becoming more important in many of today's delicate and demanding electronic application. The need of low profile, flexible, high accuracy and economical packaging for Microelectromechanical System (MEMS)-based modules protection results in a new conformal coating design for Reliability Without Hermeticity (RWOH). General organic passivation materials such as polyimides, silicones, and epoxy have been used as RWOH encapsulants with reasonable success. However, these organics are generally permeable to moisture. Moisture diffusion rates through organic polymeric encapsulants can be in minutes, hours, or days. The rate depends on the type of material, the material thickness, and the length of diffusion time. Moisture on the surface of the devices, in the presence of mobile ions such as $\mathrm{Na}^{+}, \mathrm{K}^{+}$, and $\mathrm{Cl}^{-}$will result in device failure. [1] To ensure the $\mathrm{RWOH}$, we propose to combine a low moisture permeable silicone elastomer coating with a thin Para-X-xylyene (Parylene C) to ensure long-term protection. The silicone elastomer is coated as a relatively thick layer (approximately 10mil) primarily for the delicate circuit protection and as a planarizing layer of the MEMS.

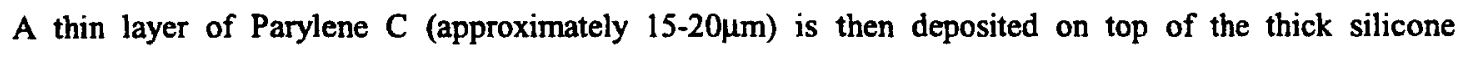
elastomer layer. This combination provides an outstanding barrier to moisture and mobile ion $\left(\mathrm{Na}^{+}, \mathrm{K}^{+}, \mathrm{Cl}\right)$ permeation and an aerodynamically smooth surface for avionics application. Furthermore, low surface energy of parylene as a top coating can effectively avoid air borne particles and fibers absorption induced contamination. [2]

\section{Conformal Coating Materials}

Silicones, as typically pliable elastomer materials, can function as durable dielectric insulation, as barriers against environmental contamination, and as stress relieving shock/vibration absorbers over a very humidity/temperature range. In this study, Dow Coming Q1-2817 and DC3-6550 were selected as candidate elastomers, which especially function as top profile planar agent.

Parylene as a new member of conformal coating family has a high dielectric strength and excellent chemical resistance. [3] Currently, parylene contains three kinds of commercial products, parylene N, C and D. Unlike conventional liquid coating, parylene coatings are applied by vapor deposition in vacuum chamber at ambient temperature. This unique method of processing of vapor deposition can form $100 \%$ 
pinhole-free uniform coverage and has the ability to penetrate and coat very complex geometry. Coating thickness is also easily controlled. In this study, Parylene $C$ is selected as the candidate in terms of moisture permeation resistance, chemical resistance and cost consideration. [4]

Table llists the normalized permeability of gasses through polymer materials. The permeability of Parylene $\mathrm{C}$ is especially much lower than almost any other engineering plastics. Moisture permeability resistance of Parylene $\mathrm{C}$ is as 40 times as that of silicone materials. Table 2 lists the chemical compatibility results of parylene films by film thickness measurement before and after immersion in different kinds of organic solvents for $90 \mathrm{~min}$ at room temperature. The common solvents, especially those with similar chemical structure as parylene such as xylene, cause a most severe swelling of film thickness, which is less than $3 \%$ of original parylene film thickness. While the major component of engine fuel/oil, aliphatic hydrocarbon such as octane, only cause a swelling of $0.4 \%$.

\section{Interfacial Adhesion Promoters}

Besides material consideration, interfacial adhesion reliabilities between polyimide passiviated Si-based substrate and silicone elastomer, silicone elastomer and Parylene $C$ also play an important role in real application. Adhesion at various interfaces of the encapsulated MCM module device must keep well for resist vibration and air-stream impact during real flight test.

Generally speaking, adhesion theory includes many factors such as wet-out phenomena, weak boundary layers, polar adsorption, surface energies, and other effects. The predominant contributor to adhesion, however, is chemical bonding such as that introduced by organiofunctional silane "coupling agents". These materials are used in very low concentration, but produce disproportionately large improvements in adhesion performance. Processing and fabrication conditions, which ensure the opportunity for interaction at both ends of the silane molecule, are essential to provide bonds on both sides of the interface. [5] Silane brocure In this study, totally 9 kinds of different functional silane coupling agents from Dow Corning Co. and $\mathrm{Witco} C \mathrm{C}$. were used as candidate adhesion promoters. And an appropriate mechanism will be presented thereafter.

Since this is a long-teim research project, reliability characterization and evaluation of the conformal coating is still under progressing. This paper just describes an aspect of the initial working results and reports a new bi-layer conformal coating design for the potential similar applications. 


\section{EXPERIMENTS}

\section{A. SIR Test Vehicle and Conformal Coating Material Selection}

All the conformal coating materials applied for this project were initially evaluated by Surface Insulation Resistance (SIR) with time using Alpha Metals SIR $\Omega$ meter (Model 300) test vehicle. The BellCore SIR test board has a special $\mathrm{Pt} / \mathrm{Ph}$ interdigital comb pattern deposited on it. The whole surface area of SIR board was coated with candidate materials immediately after special cleaning process. [6] Then the coated board was clamped on a SIR rack, and made sure that the needle type pins penetrated the coatings and tightly attached to the $\mathrm{Pt} / \mathrm{Rh}$ wire of the comb pattern. Finally, the whole rack together with the samples was put into the $85^{\circ} \mathrm{C} / 85 \%$ chamber later. All the coatings would be continuously exposed to $85^{\circ} \mathrm{C} / 85 \%$ relative humidity condition for $1000 \mathrm{~h}$, and all the data would be collected once an hour under 100 volt biasing.

Silicone the candidates selected for this test were available on the commercial market, the selection of coating material was initially based on thermo-mechanical properties (surviving temperature cycling from $55^{\circ} \mathrm{C}$ to $\left.125^{\circ} \mathrm{C}\right)$ and level of mobile ionic impurities $\left(<20 \mathrm{ppm}\right.$ each of $\mathrm{Cl}^{-}, \mathrm{Na}^{+}$, and $\left.\mathrm{K}^{+}\right)$. All the candidate coatings would experienced SIR measurement for further screening.

Two silicone elastomers were selected for further relibilitics test: 1) Dow Corning DC4-2817 silicon gel, 2) Dow Corning DC3-6550 silicone gel. The moistuse and mobile ion resistance of Panglene $C$ with 35 un thiekness was also testod by SIR in this study.

\section{B. Reliability Test}

\section{1) Sample preparation}

\section{a. Silicone elastomer coating}

The polyimide passivated Si-based substrates were cleaned using the same method as that for SIR board treatment. Then the silicone materials were uniformly coated on the top of the cleaned substrates by a spin coater (Specialty Coating Systems, Inc. Model 6708D). Curing procedure for Dow Corning Q1-2817 was $100^{\circ} \mathrm{C}$ for $30 \mathrm{~min}$. While, for Dow Coming DC3-6550 was $150^{\circ} \mathrm{C}$ for $60 \mathrm{~min}$.

\section{b. Silane Coupling Agent Applying}

Direct pretreatment is the earliest and most satisfactory method to apply a silane to an inorganic surface. [7] Two methods find extensive use: 1) Aqueous pretreatment, which specially used for fiberglass. A dilute, 
aqueous solution of the silane, generally adjusted to $\mathrm{pH} 4$ with acetic acid, is contacted with the glass substrate with which it rapidly reacts, forming a layer of silanol molecules at the surface. The solution, typically with a concentration of silane within 0.2 to $0.5 \%$, will ordinarily exhaust onto the surface, reflecting the excellent efficiency of the method. 2) Nonaqueous blending, which widely used for mineral fillers. The silane, which undiluted or mixed with a spreading solvent, is added to the inorganic substrate and thoroughly dry blended at low shear to give a uniform coating. Dry blending offers a convenient and effective route to coupling agent treatment of particulate inorganics such as alumina, silica and glass microbeads used as fillers in composites. A silane treatment level of between 0.5 and $1.0 \%$ is appropriate for most common minerals. The additive method is the other route commonly used to apply silane coupling agents. The silane is added to the polymeric phase from which it diffuses or migrates to the inorganic surface or reacts, first by hydrolysis, and then by condensation to siloxane coupling linkages. Uniform treatment depends upon thorough blending of the mixture, including filler. Typically the silane is added at a concentration of $1.0 \%$ or less.

As to our unique bi-layer RWOH design, all the above three kinds of silane applying method are not appropriate. To address this problem, a new silane applying method -vapor deposition is used to directly deposit silane on the surface of silicone elastomer for avoiding film swelling and a uniform adhesion promoter layer is formed. Consequently, Parylene $\mathrm{C}$ is promptly polymerized on top of silane before its reactivity still sustaines.

Prior to silane applying, all the silicone coated substrates were treated with UV-ozone at $50^{\circ} \mathrm{C}$ for $5 \mathrm{~min}$. After that, the side with silicone elastomer was immediately faced down to the silane aquents solutions. Then silane aqueous solutions were heated to $60^{\circ} \mathrm{C}$ to $70^{\circ} \mathrm{C}$ for $20 \mathrm{~min}$ to have enough amount of silane vaporized and uniformly condensed on the whole surface of silicone elastomers. Any over-quantity of silane will lead to swelling in silicone coating. Then put these samples into PDS, Lab Coater 2010 chamber (Parylene Deposition System, Lab Coater 2010, from Special Coating System Co.) for Parylene C deposition, immediately.

\section{c. Parylene C Deposition}

Parylene $C$ deposition was conducted by PDS, Lab Coater 2010. The Parylene C coating process starts with the vaporization of the dimer at $150^{\circ} \mathrm{C}, 0.1 \mathrm{Tor}$. The resulting dimer gas is further elevated at $650^{\circ} \mathrm{C}$ in the 
pyrolysis zone to obtain two methylene bonds to generate a stable para-xylylene. Finally the monomer is sent to the ambient temperature deposition vacuum chamber where the para-xylene polymerizes and coats on the surfaces of the silicone elastomers. [8] Schematic graph of parylene C deposition process is illustrated in Figure 1.

\section{2) Reliability Test}

All the single layer or bilayer shear stress test samples will be exposed to $85^{\circ} \mathrm{C} / 85 \% \mathrm{RH}$ (Relative Humidity) chamber for $1000 \mathrm{~h}$ and Thermal Shock chamber for 500 cycle. Thermal shock cycling was set from $-55^{\circ} \mathrm{C}$ to $125^{\circ} \mathrm{C}$, time for temperature ramp is $15 \mathrm{~min}$, and dwelling period at either $-55^{\circ} \mathrm{C}$ or $125^{\circ} \mathrm{C}$ was $15 \mathrm{~min}$. Adhesion test interval for $85^{\circ} \mathrm{C} / 85 \% \mathrm{RH}$ aging is $200 \mathrm{~h}$, for thermal shock stressing is 100 cycle.

\section{RESULTS AND DISCUSSION}

SIR measurement results in Figure 2 need to be drawn indicates that all the Dow Corning DC3-6550, Q42817 and Parylene $\mathrm{C}$ conformal coatings pass the specification limit of $1 \mathrm{E} 08 \mathrm{Ohms}$ for surface insulative resistance $\left(\Delta R / \mathrm{R}_{0} \geq 1 \times 10^{8} \Omega\right)$ after exposure to $85^{\circ} \mathrm{C} / 85 \% \mathrm{RH}$ for $1000 \mathrm{~h}$ according to the MIL...standard. And the surface insulation resistance continuous decreasing within testing period. Because the moisture and mobile ion ingression facilitates the mobile ion migration from cathode to anode during biasing, which will result in resistance decrease. The bare SIR board was used as control sample.

To improve the adhesion strength between silicone elastomer and Parylene $\mathrm{C}$, nine kinds of different functional silane coupling agents were selected as adhesion promoter candidates based on chemical covalent relation between silane organic founctional group and Parylene $\mathrm{C}$ chemical structure. Chemical structures of all the nine kinds of silane coupling agent were listed in Table 3.

Shear stresses between Parylene $C$ and Dow Coring Q4-2817 using various silane coupling agents as adhesion promoters are illustrated in Figure 3. after sample preparation. Results indicate that shear stresses increase obviously using silane coupling agent PSX554, Y-9669 and A-172, shear stresses decrease a little bit using silane coupling agent $A-174, A-171$ and $A 151$, and shear stresses greatly decrease using silane coupling agent A-1120, A-1102 and A-2120. So, samples with silane coupling agent PSX554, Y-9669, A172, A-174, A-171 and A151 would further go through reliability test. While shear stresses between Parylene C and Dow Coring DC3-2817 after sample preparation are shown in Figure 4. Results indicate that shear stresses increase using silane coupling agent A-174, A-1120, Y-9669, A-1102 A-171 and A-151, 
shear stresses a little bit decrease using silane coupling agent PSX554 and A-2120, and shear stresses greatly decreased using silane coupling agent A-172. So, samples with silane coupling agent A-174, A1120, Y-9669, A-1102, A-171, A-151 and A-2120 would further go through reliability test.

Shear stresses results of samples submitted to thermal shock chamber are shown in Figure 5 with a 100 cycle interval. And shear stresses results between Parylene $\mathrm{C}$ and Dow Coring Q4-2817 after $85^{\circ} \mathrm{C} / 85 \%$ $\mathrm{RH}$ aging are shown in Figure 6 with a 200h interval. From Figure 6 we can observe that the shear stresses of most samples increase after exposure to thermal shock cycling for 100 cycles. The possible reason for this is that the intrinsic cross-linking reaction of silicone elastomer keeps going under thermal shock condition during this period, and some oligomers, such as D3, D4 and D5, etc. are continuously formed and diffuse outward to the various interfaces at the same time as well. As a result the modulus increase of silicone elastomer leads to the shear stress increasing. However, after the samples exposure to thermal cycling for too long time, CTE mismatching between Parylene $\mathrm{C}$ and silicone elastomer causes microdelamination appeared at some interfacial sites, which finally become the failure centers during shear test and lower the interfacial adhesion. While shear stresses of most samples continuously decrease under $85^{\circ} \mathrm{C} / 85 \% \mathrm{RH}$ aging condition due to the modulus of silicone elastomer reduction. This situation may be caused by two reasons. One possible reason is that when the bilayer sample is kept under $85^{\circ} \mathrm{C}$ for over long time, some cyclics may be continuously generated and trapped in the cross-link network as plastizers, since the high moisture pressure probably prohibit the cyclics outward diffusion. Another possible reason is that the moisture maybe cause the siloxane inversely hydrolyzed into silanols at $85^{\circ} \mathrm{C}$. The moisture came from permeation of moist in $85 \% \mathrm{RH}$ atmosphere through the parylene $\mathrm{C}$ sheared (removed) areas during prior die shear test.

Compromise the results of Figure 5 and Figure 6, It can be concluded that the best adhesion promoters for Parylene C and Dow Coming Q4-2817 combination are Y-9669 and A-171.

Shear stress results of Parylene C and DC3-6550 bilayer combination after different intervals of thermal shock aging and $85^{\circ} \mathrm{C} / 85 \%$ RH stressing are shown in Figure 7 and Figure 8, respectively. Variation trends of shear stress are the same with that between Praline $C$ and Dow Corning Q4-2817. It can be observed that the best two silane coupling agents for Praline C and Dow Coming DC3-6550 are A-174 and A-171 confirmed by adhesion reliability tests. 


\section{MECHANISM FOR SILANE COUPLING AGENTS AS ADHESION PROMOTERS}

Silane coupling agents are a family of organosilicon monomers, which are characterized by the formula: $R$ $\mathrm{SiX}_{3}$. In this formula, $\mathrm{R}$ is an organofunctional group attached to silicon in a hydrolytically stable manner, and $\mathrm{X}$ designates hydrolyzable groups, which are converted to silanol groups on hydrolysis.

Among these nine kinds of silanes, the ones which containing vinyl, methacryloxypropyl or phenyl aminofunctional group at one end, and methoxy-functional group at another end will function as the best three adhesion promoters. They chemically bond to methylene of Parylene $\mathrm{C}$ through radical-initiated polymerization. Their possible bonding mechanisms are shown in equation (II) and (III). Di-p-xylylene radical that comes from Parylene $\mathrm{C}$ thermal pyrolysis remains high reactivity before polymerization (I). This free radical will readily attack vinyl groups according to Markownikoff's free radical mechanism.

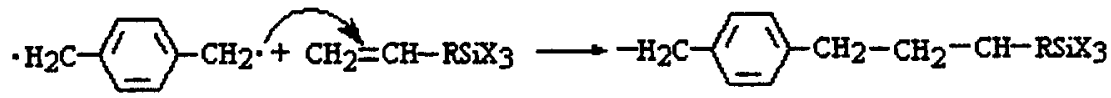

(I)

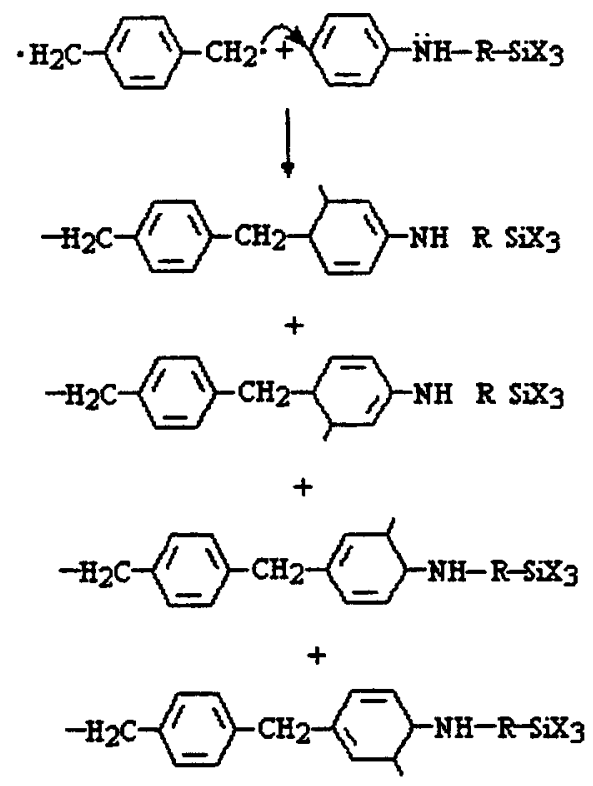

(II) 
For the anilino group, since the amino is electron-repel group and the remote electron-pair on nitrogen atom in P orbit will conjugate with electron "cloud" above or below the benzene rings. The electron-density on phenyl ring will be higher than that of normal condition, which will make the benzene ring more active, so the xylylene free radical group will selectively bond to the carbon atom according to the stereo space. On the other end, since methoxy is the smallest group bonded to $\mathrm{Si}$ atom, it is easily hydrolyzed into silanol (Equation (III)). Since there are components such as silica filler and methyltriacetoxysilane in Dow Corning Q4-2817 and DC3-6550, the silanol group would condense with methyltriacetoxysilane hydrolyzed silanol group or directly bond to the surface of the silica. (Equation (IV), (V)).

$\mathrm{R}-\mathrm{Si}(\mathrm{OCH})_{3}+3 \mathrm{H}_{2} \mathrm{O} \rightleftharpoons \mathrm{R}-\mathrm{Si}(\mathrm{OH})_{3}+3 \mathrm{HO}-\mathrm{CH}_{3}$

$\left(\mathrm{H}_{3} \mathrm{CCOO}\right)_{3}-\mathrm{Si}-\mathrm{CH}_{3}+3 \mathrm{H}_{2} \mathrm{O} \rightleftharpoons \mathrm{H} C \mathrm{C}-\mathrm{Si}(\mathrm{OH})_{3}+3 \mathrm{H}_{3} \mathrm{CCOOH}$

$\mathrm{H}_{3} \mathrm{C}-\mathrm{Si}(\mathrm{OH})_{3}+\mathrm{R}$-Si(OH) $)_{3} \longrightarrow \mathrm{H}_{3} \mathrm{C}-\mathrm{Si}-\mathrm{O}-\mathrm{Si}_{1} \mathrm{R}$

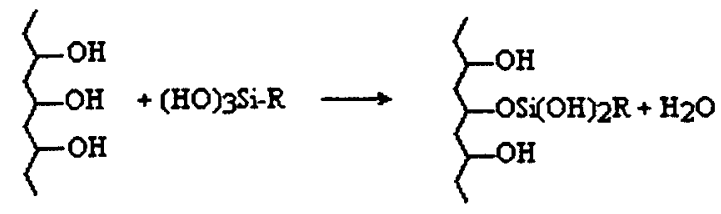

(III)

(IV)

(v)

\section{CONCLUSION:}

A novel bilayer conformal coating for MEMS device encapsulation has been designed in accord with the requirement of RWOH. Parylene as a promising conformal coating find a new applicable field in this study. Adhesion between parylene $\mathrm{C}$ and silicone elastom $\mathrm{r}$ has been intensified by applying silane coupling agents at the interface as adhesion promoters, and its reliability has also been investigated by die shear test after interval thermal shock and $85^{\circ} \mathrm{C} / 85 \% \mathrm{RH}$ aging. Test result indicates that the silane species with vinyl 
or anilino group at one end and methoxy group at the other end will play the best adhesion enhancement. And proper mechanism for this has been put forth.

\section{ACKNOWLEDGEMENT}

The support from Boeing Company is greatly appreciated.

\section{REFERENCE}

[1] C.P.Wong, Ed. "Polymers for Electronic and Photonic Applications", Academic Press, San Diego, CA 1993

[2] US Patent, PN: 5075174, "Parylene Coated Elastomers".

[3] Roger Olson, "Parylene Conformal Coating and It's Applications for Electronics", Proceedings of the Symposium on the Interface, Published by Interface Foundation of North America Fairfax Station VA USA p 112,(1991)

[4] Parylene technical data web site: http://www.paratronix.com/tdata/

[6] C.P. Wong and R. McBride, "Preencapsulation Cleaning Methods and Control for Microelectronics Packaging", IEEE Transaction on CMPT, Part A, Vol. 17-4, p. 542 (1994)

[7] silane applying

[8] L. You, G. R. Yang, C.I. Lang, P. Wu, and J.A. Moore, "Vapor deposition of parylene films from precursors", Proceedings of the 3rd Biennial Meeting of Chemical Perspectives of Microelectronic Materials 11/30-12/03/92, Boston, MA, USA 
Table 1 Normalised permeability of gasses through polymer materials

Table 2 Swelling caused by organic solvents at room temperature

Table 3 Candidate Silane Coupling Agents for Enhancing Adhesion between Parylene C and Silicone Elastomer

Figure 1. Schematic graph of Parylene $\mathrm{C}$ deposition process

Figure 2 Surface insulative resistances of parylene $\mathrm{C}$ and silicone Elastomers varied with time under 100 volts biasing.

Figure 3 Shear stress between Parylene $C$ and Dow Corning Q4-2817 elastomer with various silane coupling agents after sample preparation

Figure 4 Shear stress between Parylene C and Dow Corning DC3-6550 elastomer with various silane coupling agents after sample preparation

Figure 5 Shear stress between Parylene C and Dow Coming Q4-2817 elastomer with various silane coupling agents after exposure to thermal shock aging

Figure 6 Shear stress between Parylene C and Dow Corning Q4-2817 elastomer with various silane coupling agents after exposure to $85^{\circ} \mathrm{C} / 85 \% \mathrm{RH}$ stressing

Figure 7 Shear stress between Parylene $C$ and Dow Coming DC3-6550 elastomer with various silane coupling agents after exposure to thermal shock aging

Figure 8 Shear stress between Parylene $C$ and Dow Corning DC3-6550 elastomer with various silane 
coupling agents after exposure to $85^{\circ} \mathrm{C} / 85 \% \mathrm{RH}$ stressing

Table 1 Normalised permeability of gasses through polymer materials $\mathrm{H}_{2} \mathrm{O}$ in $\mathrm{cm}^{3} \cdot \mathrm{mm} / \mathrm{m}^{2}$.day.atm. All gasses in g.mm/ $\mathrm{m}^{2}$.day.atm

\begin{tabular}{|c|c|c|c|c|c|}
\hline & $\mathrm{O}_{2}$ & $\mathrm{~N}_{2}$ & $\mathrm{CO}_{2}$ & $\mathrm{H}_{2}$ & $\mathrm{H}_{2} \mathrm{O}$ \\
\hline Parylene C & 2.8 & 0.4 & 3 & 43 & 0.08 \\
\hline Parylene D & 12 & 1.8 & 5 & 94 & 0.1 \\
\hline Parylene N & 15 & 3 & 84 & 213 & 0.6 \\
\hline HDPE & 73 & 17 & 228 & n.d. & 0.12 \\
\hline PS & 138 & 23 & 400 & n.d. & 3.5 \\
\hline PTFE & 223 & 133 & n.d. & 516 & n.d. \\
\hline LDPE & 140 & 80 & 700 & n.d. & 0.6 \\
\hline PC & 124 & 22 & 827 & n.d. & 1.5 \\
\hline
\end{tabular}




\begin{tabular}{|c|c|c|c|c|c|}
\hline FEP & 295 & 126 & 657 & 381 & 0.16 \\
\hline Silicone & 19000 & n.d. & 118000 & 17000 & 3 \\
\hline
\end{tabular}

Table 2 Swelling caused by organic solvents at room temperature

\begin{tabular}{|l|l|c|c|c|}
\hline \multicolumn{2}{|c|}{ Solvents } & \multicolumn{3}{c|}{ \% Swelling Parylene } \\
\hline \multicolumn{1}{|c|}{ Class } & \multicolumn{1}{|c|}{ Test Member } & $\mathrm{N}$ & $\mathrm{C}$ & $\mathrm{D}$ \\
\hline Alcohol & Isopropyl & 0.3 & 0.1 & 0.1 \\
\hline Aliphatic Hydrocarbon & Iso-Octane & 0.2 & 0.4 & 0.3 \\
\hline Amines & Pyridene & 0.2 & 0.5 & 0.5 \\
\hline Aromatic Hydrocarbon & Xylene (mixed) & 1.4 & 2.3 & 1.1 \\
\hline Chlorinated Aliphatic & Trichloroethylene & 0.5 & 0.8 & 0.8 \\
\hline Chlorinated Aromatic & Chlorobeneze & 1.1 & 1.5 & 1.5 \\
\hline Chlorinated Aromatic & O-Dichlorobeneze & 0.2 & 3.0 & 1.8 \\
\hline "Freon" & Trichlorotrifluoroethane & 0.2 & 0.2 & 0.2 \\
\hline Ketone & Acetone & 0.3 & 0.9 & 0.4 \\
\hline Keton & 2,4-Pentanedione & 0.6 & 1.2 & 1.4 \\
\hline
\end{tabular}

* Film strips were immersed in the test liquids for 90 minutes at room temperature and the thickness was re-measured by the IR method. In every case, equilibrium (no further thickness change) was reached before 90 minutes. 
Table 3 Candidate Silane Coupling Agents for Enhancing Adhesion between Parylene $\mathrm{C}$ and Silicone Elastomer 


\begin{tabular}{|c|c|c|}
\hline Designation & Chemical Name & Chemical Formula \\
\hline A- 174 & $\begin{array}{l}\text { Y-M ethacrylox ypropyl } \\
\text { trimethoxysilane }\end{array}$ & 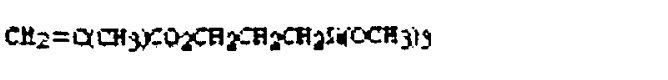 \\
\hline A-1120 & $\begin{array}{l}N-\beta \text {-(A minoethyl)- } \gamma \text {-amino } \\
\text { propyltrimethoxysilane }\end{array}$ & 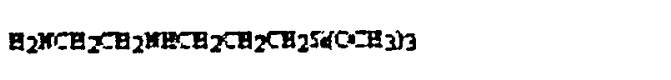 \\
\hline PSX554 & & $\mathrm{H}_{2} \mathrm{HCH}_{2} \mathrm{H}_{2} \mathrm{OCH}_{2} \mathrm{CH}_{3} \mathrm{H}_{2}$ \\
\hline$Y-9669$ & $\begin{array}{l}\text { N-Phenyl- } \gamma \text {-am inopropyl } \\
\text { trimethoxysilane }\end{array}$ & $\sqrt{13}-\mathrm{ABCB}_{2} \mathrm{CH}_{2} \mathrm{OB}_{25} \mathrm{OCOH}_{313}$ \\
\hline$A-1102$ & $\gamma$-A minopropyltriethylsilane & 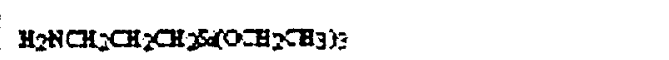 \\
\hline A-171 & Vinyltrimethoxysilane & $\mathrm{CH}_{\mathrm{i}}=\cot \mathrm{COCO}_{1} \mathrm{O}$ \\
\hline$A-151$ & Vinyltrie thoxysilane & $\left.\mathrm{CH}_{2}=\mathrm{CES}_{2} \mathrm{OCH}_{2} \mathrm{CH}_{3}\right)_{3}$ \\
\hline$A-172$ & Vin $y$ l-tris-(2-metho $x$ yethox $y)$ sil & $\mathrm{CH}_{2}=\mathrm{FAMOCH}_{2} \mathrm{CH}_{2} \mathrm{OH} 3 \mathrm{OS}$ \\
\hline A-2120 & $\begin{array}{l}N-\beta \text {-(aminoethyl)- } \gamma \text {-aminopropyl } \\
\text { methyldimethoxysilane }\end{array}$ & 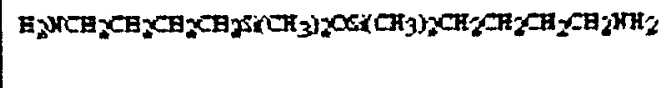 \\
\hline
\end{tabular}




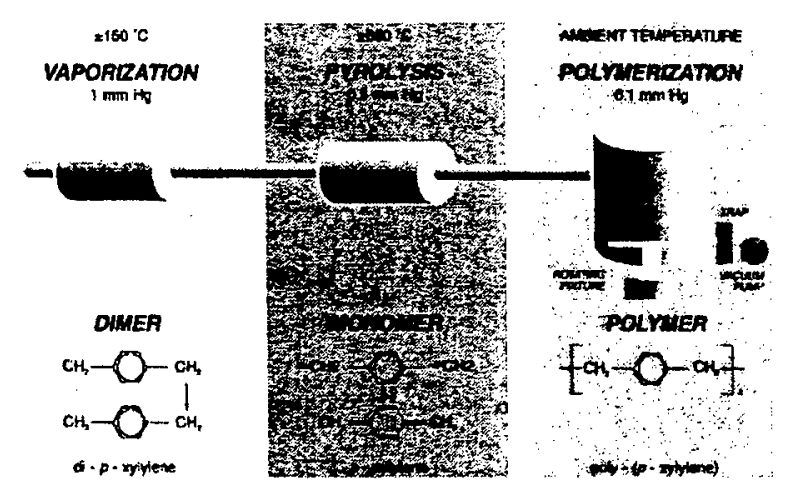

Figure 1. Schematic graph of Parylene $\mathrm{C}$ deposition process

Figure 2 Surface insulative resistances of parylene $C$ and silicone Elastomers varied with time under 100 volts biasing

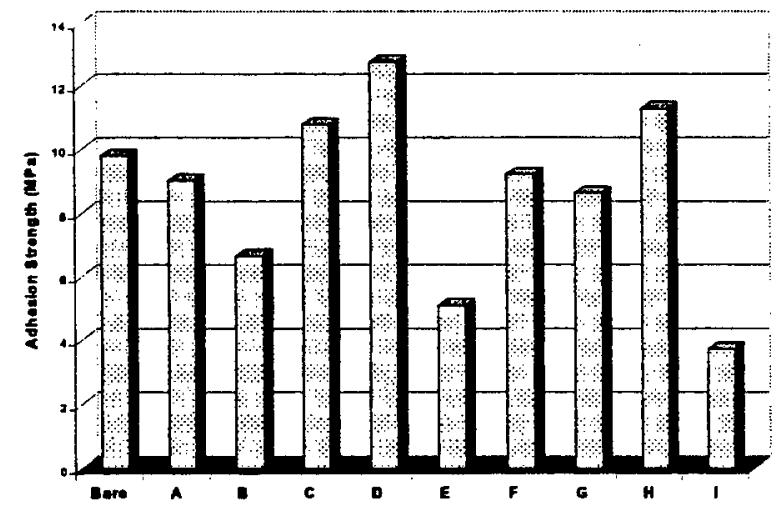

Figure 3 Shear stress between Parylene C and Dow Corning Q4-2817 elastomer with various silane coupling agents after sample preparation 


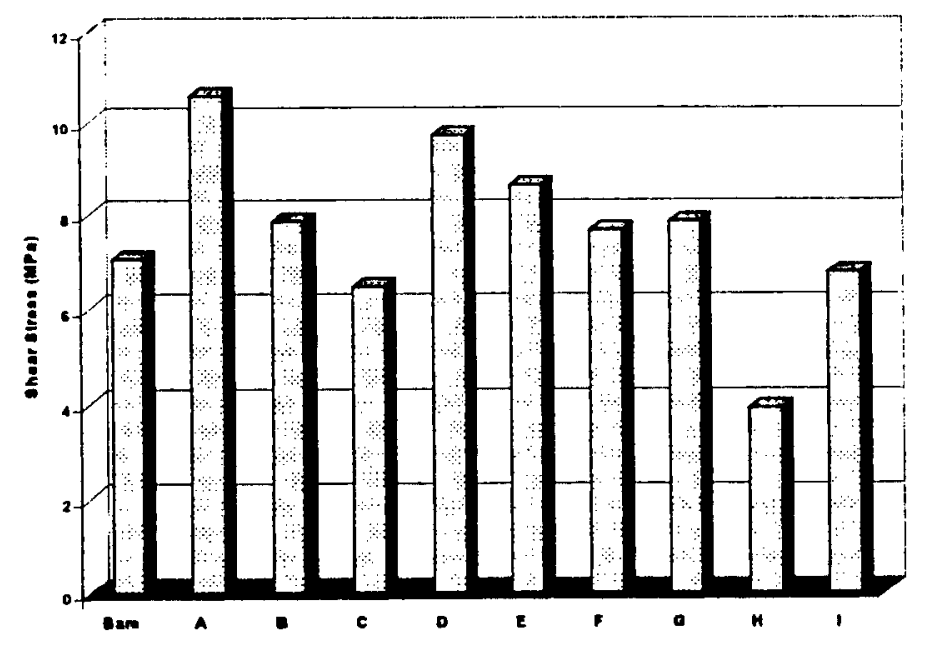


Figure 4 Shear stress between Parylene $C$ and Dow Corning DC3-6550 elastomer with various silane coupling agents after sample preparation 


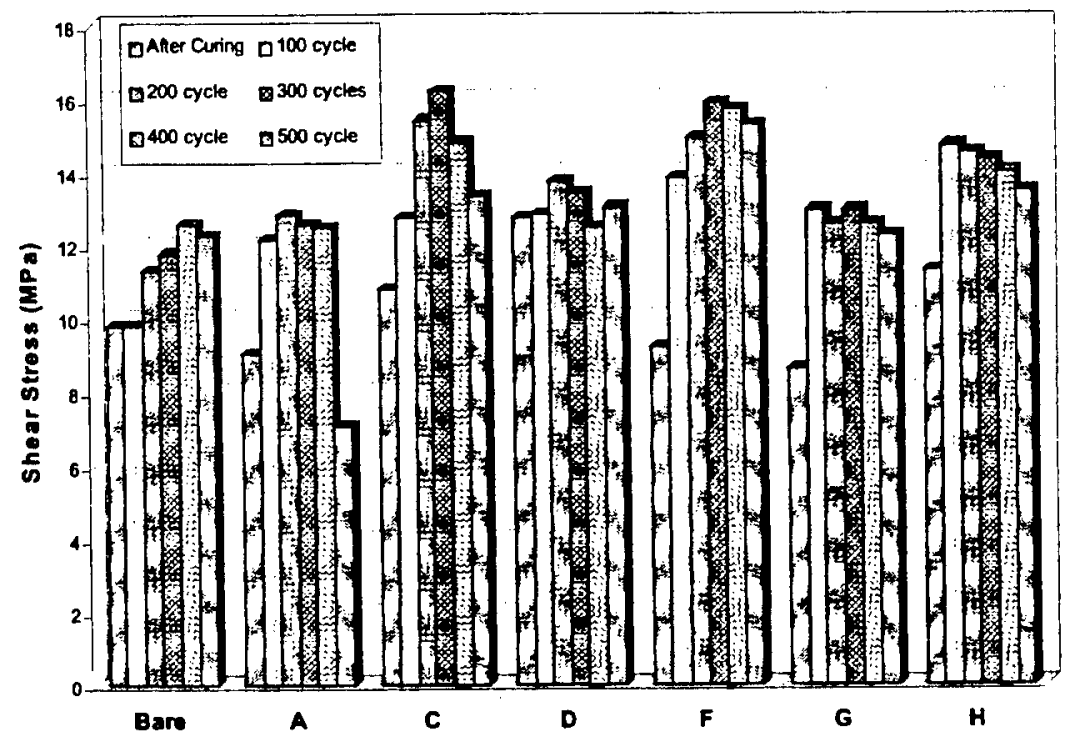

Figure 5 Shear stress between Parylene C and Dow Corning Q4-2817 elastomer with various silane coupling agents after exposure to thermal shock aging 


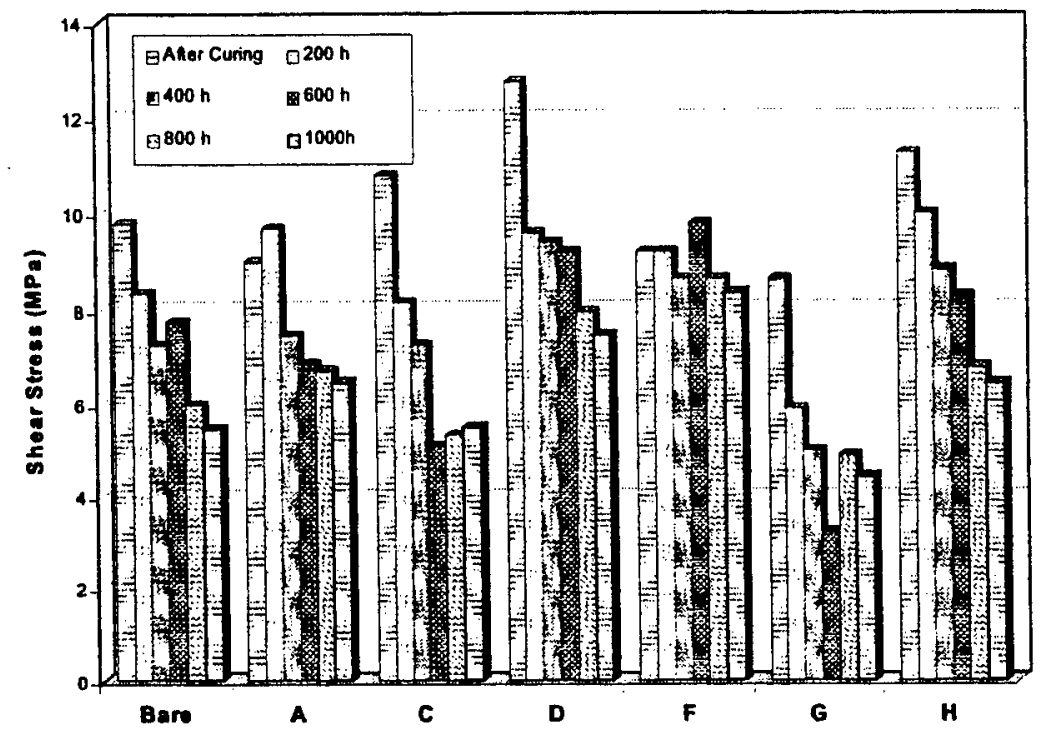

Figure 6 Shear stress between Parylene $C$ and Dow Corning Q4-2817 elastomer with various silane coupling agents after exposure to $85^{\circ} \mathrm{C} / 85 \% \mathrm{RH}$ stressing 


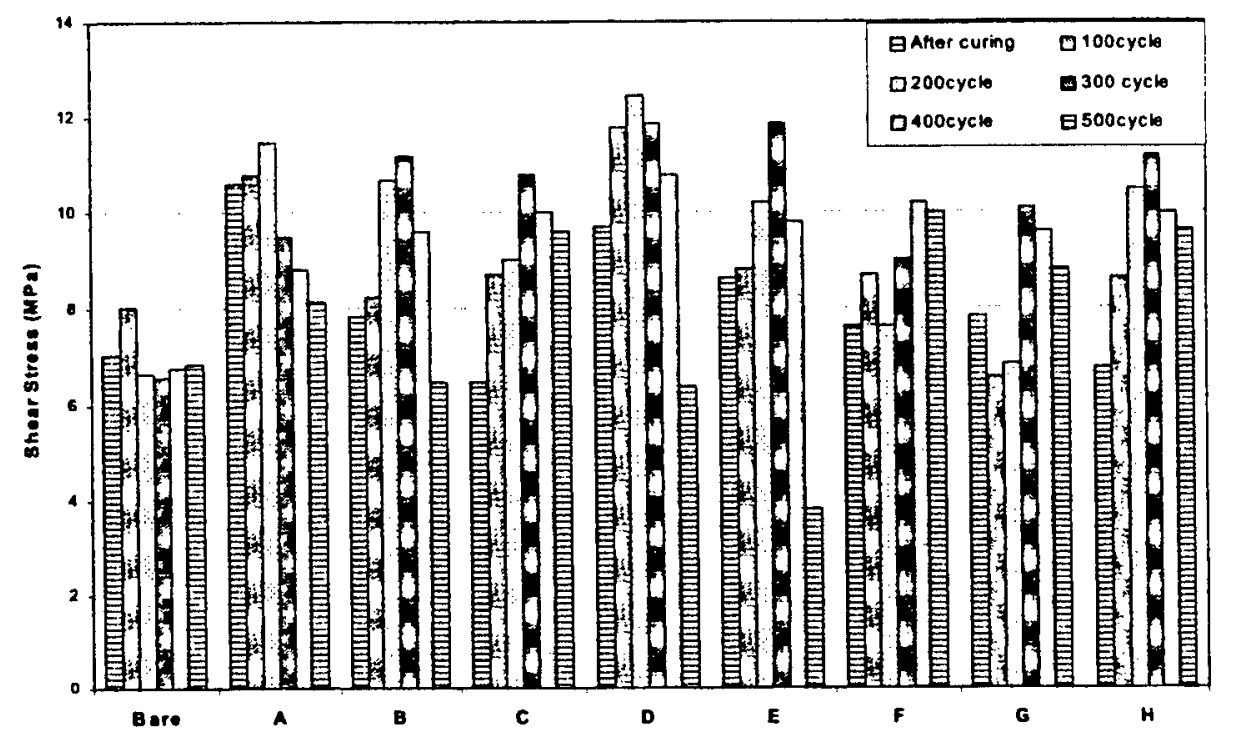

Figure 7 Shear stress between Parylene C and Dow Corning DC3-6550 elastomer with various silane coupling agents after exposure to thermal shock aging 


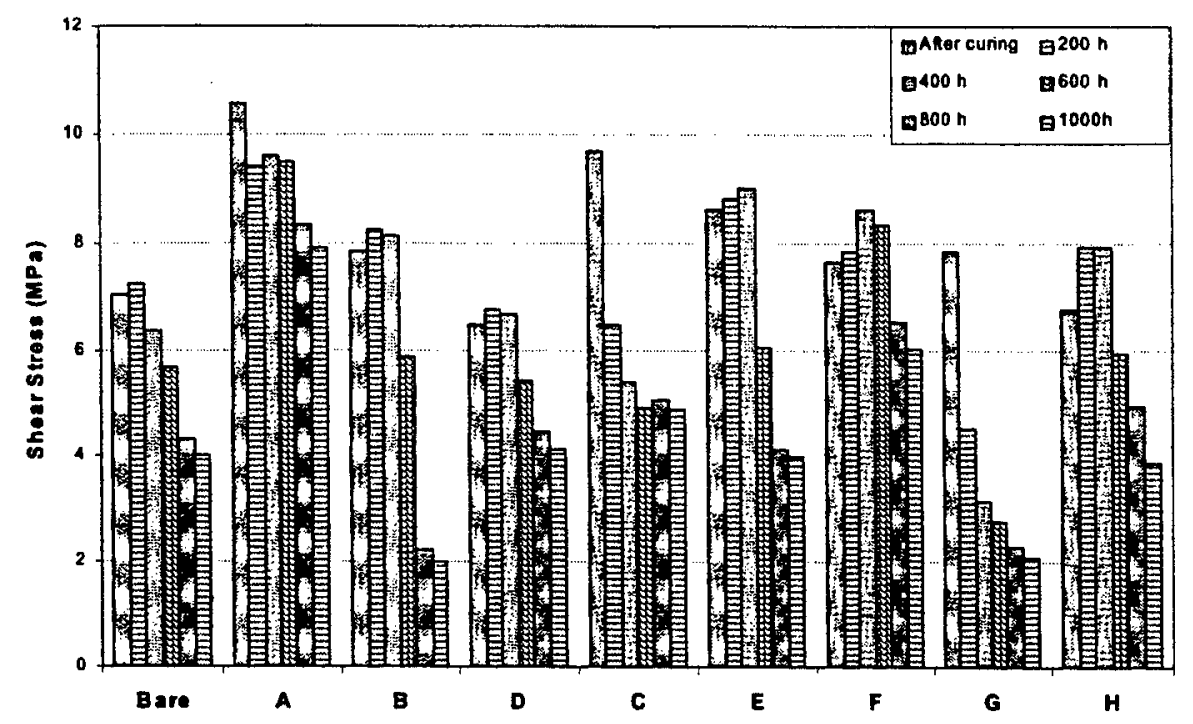

Figure 8 Shear stress between Parylene C and Dow Coming DC3-6550 elastomer with various silane coupling agents after exposure to $85^{\circ} \mathrm{C} / 85 \% \mathrm{RH}$ stressing 


\title{
Novel Bi-layer Conformal Coatings for MEMS Encapsulation
}

\author{
- Parylene C Coated Silicone Elastomers
}

\author{
Jiali Wu, Randy T. Pike, C.P. Wong \\ School of Materials Science and Engineering \\ $\&$ \\ Packaging Research Center \\ Georgia Institute of Technology \\ Atlanta, GA 30332-0245
}

\begin{abstract}
A flexible, smooth, and low profile conformal coating was developed to realize the encapsulation of a Microelectromechanical System (MEMS) device that will be applied to sense the static pressure on aircraft during real flight testing. The encapsulant should be able to protect the MEMS device and the multi-chip module (MCM) from adverse environmental conditions, i.e. mechanical shock, temperature fluctuation, engine fuel and oil contamination, and moisture/mobile ion permeation. Presently, conventional packaging schemes for electronics cannot satisfy this specific outdoor application, and a new encapsulation combination has been designed in accord with the requirement of Reliability Without Hermeticity (RWOH). A bi-layer structure was selected because of property limitations of a single material. Pliable elastomeric silicones, are typically flexible, water repellant, and abrasion resistant. The silicone encapsulant will be first applied to planarize the MEMS surface and function as durable dielectric insulation, stress-relief, and shock/vibration absorbers over a wide humidity/temperature range. To compensate for the deficiency of silicone on engine fuel/oil contamination, parylene $\mathrm{C}$ is to be deposited afterward. This bi-layer coating can achieve excellent bulk properties, such as moisture and mobile ion barrier resistance, chemical compatibility, and electrical insulation characteristics. However, the poor adhesion of parylene $\mathrm{C}$ to silicone greatly restricts its application. To address this problem, silane coupling agents were used as an adhesion promoter. Significant adhesion improvement was achieved by placing an interlayer silane coupling agent to provide interfacial bonding to the silicone elastomeric surface and the parylene $C$ film. Furthermore, a possible mechanism of adhesion enhancement will also be studied.
\end{abstract}

Key words: Microelectromechanical System (MEMS), multi-chip module, bi-layer conformal coating, silicone elastomer, Parylene $\mathrm{C}$, silane coupling agent, reliability without hermeticity (RWOH).

\section{INTRODUCTION}

Long-term, reliable protection of sensitive circuits and components is becoming more important in many of today's delicate and demanding electronic applications. The need for low profile, flexible, conformal and economical packaging in Microelectromechanical System (MEMS) based modular protection has resulted in a new conformal coating design exhibiting Reliability Without Hermeticity (RWOH). General organic passivation materials such as polyimides, silicones, and epoxies have been used as RWOH encapsulants with reasonable success. However, these organic materials are generally moisture permeable. Moisture diffusion rates through organic polymeric encapsulants can be in minutes, hours, or days. The rate depends on the type of material, the material thickness, and the length of diffusion time. Moisture on the surface of the device, in the presence of mobile ions such as $\mathrm{Na}^{+}, \mathrm{K}^{+}$, and $\mathrm{Cl}^{-}$, will result in device failure. [1] To ensure RWOH, we propose to combine a low moisture permeable silicone elastomer coating with a thin film of Para-X-xylyene (Parylene $C$ ) to ensure long-term protection. The silicone elastomer is coated as a relatively thick layer (approximately $250 \mu \mathrm{m})$, primarily for protection of the delicate circuits and as a planarizing layer for the MEMS device. A thin layer of Parylene C (approximately 15$20 \mu \mathrm{m}$ ) is then deposited on top of the thick silicone elastomer layer. This novel combination provides an outstanding barrier to moisture and mobile ion $\left(\mathrm{Na}^{+}\right.$, $\mathrm{K}^{+}, \mathrm{Cl}^{-}$) permeation and an aerodynamically smooth surface for avionics applications. Furthermore, the low surface energy of Parylene $C$ as a top coating can effectively avoid air borne particles [2] and jet fuel contamination of the under-laying silicone coating. 


\section{Conformal Coating Materials}

Silicones are typical pliable elastomeric materials, which can function as durable dielectric insulation, as barriers against environmental contamination, and as stress relieving shock/vibration absorbers over a very wide humidity/temperature range. In this study, Dow Corning Q4-2817 and Dow Corning Hipec 3-6550 were selected as candidate elastomers, to function explicitly as a top profile planarizing agent.

Parylene is a conformal coating family that has high dielectric strength and excellent chemical resistance. [3] Currently, the parylene family is composed of four types of commercial products: Parylene N, C, D, and F. Unlike conventional liquid coating, parylene coatings are applied by vapor deposition in a vacuum chamber at ambient temperature. This unique method of vapor deposition can form $100 \%$ pinhole-free uniform coverage and has the ability to penetrate and coat very complex topographical geometry. Coating thickness with this deposition process is also easily controlled. In this study, Parylene $\mathrm{C}$ is selected as a suitable encapsulating candidate in terms of moisture permeation resistance, chemical resistance, deposition rate and cost consideration. [4]

Table 1 lists the normalized permeability of gasses through polymer materials. The permeability of Parylene $C$ is significantly lower than almost any other engineering plastic. Moisture permeability resistance of Parylene $C$ is 40 times greater than that of silicone materials. Table 2 lists chemical compatibility results of parylene films by film thickness measurements before and after immersion in different organic solvents for $90 \mathrm{~min}$ at room temperature. Common solvents, especially those with similar chemical structures as parylene, such as xylene, result in the most severe swelling of film thickness, which is still less than $3 \%$ of the original parylene film thickness. Parylene $C$ shows excellent chemical resistance to aliphatic hydrocarbons such as octane, the primary component of engine fuel, only resulting in a swelling of $0.4 \%$.

\section{$\underline{\text { Interfacial Adhesion Promoters }}$}

In addition to material considerations, interfacial adhesion reliability between polyimide passiviated Si-based substrate and silicone elastomer, silicone elastomer and Parylene $\mathrm{C}$ also play an important role in real-life applications. Also, the adhesion at various interfaces of the encapsulated MCM device must resist vibrations and air-stream impact during actual flight test.
Table 1. Normalized permeability of gasses through polymer materials

$\mathrm{H}_{2} \mathrm{O}$ in $\mathrm{cm}^{3} . \mathrm{mm} / \mathrm{m}^{2}$.day.atm. All gasses in g.mm/ $\mathrm{m}^{2}$.day.atm

\begin{tabular}{|c|c|c|c|c|c|}
\hline & $\mathrm{O}_{2}$ & $\mathrm{~N}_{2}$ & $\mathrm{CO}_{2}$ & $\mathrm{H}_{2}$ & $\mathrm{H}_{2} \mathrm{O}$ \\
\hline Parylene C & 2.8 & 0.4 & 3 & 43 & 0.08 \\
\hline Parylenc D & 12 & 1.8 & 5 & 94 & 0.1 \\
\hline Parylere N & 15 & 3 & 84 & 213 & 0.6 \\
\hline HDPE & 73 & 17 & 228 & n.d. & 0.12 \\
\hline PS & 138 & 23 & 400 & n.d. & 3.5 \\
\hline PTFE & 223 & 133 & n.d. & 516 & n.d. \\
\hline LDPE & 140 & 80 & 700 & n.d. & 0.6 \\
\hline PC & 124 & 22 & 827 & n.d. & 1.5 \\
\hline FEP & 295 & 126 & 657 & 381 & 0.16 \\
\hline Silicone & 19000 & n.d. & 118000 & 17000 & 3 \\
\hline
\end{tabular}

Table 2. Swelling caused by organic solvents at room temperature

\begin{tabular}{|l|l|c|c|c|}
\hline \multicolumn{2}{|c|}{ Solvents } & \multicolumn{2}{c|}{$\%$ Swelling Parylene } \\
\hline Class & \multicolumn{1}{|c|}{ Test Merriber } & N & C & D \\
\hline Alcohol & Isopropyl & 0.3 & 0.1 & 0.1 \\
\hline Aliphatic Hydrocarbon & iso-Octane & 0.2 & 0.4 & 0.3 \\
\hline Amines & Pyridene & 0.2 & 0.5 & 0.5 \\
\hline Aromatic Hydrocarbon & Xylene (mixed) & 1.4 & 2.3 & 1.1 \\
\hline Chlorinated Alqhatic & Trichloroethylene & 0.5 & 0.8 & 0.8 \\
\hline Chlorinated Aromatic & Chlorobeneze & 1.1 & 1.5 & 1.5 \\
\hline Chlorinated Aromatic & O-Dichlorobeneze & 0.2 & 3.0 & 1.8 \\
\hline "Freon" & Trichlorotrifluoroethane & 0.2 & 0.2 & 0.2 \\
\hline Ketone & Acetone & 0.3 & 0.9 & 0.4 \\
\hline Keton & 2.4-Pentanedione & 0.6 & 1.2 & 1.4 \\
\hline
\end{tabular}

* Film strips were immersed in the test liquids for 90 minutes at room temperature and the thickness was re-measured by the IR method. In every case, equilibrium (no further thickness change) was reached before 90 minutes.

Generally speaking, adhesion theory includes factors such as wet-out phenomena, weak boundary layers, polar adsorption, surface energies, and other effects. The predominant contributor to adhesion, however, is chemical bonding such as that introduced by organofunctional silane "coupling agents". These materials are used in very low concentration, but produce disproportionately large improvements in adhesion performance. Processing and fabrication conditions, which ensure the opportunity for interaction at both ends of the silane molecule, are essential to provide bonding on both sides of the interface. $[5,6]$ In this 
study, a total of 9 different functional silane coupling agents from Dow Coming Co. and Witco Corporation were used as candidate adhesion promoters. An appropriate mechanism is presented later in this study.

Since this is a long-term research project, reliability characterization and evaluation of the conformal coatings is still under study. This paper describes a preliminary aspect of the initial working results and reports a new bi-layer conformal coating design for similar potential applications.

\section{EXPERIMENTAL}

\section{A. SIR Test Vehicle and Conformal Coating Material Selection}

All the conformal coating materials applied in this project were initially evaluated by Surface Insulation Resistance (SIR) measurements using an Alpha Metals SIR $\Omega$ meter (Model 300) test instrument with custom copper/FR4 BellCore Y comb test vehicles. The $50.8 \times 50.8 \mathrm{~mm}$ SIR board surface area was coated with candidate materials immediately after a special cleaning process. [7] After encapsulation, the coated test boards were fixed into a Delp. Corp 16 pattern SIR rack. Initial measurements were made to verify that the needle type test pins penetrated the coatings and made contact with the copper comb pattern. Finally, the test fixture and samples were mounted in a Blue $M$ Humid-Flow Combination Temperature and Humidity Cabinet set at $85^{\circ} \mathrm{C} / 85 \% \mathrm{RH}$. All coatings would be continuously exposed to $85^{\circ} \mathrm{C} / 85 \% \mathrm{RH}$ for $1000 \mathrm{~h}$, with 100 volt dc biasing.

The silicone candidates selected for this study were commercially available and the selection of coating materials was initially based on thermomechanical properties (surviving temperature cycling from $-55^{\circ} \mathrm{C}$ to $125^{\circ} \mathrm{C}$ ) and level of mobile ionic impurities ( $<20$ ppm each of $\mathrm{Cl}^{-}, \mathrm{Na}^{+}$, and $\mathrm{K}^{+}$). All the candidate coatings experienced SIR measurements for further screening.

The two silicone elastomers selected for SIR testing include: 1) Dow Coming Q4-2817 fluorosilicone gel 2) Dow Corning Hipec 3-6550 silicone gel. The moisture and mobile ion resistance of Parylene $C$ with a $5 \mu \mathrm{m}$ coating thickness was also tested by SIR in this study.

\section{B. Reliability Testing}

1) Sample preparation

\section{a. Silicone elastomer coating}

The polyimide passivated Si-based substrates were cleaned using the same method as for SIR board treatment. Within 0.5 hour after cleaning, the silicone materials were uniformly coated on the top of the cleaned substrates using a spin coater (Specialty Coating Systems, Inc. Model 6708D). Curing procedure for the Dow Corning Q4-2817 was $100^{\circ} \mathrm{C}$ for $30 \mathrm{~min}$. While, for Dow Corning Hipec 3-6550 was $150^{\circ} \mathrm{C}$ for $60 \mathrm{~min}$.

\section{b. Silane Coupling Agent Application}

Direct pretreatment is the easiest and most satisfactory method to apply a silane to an inorganic surface. Two methods find extensive use: 1) Aqueous pretreatment, which is primarily used for fiberglass. Coating is accomplished by applying a dilute, aqueous solution of the silane, generally adjusted to $\mathrm{pH} 4$ with acetic acid. The silane hydrolyzes and forms a layer of silanol molecules at the substrate surface. The solution, typically with a concentration of silane within 0.2 to $0.5 \%$, will ordinarily dissipate onto the surface. This method is generally performed with high efficiency. 2) Nonaqueous blending, which is widely used for mineral fillers. The silane, which is undiluted or mixed with a spreading solvent, is added to the inorganic substrate and thoroughly dry blended at low shear to give a uniform coating. Dry blending offers a convenient and effective route to coupling agent treatment of particulate inorganics such as alumina, silica and glass microbeads used as fillers in composites. A silane treatment level of between 0.5 and $1.0 \%$ is appropriate for most common minerals. The additive method is an alternate route commonly used to apply silane coupling agents. With this method, the silane is added to the polymeric phase from which it diffuses or migrates to the inorganic surface. Reaction occurs first by hydrolysis, and then by condensation to produce siloxane coupling linkages. Uniform treatment is dependent upon thorough blending of the mixture and filler. Typically, the silane is added at a concentration of $1.0 \%$ or less.

In regard to our unique bi-layer $\mathrm{RWOH}$ design, the three kinds of silane application methods discussed above were not appropriate since of parylene vapor deposition and silicone swelling in silane solutions. To address this problem, a new silane application method (vapor deposition) was used to directly deposit silane on the surface of the silicone elastomer. This method was selected to avoid film swelling and still produce a uniform adhesion promoter layer. Consequently, Parylene $C$ is 
promptly polymerized on top of the silane for fear of surface contamination, and ensure that the silane still remains on the surface of the silicone layer.

Prior to silane application, all the silicone coated substrates were treated with UV-ozone at $50^{\circ} \mathrm{C}$ for $5 \mathrm{~min}$. Immediately following treatment, the side with the silicone elastomer was exposed to the silane solutions. Silane solutions were then heated between $60^{\circ} \mathrm{C}$ to $70^{\circ} \mathrm{C}$ for $20 \mathrm{~min}$ to vaporize sufficient silane, which was uniformly condensed on the entire surface of the silicone elastomers. An excess of silane will lead to a swelling in the silicone coating. After that, the samples were immediately placed into a Parylene Deposition System, Lab Coater 2010, from Special Coating System Co. for Parylene C deposition.

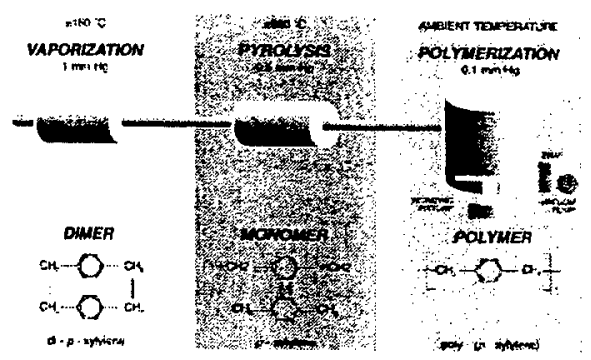

Figure 1. Schematic graph of Parylene C deposition process

\section{c. Parylene C Deposition}

Parylene $\mathrm{C}$ deposition was conducted using the Gorham Method. The Parylene $C$ coating process starts with the vaporization of the dimer at $150^{\circ} \mathrm{C}$, 0.1 Torr. The resulting dimer gas is further elevated at $650^{\circ} \mathrm{C}$ in the pyrolysis zone to obtain two methylene bonds to generate a stable para-xylylene. Finally, the monomer is sent to the ambient temperature deposition vacuum chamber where the para-xylene polymerizes and coats on the surface of the silicone elastomers. [8] A Schematic graph of the parylene C deposition process is illustrated in Figure 1.

\section{2) Reliability Testing}

All the single layer or bilayer shear strength test samples were exposed to $85^{\circ} \mathrm{C} / 85 \% \mathrm{RH}$ (Relative Humidity) chamber (Blue $\mathrm{M}$, model No. AC7602HA) for $1000 \mathrm{~h}$ and Thermal Shock chamber (Thermotron Environmental Test Chamber, ATS320-DD-10-705-LN2) for 500cycles. Thermal shock cycling was set from $-55^{\circ} \mathrm{C}$ to $125^{\circ} \mathrm{C}$, temperature ramp time was $15 \mathrm{~min}$, and the dwell period at either $55^{\circ} \mathrm{C}$ or $125^{\circ} \mathrm{C}$ was $15 \mathrm{~min}$. The adhesion test interval for $85^{\circ} \mathrm{C} / 85 \% \mathrm{RH}$ aging was $200 \mathrm{~h}$, and for thermal shock stressing was 100 cycles.

\section{RESULTS AND DISCUSSION}

SIR measurement results in Figure 2 indicate that the Dow Corning Hipec 3-6550, Q4-2817 and Parylene $\mathrm{C}$ conformal coatings pass the aerospace specification limit of 1E08 Ohms. The surface insulation resistance shows a slowly decreasing trend during the testing period. This can be attributed to moisture and mobile ion ingress facilitating mobile ion migration from cathode to anode during biasing, which will result in resistance decrease.

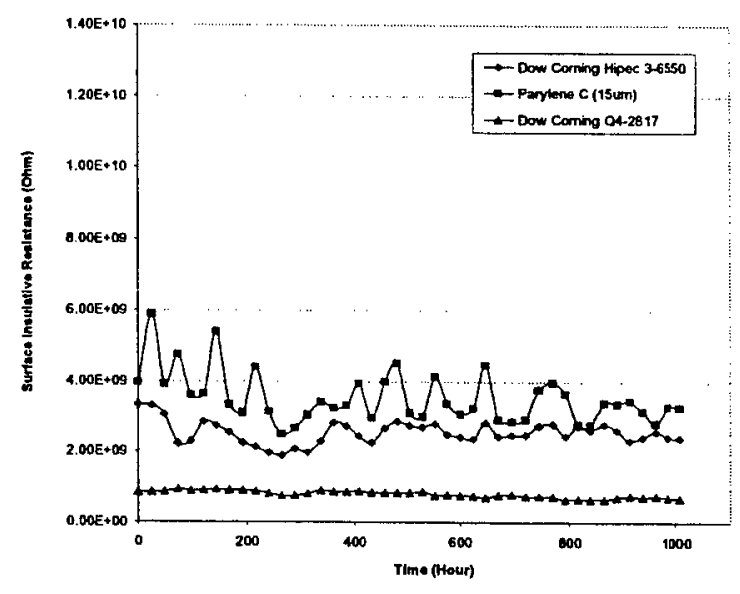

Figure 2. Surface insulative resistance of Parylene C and silicone elastomers varied with time under 100volt de biasing

To improve the adhesion strength between the silicone elastomer and Parylene $\mathrm{C}$, nine different functional silane coupling agents were selected as adhesion promoter candidates between Parylene $\mathrm{C}$ and Silicone Elastomer based on chemical covalent relation between silane organic functional groups and Parylene $\mathrm{C}$ chemical structure. Chemical structures of all nine silane coupling agents are listed in Table 3.

Shear strengths between Parylene $\mathrm{C}$ and Dow Corning Q4-2817 after sample preparation using various silane coupling agents as adhesion promoters are illustrated in Figure 3. Results indicate that shear strengths obviously increase using silane coupling agents $C, D$ and $H$, and shear strengths decrease using silane coupling agents $A, F$ and $G$. Shear strengths greatly decrease using silane coupling agent 
Table 3. Candidate Silane Coupling Agents for Enhancing Adhesion

\begin{tabular}{|c|c|c|}
\hline Designation & Chemical Name & Chemical Formula \\
\hline$A-174$ & $\begin{array}{l}\gamma-\text { M ethacryloxypropyl } \\
\text { trimethoxysilane }\end{array}$ & 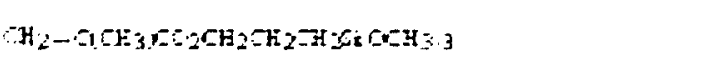 \\
\hline$A \cdot 1120$ & $\begin{array}{l}N-\beta \cdot(\text { A minoethy })-\gamma-a \operatorname{mino} \\
\text { propyltrimethoxysilane }\end{array}$ & 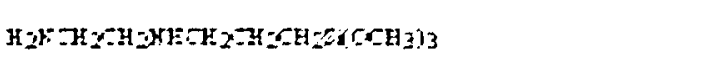 \\
\hline PSX554 & & 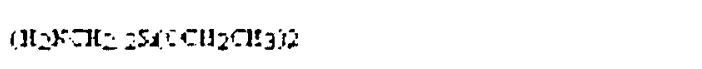 \\
\hline Y -9669 & $\begin{array}{l}\text { N-Phenyl-y-am in opropyl } \\
\text { trimethoxy silane }\end{array}$ & is \\
\hline A -1102 & $\gamma$-A minopropyltriethy lsilane & 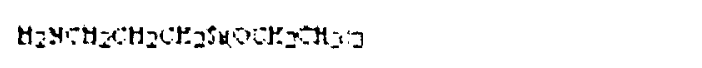 \\
\hline$A-171$ & Vinyltrimethoxysilane & $C H=C H S$ SWITS \\
\hline A-151 & Vinyltriethoxysilane & 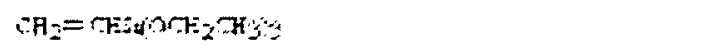 \\
\hline A -172 & Vinyl-tris-(2-methox yethax $y)$ sil & $\mathrm{H}_{2}=\mathrm{CH}_{2} \mathrm{OH}_{2} \mathrm{H} \mathrm{H}_{2} \mathrm{OH}_{2}$ \\
\hline A -2120 & $\begin{array}{l}N-\beta-(\text { aminoethyl })-\gamma \text {-aminopropyl } \\
\text { methyldimethoxysilane }\end{array}$ & 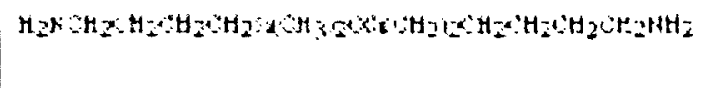 \\
\hline
\end{tabular}

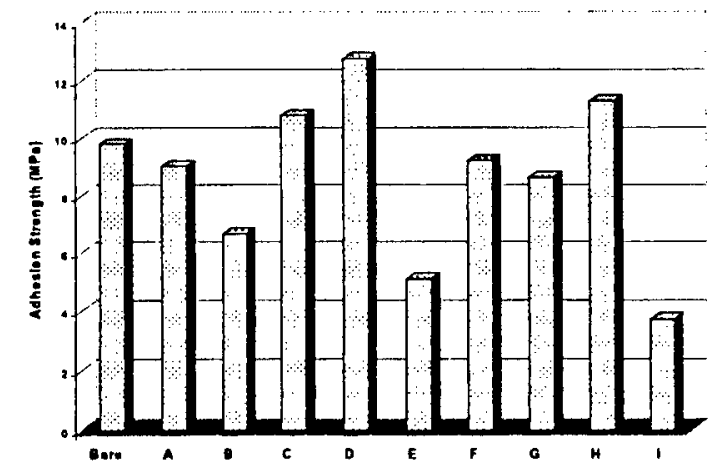

Figure 3. Shear strength between Parylene $C$ and Dow Coming Q4-2817 elastomer with various silane coupling agents after sample preparation

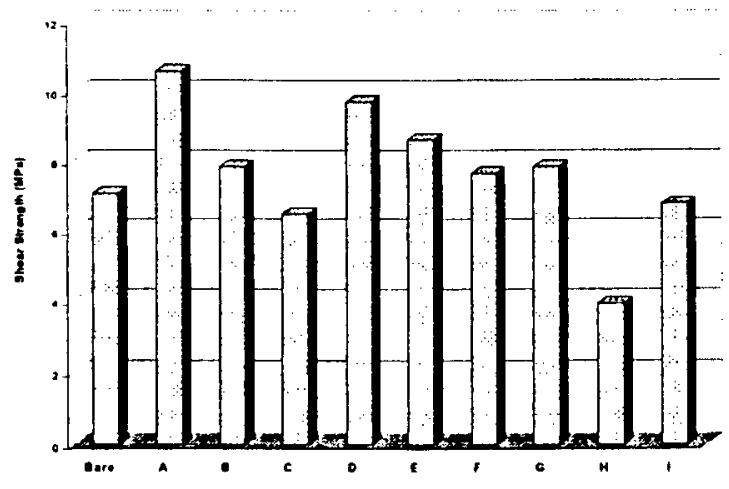

Figure 4. Shear strength between Parylene $C$ and Dow Corning Hipec 3-6550 elastomer with various silane coupling agents after sample preparation 
$\mathrm{B}, \mathrm{E}$, and $\mathrm{I}$. Therefore, samples with silane coupling agents $A, C, D, F, G$, and $H$ require further reliability testing. Shear strengths between Parylene $C$ and Dow Coming Hipec 3-2817 after sample preparation are shown in Figure 4. Results indicate that shear strengths increase using silane coupling agents $\mathrm{A}, \mathrm{B}$, $D, E, F$, and $G$. Shear strengths decrease using silane coupling agents $\mathrm{C}$ and $\mathrm{I}$, and shear strengths greatly decrease using silane coupling agent $H$. Subsequently, samples with silane coupling agents $\mathrm{A}$, $\mathrm{B}, \mathrm{C}, \mathrm{D}, \mathrm{E}, \mathrm{F}, \mathrm{G}$, and I will be subjected to further reliability testing.

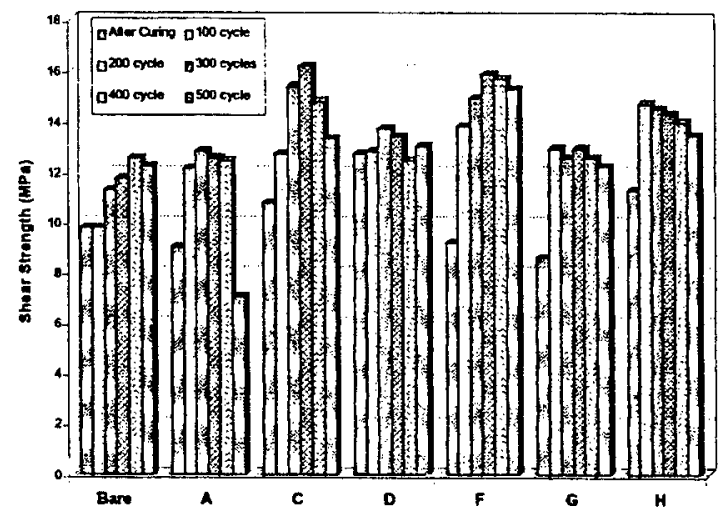

Figure 5. Shear strength between Parylene $C$ and Dow Coming Q4-2817 elastomer with various silane coupling agents after exposure to thermal shock aging

Shear strength results of samples submitted to thermal shock cycling are shown in Figure 5 at 100 cycle intervals. Shear strength results between Parylene $\mathrm{C}$ and Dow Coring Q4-2817 after $85^{\circ} \mathrm{C} / 85 \%$ RH aging are shown in Figure 6 at 200 hour intervals. From Figure 6 it is observed that the shear strength of most samples increases after exposure to thermal shock cycling for 100 cycles. The possible reason for this is that the intrinsic cross-linking reaction of the silicone elastomer is maintained under thermal shock cycling during this period, and some cyclic oligomers, such as D3, D4 and D5, etc. are continuously diffused out. As a result, the modulus increase of the silicone elastomer leads to a shear strength increase. However, after sample exposure to thermal cycling for a considerable time, CTE mismatch between Parylene C (35-40ppm) and silicone elastomer (300-500 ppm) intensified and causes microdelamination to occur at some interfacial

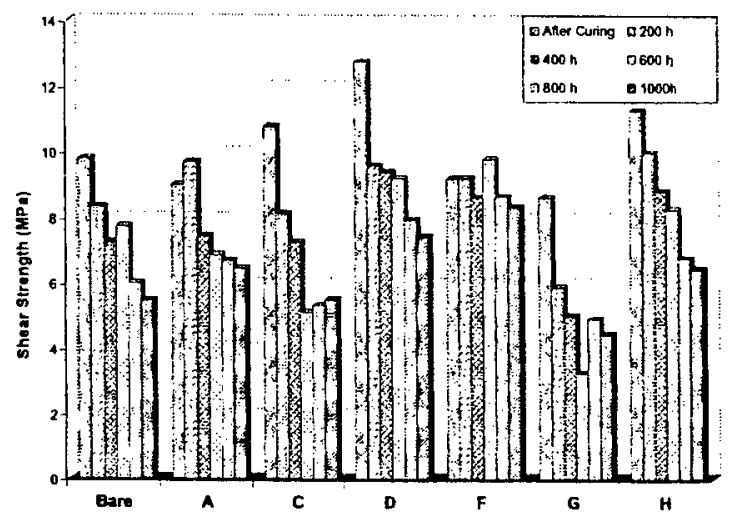

Figure 6. Shear strength between Parylene $\mathrm{C}$ and Dow Corning Q4-2817 elastomer with various silane coupling agents after exposure to $85^{\circ} \mathrm{C} / 85 \% \mathrm{RH}$ stressing

sites, which finally become the failure centers during shear testing and lower the interfacial adhesion. However, shear strengths for most samples continuously decrease under $85^{\circ} \mathrm{C} / 85 \% \mathrm{RH}$ aging condition due to the modulus of silicone elastomer reduction. This situation may be the result of two factors. One possible reason is that when the bilayer sample is kept under $85^{\circ} \mathrm{C}$ for an extended time, some low molecular silicone cyclics may be continuously generated and trapped in the cross-linked network as plasticizers, since the high moisture pressure intuitively prohibits the cyclics outward diffusion. Another possible reason is that the moisture may cause the siloxane to inversely hydrolyze into silanols at $85^{\circ} \mathrm{C}$. The moisture came from permeation of moisture in $85 \% \mathrm{RH}$ atmosphere through the Parylene $\mathrm{C}$ sheared (removed) areas during prior die shear testing.

From result analysis of Figure 5 and Figure 6, it can be concluded that the best adhesion promoters for the Parylene $C$ and Dow Corning Q4-2817 combination are silane coupling agents $\mathrm{D}$ and $\mathrm{F}$.

Shear strength results of the Parylene $\mathrm{C}$ and Hipec 3-6550 bi-layer combination after different intervals of thermal shock aging and $85^{\circ} \mathrm{C} / 85 \% \mathrm{RH}$ stressing are shown in Figure 7 and Figure 8, respectively. The shear strength trends are the same as those between Parylene C and Dow Corning Q4-2817. It can be observed that the best two silane coupling agents for Parylene $C$ and Dow Corning Hipec 3-6550 are silane coupling agent $A$ and $F$ as confirmed by adhesion reliability testing. 


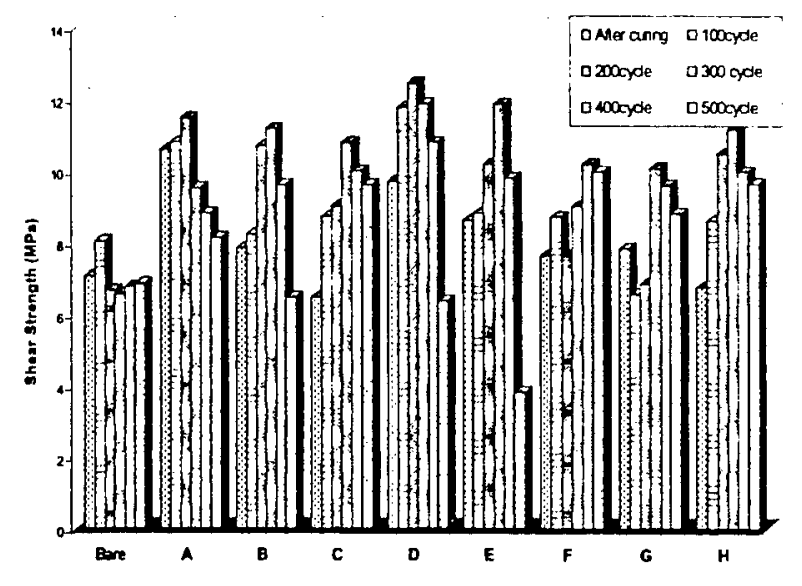

Figure 7. Shear strength between Parylene $\mathrm{C}$ and Dow Corning Hipec 3-6550 elastomer with various silane coupling agents after exposure to thermal shock aging

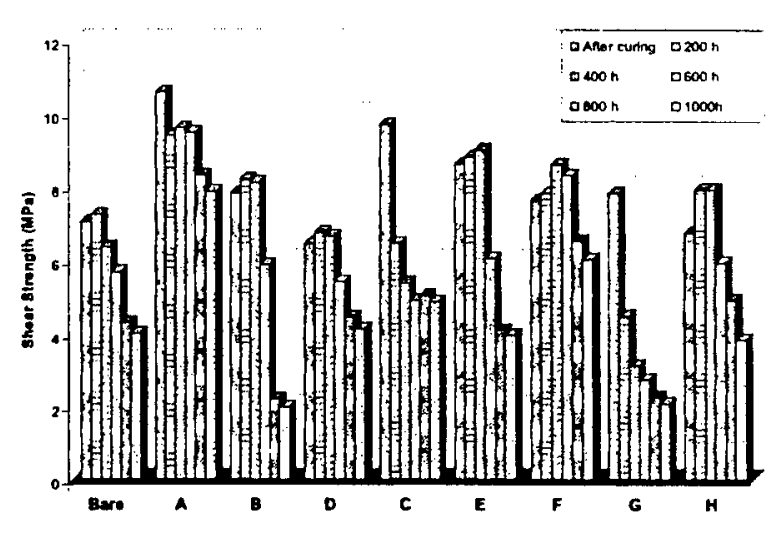

Figure 8. Shear strength between Parylene $\mathrm{C}$ and Dow Corning Hipec 3-6550 elastomer with various silane coupling agents after exposure to $85^{\circ} \mathrm{C} / 85 \% \mathrm{RH}$ stressing

\section{CONCLUSION}

A novel bilayer conformal coating for MEMS device encapsulation has been designed in accordance with the requirement of RWOH. Parylene $\mathrm{C}$ is a promising conformal coating for aerospace application. Adhesion between Parylene $\mathrm{C}$ and silicone elastomer has been intensified by applying silane coupling agents at the interface as adhesion promoters. The systems reliability has also been investigated by die shear testing after thermal shock stressing and $85^{\circ} \mathrm{C} / 85 \% \mathrm{RH}$ aging. Test results indicate that the silane species with a vinyl or anilino group at one end and a methoxy group at the other end will show the best adhesion enhancement. A proper mechanism will been proposed in recent study.

\section{ACKNOWLEDGEMENT}

The financial support from the Boeing Company and Specialty Coating Systems, Inc. is greatly appreciated.

\section{REFERENCES}

[1] C.P.Wong, Ed. "Polymers for Electronic and Photonic Applications", Academic Press, San Diego, CA (1993).

[2] US Patent, No. 5075174, "Parylene Coated Elastomers". (Dec. 24, 1991).

[3] Roger Olson, "Parylene Conformal Coating and It's Applications for Electronics", Proceedings of the Symposium on the Interface, Published by Interface Foundation of North America, Fairfax Station, VA USA, p 112 (1991).

[4] Parylene technical data web site: http://ww'w.paratronix.com/tdata/.

[5] E.P. Plueddemann, "Adhesion Through Silane Coupling Agents", Proceeding of the $25^{\text {th }}$ Annual SPI PR/C Conference, Section 13-D, pl (1970).

[6] P.E. Cassidy, and W.J. Yager, "A Review of Coupling Agents as Adhesion Promoters", NASA contract NAS8-24073, TRACOR Document Number T-70-AU-7129-U, p13 (1987)

[7] C.P. Wong and R. McBride, "Preencapsulation Cleaning Methods and Control for Microelectronics Packaging", IEEE Transaction on CMPT, Part A, Vol. 17-4, p 542 (1994).

[8] L. You, G. R. Yang, C.I. Lang, P. Wu, and J.A. Moore, "Vapor deposition of parylene films from precursors", Proceedings of the 3rd Biennial Meeting of Chemical Perspectives of Microelectronic Materials, Boston, MA, USA (1992). 


\title{
Evaluation and Characterization of Reliable Non-Hermetic Conformal Coatings for Microelectromechanical System (MEMS) Device Encapsulation
}

\author{
Jiali Wu, Member, IEEE, Randy T. Pike, C. P. Wong, Fellow, IEEE, Namsoo P. Kim, and Minas H. Tanielian
}

\begin{abstract}
The thrust of this project was to evaluate commercial conformal encapsulation candidates for low cost aerospace applications. The candidate conformal coatings evaluated in this study included silicone elastomers, epoxies, and Parylenes with bi-layer or tri-layer designs. Properties characterized in this study included mobile ion permeation and moisture ingress resistance, interfacial adhesion variation through thermal shock cycling and $85^{\circ} \mathrm{C} / 85 \%$ RH aging. Surface Insulation Resistance (SIR), Triple Track Resistance (TTR) and die shear strength were used for the corresponding electrical and physical property characterizations. Parylene $\mathrm{F}$ displayed excellent properties for environmental protection. Silicone elastomers displayed less resistance to the harsh environment as compared to the Parylene family (N, C, D types), but it could provide advantages for low residual stress applications. The change in adhesion strength between Parylene $C$ and silicone elastomers after exposure to thermal shock cycling or $85^{\circ} \mathrm{C} / 85 \% \mathrm{RH}$ aging for different time periods were conducted from die shear test in terms of the interfacial failure. SIR values of all the candidate materials after $1000 \mathrm{~h}$ exposure to $85^{\circ} \mathrm{C} / 85 \% \mathrm{RH}$, with $100 \mathrm{~V}$ dc for resistance measurement, range from $1 \times 10^{8}-1 \times 10^{9} \Omega$. Leakage current values after $1000 \mathrm{~h}$ exposure to $85^{\circ} \mathrm{C} / 85 \% \mathrm{RH}, 175 \mathrm{~V}$ bias, are in the range of $10^{-9}$ to $10^{-11} \mathrm{Amp}$. The bi- or tri-layer conformal coating combination investigated in this study showed significant promise for encapsulation of the microelectromechanical system (MEMS) devices.
\end{abstract}

Index Terms-Conformal coatings, leakage current, mobile ion permeation, moisture ingress, surface insulation resistance.

\section{INTRODUCTION}

$\mathbf{R}$ ELIABLE nonhermetic conformal encapsulants have been widely applied since the 1970s [1]. Recent applications have been aimed at the automotive, communication and aerospace markets. The thrust of this study is to searching for a high performance nonhermetic conformal coating, which can protect the microelectromechanical system (MEMS) devices from adverse environment as well as mechanical stresses from internal or external due to the encapsulation process and real application. A primary focus to ensure the MEMS device

Manuscript received August 23, 1999; revised July 26, 2000. This work was supported in part by DARPA.

J. Wu, R. T. Pike, and C. P. Wong are with the School of Materials Science and Engineering, Packaging Research Center, Georgia Institute of Technology, Atlanta, GA 30332-0245 USA.

N. Kim and M. Tanielian are with The Boeing Company, Seattle, WA 98124-2207 USA.

Publisher Item Identifier S 1521-3323(00)09465-X. operating reliability exposed to hostile environments is to use multilayer conformal coatings that exhibit complementary merits to meet the stringent requirements. Historically, reliable nonhermetic encapsulation materials are: high purity epoxies, room temperature vulcanized (RTV) silicones, fluorinated silicone based materials, fluorinated acrylics, polyurethane, Parylenes (poly para-xylyenes Types N, C, D, F), BCB and polyimides [2]-[10], []These materials can meet a broad variety of different aspects of environmental protection criteria.

The key specifications established by the aerospace industry for the prototype MEMS device required that the candidate commercial encapsulants should display excellent resistance to mobile ion permeation, high humidity, and constant thermal and mechanical properties within wide temperature extremes $\left(-55^{\circ} \mathrm{C}-100^{\circ} \mathrm{C}\right)$. Low internal stress of the coating is also importance for guaranteeing the accurate static force sense of the piezopressure sensor. Additionally, a low profile $(<2 \mathrm{~mm})$ was necessary to ensure a proper fit for the device.

Typically, conformal coating applying methods include glob-top coating, spray coating, curtain coating, spin coating, and chemical vapor deposition (CVD). And one of the key issues for achieving high reliability with nonhermetic conformal encapsulants for microelectronic applications is pre-encapsulation cleaning [2]. Qualified pre-cleaning of the electric circuits can perform the best interfacial adhesion. The cleaning procedure adopted in this study based on the method developed by Wong and McBride [11].

Candidate nonhermetic conformal coatings exhibit chemical compatibility, an appropriate curing temperature, low residual stress, good adhesion, and good solvent resistance. A historical overview indicated that encapsulants meeting our targeted environmental protection requirements could be silicone elastomers and gels, epoxies, and poly-para-xylylenes (Type $\mathrm{C}$ and F) available from commercial market. However, their environmental protection performance for our particular application was not previously reported in the literatures. Therefore, it was our aim to select the high promising candidates and evaluate and characterize their required properties for MEMS device encapsulation.

Conformal coating candidate materials employed in this study include commercial epoxy resins, silicone elastomers and gels, Parylenes $\mathrm{C}$ and $\mathrm{F}$ type, and fluoroacrylics. Detailed test sample preparation, test setup and vehicle's configuration as well as series results will be presented in the following. 


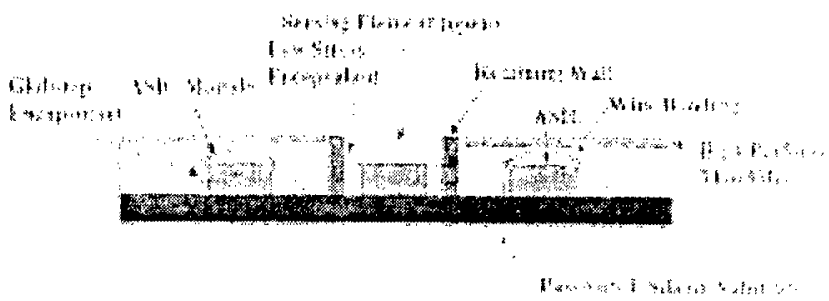

Fig. 1. Complex topographical schematic of prototype MEMS device.

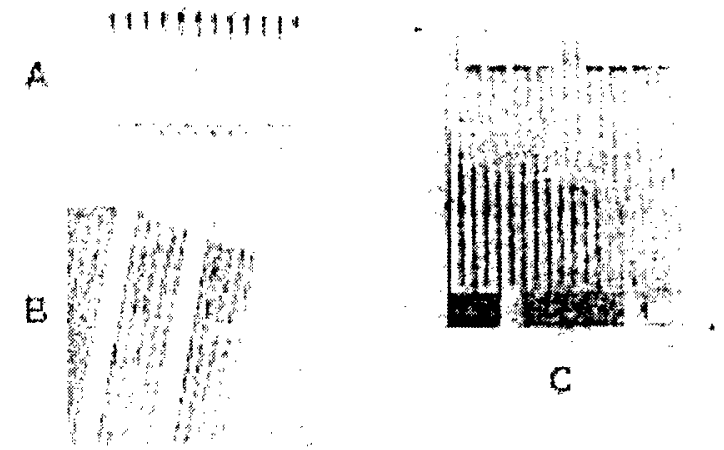

Fig. 2. Sample test vehicles for environmental testing. (a) Triple track resistor test vehicle--TiPdAu metallization $+75 \mu \mathrm{m}$ spacing. (b) Scanning electron microscope (SEM) picture of metal tracks of triple track test vehicle. (c) Modified Bellcore $Y$ comb pattern-Cu metallization $+2 \mathrm{~mm}$ spacing.

\section{EXPERIMENTAL}

\section{A. Prototype MEMS Device}

The design specifications of the prototype MEMS device consisted of a $20.32 \mathrm{~mm} \times 20.32 \mathrm{~mm}$ (equals to 0.8 in $\times 0.8$ in) square multilayer structure with a complex topographical surface (Fig. 1). The base substrate was polyimide passivated silicon. Copper metallization was employed by an MCM-D process. Discrete devices, analog and digital application specific integrated circuits (ASICs) were integrated onto the substrate with a pick-and-place approach. Interconnection was accomplished with a variety of methods including wire bonding, beam lead attach, electrically conductive adhesives, and tape automated bonding (TAB).

\section{B. Moisture Ingress, Mobile Ion Permeation, and Adhesion Test Vehicles}

In order to determine the conformal coating environmental protection, resistance to moisture ingress and mobile ion permeation into the prototype device, unique test vehicles were selected to characterize the encapsulants nonhermetic sealing properties (Fig. 2). Fig. 2(a) is the triple track test (TTT) vehicle used for monitor resistance to moisture ingress and mobile ion permeation of the coatings. It consists of a set of three meandering parallel conductor lines, which are made of $\mathrm{Ta}_{2} \mathrm{~N}$ metallization with $75 \mu \mathrm{m}$ line spacing. And TiPdAu contacts metallized on an $\mathrm{Al}_{2} \mathrm{O}_{3}$ substrate. Fig. 2(b) is a SEM image of metal tracks of TTT vehicle.

The test vehicle used for evaluating surface insulation resistance [Fig. 2(c)] was modified Bellcore Y SIR test board. These vehicles consisted of a $80 \mathrm{~mm} \times 80 \mathrm{~mm}$ (equals to $2 i n \times 2 i n$ ) FR4 substrate with a five-probe pad copper metallized comb pattern with $2 \mathrm{~mm}$ line separation.

Die shear adhesion testing was conducted with $25.4 \mathrm{~mm} \times$ $25.4 \mathrm{~mm}$ (equals to $1 \mathrm{in} \times 1 \mathrm{in}$ ) monolayer and bilayer conformally coated test vehicles as substrate and $2 \mathrm{~mm} \times 2 \mathrm{~mm}$ (equals to $80 \mathrm{mil} \times 80 \mathrm{mil})$ silicon die as tiles. These vehicles consist of a sandwich test structure that includes a substrate of polyimide passivated silicon, a middle layer of the conformal coating materials (silicone, or silicone and parylene $\mathrm{C}$, or epoxy and silicone) and a top layer of tiles passiviated with polyimide. Each die shear adhesion data was an average of ten samples.

\section{Pre-encapsulation Cleaning Technique}

The preparation of silicon substrates, tiles, SIR and TTT test vehicles included the following sequential steps to insure cleanliness:

1) 5 min soak in Bioact ${ }^{\circledR}$ EC-7R ${ }^{\mathrm{TM}}$ terpene organic solvent;

2) 5 min soak in terpene during ultrasonic cleaning;

3) 5 min soak in isopropyl alcohol;

4) $5 \mathrm{~min}$ soak in isopropyl alcohol during ultrasonic cleaning;

5) 5 min soak in distilled water during ultrasonic cleaning;

6) 3 time rinses with distilled water;

7) 2 min soak in 50 ppm surfactant;

8) 5 min rinse with distilled water;

9) $120^{\circ} \mathrm{C}$ bake at $28 \mathrm{~mm} \mathrm{Hg}$ for $30 \mathrm{~min}$;

10) UV-Ozone treatment at $50^{\circ} \mathrm{C}$ for $5 \mathrm{~min}$ with a Samco Model UV-1 dry stripper.

\section{Test Vehicle Encapsulation}

Liquid epoxy and silicone coatings were spin coated on substrates with a Specialty Coating Systems (SCS), Model P6708D coater. Parylene C and Parylene F films were deposited by the Gorham Method. Parylene C films were deposited with a SCS model PDS 2010 coater. Parylene F films were deposited with a unique vacuum deposition system at SCS. The candidate commercial encapsulants evaluated in this study were cured according to manufacturer specifications.

FP4450 and FP4460 are single component thermal cure epoxy resins supplied by Dexter Hysol. These epoxies were cured in air at $150^{\circ} \mathrm{C}$ for $30 \mathrm{~min}$.

Q1-4939 and DC1-4207 are two components (base resin and curing agent) thermal cure silicone encapsulants supplied by Dow Corning. Q1-4939 encapsulated samples were cured in air at $150{ }^{\circ} \mathrm{C}$ for $60 \mathrm{~min}$. DC1-4207 samples were cured at $60^{\circ} \mathrm{C}$ for $30 \mathrm{~min}$.

$D C 1-2577, D C 1-2620, D C 1-3140$, and $D C 1-3145$ Clear are single component room temperature vulcanizing silicone encapsulants supplied by Dow Corning. Curing of these coatings was accelerated in air at $100^{\circ} \mathrm{C}$ for $30 \mathrm{~min}$.

Parylene $C$ (poly (chloro-para-xylylene)) and Parylene $F$ (poly (difluoro-xylylene)) were originally developed by Union Carbide. These materials were conformally coated onto test vehicles at Specialty Coating Systems, Inc. using a three-step vacuum deposition process. 
Fluorad FC-720 and $F C-725$ are fluoroacrylic conformal coatings in butyl acetate supplied by $3 \mathrm{M}$ Specialty Chemicals Division. The coatings were cured at $100^{\circ} \mathrm{C}$ for $30 \mathrm{~min}$.

\section{E. Environmental Stressing}

Device operating conditions were simulated with temperature humidity aging and thermal shock cycling. Temperature humidity aging was conducted at $85^{\circ} \mathrm{C} / 85 \% \mathrm{RH}$ with $175 \mathrm{~V}$ bias for $1000 \mathrm{~h}$ in a Blue $\mathrm{M}$ Humid-Flow combination temperature and humidity cabinet. Thermal shock cycling was performed with a Blue M Model test chamber with $1 \mathrm{~h}$ temperature cycling from $-55^{\circ} \mathrm{C}$ to $125^{\circ} \mathrm{C}$ with $15 \mathrm{~min}$ dwelling at each temperature extreme (Mil. Spec 1014, Step B) for a total of 500 cycle.

\section{F. Die Shear Testing}

Die shear measurements were performed with a Royce Instruments System $552,100 \mathrm{~K}$ adhesion analyzer. Test vehicles subjected to $85^{\circ} \mathrm{C} / 85 \% \mathrm{RH}$ for accelerating aging first and then were measured every $200 \mathrm{~h}$ for a total duration of $1000 \mathrm{~h}$. Samples subjected to thermal shock cycling were measured every 100 cycle for a total of 500 cycle.

\section{G. Surface Insulation Resistance and Leakage Current Measurements}

To characterize the conformal coating resistance to moisture ingress and mobile ion permeation, TTT and SIR test vehicles were used. In-situ SIR measurements were conducted with an Alpha Metals, Model 300 Sirometer, at $85^{\circ} \mathrm{C} / 85 \% \mathrm{RH}$ with $100 \mathrm{~V} \mathrm{dc}$ for resistance measurement for $1000 \mathrm{~h}$. In-situ leakage current and resistance measurements were recorded at $85^{\circ} \mathrm{C} / 85 \% \mathrm{RH}, 175 \mathrm{~V}$ dc bias, for $500 \mathrm{~h}$ with a Keithley Model 82005 multiplexer, Hewlett Packard 34401A multimeter, Hewlett Packard Harrison 6207B DC Power Supply, Keithley Model 616 Electrometer, Dana 4700 Multimeter, and National Instruments LabView 5.0 software for automation.

\section{RESULTS AND DISCUSSION}

The initial selection of an effective protective coating for the prototype MEMS device was focused on identifying two candidate material/encapsulants. The first candidate encapsulant was applied to the surface of the epoxy encapsulated ASICs to provide environmental protection and a planar surface of the microelectronic devices and interconnects, at the same time the internal stress caused by the coating will not affect the accurate measurement of the piezoelectric pressure sensor. The second candidate encapsulant was deposited in the central part of the device to protect the sensing element and beam leads from the corrosion due to the mobile ion permeation and moisture ingress. This candidate encapsulant required extremely low stress to prevent the device and beam lead shifting during operation.

A survey conducted to identify potential commercially available candidates indicated low stress silicones have historically shown good performance in harsh and hostile environments. Furthermore, a thin film of Parylene deposited on top of the silicone will enhance its organic contamination [12]. Electrical testing methods including SIR and triple track testing methods
TABLE I

SURFACE INSULATIVE RESISTANCE RESULTS $\left(85^{\circ} \mathrm{C} / 85 \% \mathrm{RH}, 100 \mathrm{~V}\right.$ dC APPLIED ON EVERY $6 \mathrm{~h}$ )

\begin{tabular}{|c|c|}
\hline \multicolumn{2}{|c|}{ Selected SIR Data } \\
\hline Material & Average 1000 Hour Resistance (G $\Omega$ \\
\hline DH FP 4450 & 3.61 \\
\hline Dow Coming 3-6550 & 3.0 \\
\hline Parylene C $15 \mu \mathrm{m}$ & 2.85 \\
\hline Dow Coming Q I-4939 (10:1) & .77 \\
\hline $3 \mathrm{M} \mathrm{FC}-725$ & .0064 \\
\hline $3 \mathrm{M} \mathrm{FC}-722$ & .0026 \\
\hline
\end{tabular}

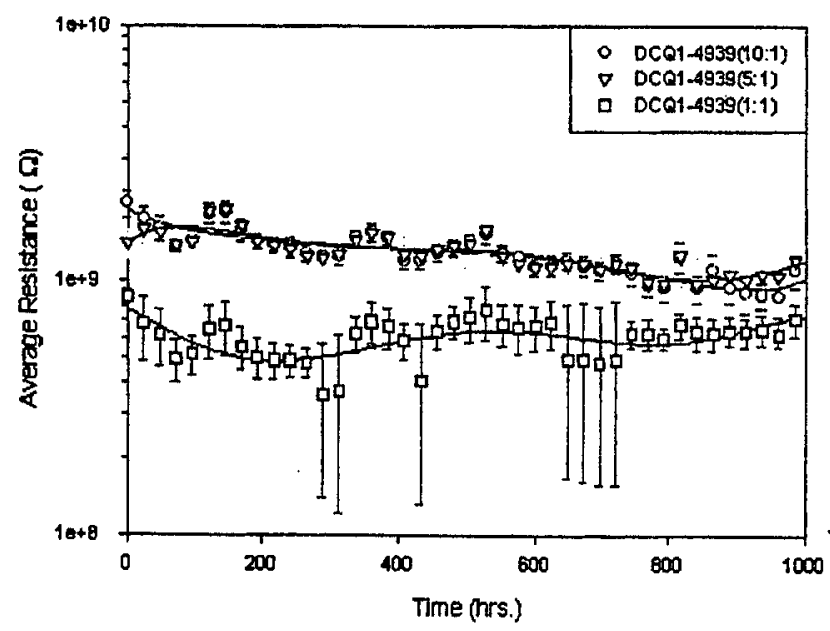

Fig. 3. Surface insulative resistance result for low tress silicone elastomers and gels $\left(85^{\circ} \mathrm{C} / 85 \% \mathrm{RH}, 100 \mathrm{~V}\right.$ dc applied on every $6 \mathrm{~h}$ for resistance measurement)

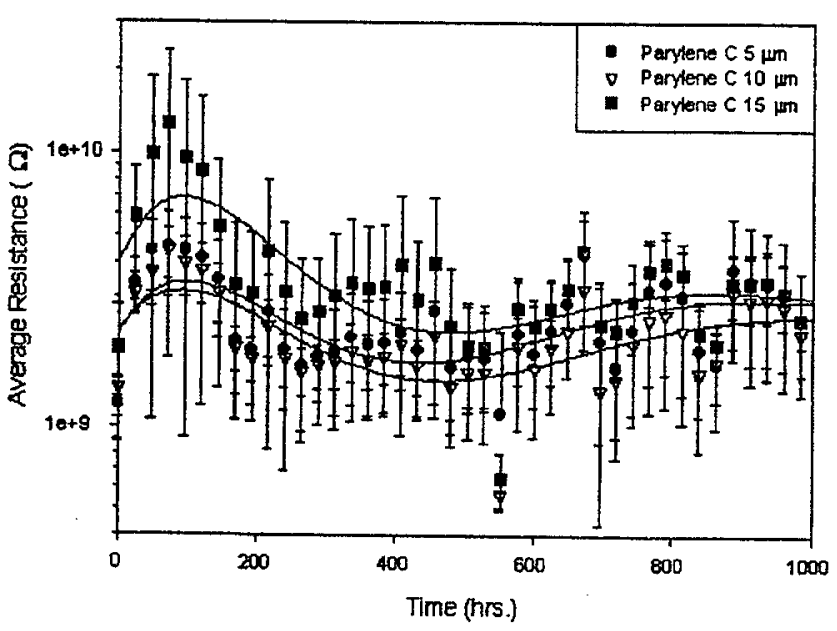

Fig. 4. SIR results for varying film thickness of Parylene $\mathrm{C}\left(85^{\circ} \mathrm{C} / 85 \% \mathrm{RH}\right.$, $100 \mathrm{~V} \mathrm{dc}$ applied on every $6 \mathrm{~h}$ for resistance measurement).

were selected as a fast and efficient screening method for qualifying potential candidate materials.

\section{A. Surface Insulation Resistance (SIR) Measurements}

SIR test was performed in $85^{\circ} \mathrm{C} / 85 \% \mathrm{RH}$ to provide electrical property evaluation for screening candidate encapsulants. The SIRs of the candidates were measured every $6 \mathrm{~h}$ under $100 \mathrm{~V} \mathrm{dc}$. Two test vehicles were coated with Dow Corning Hipec 3-6550 107 


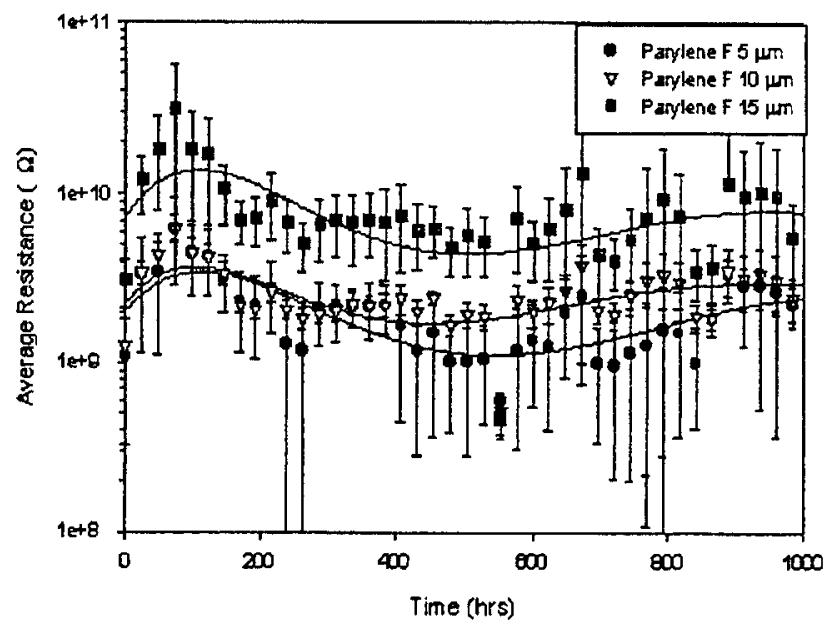

Fig. 5. SIR results for varying film thickness of Parylene F. $\left(85^{\circ} \mathrm{C} / 85 \% \mathrm{RH}\right.$, $100 \mathrm{~V} \mathrm{dc}$ applied on every $6 \mathrm{~h}$ for resistance measurement).

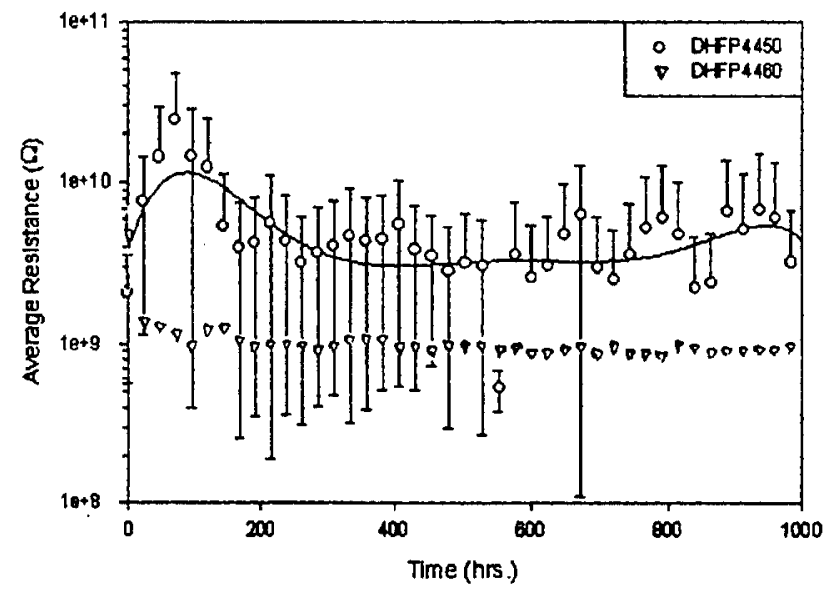

Fig. 6. SIR results for commercial epoxy encapsulants $\left(85^{\circ} \mathrm{C} / 85 \% \mathrm{RH}, 100\right.$ $\mathrm{V} d \mathrm{dc}$ applied on every $6 \mathrm{~h}$ for resistance measurement).

silicone for standardization and to monitor chamber cleanliness. The DC 3-6550 had an initial resistance range of $2.8 \times 10^{9}-4.1$ $\times 10^{9} \Omega$ and completed $1000 \mathrm{~h}$ of testing in the range of 2.7 $\times 10^{9}$ to $3.5 \times 10^{9} \Omega$ (Table I). SIR measurements of the low stress Q1-4939 indicated that the silicone gel of 10:1 provided the best environmental protection (Fig. 3).

Samples coated with $5 \mu \mathrm{m}, 10 \mu \mathrm{m}$, and $15 \mu \mathrm{m}$ films of Parylene $\mathrm{C}$ and $\mathrm{F}$ performed well with resistance value beginning between $1.0 \times 10^{9}$ and $5.0 \times 10^{9} \Omega$ and finishing $1000 \mathrm{~h}$ in the same range (Figs. 4,5 ). Parylene $F$ coatings show a higher degree of environmental protection than their Parylene $C$ counterparts. No visible surface corrosion was observed on the Parylene $F$ coated comb patterns after $1000 \mathrm{~h}$ aging.

SIR measurements of epoxy glob-top encapsulants surveyed indicated that the FP4450 afforded a higher degree of protection than the FP4460 (Fig. 6). The thickness of the epoxy encapsulants was in excess of $76.2 \mu \mathrm{m}$ (equals to 3 mils). The FP4450 showed better environmental protection properties than FP4460 and SIR results of $0.5 \times 10^{9} \Omega$ to $3.0 \times 10^{9} \Omega$ initially and 0.01 $\times 10^{9} \Omega$ to $6.0 \times 10^{9} \Omega$ after $1000 \mathrm{~h}$.

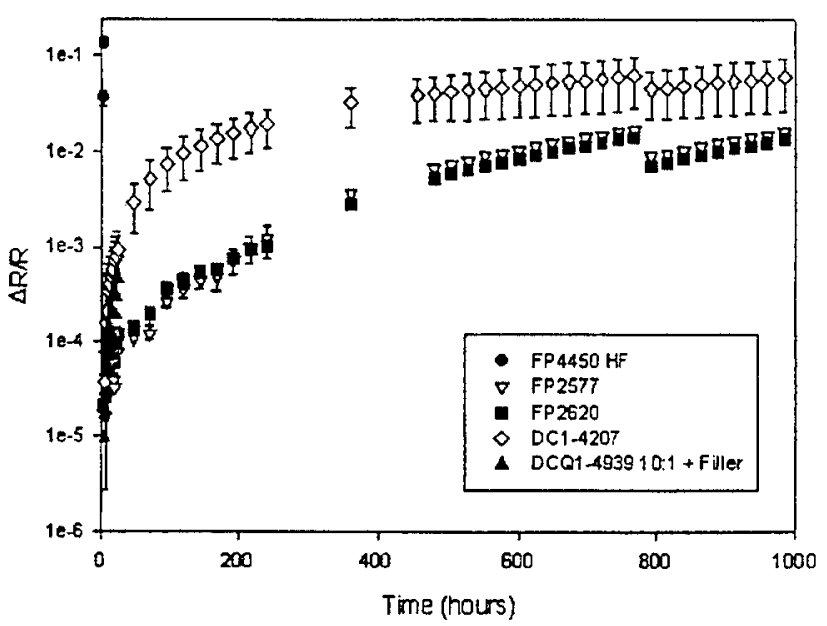

Fig. 7. In-silu triple track resistance measurements for candidate encapsulants. $\left(85^{\circ} \mathrm{C} / 85 \% \mathrm{RH}, 175 \mathrm{~V}^{\prime} \mathrm{dc}\right.$ bias)

TABLE II

Triple Track Resistance Change Data After $1000 \mathrm{~h}$ THB Aging

\begin{tabular}{|c|c|}
\hline \multicolumn{2}{|c|}{ Selected Triple Track Data } \\
\hline Material & Average 1000 Hour $\triangle \mathrm{R} / \mathrm{R}$ \\
\hline Parylene F $15 \mu \mathrm{m}$ & .000166 \\
\hline Parylene C $15 \mu \mathrm{m}$ & .00055 \\
\hline Dow Corning 3-6550 & .0485 \\
\hline Dow Corning $1-4207$ & $.06 !$ \\
\hline Dow Coming QI $4939(10: 1+$ Filler $)$ & Failed \\
\hline Dexter Hysol FP 4450 & Failed \\
\hline Dexter Hysol FP 4651 & Failed \\
\hline Bare Chip & Failed \\
\hline
\end{tabular}

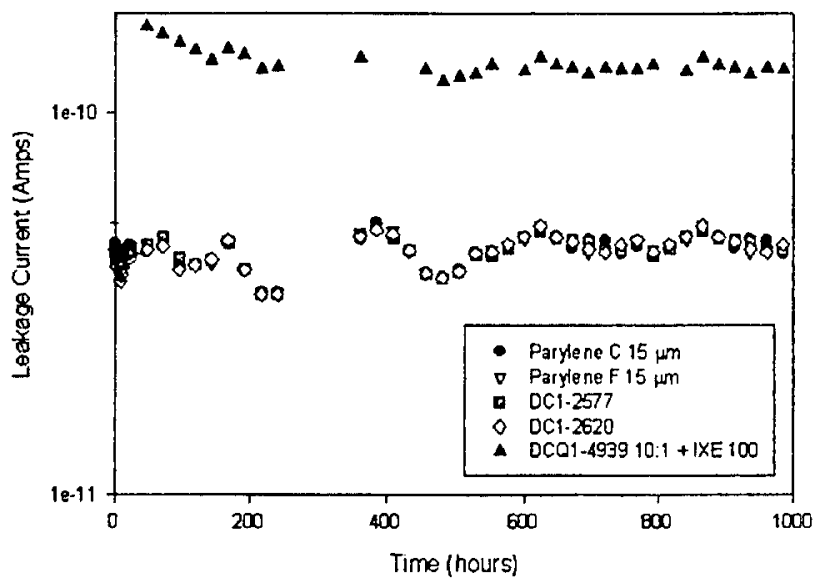

Fig. 8. Triple track leakage current data on the selected conformal coating candidates $\left(85^{\circ} \mathrm{C} / 85 \% \mathrm{RH}, 175 \mathrm{~V}\right.$ dc bias).

In comparison, results from the 3M FC-722 and FC-725 began between $0.0021 \times 10^{9}$ and $0.0056 \times 10^{9} \Omega$. After 1000 $h$, the range had shifted to span from $0.0024 \times 10^{9}$ to $0.0029 \times$ $10^{9} \Omega$ for the FC-722 and from $0.0071 \times 10^{9}$ and $0.0058 \times 10^{9}$ $\Omega$ for the FC 725 . These results indicate an inferior resistance to moisture ingress. So these two materials were discounted from further qualification testing (Table I). 


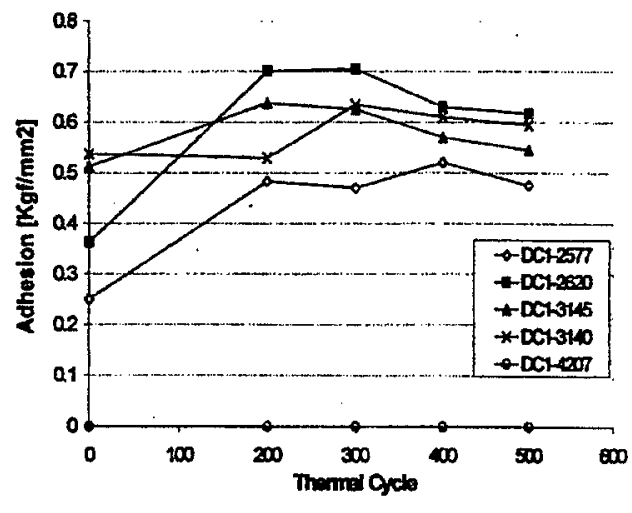

(a)

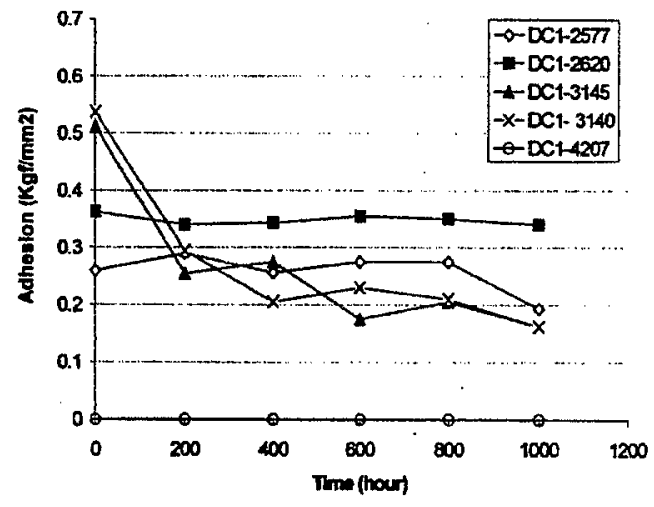

(b)

Fig. 9. Adhesion changed with aging time for monolayer silicone elastomers on polyimid passiviated Si substrates: (a) thermal shock $\left(-55\right.$ to $\left.125^{\circ} \mathrm{C}\right)$ cycling and (b) temperature/humidity $\left(85^{\circ} \mathrm{C} / 85 \% \mathrm{RH}\right)$ aging.

\section{B. Triple Track Test (TTT) Measurements}

TTT was employed to screen potential candidates in terms of their environmental protection property. For qualification screening of potential candidate materials, the TTT vehicles as shown in Fig. 3(a) was selected. Resistance measurements were recorded along each metal track in the serpentine pattern. The change in resistance would prove to be the primary factor in determining the failure of test vehicles during triple track measurements. A chip failure was defined as any drastic change in the resistance of the triple track test vehicle. In general, resistance values were seen to slowly increase over time due to corrosion of the $\mathrm{Ta}_{2} \mathrm{~N}$ metallization pattern. When a vehicle failed, the slope of the change-in-resistance plot would rapidly increase. Once initiated, the failure process occurred in less than $24 \mathrm{~h}$. Chips that did not fail within the initial $24 \mathrm{~h}$ could still be examined for relative merit. The epoxy glob-top encapsulants revealed their inherent weakness in a high humidity environment, resulting from the presence of epichlorohydrin in the base agent that increased the presence of chloride ion contaminants. Epoxies typically failed quickly, like the FP4450 HF shown in Fig. 7, which failed within $48 \mathrm{~h}$. Silicone elastomers all showed similar trends, but the DC1-4207 was clearly inferior to DC1-2620 or DC1-2577. The shift seen in the plot around $750 \mathrm{~h}$ is due to system irregularities. In the case of Parylenes, $15 \mu \mathrm{m}$ coatings showed similar excellent moisture resistance protection results, as indicated in Table II.

The leakage current values do not provide convenient answers as trip track resistance. The noise floor of the testing apparatus was found to be roughly $4.0 \times 10^{-11} \mathrm{~A}$ as established by the silicone elastomers and Parylenes in Fig. 8. The data does indicate that the silicones and Parylenes show excellent resistance to moisture and mobile ion permeation. The actual leakage current values were $<4.0 \times 10^{-11} \mathrm{~A}$, indicating that the triple track test chips were still protected. Chips that had suffered mobile ion permeation would experience leakage current values that would rise above the noise floor. This would differentiate the good and bad conformal coatings. Samples such as Hipec Q1-4939 10:1 mixed with some filler generated bad results.

\section{Long Term Hostile Environmental Conditioning for Die Shear Testing}

Preparation of single-layer and bi-layer coating samples had been done for evaluating the long-term adhesion reliability. The single-layer and bi-layer coated test vehicles were subjected to thermal shock cycling and temperature humidity aging with 100 cycles and $200 \mathrm{~h}$ intervals, respectively.

Samples subjected to these two testing protocol consisted of five single-layer, 15 bi-layer combination coatings. The singlelayer samples include the following silicone elastomers: DC12577, DC1-2620, DC1-4207, DC1-3140, and DC1-3145. All silicone samples were coated on polyimide passivated Si substrates. The 10 bi-layer combination coatings included the above silicone elastomers coated with Parylene $\mathrm{C}$ using two novel adhesion promoters to serve as adhesion promoters. The additional five bi-layer coatings were combinations of FP4460 and the above five silicones. FP4460 was selected as a base layer due to the known reliable property for the ASICS glob-top protection. Bi-layer combinations were selected to mimic the nonhermetic coating approach for the MEMS device. Results from the thermal shock cycling and temperature humidity aging testing indicate that the DC 1-2620 has the strongest adhesion for a single-layer design. For bi-layer designs, DC1-3145 silicone in combination with Parylene $\mathrm{C}$ showed the highest adhesive strength. Alternatively, DC 1-2620 with FP 4460 epoxy showed the best strength in comparison to other silicone candidates used with epoxy. Results from these investigations are outlined in Figs. 9-12.

Fig. 9 shows that the DC1-2620 conducts the best adhesion between silicones and polyimide passiviated Si substrates. Adhesion between silicone candidates and PPS substrates initially increase after exposure to thermal shock cycling, however, after a certain stable period, the samples started to decrease due to the CTE mismatch. While adhesion variation after exposure to $85^{\circ} \mathrm{C} / 85 \% \mathrm{RH}$ shows continuous decreasing with the aging time, which might be caused by the diffusion or release of the oligmers escaped from silicone bulky matrix.

Adhesion for bi-layer of silicone elastomers and FP4460 changed with aging time is displayed in Fig. 10. The adhesion 109 


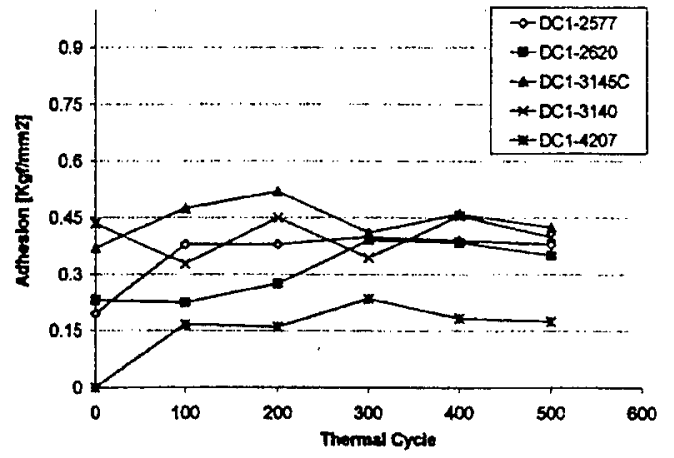

(a)

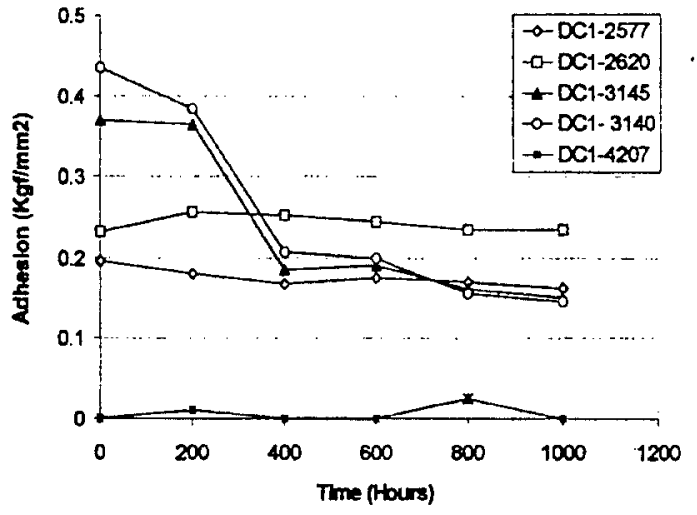

(b)

Fig. 10. Adhesion between silicone elastomer and FP4460 epoxy changed with aging time for bilayer conformal coating system: (a) thermal shock (-55 to $\left.125^{\circ} \mathrm{C}\right)$ cycling and (b) temperature/humidity $\left(85^{\circ} \mathrm{C} / 85 \% \mathrm{RH}\right)$ aging.

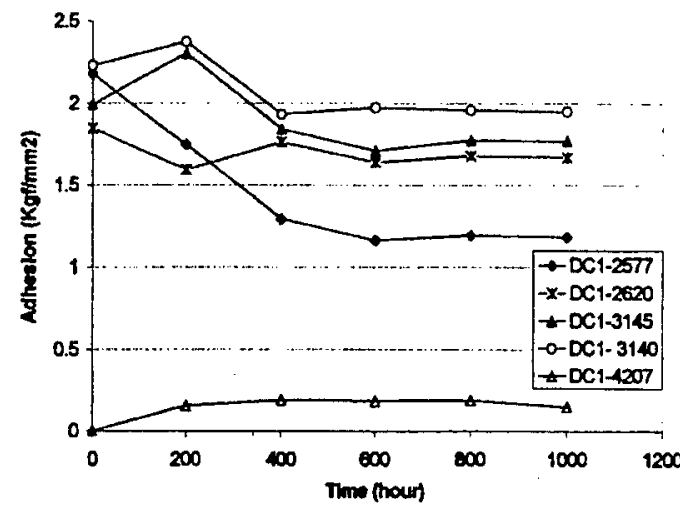

(a)

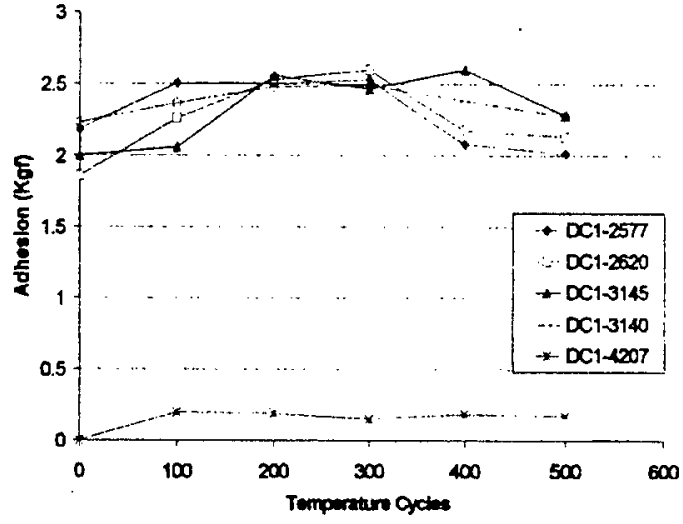

(b)

Fig. 11. Adhesion between silicone elastomer and Parylene $\mathrm{C}$ changed with aging time for bi-layer system with Adhesion promoter AP-B: (a) temperature/humidity $\left(85^{\circ} \mathrm{C} / 85 \% \mathrm{RH}\right)$ aging and (b) thermal shock $\left(-55\right.$ to $\left.125^{\circ} \mathrm{C}\right)$ cycling.

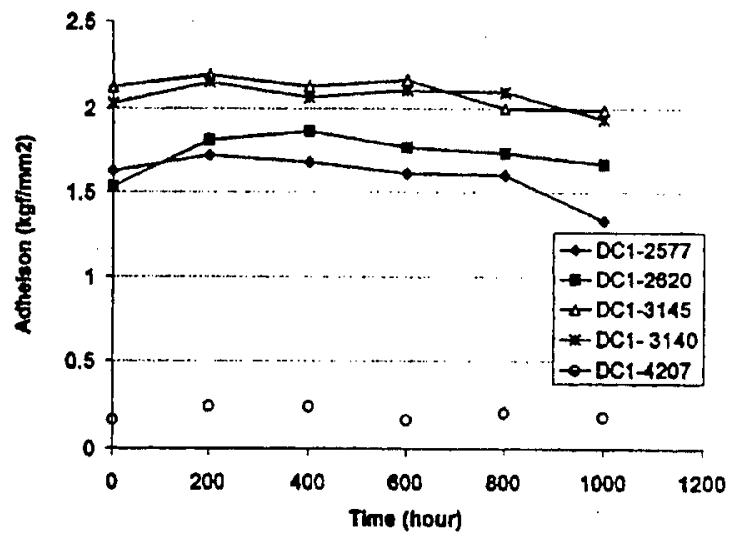

(a)

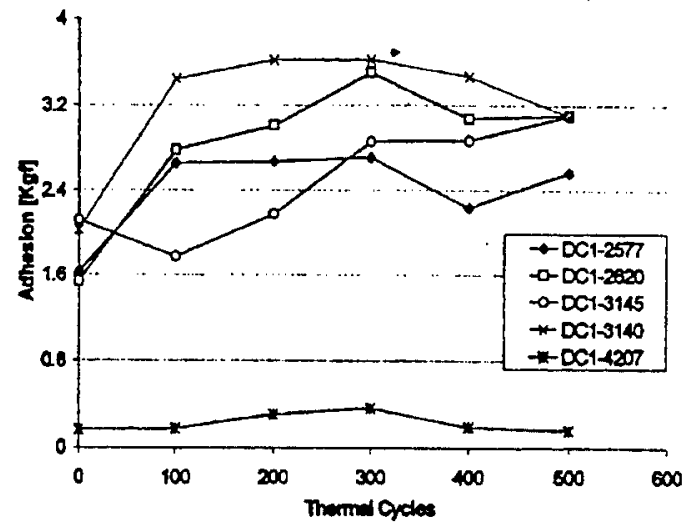

(b)

Fig. 12. Adhesion between silicone elastomer and Parylene $\mathrm{C}$ changed with aging time for bi-layer system with Adhesion promoter AP-A; (a) Temperature/Humidity $\left(85^{\circ} \mathrm{C} / 85 \% \mathrm{RH}\right)$ aging; (b) Thermal Shock $\left(-55\right.$ to $\left.125^{\circ} \mathrm{C}\right)$ cycling

continuously decreases when the samples were exposed to $85^{\circ} \mathrm{C} / 85 \% \mathrm{RH}$ atmosphere. While the adhesion show a slight change for samples during the course of thermal shock cycling testing, but not as much as that of single-layer silicone on the polyimide passiviated Si substrate.
Adhesion variations for bi-layer of silicone elastomer and Parylene $\mathrm{C}$ after $85^{\circ} \mathrm{C} / 85 \% \mathrm{RH}$ aging and thermal shock cycling are illustrated in Figs. 11,12 . After the $85^{\circ} \mathrm{C} / 85 \% \mathrm{RH}$ aging, adhesion for both of AP-A and AP-B bi-layer samples tended to decrease continuously. While samples exposed to thermal shock 110 
cycling remained their adhesion. This result indicates that the AP-A is a more effective adhesion promoter to the interfacial adhesion.

\section{CONCLUSION}

In this investigation, preliminary results have been outlined for candidate coatings that show promise as effective nonhermetic encapsulants for MEMS device applications. Comparison of SIR and TTT results indicate that the silicone elastomers and Parylenes type $\mathrm{F}$ and $\mathrm{C}$ display excellent resistance to moisture ingress and mobile ion permeation and are viable moisture and mobile ion permeation barrier candidates. The 10:1 ratio (part A: part B) of Q1-4939 is an excellent candidate for applications requiring a low stress encapsulant that also provides good environmental protection. The epoxy based FP4450 and FP4460 epoxy encapsulants exhibit excellent adhesion and fair resistance to moisture ingress, which make them become attractive glob-top candidates. Silicone elastomers show significant promise as a planarizing top-layer that will afford additional environmental protection for enhanced high-reliability applications. Results indicate that the fluoroacrylic coatings evaluated in this study were inappropriate for encapsulation of MEMS devices. Results from additional in-depth investigations including military specification environmental protection qualification testing is of significant interest and will be further examined in this program.

\section{ACKNOWLEDGMENT}

The authors wish to thank Dr. M. Tanielian, Dr. N. Kim, and M. Chisa, Boeing Company, for funding this project and providing test vehicles and materials, W. Yager and J. Yira, Specialty Coating Systems, Inc., for Parylene F and C test vehicle encapsulation, A. Smith, Georgia Tech, for assistance with the triple track software programming, and Dr. A. Janata, Georgia Tech, for supplies.

\section{REFERENCES}

[1] A. W. Lin and C. P. Wong, "Encapsulant for nonhermetic multichip packaging applications," IEEE Trans. Comp., Hybrids, Manufact. Teclinol., vol. 15, pp. 510-518, Aug 1992.

[2] D. Jaffe, "Encapsulation of integrated circuits containing beam lead devices with a silicone RTV dispersion," IEEE Trans. Parts, Hybrids Packag., vol. 12, p. 182, 1976

[3] R. A. Mancke, "A moisture protection screening test for hybrid circuit encapsulants," IEEE Trans. Comp., Hybrids, Manufact. Technol., vol. 4, p. $492,1981$.

[4] C. P. Wong, "Application of polymer in encapsulation of electronic parts," in Advances in Polymer Science. Berlin-Heidelburg, Germany: Springler-Verlag, 1988, vol. 48, ch. 2, pp. 63-83.

[5] - "Integrated Circuit Device Encapsulants," in Polymer for Electronic Applications. Boca Raton, FL: CRC, 1989, ch. 3, pp. 63-92.

[6] C. P. Wong, J. M. Segelken, and C. N. Robinson, "Chip on Board Encapsulation," in Recent Advances in IC Passivation and Encapsulation: Process Techniques and Materials. San Diego, CA: Academic, 1992 ch. 11, pp. 471-501.

[7] C. P. Wong, "Effects of RTV silicone cure in device packaging," in Proc. Polymer Sci. Eng., vol. 55, 1986, p. 803.
[8] —-, "IC encapsulants," in Polymer Materials for Electronic Applica tions. Berkeley, CA: Univ. California Press, August 1993, ch. 6, p. 12.

[9] N. Sinnardurai," "An evaluation of plastic coatings for high reliability microcircuits," Microelectron. J., vol. 12, no. 6, 1981

[10] C. P. Wong and D. M. Rose, "Alcohol modified RTV silicone encapsulants for IC device packaging," IEEE Trans. Comp., Hybrids, Manufact. Technol., vol. CHMT-6, 1983.

[11] C. P. Wong and R. McBride, "Preencapsulation cleaning methods and control for microelectronics packaging," IEEE Trans. Comp., Packag., Manufact. Technol. A, vol. 17, p. 542, Dec. 1994.

[12] J. Wu, T. Pike, and C. P. Wong, "Novel bi-layer conformal coating for reliability without hermeticity MEMS encapsulation," IEEE Trans. Comp., Packag., Manufart. Technol. C, vol. 22, p. 195, July 1999.

Jiali Wu (M'99) received the B.S. degree in chemistry from Zhejiang University, China, and the M.S. and Ph.D. degrees in chemistry from the Shanghai Institute of Metallurgy, Chinese Academy of Sciences, Shanghai, China.

From 1991 to 1997, her major research work focused on microsensor fabrication and application in electrochemistry field and die bonding with Au/ln bi-alloy isothermal solidification technique. She has been with the Electronics Packaging Research Group, School of Materials Science and Engineering, Georgia Institute of Technology, Atlanta, as a Postdoctoral Fellow since 1997. She is currently working on polymer development for the application of various electronics packaging, mainly including multilayer conformal coatings for reliability without hermeticity encapsulation, reworkable high temperature adhesives for advanced MCM-D processing, underfills, and parylene interfacial adhesion enhancement on polymeric passiviated substrates.

Randy T. Pike, photograph and biography not available at the time of publication.

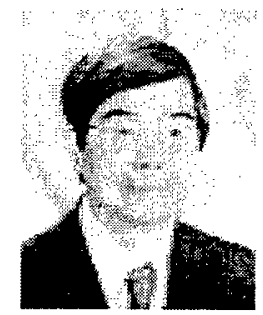

C. P. Wong (SM'87-F'92) received the B.S. degree in chemistry from Purdue University, West Lafayette, IN, and the Ph.D. degree in organic/inorganic chemistry from Pennsylvania State University, University Park.

He was a Postdoc Fellow at Stanford University Stanford, CA. He spent 19 years with AT\&T Bell Labs and was elected a Bell Labs Fellow in 1992. $\mathrm{He}$ is a Regents' Professor with the School of Materials Science and Engineering and a Research Director with the Packaging Research Center, Georgia Institute of Technology, Atlanta. He holds over 45 U.S. patents, numerous international patents, and over 240 technical papers in the related area. His research interests lie in the fields of polymerica materials, reaction mechanism, IC encapsulation, hermetic equivalent plastic packaging, electronic packaging processes, interfacial adhesions, PWB, SMT assembly, and component reliability.

Dr. Wong received the AT\&T Bell Laboratories Distinguished Technical Staff Award in 1987, the AT\&T Bell Labs Fellow Award in 1992, the IEEE Components Packaging and Manufacturing Technology (CPMT) Society Outstanding and Best Paper Awards in 1990, 1991, 1994, 1996, and 1998, the IEEE Technical Activities Board Distinguished Award in 1994, the 1995 IEEE CPMT Society's Outstanding Sustained Technical Contribution Award, the 1999 Georgia Tech's Outstanding Faculty of the Year Award, the Georgia Tech Sigma Xi Faculty Best Research Paper Award in 2000, and the University Press (London, U.K.) Award of Excellence in 2000. He is a member of the National Academy of Engineering, a Fellow of AT\&T Bell Labs, and was the General Chairman of the 41st Electronic Components and Technology Conference in 1991. He served as Technical Vice President (1990 and 1991) and President (1992 and 1993) of the IEEE CPMT Society.

111 


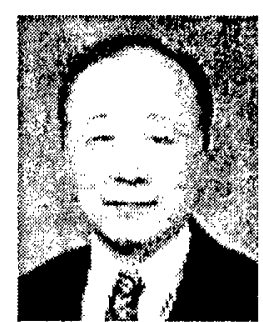

Namsoo P. Kim received the B.S. degree from Seoul National University, Seoul, Korea, in 1972, and the M.S. and Ph.D. degrees in material science and engineering from the University of California, Los Angeles, in 1979 and 1981, respectively.

After graduation, he worked at American Microsystems, Inc. (AMI), Santa Clara, CA, as Manager of the Packaging and Materials Department. His research involved material issues for silicon integrated circuit fabrication and packaging technologues. In 1986, he joined Boeing where he is Associate Technical Fellow in the Department of Information, Communication. Sensor \& Electronics Technologies, Boeing Phantom Works, Seattle, WA. He is Technical Principal Investigator for various packaging research programs utilizing emerging component technologies including micro-electro-mechanical system (MEMS), CSPs, and flip chips. His past research programs included application of thin and thick film materials for multichip modules and photovoltaic devices. He is an author or co-author of over 40 journal and conference papers and three U.S. and international patents.

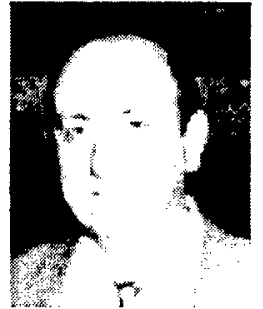

Minas H. Tanielian received the B.S., M.S., and $\mathrm{Ph} . \mathrm{D}$. degrees from the University of Chicago, Chicago, IL, in 1974, 1975, and 1981, respectively, all in solid state physics.

He is the Manager of the Sensor and Microsystems Technology Organization, Boeing Phantom Works, Seattle, WA. His interests span a variety of technologies and applied physics topics such as thin film materials, electronic devices, photonics, and microsystem integration. Current activities relate microsystem applications. 


\title{
Smart Sensor Multi-Chip Module With Direct-Chip-Attached Micro-Electro-Mechanical System (MEMS) Device
}

\author{
Namsoo P. Kim, Kin Li, Daniel J. Kovach, Chung-Ping Chien, and Minas H. Tanielian \\ Boeing Phantom Works \\ Information, Communication, Sensor \& Electronics Technologies \\ P.O. Box 3999, MS 3W-50 \\ Seattle, WA 98124-2499 \\ (253) 657-5970, e-mail: namsoo.p.kim@boeing.com
}

\begin{abstract}
Single Micro-Electro-Mechanical System (MEMS)-type sensors have been used for a variety of automotive and medical applications. However, there has been very little activity in the area of nultisensor systems. Boeing is developing a MEMS multisensor system suitable for aerospace applications, including the hardware, firmware, and software necessary to run such a system. In this paper, we report the development of a smart sensor multi-chip module (MCM) containing MEMS-based pressure transducers. The module contains a bare MEMS device, and a CMOS Analog Signal Processor ASIC, an EEPROM and a number of passives. The MCM-D substrate was fabricated on oxidized silicon using copper as conductor and photo-sensitive polyimide as dielectric material. Assembly of the module was conducted by utilizing various microelectronic interconnection techniques. The MEMS device was bonded using direct-chip-attachment technique (flip chip) to minimize the height. Finally silicone molding was applied to the module for environmental protection. Functionality was tested by measuring electrical responses as a function of pressure and temperature.
\end{abstract}

Keywords: Micromechanical Systems (MEMS), pressure sensor, direct chip attachment (DCA), flip chip, MCM

\section{Introduction}

Boeing utilizes many different sensor systems for its aircraft and aerospace systems. During load surveys of commercial and military aircraft, arrays of many sensors are required on a common bus. Microelectromechanical system (MEMS) based sensors, in spite of their popularity in single sensor configurations, have not been widely implemented in array configuration due to lack of packaging and communication infrastructure. Boeing has been developing multiMEMS sensor systems to take full advantage of this important technology. Electronic circuitry at the point-of-use is needed to provide robust communication with the host, and for temperature compensation and self-calibration. A thin and/or miniaturized profile is also needed for aerodynamic requirements.

In this paper, we report development of a smart sensor multi-chip module (MCM) containing MEMSbased pressure transducers suitable for multi sensor applications. The prototype modules were built, and tested for full functionality. These modules were incorporated into the common bus and installed onto the aircraft outside surface producing valuable flight test sensor data.

\section{System Requirements and Module Description}

Two major parameters driving our system and module development were the thin profile and sensor accuracy. Other parameters taken into consideration were structural compatibility with target vehicle, operational temperature range, resistance to moisture and fuel vapor, and other general reliability constraints. The target accuracy of $0.1 \%$ of full scale is about an order better than the typical accuracy possible with the current practices using tubing belt pressure measurement. To meet this objective, module configuration and all the material systems were carefully chosen to minimize any stress being passed onto the sensor diaphragm. Stress can be transmitted from any underlying structure such as the airplane wing or even the MCM substrate itself. It was one of the main reasons why we decided to incorporate MCM substrates with size smaller than 1 in. square so that it is mountable on curved surfaces. Thin profile of the module (approximately $0.070 \mathrm{in}$.) was needed for aerodynamic reasons.

The prototype modules built and tested for this program utilized a bare MEMS device and a commercially available CMOS analog signal conditioner (MCA 7707) providing a ratiometric output. Calibration and compensation were 
controlled by on-module memory (EEPROM.) Its mechanical packaging configuration was proven through flight testing and will remain the same for the future version module utilizing two Boeing designed ASIC components. Its main electrical functionality addition will be local digitization with a transducerto-bus interface. It will also contain a higher order correction engine.

\section{MEMS PRESSURE SENSOR}

The MEMS sensor that we used for this program is a modified version of the standard product offered by a commercial manufacturer. It is a ion-implanted silicon micromachined pressure sensor whose diaphragm is designed for a $0-15$ psia pressure range. It incorporates a fully active Wheatstone bridge strain sensing circuit on the internal surface of the diaphragm. This rectangular sensor with .049 "x.067" dimensions is shown in Figure 1 (a) and (b). This is an absolute reference sensor. The vacuum reference is accomplished with a second silicon support attached to the back of the diaphragm. The support is hermetically glass sealed to the sensor and has a cavity which is evacuated during the sealing process as schematically shown in Figure 2.

While the diaphragm currently in use is a standard part, the reference support has been specifically designed to allow flip chip mounting of the MEMS assembly to the silicon MCM using solder bumps or conductive epoxy. This maintains the lowest possible profile since no wires extend above the top of the MEMS device which is only .016 inch thick. The special flip chip support provides feedthroughs so that electrical connections can be made from the backside of the sensor assembly. The feedthroughs consist of etched holes through the reference support. Pad metallization is then deposited which creates electrical bonding sites on the surface of the support and connects them to the pads on the surface of the diaphragm at the bottoms of the feedthroughs.

\section{Substrate Design}

The 0.8 " $x 0.8$ " substrate was designed for the MCM using copper as conductor and polyimide as dielectric. It was laid out using Tanner LEDIT software using the parts library and the fabrication and packaging design guide lines. Three MEMS sensor sites were allocated to assess the effect of location in the module on the sensor sensitivity. Even though only the one in the center has associated electronic circuitry, high local wiring density became needed since no wiring is allowed underneath MEMS components. The width and pitch of the conductor lines was $1 \mathrm{mil}$. Figure 3 shows the cross section of the prototype module. Even though we can add more conductor and dielectric layers easily due to modular nature of our process, we incorporated the most simple structure using two metal conductor and a single dielectric layers since it offered higher yield, higher reliability and lower cost. Boeing's 7 layer MCM was demonstrated early and described in details [1.]

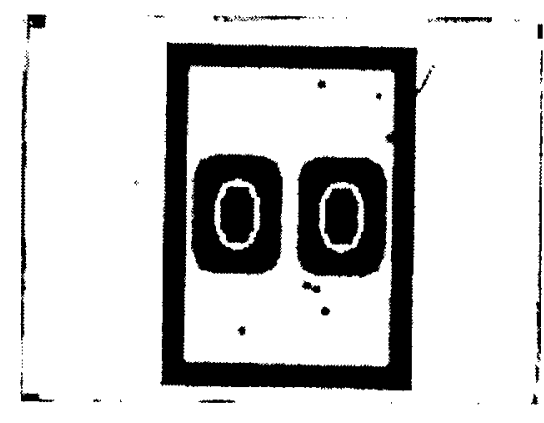

(a) Front side

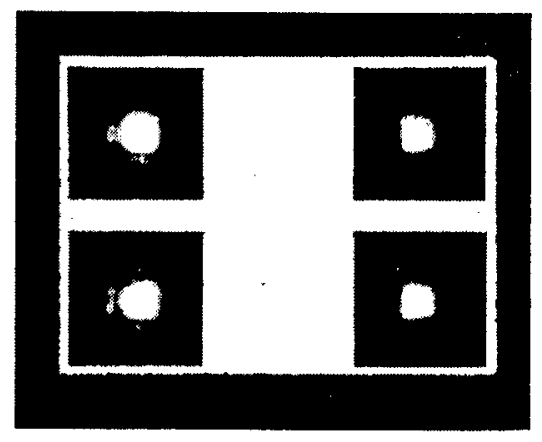

(b) Back side

Figure 1. Photograph of redesigned pressure sensor

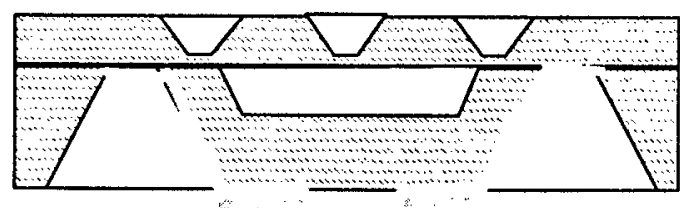

Figure 2. MEMS device schematic

This MEMS module also contained the additional metal films suitable for MEMS attachment and bond pads. A unique design trade-off was with the attachment of MEMS flip chip bonding. Direct bonding to silicon oxide coated surface rather without polyimide layer was needed to eliminate any residual stress coming from cured polyimide and also from thermal coefficient mismatching. This opening through polyimide layer also contained the site for 


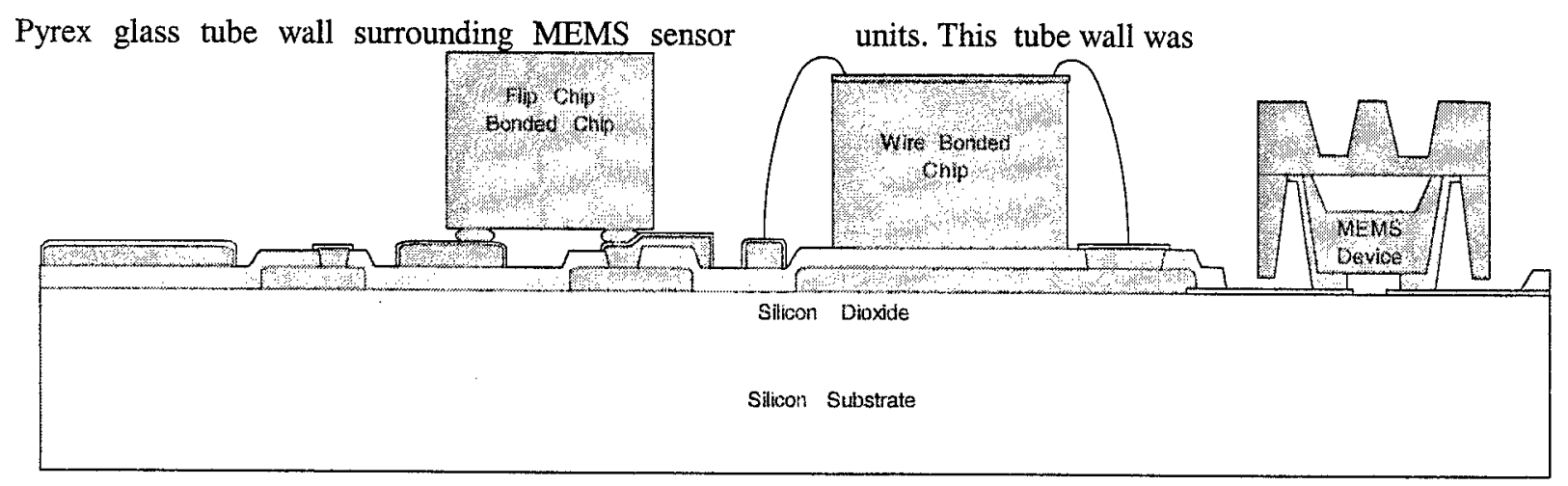

Figure 3. MCM cross section schematic

needed not only to provide more aerodynamically acceptable surface profile for sensor accuracy and also to prevent any handling damage to MEMS sensor during the assembly and installation. The module site contained seventeen passive components in addition to the MEMS and two ICs described above. Due to the relatively thick copper line used in this process, line resistance between any two point in our MCM substrate did not exceed 2 ohms. A 5" silicon wafer contained $12 \mathrm{MCM}$ units.

\section{Substrate Fabrication}

The MCM substrate was fabricated on a $10000 \AA$ oxidized silicon using 6 mask steps. The first metallization layer was a Titanium/Nickel/Gold (500/1500/2500 A) stackup deposited by electronbeam evaporation. This layer served as site for direct chip attachment of the MEMS device offering compatibility with solder and epoxy based flip chip. Both conventional patterning with subtractive etch techniques and lift-off processes were used successfully for this step, but the latter offered more simplicity. A Titanium/Copper $(500 \AA / 3 \mu \mathrm{m})$ conductor layer was sputtered onto the wafer, then the copper surface was patterned and wet etched. Another $500 \AA$ layer of Titanium was sputtered, then the Titanium was patterned and (both layers of $\mathrm{Ti}$ ) dry etched in an RIE.

The dielectric employed in this MCM was a preimidized photo-sensitive polyimide, Amoco's Ultradel 7501. After depositing an adhesion promoter $(\gamma-\mathrm{APS})$, the polyimide was spin coated and softbaked. A combined 3 minute hotplate bake @ $100 \mathrm{C}$ with an 1 hour N2 oven bake @ 160C was employed with good results. The polyimide was patterned in a projection aligner using broadband UV, then spray developed using $\gamma$-butyrolactone/isopropanol as developer/rinse.
Following a plasma descum, the layer was given an additional UV flood-expose followed by a thermal cure in a vacuum oven. The second metal conductor stack was added in the same way for the first layer. An alternative process for this step was electroplating of copper after sputtering a Ti/Cu $(500 \AA / 5000 \AA)$ seed layer. Both process produced good results while the latter offered the layer with less stress. Its processing details are described in another paper at this conference [2.] Following this, the final pad layer $(\mathrm{Ti} / \mathrm{Ni} / \mathrm{Au})$ was deposited and patterned. $\mathrm{A}$ layer of copper was then deposited, patterned and etched as hard mask to dry etch through the dielectric layer, exposing the MEMS pads which had been deposited in the beginning of the process. Removing the hard mask and cleaning completed the fabrication process. Figure 4 shows a completed MCM wafer containing $12 \mathrm{MCMs}$ and figure 5 shows one unpopulated MCM unit.

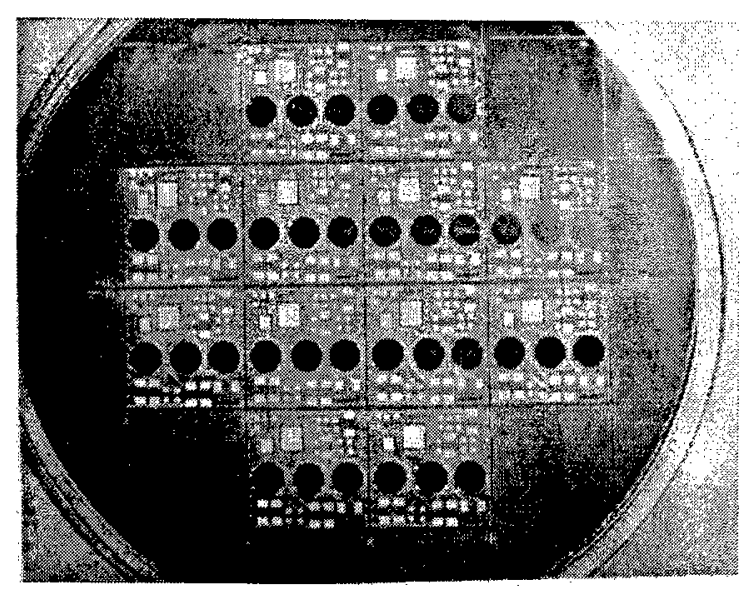

Figure 4. Completed functional MCM wafer.

Completed wafers were tested electrically for open and shorts. They were also subjected for burn-in 
screening test to eliminate any infant mortality. This consisted of three cycles ranging from room temperature to $250{ }^{\circ} \mathrm{C}$ and seven additional cycles ranging from -55 to $125^{\circ} \mathrm{C}$. These wafers were then diced to produce good MCM units to be used for module assembly.

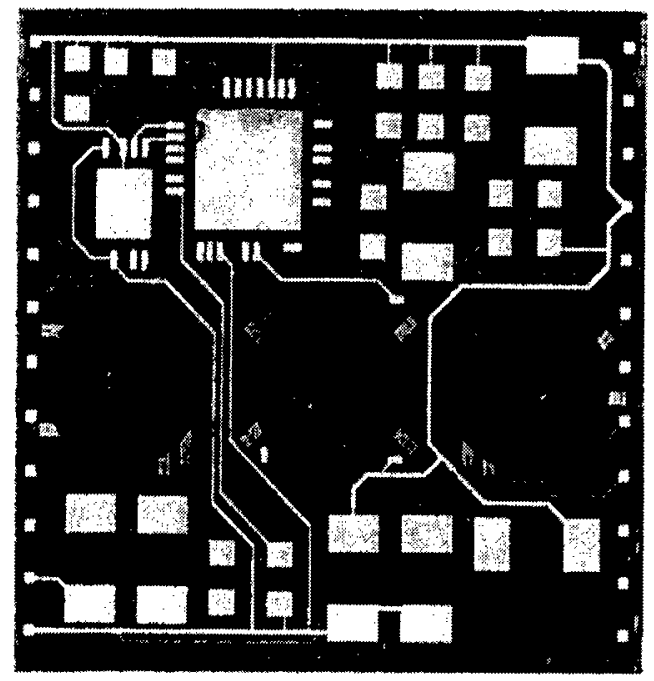

Figure 5. An MCM substrate containing 3 MEMS sites. It is 0.8 by 0.8 inches in size.

\section{Module assembly}

Module assembly was conducted by attaching various components to the MCM substrate. Various microelectronic assembly process were employed. Choice of these processes was somewhat limited since commercially available components were incorporated for this version module. MEMS devices and discrete components were attached to the MCM substrate using Ablestik conductive epoxy while the MCA7707, the EEPROM ICs and the tube wall were bonded using nonconductive epoxy. Gold wedge bonding was used for the MCA and EEPROM ICs electrical connection since they were not available in flip chip compatible wafer form. Figure 6 shows a fully populated MCM unit used, while Figure 7 shows close-up detail. These fully populated MCM units were functionally tested using the test probe board and the test procedures developed for process monitoring. The functional test consists of applying power, establishing serial data communication, and verifying valid signal response from the MCA7707 circuitry. Vacuum was applied to the MEMS sensors and repeatable signal levels were observed within an acceptable range of the anticipated outputs.

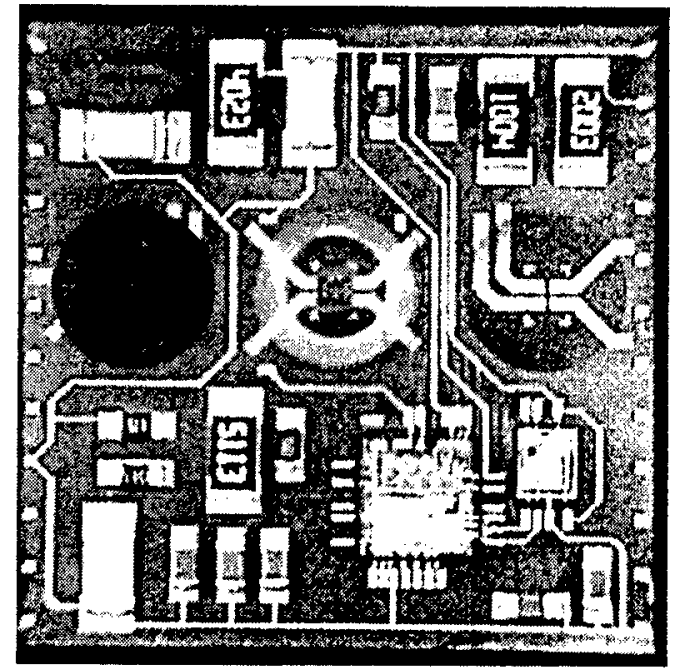

Figure 6. A fully populated MCM. Unit

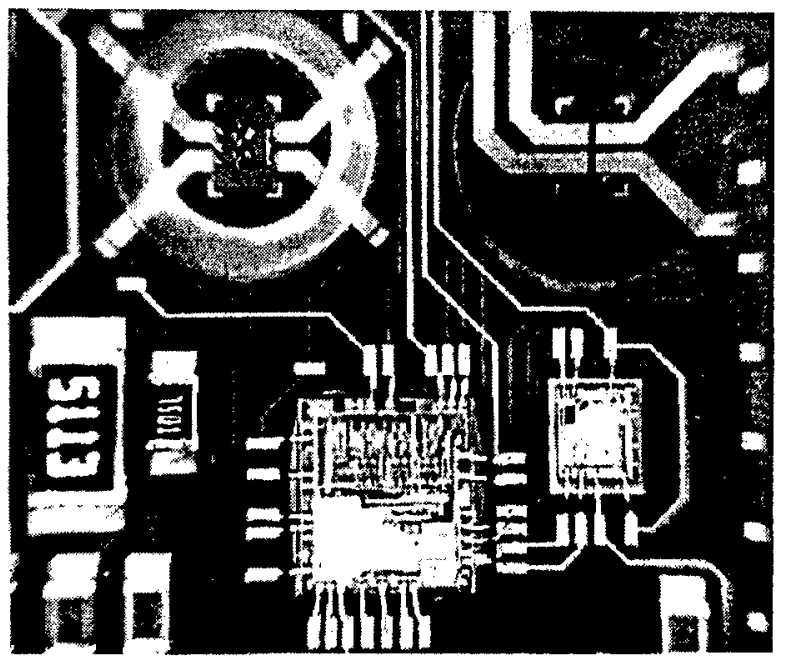

Figure 7. Close-up detail of MEMS and ICs.

Dow Corning DC Q1-4939 (a very low stress gel) was applied between the MEMS device and the tube wall to protect the base region of the MEMS device and to prevent water invasion. Hysol Dexter 4460 (glob-top) epoxy was applied to protect both ICs and another functional test was conducted completing module assembly.

\section{Results and Discussion}

Functionally tested module units were mounted to Tape Automated Bonding (TAB) carrier using 3M $468 \mathrm{MP}$ adhesive to form a pressure belt segment. 
This adhesive was chosen for optimum characteristics of robust adhesive strength and minimum stress transmission. The TAB carrier was polyimide film in ANSI $70 \mathrm{~mm}$ format and contained $\mathrm{Cu}$ conductor wiring. Silicone coating of Dow Corning DC1-2577 was applied to protect MCM components and beam leads after interconnection of the MCM to the carrier. Figure 8 shows a portion of pressure belt segment depicting one coated MCM unit. The pressure belt containing 6 units was installed on Boeing 757 with fairing butted to the sensor tube wall. This flight test indicated very favorable results producing $0.05 \%$ full scale deviations from the reference pressure tube measurements, well below our target value. Data averaging incorporated was using 30 data points collected at the cruising altitude of 15,000 feet. Time elapsed for each data point was 6 seconds ( 5 samples per second data acquisition.) Details of the flight test results and the structure of the full pressure belt are beyond the scope of this paper and will be published at a later date. Some units were retrieved from this test and used for another flight test also producing the well behaving second flight test pressure data.

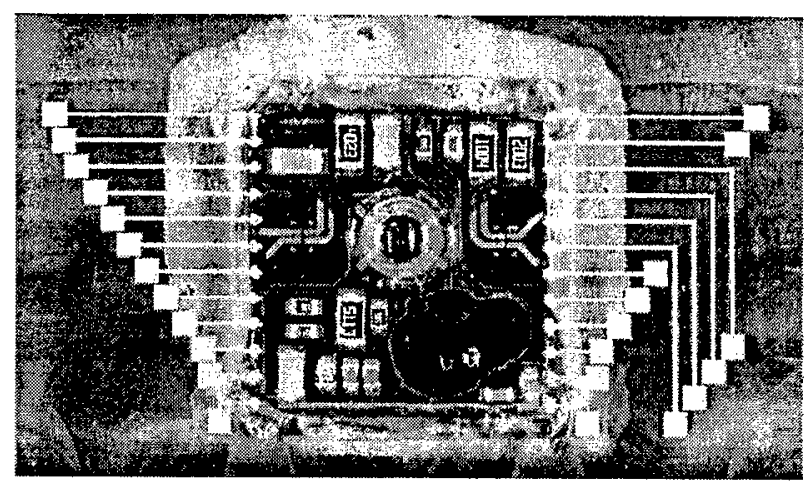

Figure 8. Assembled Segment portion of pressure belt

A new generation of the pressure belt is being designed with additional improvements. Incorporation of two Boeing designed ASICs with flip chip compatible pads will make the new generation module even lower profile with added electrical functionality for local digitization, robust communication and higher order correction engine. This MCM will also utilize embedded passives elements eliminating discrete components. Development of those embedded passives were described elsewhere $[3,4$.

\section{Conclusion}

Development of a smart sensor module suitable for multi-sensor system applications is reported. Its mechanical configuration was confirmed through two actual flight tests. When implemented, significant cost savings in our aircraft and aerospace systems are expected because not only it is lower cost, but it also helps to make calibration and installation easier and more simplified.

\section{Acknowledgments}

This work was supported by DARPA/AF Research Lab under the agreement F30602-97-2-0099. The authors would like to thank MEMS Pressure Belt IPT members, especially M. Holland for his flight test support. Thanks are also extended to $M$. Chisa and $\mathrm{H}$. Soares for module assembly, and J. Nielsen L. Branson, C. Littlejohn, K. Coates, and N. Amirgulyan for substrate fabrication. We also acknowledge Endevco's support, especially by R.Poff, J. Fragala, B. Wilner, and A. Karolys for device supply.

\section{References}

1. J. Cech et al, "Reliability of Passivated Copper Multichip Module Structures Embedded in Polyimide," IEEE Trans. CHMT, v16, No 7, p.752, 1993

2. D. Kovach et al., "A Nearly Stress-Free Cu/Polyimide Process For Large Format MCM Manufacturing," this conference

3. K. Coates et al, "Development of Thin Film Resistors for Use in Multichip Modules," Inter. Conf. On Multichip Modules and High Density Packaging, p. 490, 1998

4. N.P. Kim, K.L. Coates, G.G. Kunze, C-P. Chien, and M.H. Tanielian, "Development of MultiChip Modules with Integrated Thin Film Passive Elements," Proc. Of $30^{\text {th }}$ International Symp. Of Microelectronics (IMAPS 97), p. 157, 1997 


\title{
MEMS Sensor Multi-chip Module Assembly with TAB Carrier - Pressure Belt for Aircraft Flight Testing
}

\author{
Namsoo P. Kim, Mark J. Holland ${ }^{1}$, Minas H. Tanielian, and Ron Poff ${ }^{2}$ \\ Boeing Phantom Works \\ Information, Communication, Sensor \& Electronics Technologies \\ P.O. Box 3999, MS 3W-50, Seattle, WA 98124-2499 \\ (253) 657-5970, e-mail: namsoo.p.kim@boeing.com \\ ${ }^{1}$ Boeing Commercial Airplane Group, Flight Test, Seattle, WA \\ ${ }^{2}$ Endevco Corp., San Juan Capistrano , CA
}

\begin{abstract}
Many different sensor types and their associated electronic systems are utilized for aircraft and aerospace applications. When atmospheric data is collected like pressure load surveys for aircraft, arrays of many sensors are required on a common bus. With the support from a device manufacturer, we have been developing a multi-Micro-Electro-Mechanical System (MEMS) sensor based electronic system. We report in this paper a unique electronic packaging configuration incorporating MEMS sensors, multi-chip modules (MCMs) and tape automated bonding (TAB) carrier. Electronic assembly and interconnection methods used for various levels of packaging are also described. They include direct-chipattachment (flip chip) process for the MEMS device to the module and bus connection through $\mathrm{TAB}$ carrier to the host computer. Encapsulation material for electronic components was selected for improving the reliability of the module. Localized circuit functionality was needed to conduct temperature compensation and self-calibration. A thin and/or miniaturized profile was required for aerodynamic reasons. Fully assembled hardware in the form of a pressure belt was installed on airplanes and passed two separate flight tests. Data analyses on these flight test results are also included.
\end{abstract}

\section{Introduction}

Many different sensors are needed for aircraft and aerospace systems. They are used primarily to collect data, including static or dynamic pressure, shear stress, acceleration, velocity and noise. Microelectro-mechanical system (MEMS) based sensors can provide significant advantages over conventional ones due to their miniaturized size. Even though MEMS sensors are widely used for single sensor measurements, implementation of sensors in array configurations is rare mainly due to lack of appropriate packaging and suitable communication protocols. In this paper we report a unique multi-MEMS sensor packaging configuration, and its interconnection utilizing MEMS, multichip module (MCM), and tape automated bonding (TAB) technologies. Even though this packaging concept could be applied to many other applications requiring thin profile, our immediate application is the measurement of static pressure during various aircraft. flight load surveys.

\section{MEMS Based 'Pressure Belt'}

During load surveys in flight tests of commercial and military aircraft, arrays of many sensors are required on a common bus. The technology currently used is based on a plastic tube system with remotely located pressure sensors, discrete electronics, and pneumatic system control. It is shown in the lower portion of Figure 1. Some of the pressure measurement locations could be as far as $30 \mathrm{ft}$ away from the pressure sensor location. This approach is cumbersome to install, expensive to operate, and lacks the accuracy needed. It also requires very bulky wiring, which could weigh up to 800 lbs per wing. A new MEMS based 'pressure belt' was proposed to replace the current technology. The new approach as shown in the upper portion of Figure 1 utilizes MEMS sensors located at the point-of-use, and high density MCMs containing associated electronic circuitry. This MCM provides functionality for localized data manipulation including temperature compensation and self-calibration. The MCMs connect to a $\mathrm{TAB}$ tape providing the data and power buses to the host computer.

To be successfully implemented into any airborne hardware, the new system will need to meet various requirements. Two important parameters, which significantly influenced selection of material systems and assembly processes throughout our system and module development, were the thin profile of the belt and measurement accuracy. Other parameters taken into consideration were structural compatibility with the target vehicle, operational temperature range, resistance to moisture and fuel vapor, and other general reliability constraints. The target accuracy of $0.1 \%$ of full scale is about an order better than the typical accuracy possible with the current practices using tubing belt pressure measurement. To meet this objective, module configuration and all the material systems were carefully chosen to minimize any stress being passed onto the sensor diaphragm. Stress can be transmitted from any underlying structure such as the airplane wing or even the MCM substrate itself. One reason why we decided to incorporate MCM substrates with size smaller than 1 in. square is so that it is mountable on curved surfaces. Thin profile (the total thickness less than 0.10 in. from the aircraft surface) was needed for aerodynamic reasons.

When fully implemented, the new system is expected to reduce hardware and installation cost and to improve reliability, while offering an order of magnitude better

\section{(1)}

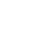

\section{Electronic Components and Technology Conference}


performance. Reduction of installation will have added benefit of process cycle time reduction, which will reduce costs even further.

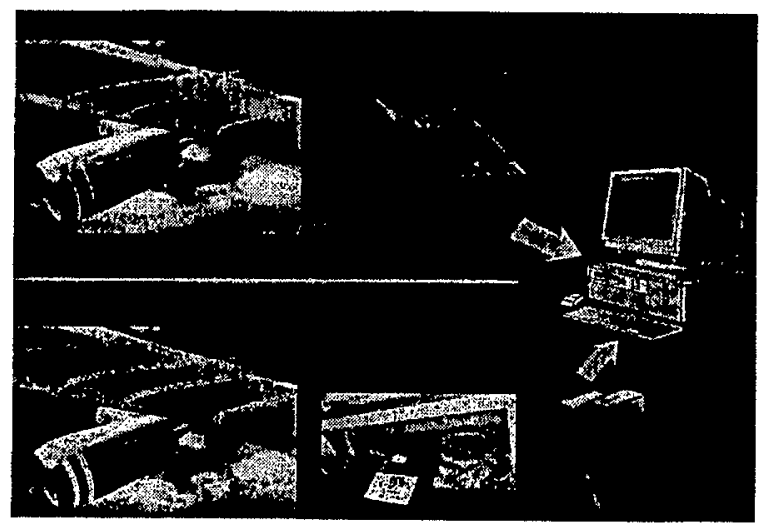

Figure 1. Aircraft flight testing (MEMS vs. Pneumatic Tube)

Figure 2 shows the schematic of the proposed MEMS pressure belt. The first generation of MEMS modules were built and incorporated onto a 'pressure belt' TAB segment to assess viability of their packaging configuration. These units passed two successful aircraft flight tests whose details are described in the last section of this paper.
MEMS Module Substrate and Components

The first generation modules built for this program utilized a bare MEMS device and a commercially available CMOS analog signal conditioner. The conditioner circuits, MCA7707 (equivalent to MAX1457), providing a ratiometric output, were acquired from MCA Technology Inc. Calibration and compensation were controlled by on-module memory (EEPROM.) The memory part used was AT93C66 manufactured by Atmel. The module also needed seventeen passive components to complete the functional circuit.

The MEMS sensor used for the module was a modified version of Endevco's standard product. The sensor was an ion-implanted silicon micro-machined pressure sensor and its diaphragm was designed for a $0-15$ psia pressure range. It incorporated a fully active Wheatstone bridge strain sensing circuit on the internal surface of the diaphragm. This rectangular sensor with .049"x.067" dimensions contained four electrodes and is shown in Figure 3. Two silicon islands in the diaphragm area were formed to help the stress concentrate in the region of interest thus increasing the sensor sensitivity. This being an absolute reference sensor, the reference vacuum was formed with a second silicon support attached to the back of the diaphragm. The support was hermetically glass sealed to the sensor and contained a cavity that was evacuated during the sealing process. Its schematic is shown in Figure 4.

\section{Pressure Belt Segment}

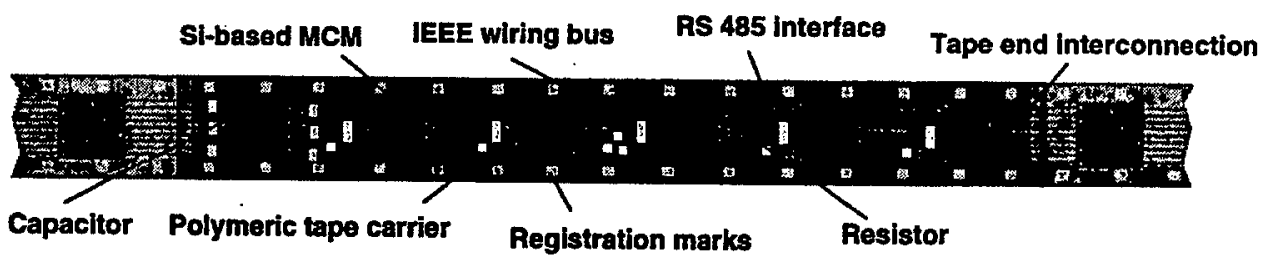

Pressure Belt Cross Section

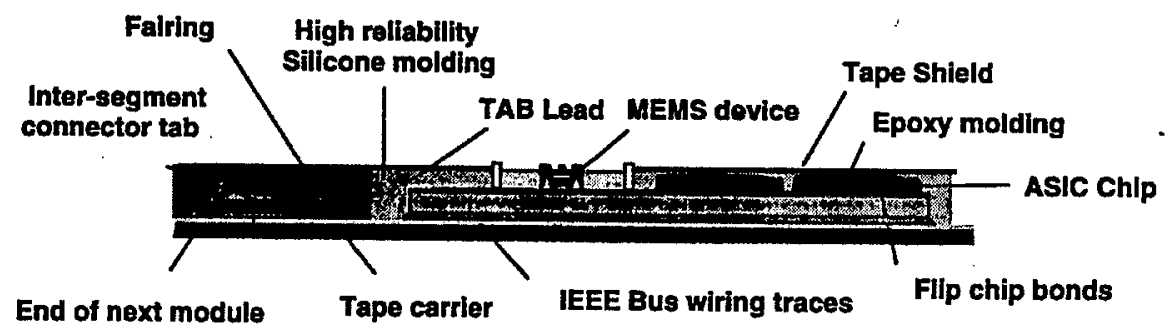

(Vertical scale enlarged for illustration only)

Figure 2. MEMS 'Pressure Belt' schematic

An improvement was made in the reference support to allow flip chip mounting of the MEMS sensor to the silicon MCM using solder bumps or conductive epoxy. This maintains the lowest possible profile since no wires extend above the top of the MEMS device, which is .016 inch thick. The special flip chip support structure contained feedthroughs so that electrical connections could be made from the backside of the sensor assembly. The feedthroughs consisted of etched holes through the reference support. Pad metallization of 
$\mathrm{Ti} / \mathrm{Pd} / \mathrm{Au}$ was then deposited which created electrical bonding sites on the surface of the support and connected them to the pads on the surface of the diaphragm at the bottom of the feedthroughs.

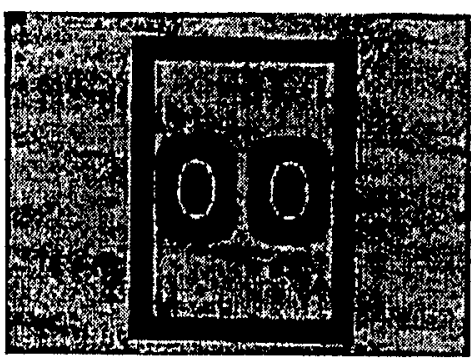

(a) Front side

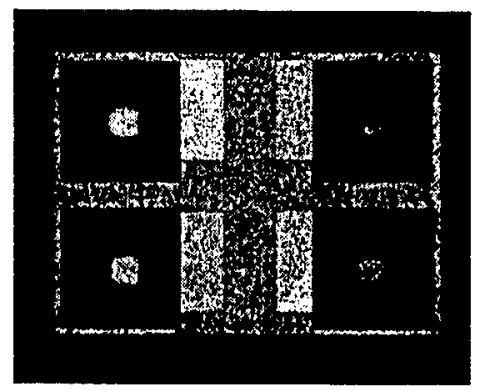

(b) Back side

Figure 3. Photograph of redesigned pressure sensor

The 0.8" $x$ 0.8" MCM substrate was designed and fabricated by Boeing using copper as conductor and photosensitive polyimide as dielectric. Three MEMS sensor sites were allocated to assess the effect of location in the module on the sensor sensitivity. Even though only one MEMS sensor can be connected to the electronic circuitry, high local wiring density was needed since no wiring was allowed underneath the MEMS components. The width and pitch of the conductor lines was $1 \mathrm{mil}$. Due to the relatively thick copper line $(5 \mu \mathrm{m})$ used in this process, line resistance between any two point in our MCM substrate did not exceed 2 ohms. Even though Boeing's MCM structure containing up to 7 layers and its passive embedment potentials have been demonstrated $[1,2$, and 3,$]$ we decided to design a simple structure of two metal conductor and a single dielectric layers as the first generation module. It offered higher yield, higher reliability and lower cost. The module also contained the additional metal films of $\mathrm{Cu} / \mathrm{Ni} / \mathrm{Au}$ suitable for MEMS attachment and wire bondable pads. Further details for design and fabrication of the MCM substrate are also described elsewhere [4.] A MEMS bonding site was made through the polyimide opening and also contained an allocation for a Pyrex glass tube wall surrounding MEMS sensor units. This tube wall was needed not only to provide more aerodynamically acceptable surface profile for sensor accuracy but also to prevent any handling damage to the MEMS sensor during assembly and installation. A single 5" silicon wafer produced $12 \mathrm{MCM}$ units.

Completed MCM wafers were tested electrically for opens and shorts. They were also subjected for burn-in screening tests to eliminate any infant mortality. This consisted of three cycles ranging from room temperature to $250^{\circ} \mathrm{C}$ and seven additional cycles ranging from -55 to $125^{\circ} \mathrm{C}$. These wafers were then diced to produce good MCM units to be used for module assembly.

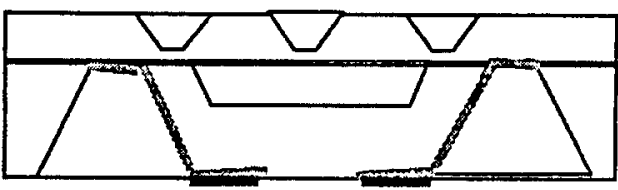

Figure 4. MEMS device schematic

\section{MEMS Interconnection and MCM Assembly}

Figure 5 shows interconnection and attachment materials used in the MEMS module stack. A unique design choice was made with the direct chip attachment (flip chip) of the MEMS device to the MCM substrate. Bonding of MEMS sensor directly onto a silicon oxide coated surface rather than through a polyimide layer became necessary to eliminate any residual stress coming from the cured polyimide and also from thermal coefficient mismatching. Both conductive epoxy and Au-Sn solder materials were tried as flip chip bonding material and produced acceptable results. Selection of two other materials required a careful study for the maximum sensor sensitivity; MCM bonding material to TAB tape and TAB adhesive to airplane wing surface. These three materials, including the selected MEMS flip chip material, were used to effectively isolate any stress that could be transmitted from the vibrations/stressing of the airplane wing to the sensor. At the same time the same three materials are needed to provide robust bond so as to stay securely attached to the airplane body during the flight test. We decided to continue to use a 3M's sticky tape as TAB tape adhesive in spite of its relatively thick profile since it is a proven product for such installations, and provides.clean removal. The 3M 468MP was selected as the MCM mounting adhesive over foamed tape material after a series of stress sensitivity experiments.

Module assembly was conducted by attaching and interconnecting various components onto the $\mathrm{MCM}$ substrate. Various microelectronic assembly processes were used. Choice of processes was somewhat limited, since this first version used only commercially available components. Discrete components were attached to the MCM substrate using Ablestik conductive epoxy while the ICs and the tube wall were bonded using nonconductive epoxy. Gold wedge bonding with low profile wire loop was used for the ICs' electrical connection since they were not available in flip chip compatible wafer form. 


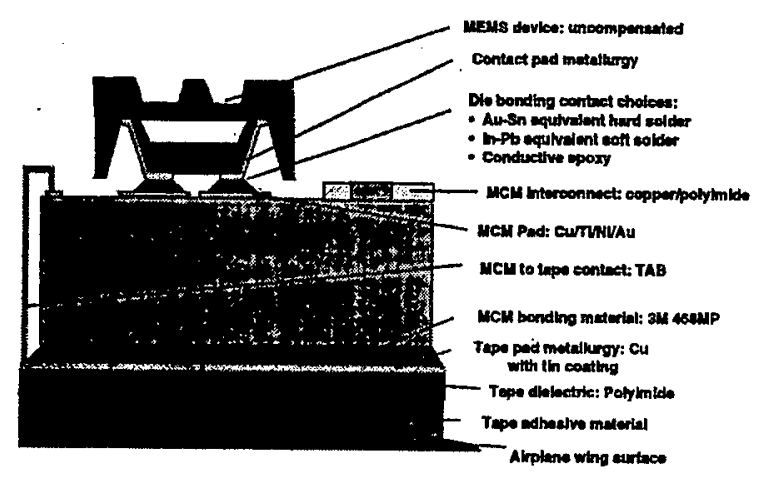

Figure 5. Interconnection and attachment materials used for MEMS MCM Stack

A fully assembled MCM unit and its close-up detail are shown in Figure 6 and 7. These fully populated MCM units were functionally tested using the test procedures developed for process monitoring. It consisted of applying power, establishing serial data communication, and verifying valid signal response from the MCA circuitry. When vacuum was applied to the MEMS sensors, repeatable signal levels were observed within an acceptable range of the anticipated outputs. A very low stress gel, Dow Corning DC Q1-4939, was applied between the MEMS device and the tube wall to protect the base region of the MEMS device and to prevent water invasion. Hysol Dexter 4460 (glob-top) epoxy was applied to protect both ICs. Another functional test was conducted after completing module assembly.

Assembled modules were incorporated onto a TAB carrier to form a 'presure belt' segment. The TAB carrier was $2 \mathrm{mil}$ thick polyimide film in ANSI $70 \mathrm{~mm}$ format with copper conductor wiring. Each segment was 12.342 inch long containing six MCMs. The distance between two MCMs was 2.057 inch. This TAB tape in two-layer construction, without adhesive, was designed by Boeing and procured from $3 \mathrm{M}$.

The tape was tin coated to provide electrical interconnection using either solder reflow or conductive epoxy. Functionally good modules were mounted to the TAB segment using $3 \mathrm{M}$ 468MP adhesive after beam leads had been bent upward through small openings in the polyimde. The beam leads were then side bent using a lead-bending tool, and connected to the peripheral pads on the MCM. The finished functional TAB segment is shown in Figure 8.

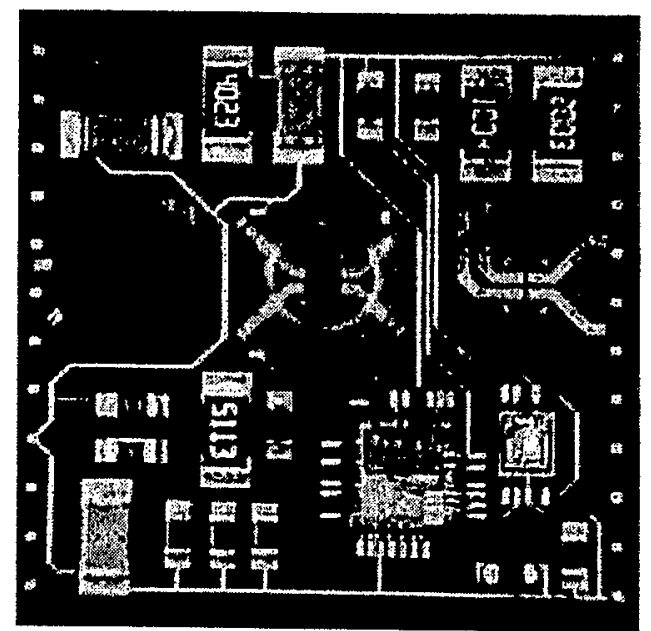

Figure 6. Fully populated MEMS MCM Unit

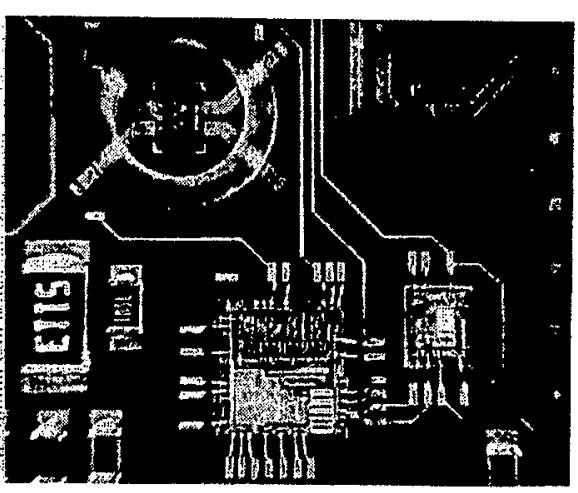

Figure 7. Close-up detail of MEMS and ICs

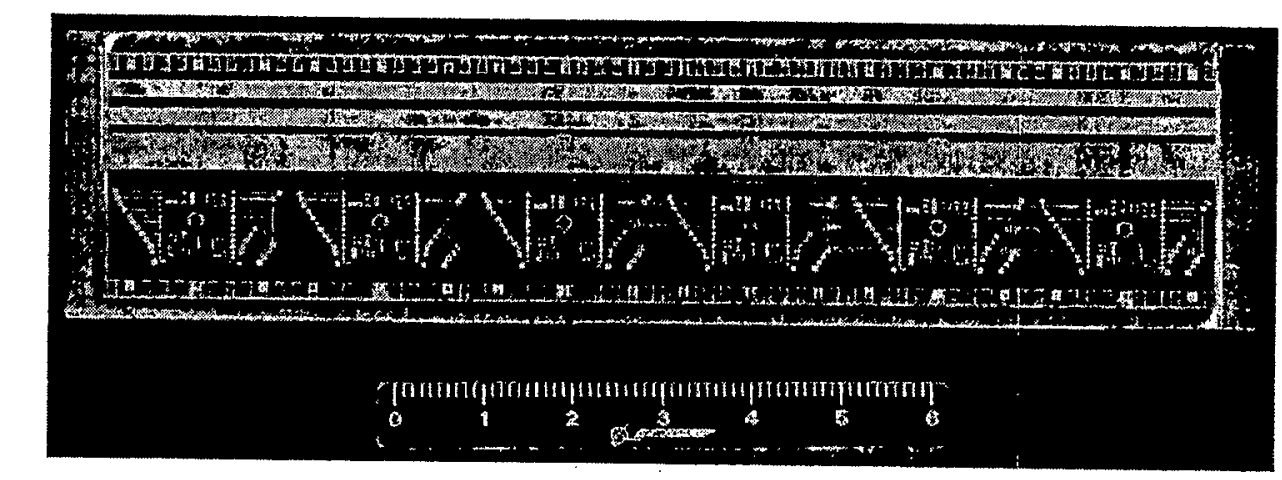

Figure 8. Assembled TAB segment 
Silicone coating of Dow Corning DC1-2577 was applied using a mechanical mold to protect MCM components and beam leads. Figure 9 shows one MCM unit after silicone coating.

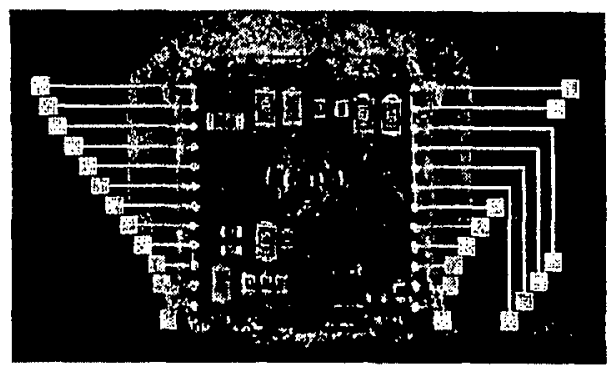

Figure 9. MEMS module after silicone molding

\section{Flight Tests}

Two flight tests were conducted using completed MEMS modules and 'pressure belt' segments to validate their viability as airborne test articles.

The vehicle used for the first flight testing of a completed segment was a Boeing 757-300 undergoing its certification flight testing. The pressure belt was installed in Dec. 98 in the belly section of the aircraft with a fairing attached, close to the landing gear doors. The airplane and its installation site are shown in Figure 10.

A Honeywell PPT transducer (0 to 20 psia range) was installed in an instrumentation rack forward of the main landing gear. A 30 foot plastic tube extended from this pressure transducer's port and terminated at a location adjacent to the prototype pressure beit. The tube was sealed at one end, then a hole was drilled in the tube at a position close to the center of the prototype pressure belt. This arrangement was intended to provide a pressure reference for comparison with the data collected from the 5 operating MCM devices of the prototype pressure belt.

The second flight testing was conducted with a 737 Boeing Business Jet (BBJ) conducting a mini-pressure survey to evaluate wing structural loads during flight. Aviation Partners Inc. installed winglets and instrumented portions of the left wing and left winglet with conventional pressure tube belts. The Boeing Business Jet with the winglets and its installed MEMS modules are shown in Figure 11. In this airplane the number one leading edge slat was instrumented with flush pressure ports located chordwise about 52 inches from the outboard edge of the slat. To measure additional pressures outboard of the flush pressure ports at $35 \%$ of the slat chord, two MEMS modules singulated from the segment that had flown previously with the 757-300. A faring was fabricated into a 6-inch diameter disc placed over the MCM. Metallic tape held the faring down onto the slat surface. Power and signal wires were routed behind and then outboard of the MCM installations, transitioning to instrumentation wiring inside the number one slat. This wiring was terminated at an instrumentation rack, located within the aircraft cabin, to provide power and signal processing for the MCMs. The light blue tape is used to protect the reference ports and the access holes on the fairings. The prototype MCM sensor data was tracking well with pressure data recorded from the flush reference pressure ports, also located at $35 \%$ of the slat chord. The flight loads survey was conducted in Feb. 99 in Mesa, Arizona.

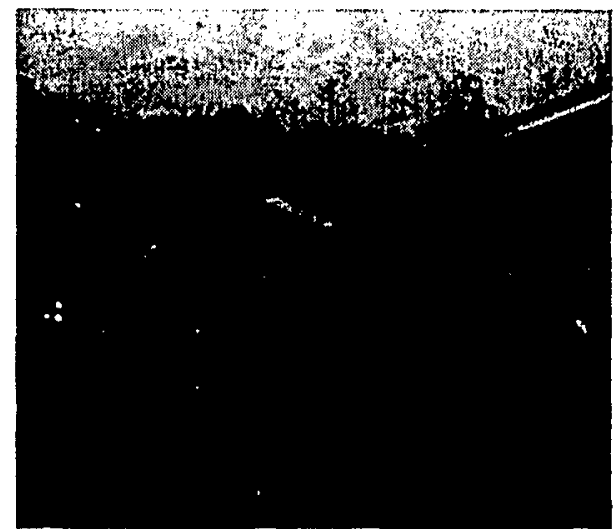

(a) Installation site

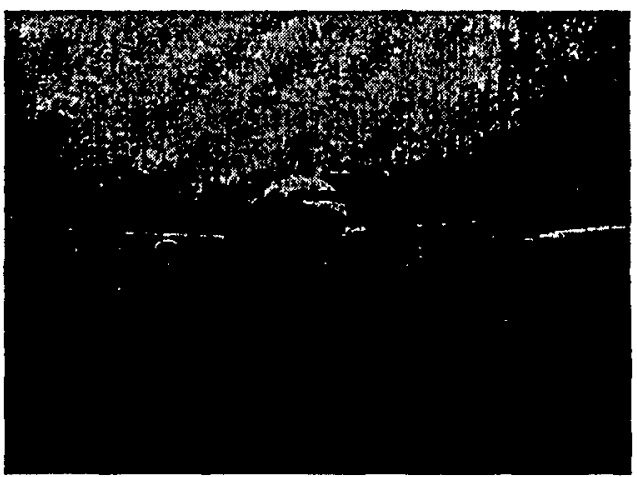

(b) Flight test airplane

Figure 10. Flight test on Boeing 757-300

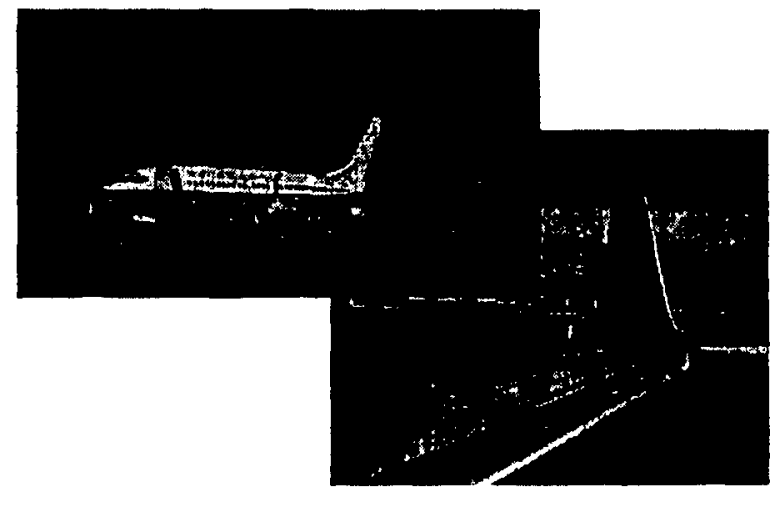

Figure 11. Flight test on Boeing Business Jet 
Test Results

During flight testing, many different parameters are measured and recorded in the main computer. Analysis of a specific parameter requires data extraction from this memory storage.

Selected parameters extracted from the Boeing 757-300 flight test are plotted in Figure 12. Its altitude data shows that the airplane went to cruising at $15,000 \mathrm{ft}$ after three test landings which was maintained for a short period of time.

Thirty data points were collected during this time in the course of 6 seconds ( 5 samples per second data acquisition) and were averaged to properly interpret performance of the MCM devices when directly comparing to the Honeywell PPT reference transducer. The percent of full scale deviations from the averages taken of the PPT pressure readings and the
MCM's are plotted in Figure 13. Except for MCM01, the averaged data samples fell well within the target of $0.1 \%$ of the full scale absolute pressure. Since the conventional tube system has a tendency to average dynamic information, selection of this short time window was the only way we could make a direct comparison between the data from the PPT and 5 operating MCMs. The total volume of the PPT reference pressure port includes both the transducers' internal volume and the volume of the plastic tube. This volume acts as a filter for the dynamic pressures occurring at the source approximately 30 feet away. In contrast, the MEMS sensors are directly exposed to the pressure source and thus capable of responding to dynamic pressure changes in real time (resonant frequency of the MEMS - $180 \mathrm{KHz}$ ).

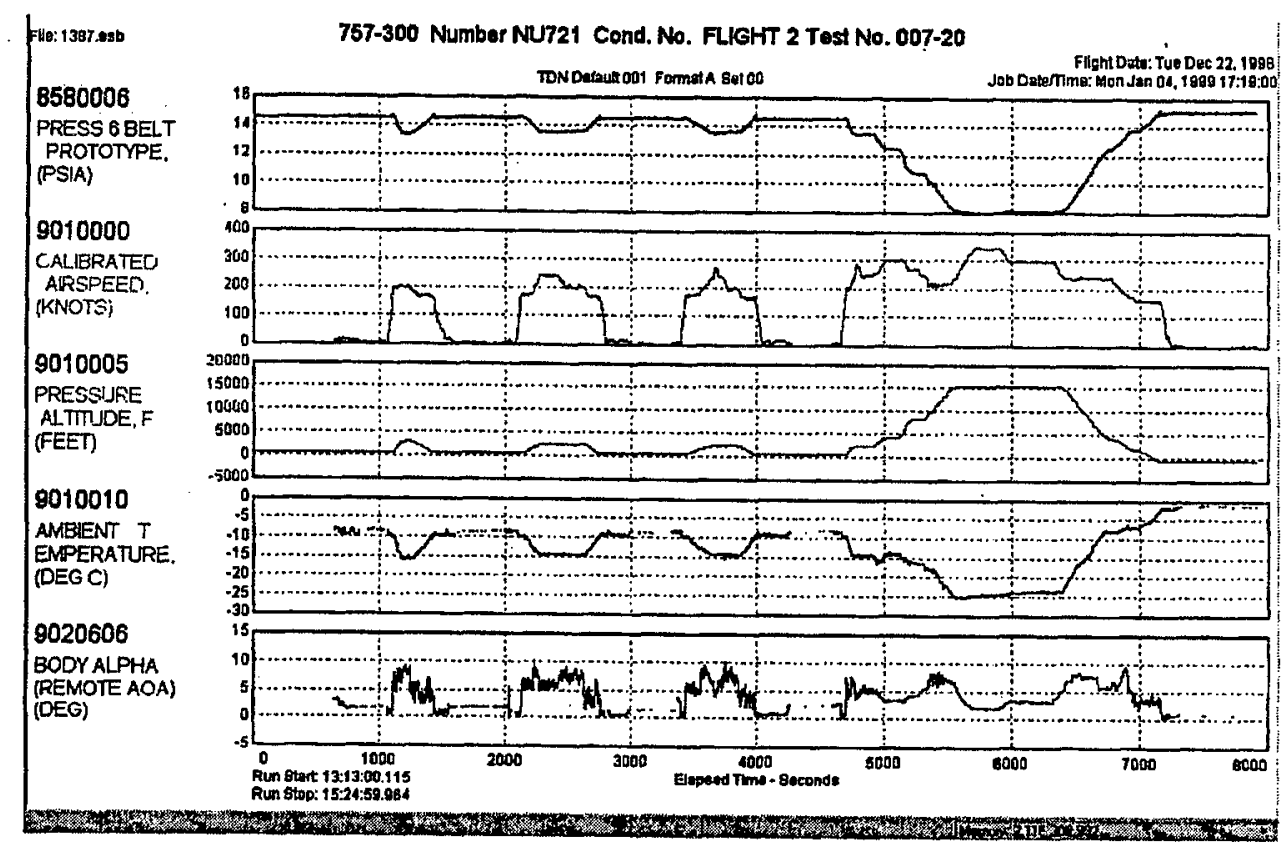

Figure 12. Flight test data from Boeing 757

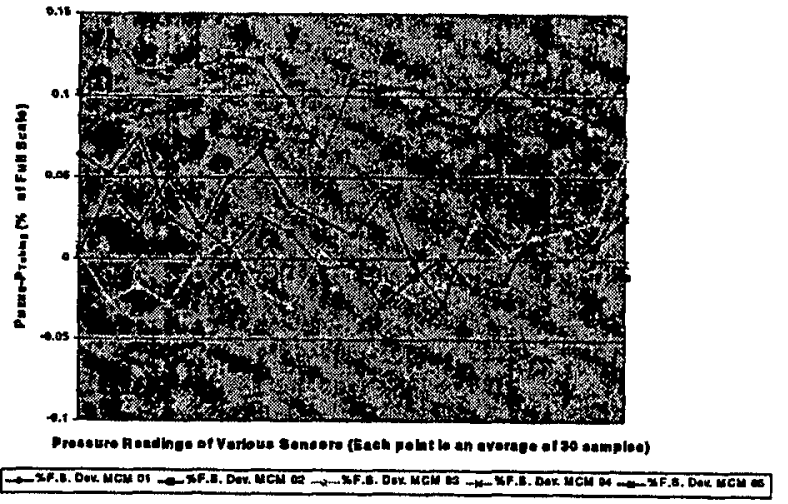

Figure 13. Full scale deviation of pressure measurements
Test data collected during the BBJ flight were also analyzed. Even though direct comparison with the reference data was not possible due to the fact that they were not adjacent to each other, MEMS sensor data followed the exact same trend as the conventional system. It also produced the valuable information that one can use the same article for two consecutive flights. Pressure measurements as a function of time for a "wind-up turn" of the BBJ are shown in Figure 14, while the ones for a "roller-coaster" maneuver are shown in Figure 15. It is believed with a high degree of confidence that the quality of the MEMS sensor data was as good as the data from the 757-300 flight test.

The local speed along the slat cord of the BBJ during cruise conditions was calculated by the aerodynamics staff. The results are shown in Figure 16. 


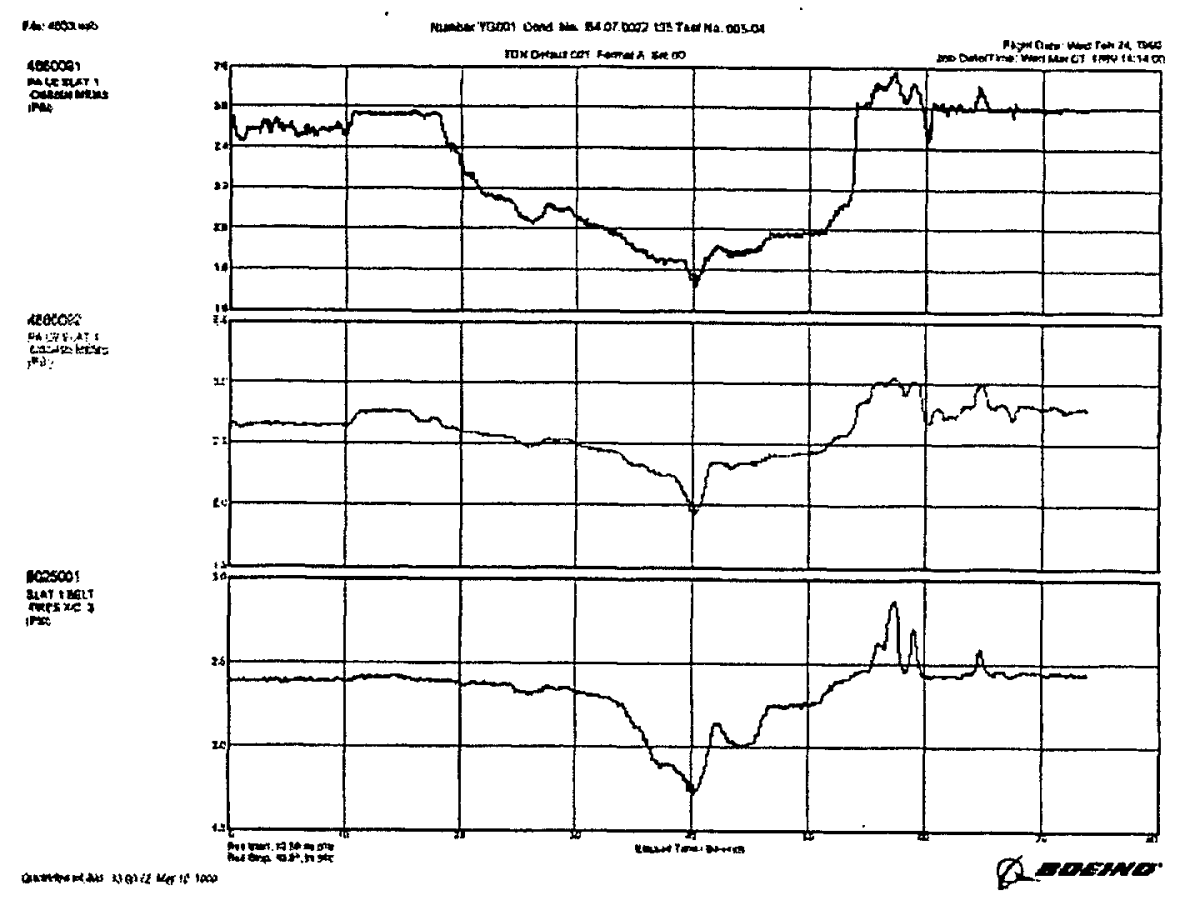

Figure 14. Pressure measurements during "wind-up" turn of the BBJ (Top curve from the reference, two bottom ones from MEMS modules)

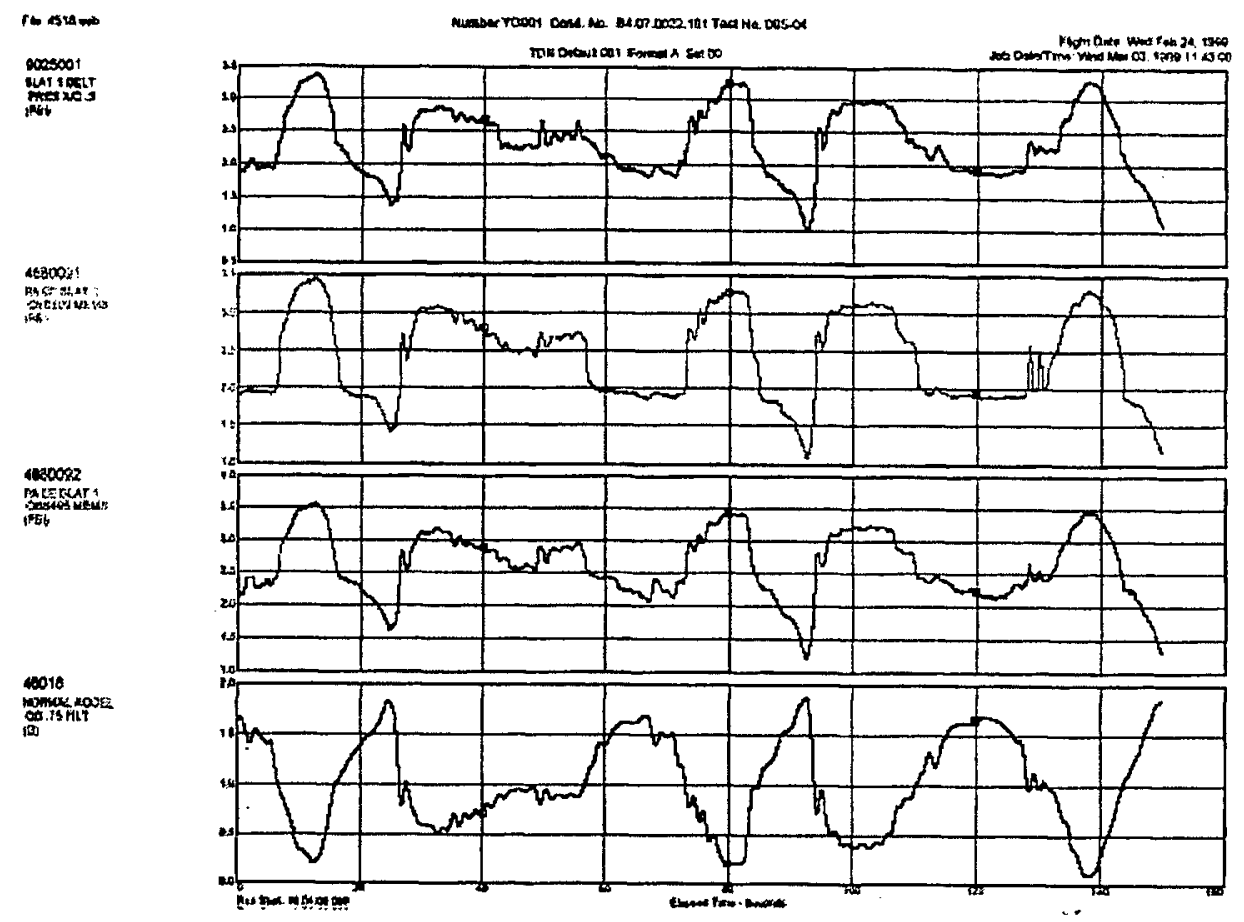

Figure 15. Pressure measurements during "roller-coaster" maneuver (Top curve from the reference, two middle ones from MEMS modules, bottom one indicating aircraft acceleration) 


\section{Discussion}

Packaging configuration of the proposed MEMS module was proven and will remain roughly the same for subsequent versions. A new generation of the 'pressure belt' is being designed with additional improvements. Incorporation of two Boeing designed ASICs with flip chip compatible pads will make the new generation module even lower profile with added electrical functionality for local digitization, robust communication and higher order correction engine. Full electrical functionality for the next generation is has been fully tested at the laboratory level. Results indicate further improvements in sensor accuracy, especially when a polynomial surface fit coefficient correction is incorporated.

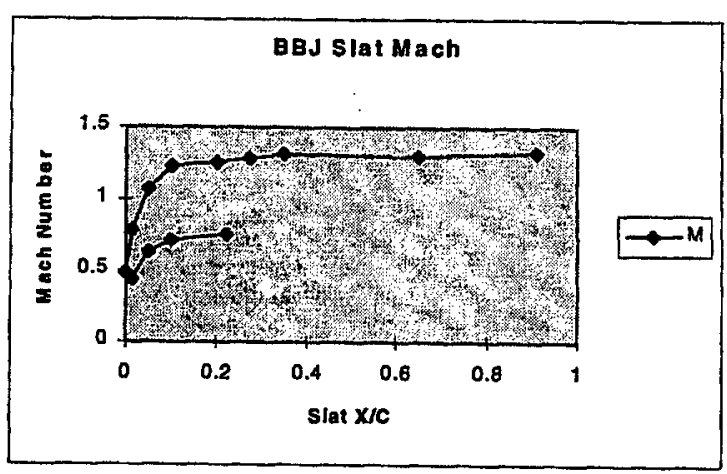

Figure 16: Calculation of the local speed for the BBJ wing slat as a function of the cord distance. The top curve corresponds to the top surface of the wing, while the bottom curve corresponds to the bottom surface of the slat. The zero location is the front of the wing. The location of the MEMS sensors is at $X / C=0.35$ which corresponds to a local speed of 1.3 Mach at the sensor location.

\section{Conclusion}

Development of a MEMS based smart sensor module is reported and its application for the 'pressure belt' used in aircraft flight testing is described. It is used in a multi-sensor system configuration. Its packaging configuration was validated through two consecutive flight tests. When fully implemented, significant cost savings in aircraft flight load surveys are expected since it makes calibration and installation easier and simpler, and provides significantly better measurement accuracy and dynamic information.

\section{Acknowledgments}

This work was partly supported by DARPA/AF Research Laboratory under the agreement F30602-97-2-0099. The authors would like to thank MEMS Pressure Belt IPT members at Boeing and Endevco for their continuous support. We also greatly appreciate $M$. Chisa's support in module assembly. MCM substrate fabrication was contributed by Dr. C-P Chien and his group. We are also grateful to J. Fragala, B. Wilner, and A. Karolys for the MEMS device support.

\section{References}

1. J. Cech et al, "Reliability of Passivated Copper Multichip Module Structures Embedded in Polyimide," IEEE Trans. CHMT, v16, No 7, p.752, 1993

2. K. Coates et al, "Development of Thin Film Resistors for Use in Multichip Modules," Inter. Conf. On Multichip Modules and High Density Packaging, p. 490, 1998

3. N.P. Kim, K.L. Coates, G.G. Kunze, C.P. Chien, and M.H. Tanielian, "Development of Multi-Chip Modules with Integrated Thin Film Passive Elements," Proc. Of $30^{\text {th }}$ International Symp. Of Microelectronics (IMAPS 97), p. 157,1997

4. N. P. Kim et al, "Smart Sensor Multi-Chip Module with Direct-Chip-Attached Micro-Electro-Mechanical System (MEMS) Devices," Proc. Of $32^{\text {nd }}$ International Symposium on Microelectronics (IMAPS 99), p. 484, 1999 


\title{
AIRCRAFT FLIGHT TESTS AND RELIABILITY IMPROVEMENTS OF MEMS PRESSURE SENSOR ASSEMBLY
}

\author{
N.P. Kim, M. J. Holland ${ }^{1}$, C-P. Chien, M. H. Tanielian, J. Wu ${ }^{2}$, and C.P. Wong ${ }^{2}$ \\ Boeing Phantom Works \\ Information, Communication, Sensor \& Electronics Technologies \\ P.O. Box 3999, MS 3W-50, Seattle, WA $98124-2499$ \\ (253) 657-5970, e-mail: namsoo.p.kim@bocing.com \\ 'Boeing Commercial Airplane Group, Flight Test, Seattle, WA \\ ${ }^{2}$ Georgia Institute of Technology, Atlanta, GA
}

\begin{abstract}
Many different sensor types are used in aircraft and aerospace applications in general. During the certification period of a commercial airliner, a loads survey is conducted to collect aerodynamic performance data by measuring the pressure on the surface of the wings. To do this, we developed a unique multi-MEMS pressure sensor system targeted for flight test applications. Here we describe test results collected from two aircraft flight tests using this thin profile 'pressure belt' multisensor assembly. The overall measurement accuracy was close to our targeted $0.1 \%$ of full scale, which is $10 \mathrm{x}$ better than the currently used technology. Lessons learned from these flight tests and approaches to further improve the pressure belt reliability arc also discussed.
\end{abstract}

Keywords: Micromechanical Systems (MEMS), pressure sensor, direct chip attachment (DCA), flip chip, MCM

\section{BACKGROUND}

Many different sensors are needed for aircraft and aerospace systems. They are used primarily to collect data, including static or dynamic pressure, shear stress, acceleration, velocity and noise. Microelectro-mechanical system (MEMS) based sensors can provide significant advantages over conventional ones due to their small size. Even though MEMS sensors are widely used for single sensor measurements, especially in automobile industry, implementation of sensors in array configurations is rare, mainly due to lack of suitable communication protocols. To implement such a multi-sensor system in any application, all the components including the hardware, firmware, and software need to be developed. We have reported earlier the development of a smart sensor multichip module (MCM) containing MEMS-based pressure transducers (1.) The module contained a bare MEMS device, and a CMOS Analog Signal Processor ASIC, an EEPROM and a number of passives. $A$ unique assembly, which we refer to as a 'pressure belt', was developed using a large number of these smart modules. Even though the packaging approach may be appropriate to other applications requiring thin profilc, our target application was the measurement of static pressures during aircraft flight load surveys. In this paper we describe the packaging configuration of the pressure belt, and data collected from two flight tests using Boeing a 757-300 and a 737-BBJ aircraft. Lessons learned during these tests helped us improve our design and ultimately the assembly and packaging reliability.

\section{MEMS SENSOR 'PRESSURE BELT' AND SYSTEM REQUIREMENTS}

During load surveys in flight tests of commercial and military aircraft, it is desirable to measure the pressure on the surface of the wings and nacelles for every few inches. The technology currently used for this purpose is based on a plastic tube system with remotely located pressure sensors, discrete electronics, and pneumatic system control. This approach, shown in the lower portion of Figure 1, is cumbersome to install, expensive to operate, and lacks the accuracy needed. A new MEMS based 'pressure belt' was developed to replace the current technology. The new approach is shown in the upper portion of Figure 1. It utilizes MEMS sensors located at the point-of-use on high density MCMs, which contain associated electronic circuitry (1.) This MCM provides functionality for localized data manipulation, including temperature compensation and selfcalibration. The MCMs connect to a Tape Automated Bonding (TAB) tape, which provides interconnection for data and power to the host computer. Figure 2 shows a schematic drawing of the proposed MEMS pressure belt whose construction is described in detail in the later sections.

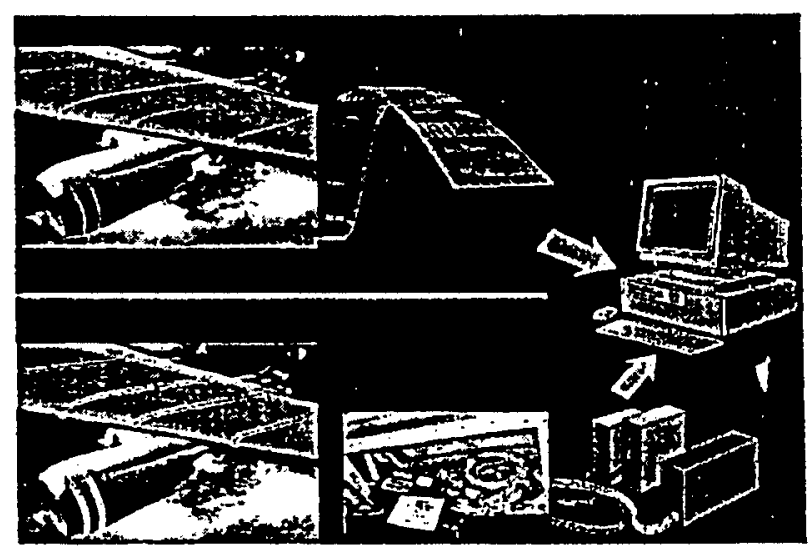

Figure 1. Aircraft flight testing (MEMS vs. Pneumatic Tube) 
To be successfully implemented into any airborne hardware, the new system will need to meet various requirements. Two important parameters, which significantly influenced the selection of material systems and assembly processes throughout our development, were the thin profile of the belt and the measurement accuracy. Other parameters taken into consideration were structural compatibility with the target vehicle, operational temperature range, resistance to moisture and fuel vapor, and other general reliability constraints. The target accuracy of $0.1 \%$ of full scale over a temperature range from $-551080^{\circ} \mathrm{C}$ is about an order better than the typical accuracy possible with the current practices using tubing belt pressure measurements. To meet this stringent accuracy objective, module configuration and all the material systems were carefully chosen so as to minimize any stress transmitted to the MEMS sensor, which could result in an erroneous value. Sources of stress in this environment are the underlying structure such as the airplane wing or even thc MCM substrate itself. The reason we decided to use MCM substrates 1 in $^{2}$ in size was capable of mounting on curved surfaces. The overall thin profile (total thickness less than 0.10 in. from the aircraft surface) was needed for aerodynamic reasons. Finally, the MCM substrate was chosen to be Si so that it matched the thermal expansion coefficient of the sensor itself.

\section{Pressure Belt Segment}
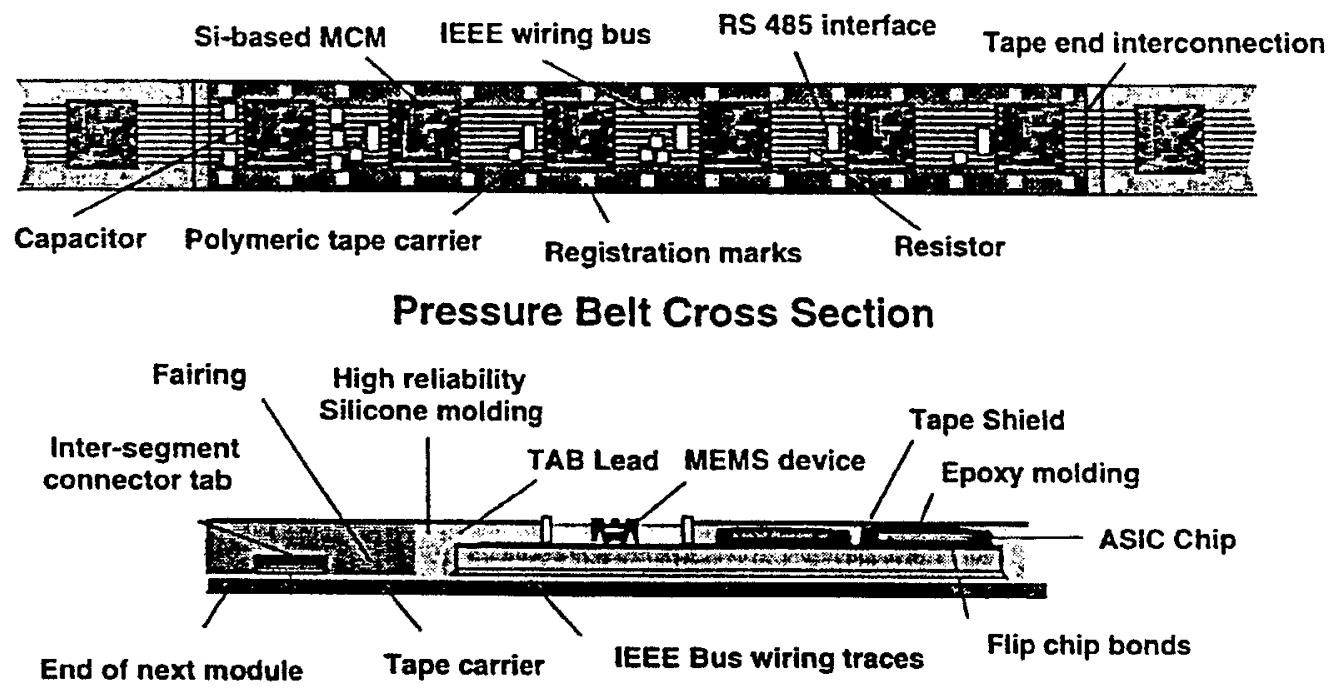

(Vertical scale enlarged for illustration only)

Figure 2. MEMS 'Pressure Belt' schematic

\section{SMART MEMS MODULE COMPONENTS}

Components used for the first generation modules built for this program included a bare MEMS device and a commercially available CMOS analog signal conditioner. This part, MCA7707 (equivalent to MAX1457), providing a ratiometric output, was acquired from MCA Technology Inc. Calibration and compensation was controlled by an on-module memory (EEPROM) chip. The memory part used was AT93C66 manufactured by Atmel. The module also needed seventeen passive components to complete the functional circuit.

The MEMS sensors were supplied by Endevco with some modification in its packaging format. The micro-machined sensor has a fully active Wheatstone bridge strain sensing circuit on a thin Si diaphragm. formed through ionimplantation. The diaphragm is designed for 0-15 psia pressure range opcration. This rectangular sensor contained four VOs as shown in Figure 3. Two silicon islands in the diaphragm area as shown in Figure 3a were formed to help the stress concentrate in the region of interest thus increasing the sensor sensitivity. The reference vacuum, which provides an absolute reference for the sensor, is shown in Figure $3 \mathrm{c}$. The reference support was hermetically glass sealed to the sensor to form a cavity that was evacuated during the sealing process. This reference support has access via feedthroughs, which allow flip chip mounting of the MEMS sensor to the silicon MCM using solder bumps or conductive epoxy. This configuration provided the lowest possible profile and the best reliability since no wires extend above the top of the MEMS device. The feedthroughs consisted of etched holes through the reference support. Pad metallization was then deposited which created electrical bonding sites.

\section{MEMS MCM SUBSTRATE}

The module substrate with a size of $0.8^{\prime \prime} \times 0.8^{\prime \prime}$ was designed and fabricated using copper as conductor and polyimide as dielectric. Boeing's fabrication process has been described in detail earlier (1-4.) Three MEMS sensor 
sites were allocated to assess the effect of sensor to its sensor sensitivity. No wiring was allowed underneath MEMS components. The width and pitch of the conductor lines was $1 \mathrm{mil}$. Figure 4 shows the cross section of the prototype module.

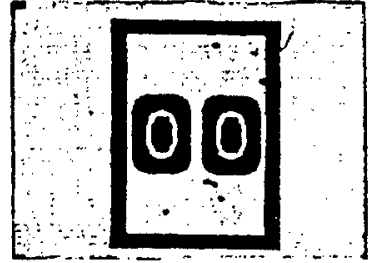

(a) Front side

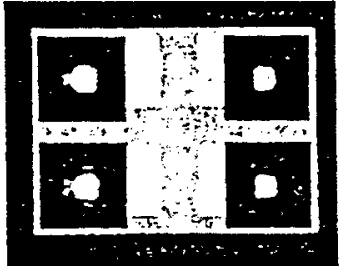

(b) back side

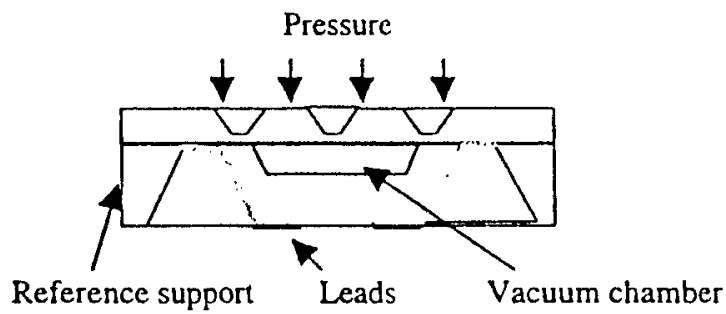

(c) schematic

Figure 3. Redesigned pressure sensor

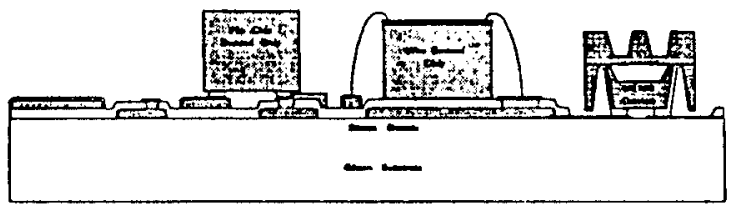

Figure 4. MCM schematic cross-section

MEMS MODULE AND SEGMENT ASSEMBLY

The MEMS sensor was directly bonded onto a silicon oxide coated surface on the MCM rather than through a polyimide layer to eliminate any residual stress coming from the cured polyimide and also from thermal expansion coefficient mismatching. Both conductive epoxy and AuSn solder materials were tried for flip chip bonding and produced acceptable results. Two other materials were selected carefully to maintain the maximum sensor sensitivity: the MCM bonding material to TAB tape and the $\mathrm{TAB}$ adhesive to airplane wing surface. All materials, including the MEMS flip chip bonding material, were chosen to isolate stresses that arise due to the vibrations/stressing of the airplane wing while at the same time providing a robust bond for secure attachment. We decided to use a $3 \mathrm{M}$ "speed" tape as the adhesive between the TAB tape and the airplane wing since it is a proven product for such installations, and provides clean removal. The $3 \mathrm{M} 468 \mathrm{MP}$ was selected as the MCM mounting adhesive to the $T A B$ tape after a series of evaluation experiments.
The other components were attached onto the substrate using standard microclectronic assembly processes. A glass ring was attached around the MEMS device to act as a dam for the protective coatings. Discrete components were attached to the MCM substrate using Ablestik conductive epoxy while the ICs and the glass ring were bonded using nonconductive epoxy. Gold wedge bonding with low profile wire loop was used for the ICs' electrical connection since they were not available in flip chip compatible format. A fully assembled protolype version of the MCM unit is shown in Figure 5. A very low stress gel, Dow Corning DC Q1-4939, was applied between the MEMS device and the tube wall to protect the base region of the MEMS device and to prevent water invasion. Hysol Dexter 4460 (glob-top) epoxy was applied to protect both ICs.

Assembled modules were incorporated onto a TAB carrier to form a 'pressure belt' segment. The TAB carrier was 2 mil thick polyimide film in ANSI $70 \mathrm{~mm}$ format with copper conductor wiring. Each segment was 12.342 inch long containing six MCMs. This TAB tape with tin coating was designed by Boeing and procured from $3 \mathrm{M}$. Functionally good modules were mounted to the TAB segment using $3 \mathrm{M} 468 \mathrm{MP}$ adhesive after beam leads had been bent upward through small openings in the polyimide. The beam leads were then side bent and connected to the peripheral pads on the MCM. The finished functional TAB segment is shown in Figure 7. Silicone coating using Dow Corning DC1-2577 was applied to protect the MCM components and beam leads. Figure 8 shows one MCM unit after silicone coating.

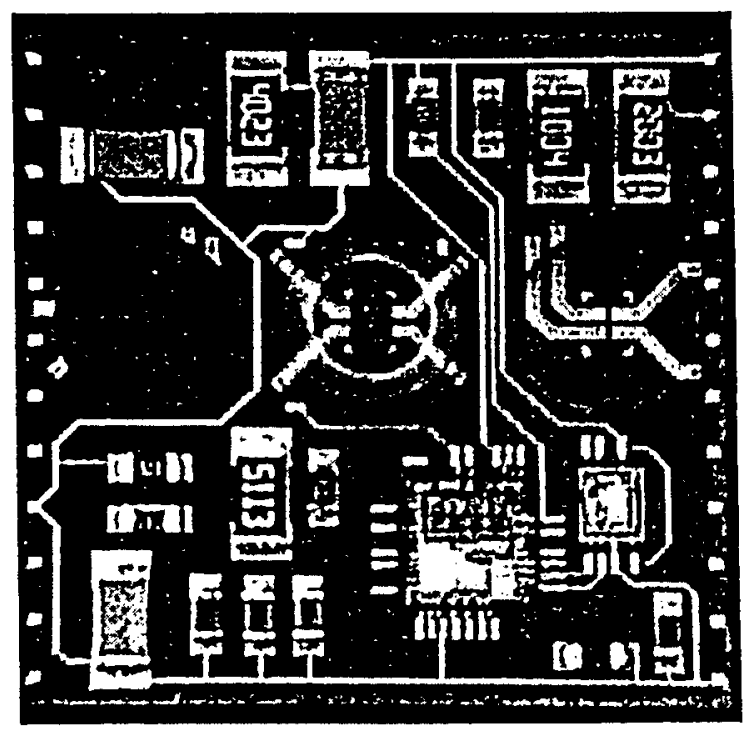

Figure 6. Fully populated MEMS MCM Unit

\section{TEST FLIGHTS AND LESSONS LEARNED}

Two night tests were conducted using completed MEMS modules and 'pressure belt' segments to validate their viability as airborne test articles. 


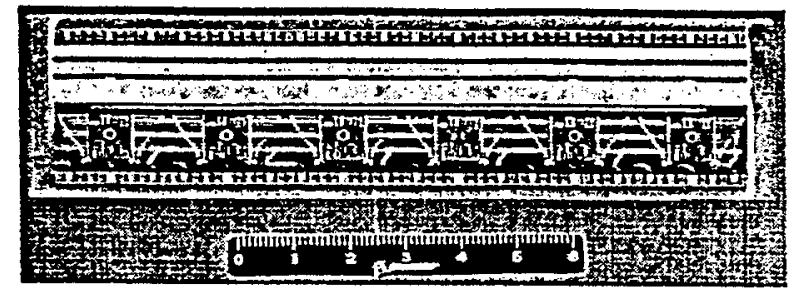

Figure 7. Assembled TAB segment

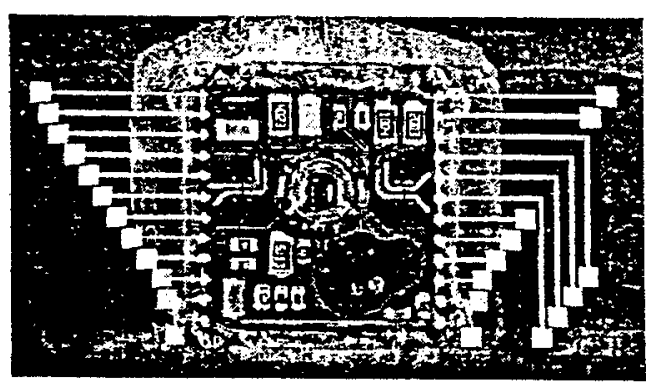

Figure 8. MEMS module after silicone molding

The first flight test of a completed segment was conducted using a Boeing 757-300 undergoing its certification flight testing. The pressure belt was installed in the belly section of the aircraft with a fairing attached, close to the landing gear doors. A Honeywell PPT transducer (0 to 20 psia range) was also installed in an instrumentation rack forward of the main landing gear to provide a pressure reference for comparison with the data collected from the MCM devices of the prototype pressure belt.

Many different parameters were measured and recorded in the main computer during flight testing. Selected data from the Boeing 757-300 flight test are plotted in Figure 9. In this figure we see the airplane landing and taking off three times before it takes off and reaches an altitude of $-15,000$ ft. To compare the data measured by the MEMS sensors to the reference tubing system using the Honeywell PPT one has to choose a flight condition where pressure variations are minimal, in order to eliminate any dynamic changes arising from differences in the time constants between the two systems. Thirty data points were collected during 6 seconds ( 5 samples per second data acquisition) for a stable cruise condition and were averaged to properly interpret the performance of the MCM devices to the Honeywell PPT reference transducer. The percent of full scale deviation from the averages taken of the PPT pressure readings and the MCMs are plotted in Figure 10. Except for MCM01, the averaged data samples fell well within the target of $0.1 \%$ of the full scale absolute pressure measurement accuracy. Since the conventional tube system has a tendency to average dynamic information due to the long length of the plastic tube $(-30 \mathrm{ft}$ ) selection of this short time window was the only way we could make a direct comparison between the data from the PPT and $5 \mathrm{MCMs}$.
This also demonstrates one of the advantage of the MEMS pressure belt vs. the conventional system. The MEMS system is directly exposed to the pressure source and is capable of responding to dynamic pressure changes in real time (resonant frequency of the MEMS $-180 \mathrm{KHz}$ ), while the conventional system tends to filter all that information.

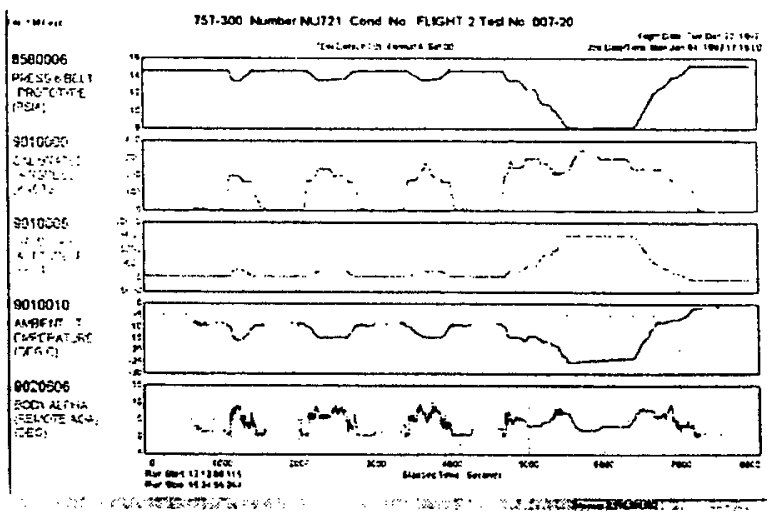

Figure 9. Flight test data from Boeing 757

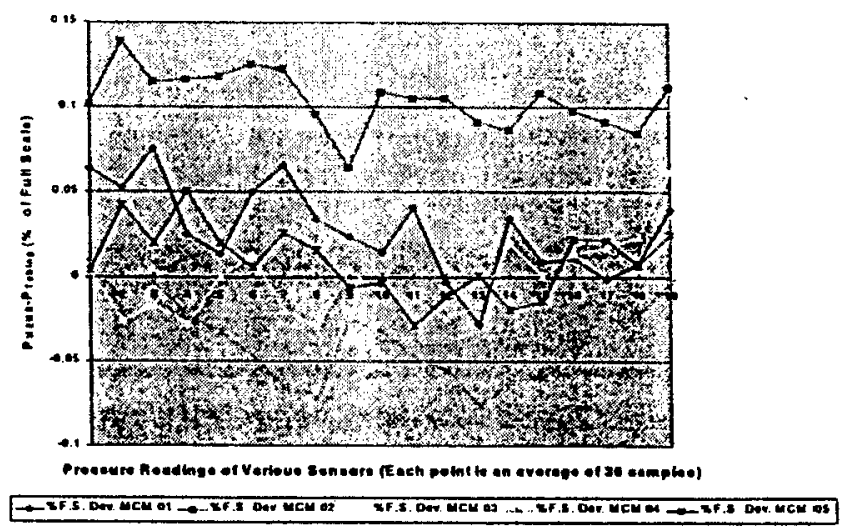

Figure 10. Full scale deviation of pressure measurements

The second flight test was conducted with a 737 Boeing Business Jet (BBJ) which was conducting a mini-pressure survey to evaluate the effect of winglets on airplane performance. The number one leading edge slat was instrumented with flush pressure ports located chordwise about 52 inches from the outboard edge of the slat. To measure additional pressures outboard of the flush pressure ports at $35 \%$ of the slat chord, two MEMS modules were attached, singulated from the segment that had flown previously with the 757-300. A faring was fabricated into a 6-inch diameter disc placed over the MCM. Metallic tape held the faring down onto the slat surface. Power and signal wires were routed behind and then outboard of the MCM installations, transitioning to instrumentation wiring behind the slat.

Even though direct comparison of the MEMS sensor 
readings to the reference data during the $\mathrm{BBJ}$ flight test was not possible duc to the fact that they were not adjacent to each other, the MEMS sensor data followed the exact same trend as the conventional system. It also demonstrated that onc can use the same article for two different installations. Pressure measurements as a function of time for a "wind-up turn" of the BBJ are shown in Figure 11, while the ones for a "roller-coaster" maneuver are shown in Figure 12. The local speed along the slat cord of the BBJ during cruise conditions was calculated and the results are shown in Figure 13.

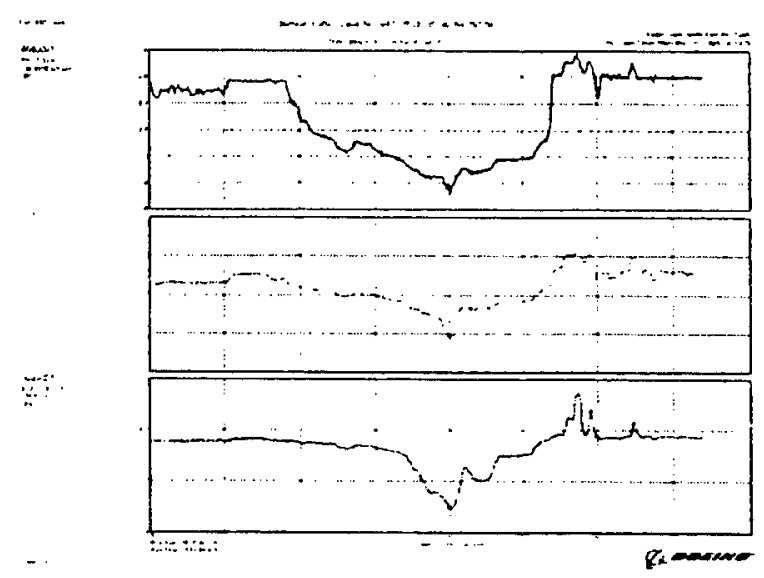

Figure 11. Pressure measurements during "wind-up" turn of the BBJ (Top curve from the reference, two bottom ones from MEMS modules)

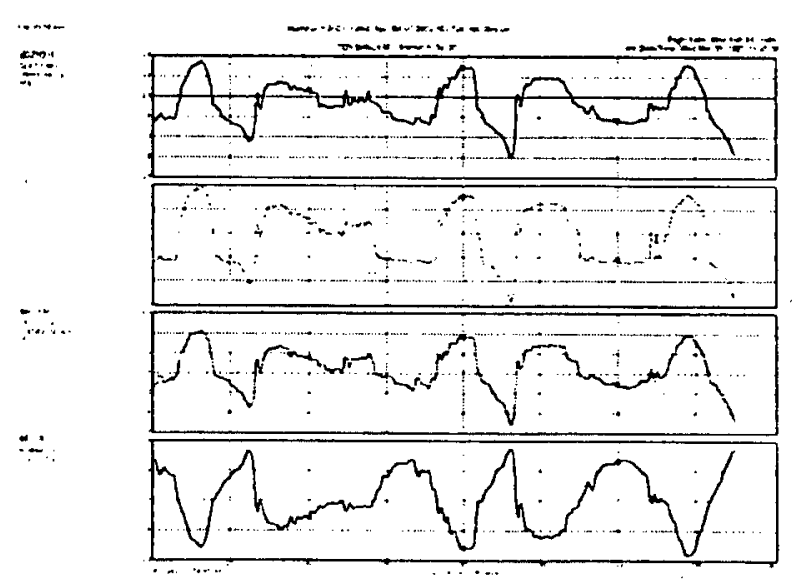

Figure 12. Pressure measurements during "roller-coaster" maneuver (Top curve from the reference, two middle ones from MEMS modules, bottom onc indicating aircraft acceleration)

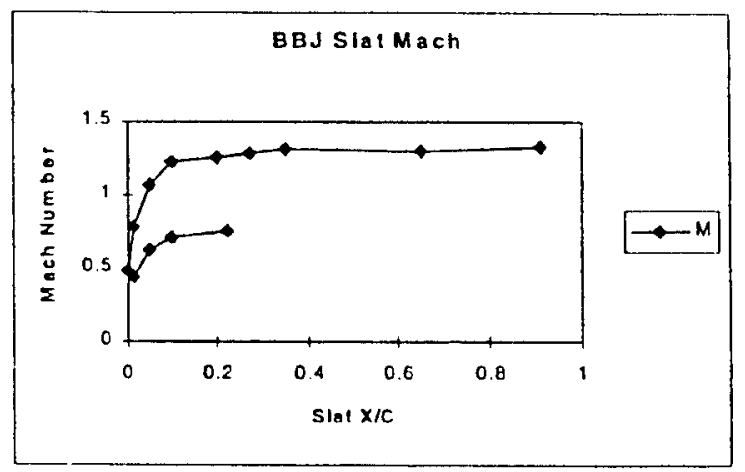

Figure 13. Calculation of the local speed for the BBJ wing slat as a function of the cord distance. The top curve corresponds to the top surface of the wing, while the bottom curve corresponds to the bottom surface of the wing. The zero location is the front of the wing. The location of the MEMS sensors is at $\mathrm{X} / \mathrm{C}=0.35$, which corresponds to a local speed of $1.3 \mathrm{Mach}$ at the sensor location.

After many flight segments, the two MEMS units ceased operation during the return flight of the BBJ. Both units failed approximately at the same instance. From the flight test recorder and pilot's report, it was concluded that the failure occurred during heavy rain and very large turbulence. The acceleration, the altitude, and the air speed are shown in Figure 14 against elapsed time along with the pressure sensor data produced by the two MEMS/MCM units. As it is apparent from these data, the failure corresponds to an increase of the acceleration spectrum of the airplane, indicating severe turbulence. Since the accelerometer readings were taken in the airplane cabin, it is reasonable to assume that the acceleration of the wing slat was significantly larger at the sensor location when the failure occurred.

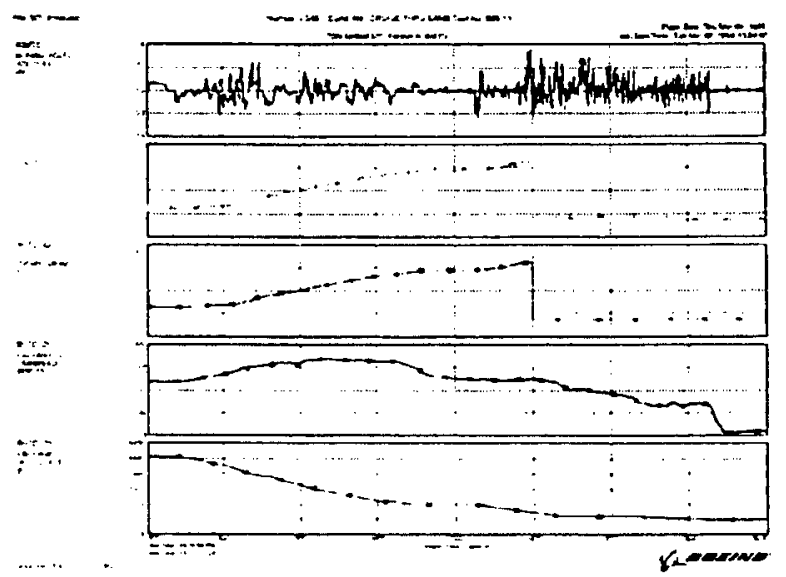

Figure 14. MEMS unit failure (from top, curves for acceleration, MEMS I pressure, MEMS 2 pressurc, air speed, and altitude) The airplane was in its final descent in Seattlc. 
After carefully removing the two units, we conducted failure analyses using the scanning electron microscope and electrical measurements. Resistance measurements at different locations indicated that the failure was associated with the MEMS device. SEM and optical microscopic inspection revealed a fractured diaphragm in the MEMS device. Full functionality of both failed MCM units was restored when both failed units were reworked by carefully replacing the MEMS devices with new, functioning ones. Figure 15 shows the diaphragm region of the failed unit. The cause of this fracture is believed to be over-pressure caused by the direct impact of rain drops with high momentum.

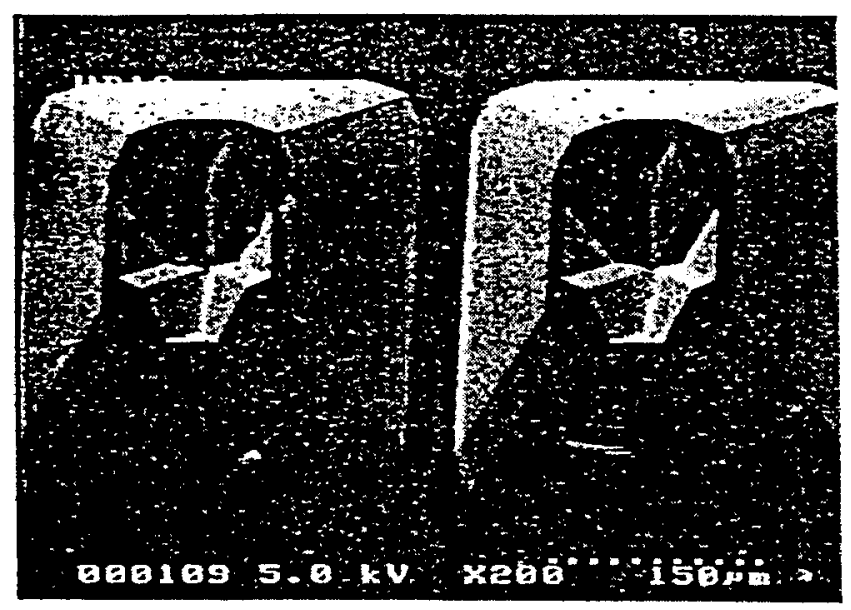

(a) Crack visible in the diaphragm area

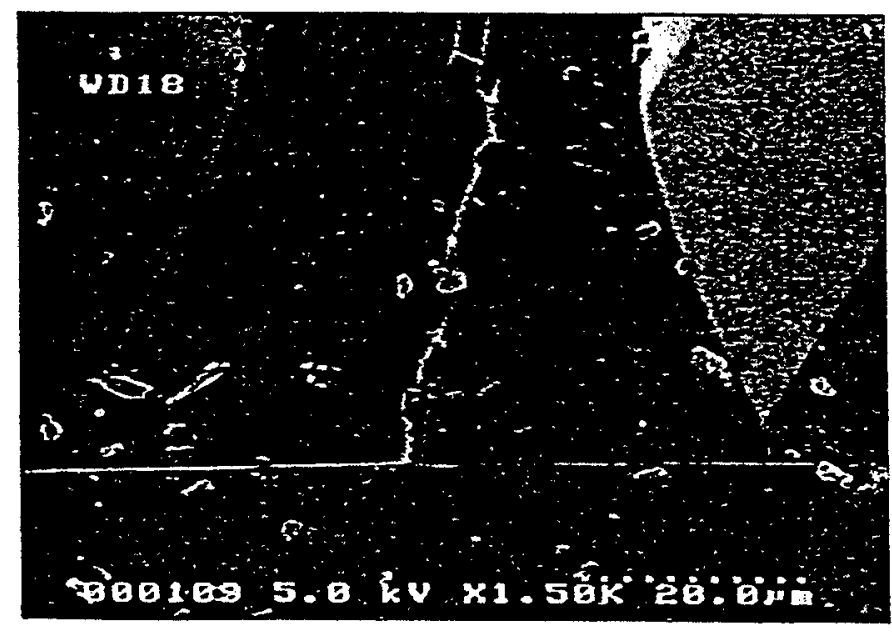

(a) Central region with higher magnification

Figure. 15 SEM of the failed MEMS unit

\section{Reliability Improvements}

Two major improvements have been incorporated to enhance the reliability of the pressure belt; incorporation of the protection shield and improvement in protective coatings.
The protection shicld was needed to prevent the same mode of failure observed with BBJ flight test samples. It consists of flat metal shield mounted on top of our existing glass ring. It is circular and contains small holes $(10 \mathrm{mil}$ diameter) in the periphery of the shield with the central region blocked to protect the diaphragm area of the MEMS device from direct impingement. Two separate experiments were conducted to test the effectiveness of this shicld

The first experiment was a water jet experiment. The goal of this experiment was to assess the effectiveness of the shield for raindrop over-pressure. Two protolype units were prepared with only the MEMS device and glass ring installed. The metal shield was applied to one unit (as shown in Figure 16) while the other unit was kept as control. Both units were subjected to a consistent water jet stream applied normally to the sample using identical experimental procedures. Both units were physically inspected for diaphragm integrity and their bridge resistances were measured before and after water jet exposure. The control (unshieided) sample suffered complete destruction of the diaphragm area indicating that the experimental condition was worse than the flight condition when the failure occurred. The shielded unit on the other hand survived the water jet experiment and its bridge resistances showed very little difference. The shielded unit was subjected to additional water jet stream applied at a $45^{\circ}$ angle to the sample. It survived this additional water jet exposure, which led to the conclusion that the shielded unit could survive the rainstorm environment much worse than the one encountered with our previous units.

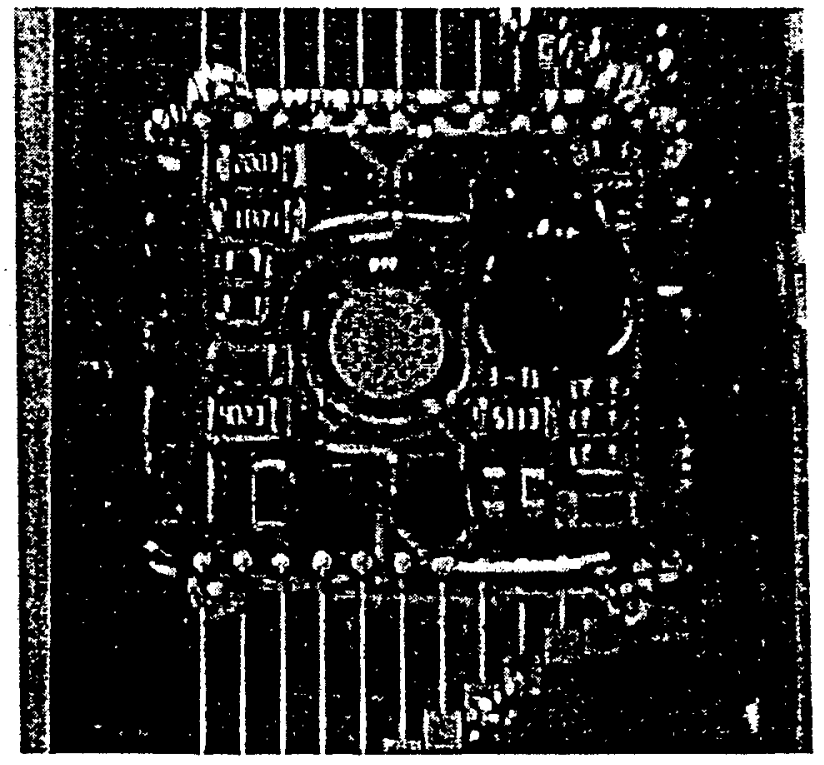

Figure 16. MEMS unit with the protection shield applied over the MEMS device

The second experiment conducted was a trapped-moisture freezing experiment. The goal of this experiment was to address the concern associated with volume expansion 
when moisture trapped inside the glass ring cavity becomes frozen. Since the shield has only tiny pressure holes, there was some concern that moisture may not fully evaporate or escape thus remaining trapped. Four samples werc prepared; two with the metal shield covered and two unshielded controls. The cavity of the one unshielded unit (labeled as \#2) was filled with water while the other unshiclded unit was kept as control (\#1). Among two shielded units, one sample (\#4) was immersed in water for $10 \mathrm{~min}$. while the other (\#3) was kept as is. This was donc to simulate exposure to rainstorm prior to takeoff. All four units were loaded in the temperature/humidity chamber and subjected to $100 \%$ humidity simulating continuous rain/mist environment. After 20 minute soaking in $100 \%$ relative humidity at room temperature, the chamber temperature was ramped down $10-55^{\circ} \mathrm{C}$ at a cooling rate of $-3.5^{\circ} \mathrm{C} / \mathrm{min}$. This cooling rate was selected to simulate the altitude temperature change typically encountered with the commercial aircraft during ascent. After soaking at $-55^{\circ} \mathrm{C}$ for $20 \mathrm{~min}$, the chamber temperature was ramped up to room temperature with a heating rate of $3.5^{\circ} \mathrm{C} / \mathrm{min}$. All samples were physically inspected and their bridge resistances were measured and recorded before and after the trapped moisture freezing experiment. At the beginning, we planned to focus on the results of three samples $(\# 1,2,3)$ expecting failure of \#4 sample. To our surprise, all four samples survived this freezing experiment. Little change in bridge resistances was observed. It seems that even though the shielded unit (\#4) was immersed in water, it still contained a trapped air pocket since the pressure holes are small so water surface tension may not allow any water penetration into the cavity where the MEMS device is located. This trapped air appears to have prevented any damage during the freezing experiment.

The second improvement we are making is the use of protective coatings for the bare chip devices. We already know that even when the MEMS sensors were damaged the rest of the MCM modules remained functional. However, it is clear that from a manufacturing point of view it is desirable to be able to replace the bi-layer coating with a single layer. Even if this proved impossible, is was still desirable to be able to replace the top silicone layer with an alternate material, which had physical properties similar to silicone, but having an increased resistance to jet fuel.

To replace the current bi-layer system, commercially available low stress conformal coating materials were evaluated for low residual stress, low thermal expansion coefficient, good planarity and chemical resistance. Materials recommended by manufacturers included EpoTek 301-2FL, Epo-Tek 301, OE107-1 (Epoxy Technology), FP4651 (Dexter Hysol), Abelstik 7900, 7900H-Dam, 7950-Fill (Abelstik). The residual stress of materials is determined by their modulus and $\triangle C T E$ combined with the variation of temperaturc $\left(\sigma=\int_{T_{1}}^{T_{2}} E \cdot \Delta C T E d t\right.$ ), experiments conducted on these materials found that the residual stresses of all these candidates did not mect our requirements. To improve stress characteristics, we tried a CTBN modified epoxy combincd with low molecular weight anhydride and diluent epoxy using a tertiary amine as catalyst. Two kinds of diluent epoxies (A and B) were used to change the viscosity of the formulated samples. Figure 17 and 18 show the storage modulus with the variation of type $A$ and $B$ diluents. Even though we found that the storage modulus values of some formulations could be comparable to the one with DC1-2577, none of those maintained its low value at low temperatures, such as $-55^{\circ} \mathrm{C}$, which is typical of the cruise condition environment.

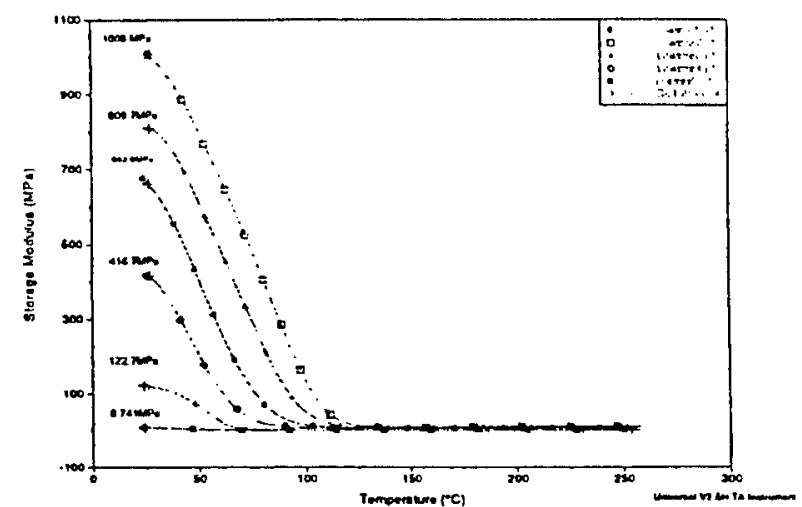

Figure 17. Storage modulus varied with percentage of type A epoxy diluent (DMA test)

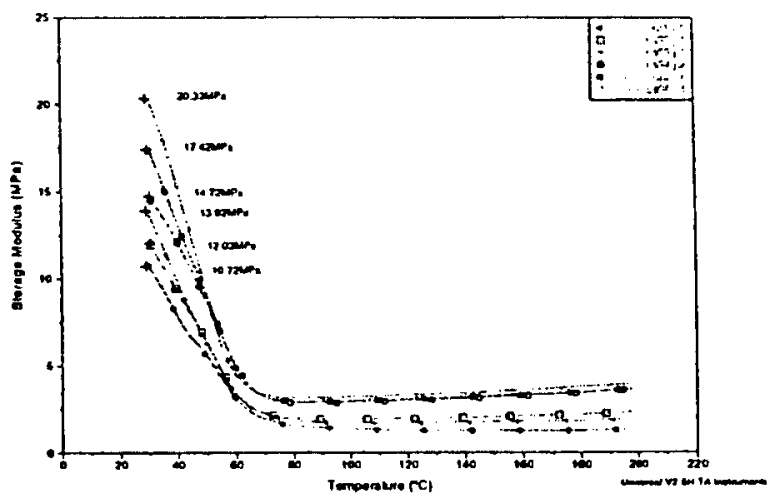

Figure 18. Storage modulus varied with percentage of type B epoxy diluent (DMA test)

Once we concluded that it is not possible to find a single material solution to replace our current bi-layer material, we focused on improving the DC1-2577, which is known to have poor resistance to jet fuel. We decided to chemically improve the siloxane base formulation (like DC Q1-4939) to generate desirable resistance to jet fume contamination and at same time preserve its intrinsic low stress mechanical property well below $-55^{\circ} \mathrm{C}$. Different catalysts were tried and concentration optimized. Addition of solvent-dissolved platinum catalyst reduced the peak curing temperature for the two-component hydrosilylation polymerization. Storage modulus measured with this 
formulation showed an excellent result even at low temperatures as shown in Figure 19.

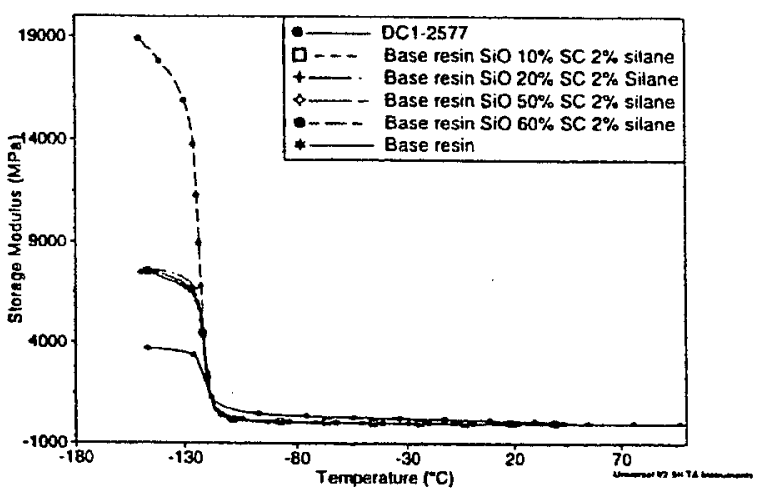

Figure 19 Storage modulus change with filler loading percentage for DC Q1-4939 series with the one for DC12577 as control. The weight percentage of filler in this series is $W_{\text {filler }} W_{\text {fuller }}+W_{\text {resin. }}$.

In order to increase the resistance to jet fuel, inorganic fillers were put into the base siloxane system to enhance its oleophobicity even though they typically inhibit the polymerization of the system and move the curing peak temperature a little higher. DMA tests were performed to verify that the storage modulus of the materials did not change too much. The Tgs of the series were maintained as low at $-120^{\circ} \mathrm{C}$ as that of the typical siloxane base system. The storage moduli of the series were lower then that of DCl -2577 at the temperature range of interest.

The chemical resistance tests were conducted on the base siloxane resins after curing at $150^{\circ} \mathrm{C}$ for $2 \mathrm{hrs}$ with or without filler loading and with or without silane adhesion promoter. Relative volume changes were measured after 1 and $5 \mathrm{~min}$. soaking in gasoline to accelerate its deterioration. Results are plotted in Figure 20. The results indicated that $10 \%$ filler loading can reduce the volume swelling by $14 \%$ for $1 \mathrm{~min}$ case. The addition of silane can further enhance the volume change resistance because of the slight cross-link density increase of the bulky siloxane.

The resistances of the resins cured at room temperature for $6 \mathrm{hrs}$ with and without filler loading or silane were also measured for their volume change. There was a significant difference among the samples with and without filler loading. Results as shown in Figure 21 indicate that the addition of filler reduced the swelling because of its improvement in oleophobicity. However, since silica itself is hydrophilic, overloading of the filler might inversely worsen the hydrophobicity of the system.

The calalyst used for accelerating the curing during this test was PC085 with $30 \mathrm{ppm}$ concentration. Even though there exists a tradeoff between chemical resistance and moisture uptake resistance, any potential degradation was considered to be minimal compared to improvement in jet fuel resistance.

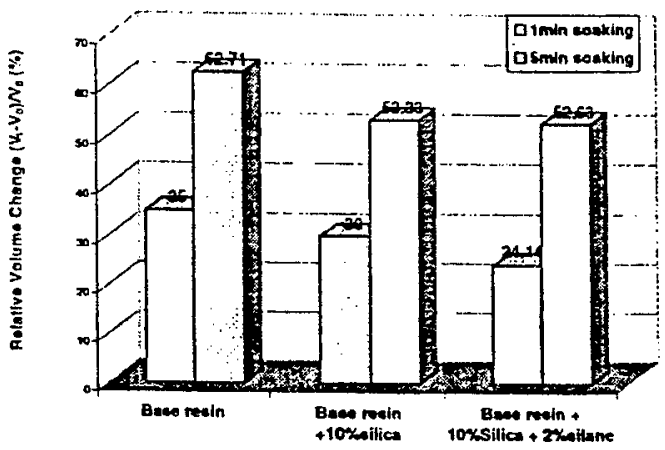

Figure 20 Material swelling vs. filler loading percentage of Q1-4939 base

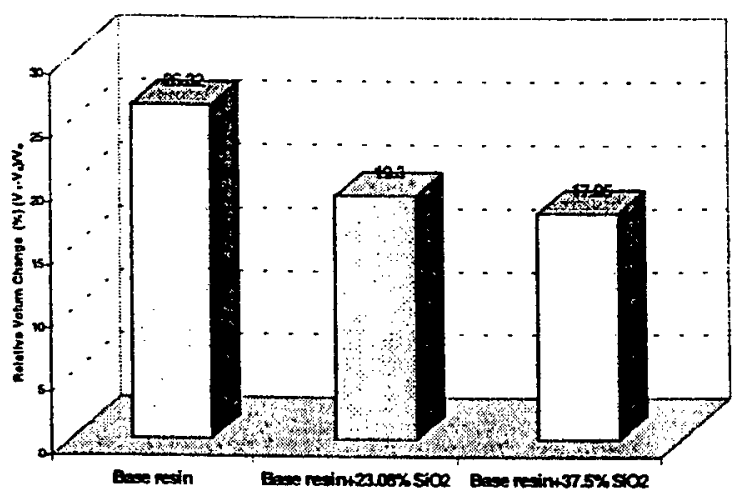

Figure 21 Material swelling vs. filler loading percentage of room temperature cured Q1 -4939 resin (after immersion in jet fume for $5 \mathrm{~min}$ ).

Storage moduli of the siloxanes before and after jet fuel exposure were also investigated. It was confirmed that the chemical structure and $T_{g} s$ of the siloxane did not change.

\section{CONCLUSIONS}

A MEMS-based sensor module and its application for as a 'pressure beit' for aircraft flight testing is described. It is an example of a multi-sensor system packaging configuration, which has been validated through two consecutive flight tests. Significant cost savings in aircraft flight load surveys are expected since it makes calibration and installation easier and simpler, and provides significantly better measurement accuracy and dynamic information. Reliability improvements have also been described. The newly developed low stress siloxane-based conformal coating has excellent resistance to jet fuel, has good fluidity, mechanical properties and extremely low $T_{z}$. 


\section{ACKNOWLEDGEMENTS}

This work was partly supported by DARPAAF_Research Laboratory under agreement F30602-97-2-0099. D.

Gilmour of Air Force Research Laboratory was the technical manager for the program. The authors would like to thank MEMS Pressure Belt IPT members at Boeing and Endevco for their continuous support. We also grcatly appreciate M. Chisa's support in module assembly. MCM substrates were fabricated by the Boeing Thin Film group. Contributions of Endevco personnel, R. Poff, J. Fragala and A. Karolys in the sensor area are also appreciated.

\section{REFERENCES}

1. N. P. Kim et al, "Smart Sensor Multi-Chip Module with Direct-Chip-Altached Micro-Electro-Mechanical
System (MEMS) Devices," Proc. Of $32^{\text {nd }}$ International Symposium on Microelectronics (IMAPS 99), p. 484 , 1999

2. J. Cech el al, "Reliability of Passivated Copper Multichip Module Structures Embedded in Polyimide," IEEE Trans. CHMT, v16, No 7, p.752, 1993

3. K. Coates et al, "Development of Thin Film Resistors for Use in Multichip Modules," Inter. Conf. On Multichip Modules and High Density Packaging, p. 490, 1998

4. N.P. Kim, K.L. Coates, G.G. Kunze, C.P. Chien, and M.H. Tanielian, "Development of Multi-Chip Modules with Integrated Thin Film Passive Elements," Proc. Of $30^{\text {th }}$ International Symp. Of Microelectronics (IMAPS 97), p. 157,1997 


\title{
Fabrication and Assembly of a Digital Transducer-to-Bus Interface Module Having a Directly Attached MEMS Device
}

\author{
Namsoo P. Kim, Nelli Amirgulyan, Kin Li, Chung-Ping Chien, and Minas H. Tanielian \\ The Boeing Company \\ Phantom Works \\ P.O. Box 3999, MS 3W-50 \\ Seattle, WA $98124-2499$ \\ (253) 657-5970, e-mail: namsoo.p.kim@boeing.com
}

\begin{abstract}
Boeing has developed a smart sensor system suitable for flight loads testing, which can accommodate large arrays of sensors in a bus configuration. In this paper, we report the development of a digital transducer-to-bus-interface module (TBIM) packaged as a multi-chip module (MCM). The most significant aspect of this module is the increased functionality at the sensor location. With local digital conversion, robust communication to the host computer is possible through a common bus eliminating the need for discrete wires. We also describe the fabrication and assembly processes used for this module, which contains a bare MEMS device, two ASICs (one analog and one digital), two memony chips and a number of discrete components. The MCM substrate was fabricated-on silicon using copper as conductor and polyimide as dielectric. The MEMS device was bonded using direct-chip-attachment process (flip chip) while other components were attached using conventional microelectronic interconnection and surface mount techniques.
\end{abstract}

Keywords: Micro-Electro-Mechanical Systems (MEMS), pressure sensor, direct chip attachment, flip chip, MCM

\section{Introduction}

Many different sensors are needed for aircraft and aerospace systems. They are used primarily to collect data, including static or dynamic pressure, shear stress, acceleration, velocity and noise. MicroElectro-Mechanical system (MEMS)-based sensors can provide significant advantages over conventional ones due to their small size. Even though MEMS are widely used in single sensor systems, implementation of sensors in array configuration is rare, mainly due to lack of suitable communication protocols. Boeing has been developing a MEMS multi-sensor system suitable for aerospace applications, including the hardware, firmware, and software necessary to run such a system. We have described earlier the development of an analog smart sensor module for flight load testing [1]. Its physical configuration and associated material choices were proven through multiple flight tests. In this paper, we report the development of a digital transducer-to-bus-interface module (TBIM) that can be directly incorporated into a common bus system. The most significant improvement over other efforts is the increased electrical functionality at the sensor location. With localized digital conversion, robust communication to the host computer is possible through a common bus eliminating the need for many wires used in analog systems.

\section{MEMS Based 'Pressure Belt'}

The technology currently used for aircraft flight testing is based on a plastic tube system with remotely located pressure sensors, discrete electronics, and pneumatic system control. It is shown in the lower portion of Figure 1. One of the drawbacks of this system is the pressure lag in the tubes. The tubes act as low-pass filters and since the distance between the hole drilled in the tube and the pressure scanner is different for each measurement the filter characteristics are different for each measurement. This limits the dynamic range of the system. As technology improves, it is desirable to have also increased sensor accuracy. Other drawbacks of this approach are the time it takes to install the entire system and the size and weight of the installation. The weight of the hardware for the current system could add 800 pounds on the wing. Furthermore, the installation is labor-intensive and thus costly.

The new MEMS based 'pressure belt' was developed to replace the current technology. The approach as 
shown in the upper portion of Figure 1 utilizes MEMS sensors located at the point-of-use, and high density MCMs containing associated electronic circuitry. The MCM accommodates functionality for localized data manipulation including temperature compensation and self-calibration. The MCMs connect to a flex circuit tape carrier, which carries the power buses and routes the data to the host computer. Figure 2 shows the overall system architecture of the pressure belt including the interface and the Network Capable Application Processor (NCAP) with the bus controller. Figure 3 and 4 show the physical schematic of the MEMS pressure belt. Figure 3 illustrates the various parts incorporated into the pressure belt while figure 4 shows a cross-section of the module packaging whose details are described in the later section of this paper.

\section{System Requirements and Module Design}

To be successfully implemented into any airborne hardware, the new system needed to meet various requirements. Two major parameters driving our system and module development were the thin profile and sensor accuracy. Other parameters taken into consideration were structural compatibility with target vehicle, operational temperature range, resistance to moisture and fuel vapor, and other general reliability constraints. The target accuracy of $0.1 \%$ of full scale on the system level is about an order of magnitude better than the typical accuracy possible with the current practices using the tube belt pressure measurement system. To meet this objective, module configuration and all the material systems were carefully chosen to minimize any stress being passed onto the sensor diaphragm. Stress can be transmitted from any underlying structure such as the airplane wing or even the MCM substrate itself. The choice of a Si MCM with as small a size as possible was so that it can be mounted on curved surfaces. The choice of $\mathrm{Si}$ as a substrate was to eliminate errors due to differential thermal expansion as this system has to operate from -60 to $80^{\circ} \mathrm{C}$. Finally, the thin profile of the module (approximately $0.070 \mathrm{in.}$ ) was needed for aerodynamic reasons.

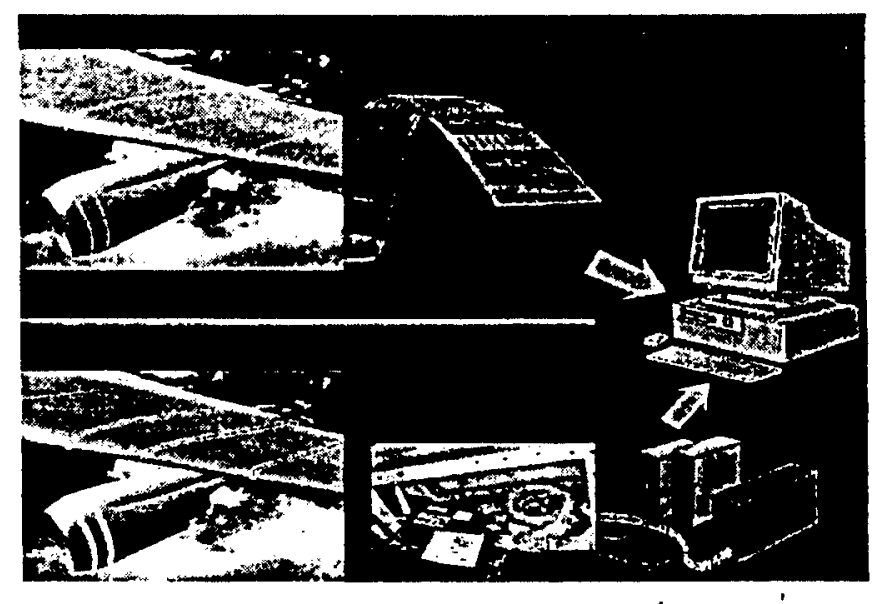

Figure 1. Aircraft flight testing (MEMS vs. Pneumatic Tube

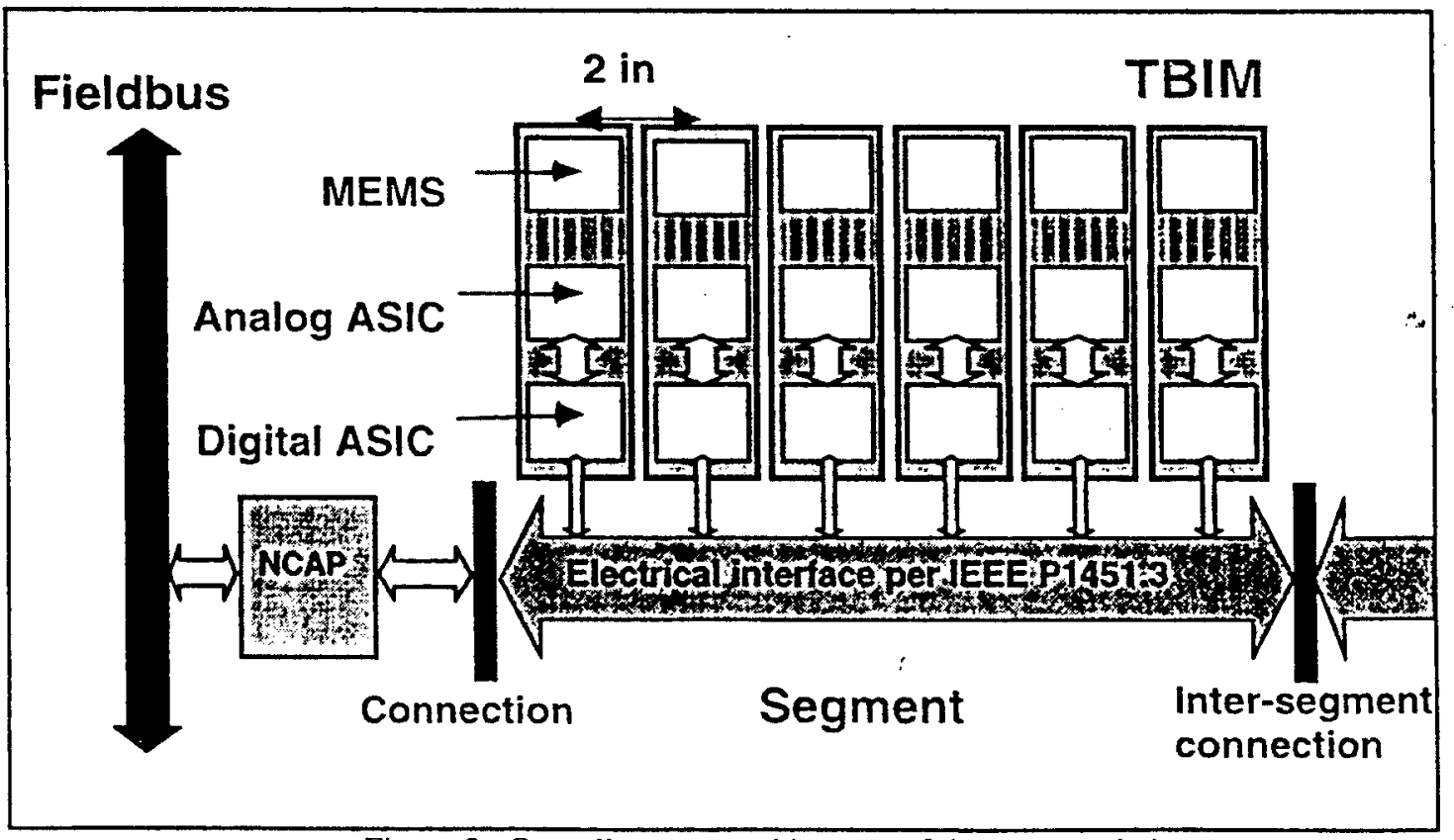

Figure 2. Overall system architecture of the pressure belt. 


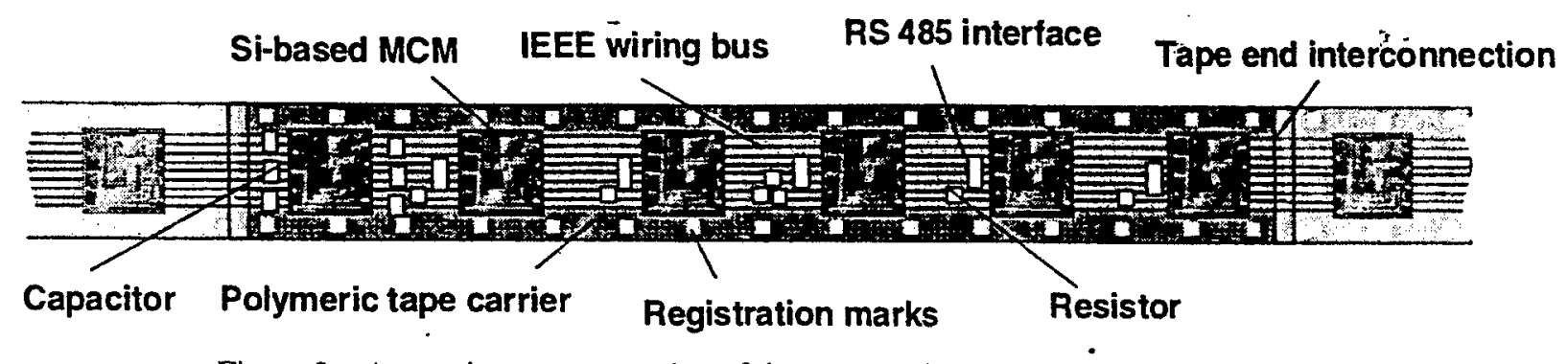

Figure 3. A top view representation of the pressure belt showing the various parts.

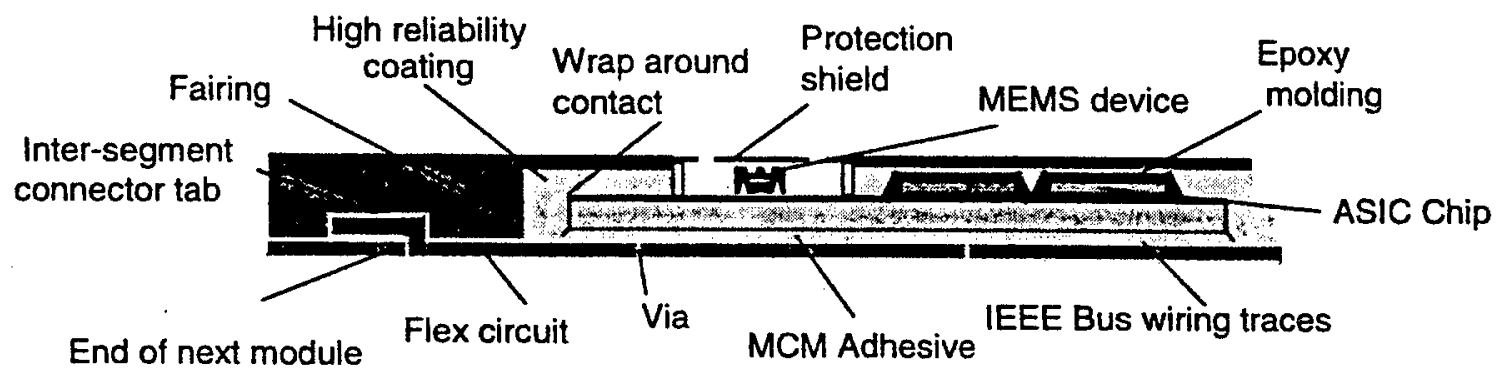

(Vertical scale enlarged for illustration only)

Figure 4. A schematic representation of the pressure belt cross-section and packaging.

Each TBIM MCM contains a MEMS pressure sensor, an analog ASIC, a digital ASIC, an EEPROM, a SRAM and 34 discrete components. The analog ASIC can perform signal conditioning for a number of different type of transducers such as piezoelectric, piezo-resistive, variable capacitance, and strain gages. The digital ASIC includes the core processor, digital filtering, a correction engine and a digital bus interface. The current design accommodates up to 127 TBIMs on a single bus based on 20 measurements/sec/sensor. The SRAM supports memory needed for data processing while the Transducer Electronic Data Sheet (TEDS.) is stored in the EEPROM and contains all the calibration data.

\section{MEMS Pressure Sensor}

The MEMS sensor that we used for this program was an ion-implanted silicon micromachined pressure sensor whose diaphragm was designed for 0-15 psia pressure range and remained the same as previously reported $[1,2,3]$. The rectangular sensor with .049 "x.067" dimensions incorporates a fully active Wheatstone bridge strain sensing circuit on the internal surface of the diaphragm. While the diaphragm currently in use is a standard part, the reference support for the vacuum chamber has been specifically designed to allow flip chip mounting of the MEMS assembly to the silicon MCM using solder bumps or conductive epoxy. This maintains the lowest possible profile since no wires extend above the top of the MEMS device which is only .016 inch thick. The special flip chip support provides feedthroughs so that electrical connections can be made from the backside of the sensor assembly. The feedthroughs consist of etched holes through the reference support. Pad metallization is then deposited which creates electrical bonding sites on the surface of the support and connects them to the pads on the surface of the diaphragm at the bottoms of the feedthroughs. The top and bottom sides of the MEMS sensor are shown in Figure 5 while Figure 6 illustrates improvements in detail.

\section{Substrate Design and Fabrication}

The 1.1" $\times 1.3 "$ MCM substrate was designed using copper as conductor and polyimide as dielectric using a standard Boeing process whose design guidelines have been reported elsewhere $[4,5]$. Figure 7 shows a schematic cross section of the module. It contains two main conductor and two via layers with additional metal layers for MEMS leads and surface pads. It was fabricated on a $10000 \AA$ oxidized silicon. 
The first metallization layer was a Titanium/Nickel/Gold stackup layer which forms the pads for direct chip attachment of the MEMS device, offering compatibility with solder and epoxy based flip-chip. Direct bonding to the silicon oxide surface eliminates any residual stress coming from the cured polyimide and also from thermal coefficient

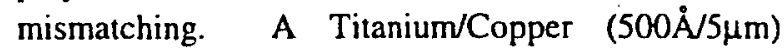
conductor layer was formed by electroplating $\mathrm{Cu}$ on the seed layer of sputtered thin film of Ti/Cu. Then the copper surface was patterned and wet etched. Another $500 \AA$ layer of titanium was sputtered, then the titanium was patterned and both layers of Ti dry etched in an RIE. The dielectric employed in this MCM was a pre-imidized photo-sensitive polyimide, HD Microsystem's PI2732. The polyimide was spin coated and baked for about 3 minute on hotplate bake at $100^{\circ} \mathrm{C}$. The polyimide was patterned in a projection aligner using broadband UV, then spray developed. A thermal cure in a vacuum oven followed by a plasma descum was performed. The second metal conductor stack was added in the same way for the first layer. Following this, the final pad layer including $\mathrm{Ni} / \mathrm{Au}$ was formed using a lift-off process. A layer of copper was then deposited, patterned and etched as hard mask to dry etch through the dielectric layer, exposing the MEMS pads which had been deposited in the beginning of the process. Removing the hard mask and cleaning completed the fabrication

process.

Figure 8 shows one unpopulated MCM unit with its original layout. Completed wafers were tested electrically and diced to produce good MCM units to be used for module assembly.

\section{Module Assembly and Belt Segment}

Module assembly was conducted by attaching various components to the MCM substrate. Various microelectronic assembly processes were employed. The MEMS device was attached to the MCM substrate using Ablestik conductive epoxy even though AuSn solder was also tried with good results. After the ASICs, and other ICs were die bonded, gold wire bonding was used for electrical connection due to their unavailability in flip chip form. Discrete components were attached using either conductive epoxy or solder reflow process.

In the opening through the polyimide layer a Pyrex glass tube wall was installed suriounding the MEMS sensor units. It is needed as a dam to the protective silicone coating applied later and to protect the MEMS sensor from any handling damage during the assembly and installation steps. Figure 9 shows a fully populated MCM unit. These fully populated
MCM units were tested for functionality using a test probe board. The functional test consists of applying power, establishing serial data communication, and verifying valid signal response from the module. Vacuum is applied to the MEMS sensors to see whether repeatable signal levels are observed within an acceptable range of the anticipated outputs. Dow Corning DC Q1-4939 (a very low stress gel) is applied between the MEMS device and the tube wall to protect the base region of the MEMS device and to prevent water invasion. Hysol Dexter 4460 (glob-top) epoxy is applied to protect the ICs completing the module assembly.

The flex circuit-based segment with a length of 12.46 inches containing $6 \mathrm{MCM}$ locations has been designed. A schematic of the segment design is shown in Figure 10. The flex circuit tape consists of two 0.0014-in thick copper layers on both sides of a 0.002 -in thick polyimide support with dielectric overlays on both sides.

\section{Flight Test Results and Discussion}

Various flight tests were performed to validate the physical design and packaging materials incorporated in the pressure belt module. The testbed used for these evaluations was the analog version of the pressure belt, which used the same materials in a similar form factor. The first airborne test was conducted using a 757-300 airplane undergoing flight tests prior to certification. Our pressure beli was attached in the belly section of the aircraft, close to the landing gear doors. The measured MEMS sensor readings fell well within the target of $0.1 \%$ of the full scale absolute pressure [2]. The second flight test was conducted with a 737 Boeing Business Jet (BBJ) conducting a mini-pressure survey to evaluate wing structural loads. Again the MEMS sensor data tracked well with the pressure data recorded from the flush reference pressure ports [2].

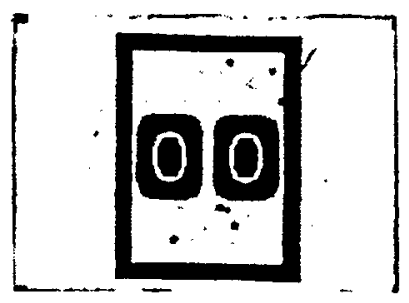

(a) Front side

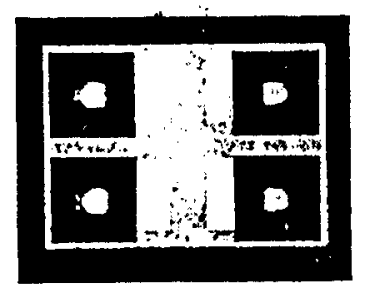

(b) Backside
Figure 5. Redesigned MEMS sensor 


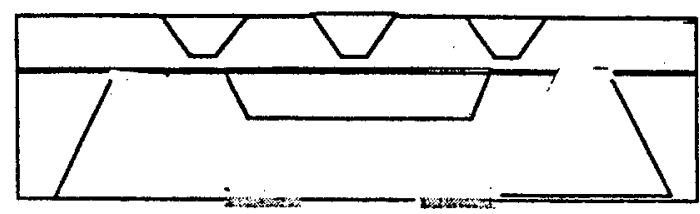

Figure 6. MEMS device feedthrough schematic

Another flight test was conducted using a F18-E aircraft. The pressure belt was attached to one of the airplane pylons because it provided easy access to the instrumentation bay. The reference sensor was located just below the sensor belt. The MEMS sensor test results are plotted in Figure 11 along with the reference pressure. A close comparison of the reference sensor reading and one of the MEMS sensor readings showed the difference in the dynamic behavior. As shown in Figure 12, the "peaks" and "valleys" are more filtered in the reference pressure readings.
Vibration tests were also carried out along 3-axis for 15 minutes each using the power spectrum shown in Figure 13. The calibration curve of the module was checked before and after the random vibration testing. Results are shown in Figure 14.

Reliability of our module configuration was improved by incorporating a protection shield on top of the Pyrex sensor tube wall. It was done to prevent a failure mode observed in the BBJ flight tests, when the airplane flew through a rainstorm. The improved MEMS unit is shown in Figure 15. We are currently fabricating the hardware needed for a full-scale validation of the technology. When fully implemented, we expect to reduce installation and hardware cost while offering an order of magnitude improved performance. Reduction of installation time will have added benefit for process cycle time reduction.

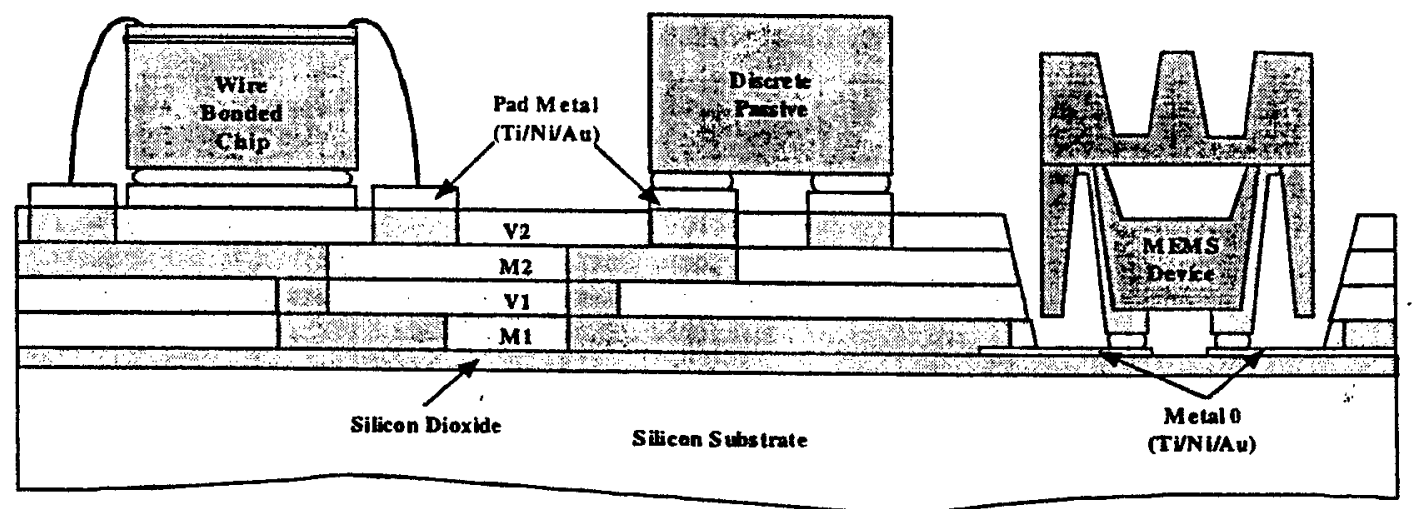

Figure 7. MCM cross section schematic
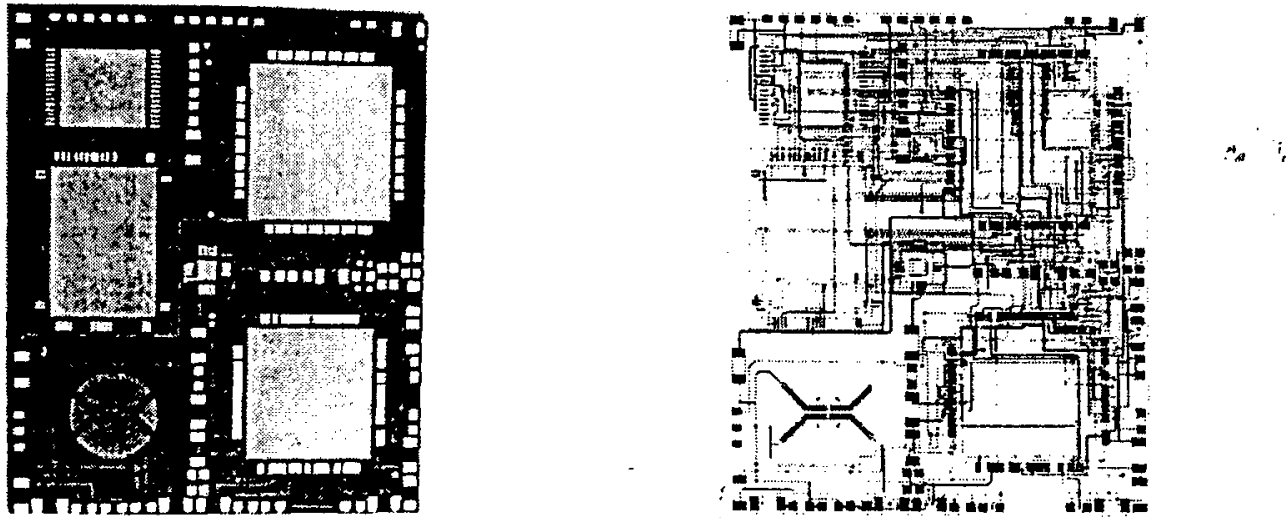

Figure 8. Fabricated MCM unpopulated unit with its layout 

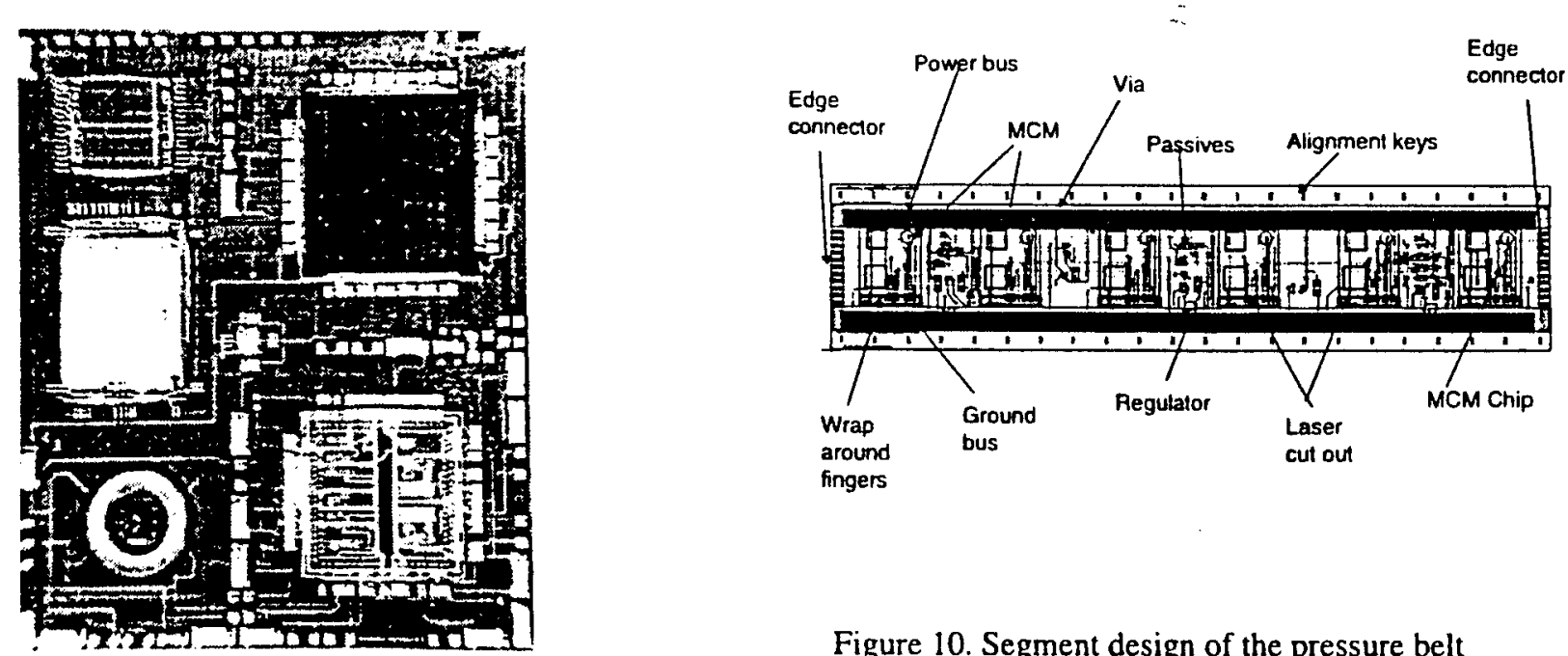

Figure 10. Segment design of the pressure belt

Figure 9. A fully populated MCM Unit

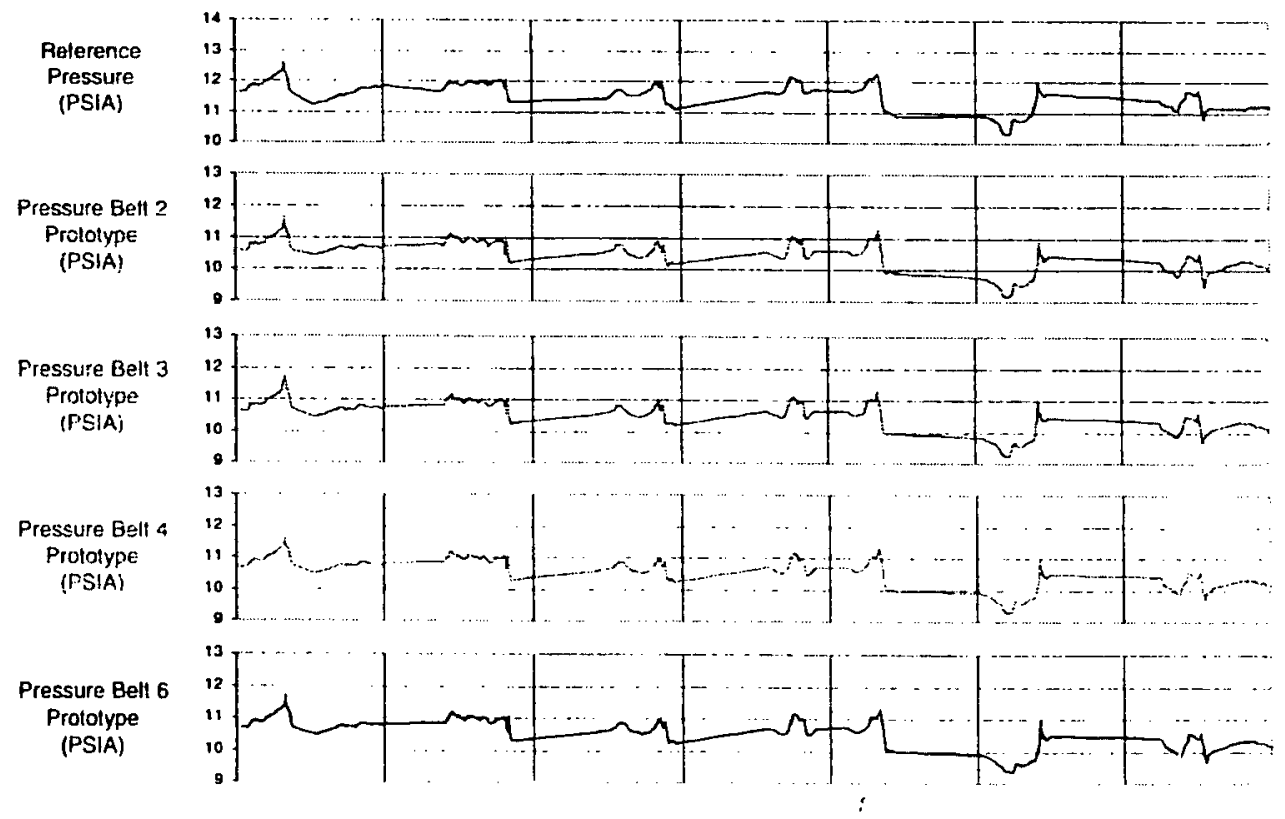

Figure 11. MEMS sensor results along with the reference data (top) 


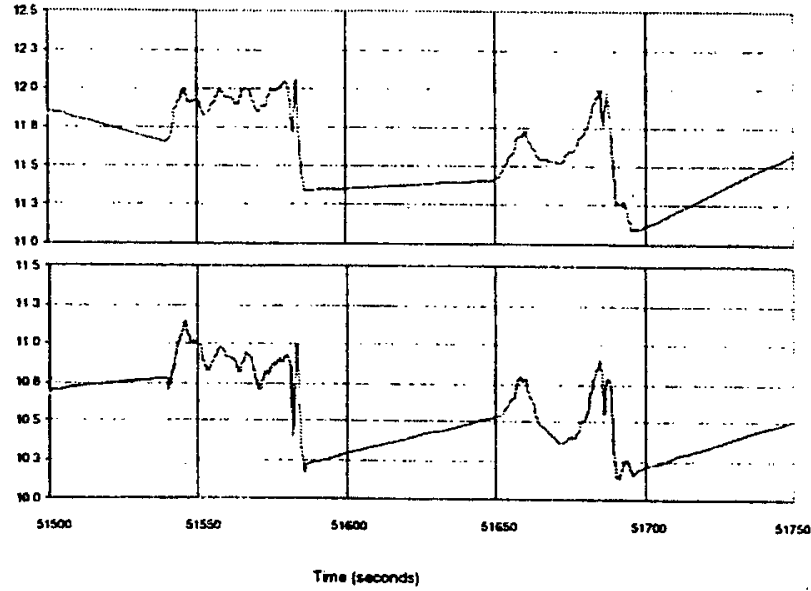

Figure 12. Close comparison between the reference sensor (top) and a MEMS sensor (bottom) data

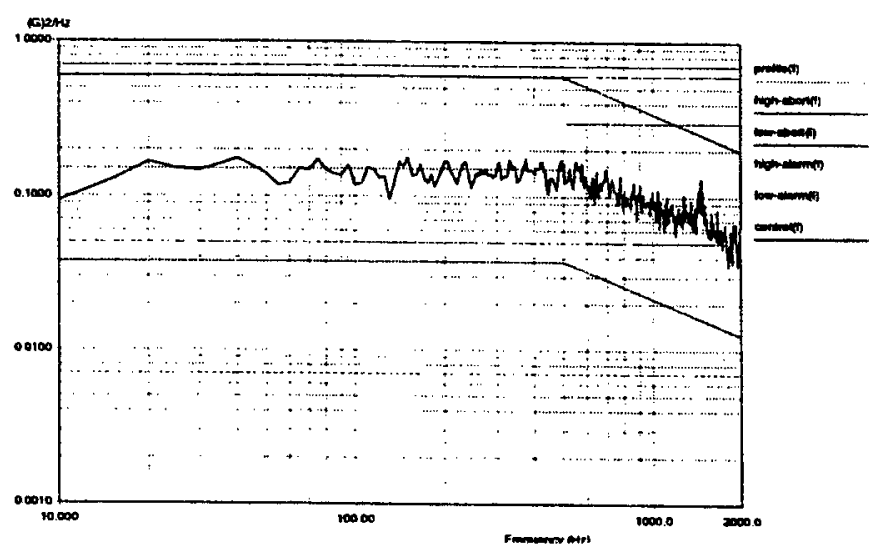

Figure 13. Random Vibration spectrum with $41 \mathrm{~g}$ peak values and an RMS value of $14-\mathrm{g}$.

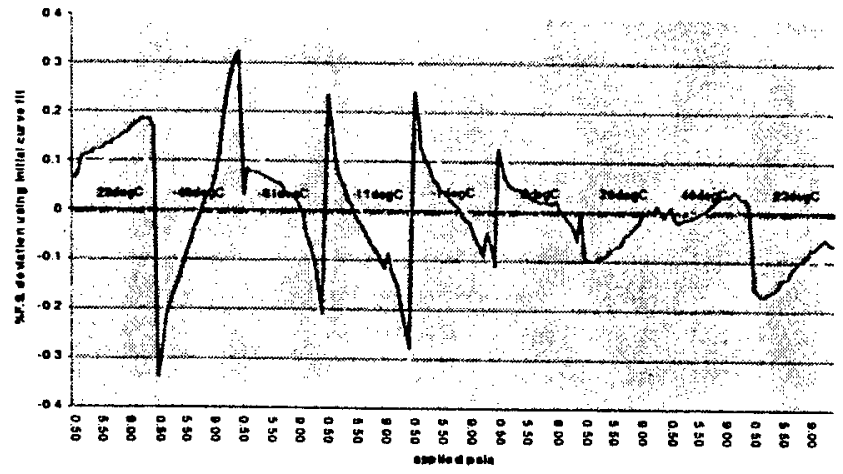

Figure 14. Sensor calibration curve before and after 3-axis vibration testing

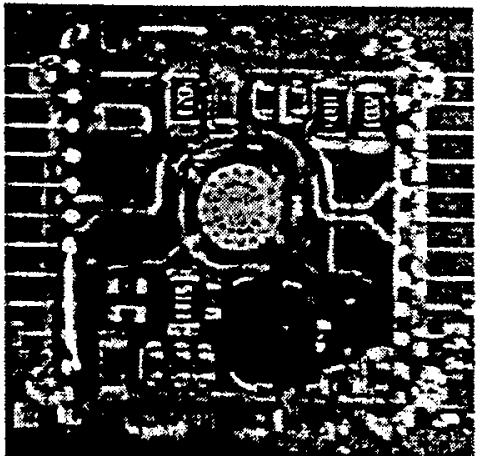

Figure 15. Module with protection shield

\section{Conclusion}

Development of a digital TBIM suitable for aircraft multi-sensor system applications is reported. Its mechanical configuration was confirmed through three flight tests. Reliability improvement is also described. With this new system, significant cost savings in our aircraft and aerospace products are expected because not only it is lower cost, but it also helps to make calibration and installation easier and more simplified.

\section{Acknowledgments}

This work was supported by DARPA/AFRL under agreement F30602-97-2-0099. The authors would like to thank Boeing MEMS Pressure Belt IPT members, especially $\mathrm{M}$. Holland for his module test support. Thanks are also extended to $M$. Chisa and $H$. Soares for module assembly, and J. Nielsen L. Branson, and C. Littlejohn for substrate fabrication. We also acknowledge Endevco's support in the sensor and electrical design area.

\section{References}

[1] N. P. Kim et al, IMAPS 99, p. 484, Oct. 1999

[2] N. P. Kim et al, Proc. Of 50th IEEE Electronic Components and Technology Conference, S18P4, May 2000

[3] N.P.Kim et al, Journal of Surface Mount Technology, v. 14, Issue 1, p. 1, Jan 2001

[4] J. Cech et al, IEEE Trans. CHMT, v16, No 7, p.752, 1993

[5] D. Kovach et al., IMAPS 99, p. 526, Oct. 1999 
APPENDIX B: PATENTS

"SYSTEM AND METHOD FOR MEASURING PHYSICAL PARAMETERS USING AN INTEGRATED MULTISENSOR SYSTEM"

Minas H. Tanielian, Namsoo Kim, and Mark Holland.

U.S. Patent \# 6,134,485

Oct. 17,2000 


\section{United States Patent}

[54] SYSTEM AND METHOD FOR MEASURING PHYSICAL PARAMETERS USING AN INTEGRATED MULTISENSOR SYSTEM

[75] lnventors: Minas H. Tanielian; Narnsoo Kim, both of Bellevue; Mark J. Holland, Port Orchard, all of Wash.

[73] Assignee: The Boeing Company, Seattle, Wash.

[21] Appl. No.: 09/252,584

[22] Filed: Feb. 18, 1999

[51] Int. Cl.? B64C 11/34

[52] U.S. Cl. 701/14; 701/1;701/2. $244 / 17.13 ; 244 / 200 ; 244 / 203 ; 73 / 178 \mathrm{~T}$ $340 / 945 ; 340 / 973$

[58] Field of Search $701 / 14,1,2,7$ $701 / 8,9 ; 244 / 17.13,200,203 ; 73 / 178 \mathrm{~T}$; $340 / 945,973$

\section{References Cited}

\section{U.S. PATENT DOCUMENTS}
$5,623,411$
4/1997 Morvan $701 / 14$ $701 / 14$

5,986,580 11/1999 Permanne $340 / 946$

Primary Examiner-William A. Cuchlinski, $\mathrm{J}$. Assistant Examiner-Yonel Beaulieu Attorney, Agent, or Firm-Brooks \& Kushman P.C.

\section{ABSTRACT}

A system and method for analyzing physical parameters of flight data at a plurality of discrete locations about a surface of an aircraft includes a multisensor system having an array of belts. Each belt includes a phrality of interconnected belt segments including a substrate baving an electrically conductive digital data bus, and at least one module having a first sensor, a second sensor and a digital signal processor, and a coating for protecting the belt segment. The first and second sensors, which are preferably formed as microelectromechanical sensors sharing a common substrate, respectively generate signals representative of a first physical parameter and a second physical parameter. The processor receives and analyzes the first and second signals to generate a third signal. The third sigual is transmitted along the electrically-conductive bus to a remotely-located controller. The controller analyzes the third signal to obtain flight status information relating to the effect of the physical parameters on the flight.

29 Claims, 3 Drawing Sheets

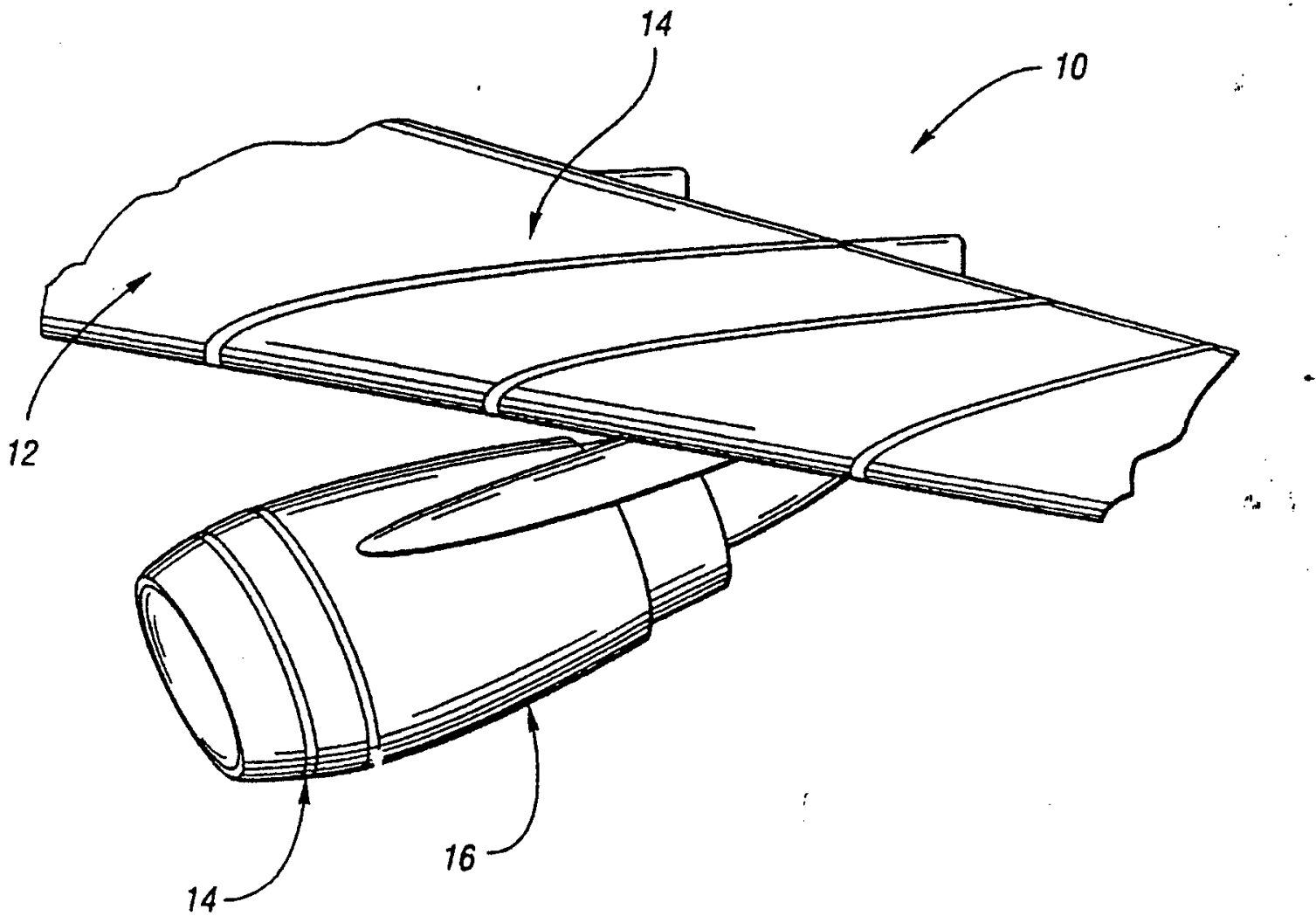




\section{U.S. Patent Oct. 17, $2000 \quad$ Sheet 1 of $3 \quad \mathbf{6 , 1 3 4 , 4 8 5}$}
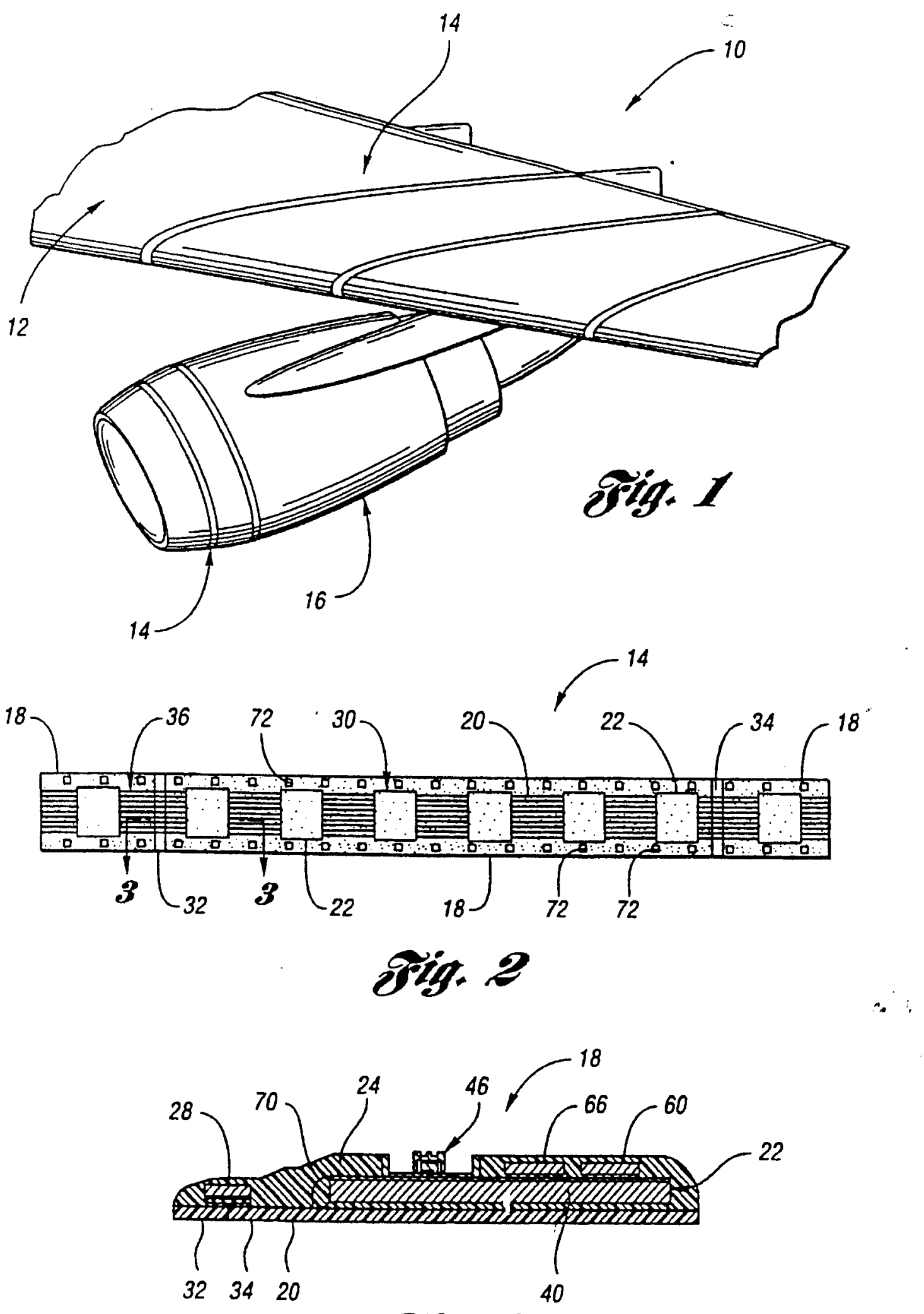

Jig. 3 


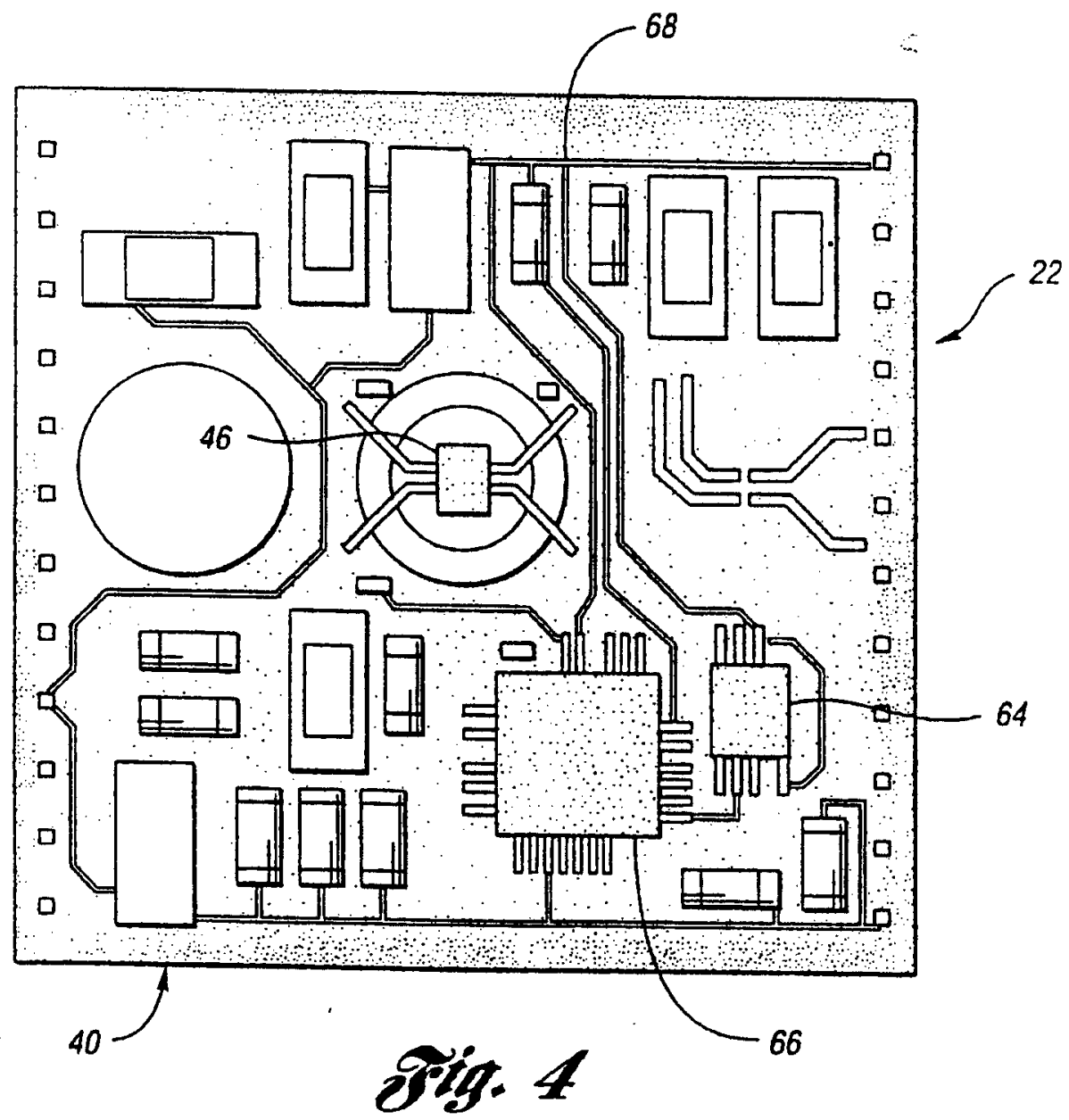

ชัभुg 5

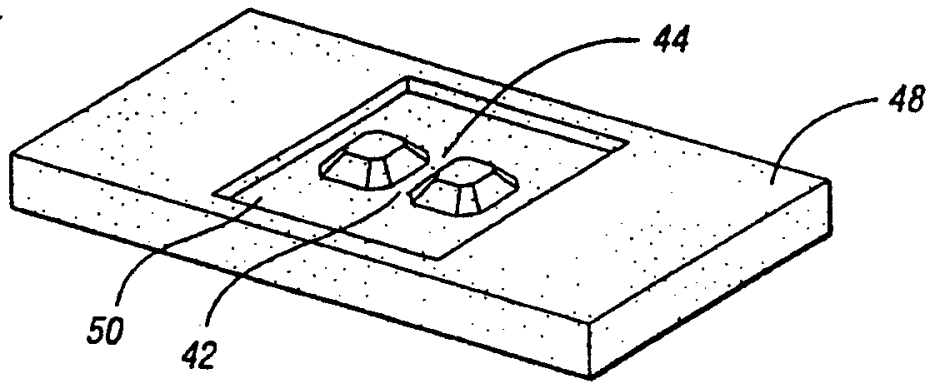

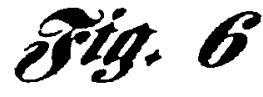

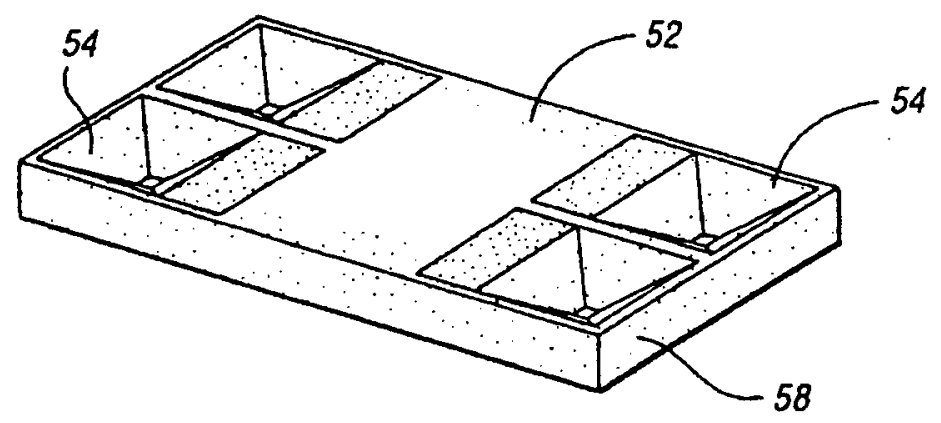



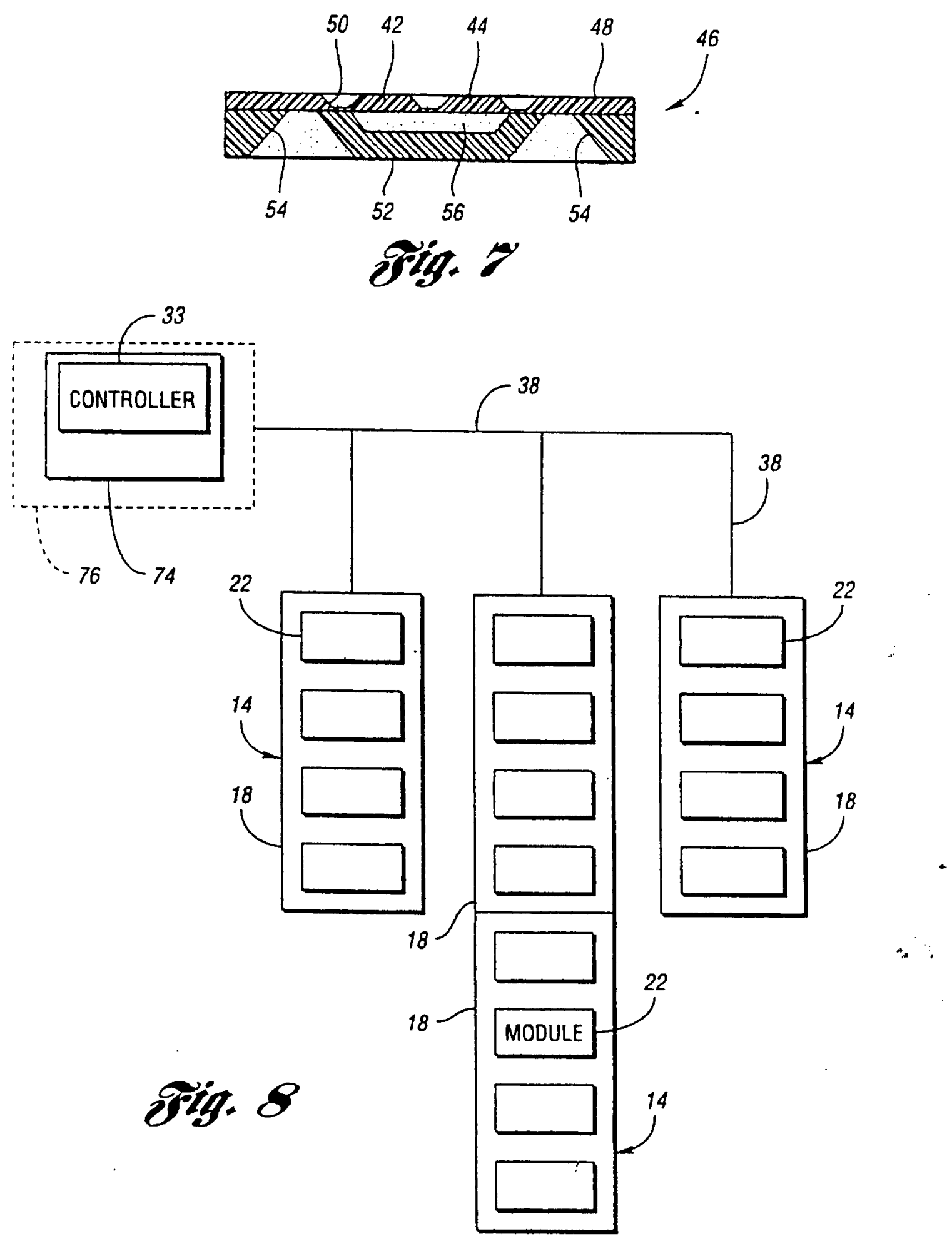


\section{1 \\ SYSTEM AND METHOD FOR MEASURDNG PHYSICAL PARAMETERS USING AN INTEGRATED MULTISENSOR SYSTEM}

\section{FIELD OF THE INVENTION}

This invention relates to a system and method for measuring and analyzing physical parameters about a surface of an object using an integrated multisensor system.

\section{BACKGROUND INFORMATION}

The prior art has generally recognized the need for measuring and analyzing physical parameters achieved at discreet locations about the surface of an object, often in the context of an object moving through a particular fluid. Examples include measuring the physical parameters achieved about the surface of a boat bull as it moves through water, or aboul the airframe of an aircraft during flight. Another example includes measurement of stress and strain achieved at discreet locations about a load-bearing structure.

By way of example, aircraft pilots and designers require information detailing the effects of physical conditions on an aircraft during flight. Designers use testing devices placed about an airframe of an aircraft to create flight loads surveys, which measure physical parameters across various parts of the airframe during flight. A typical flight load testing system monitors physical parameters about the body, wings, tail, nacelle and engine of an aireraft. Such fight load testing systems are typically either removably affixed to an aircraft or are integrally mounted into the airframe of an aircraft for use in regular flighl operations.

A typical flight load testing system includes a series of pressure sensors disposed within paeumatically-controlled plastic tubes that are removably affixed to the eircrafi. The tubes are placed about the airframe of an aircraft at various positions to gather sensor data relating to the physical parameters affecting the airplane during flight. Leads extend from each tube to a remote data processing device having signal conditioning electronics. Such removable lesting systems are typically cumbersome to install and are expensive to operate and maintain. Additionally, the testing systems increase the weight and drag along the airframe of the aircraft, which results in a lack of accuracy in determining physical parameter data during the flight tests.

An example of a type of physical parameter lesting system is disclosed in U.S. Pat. No. 5,001,638 to Zimmerman et al. The Zimmerman ' 638 patent discloses an integraled aircraft air data system which includes first sensors mounted to the airframe for measuring pressure along the airframe, and second sensors mounted to the airframe measuring lotal temperature. The first and second sensors are typically mounted at separate locations and sead analog outputs along a bidirectional data bus to a centrally-located air data unit. The analog outputs are converted to digital signais at the centrally-located air data unit. The air data unit then provides air pressure and temperature data for the aire aft's cockpit instrumentation and for controlling the airctaft's engines.

\section{SUMMARY OF INVENTION}

A system and method for measuring pbysical paramelers at a plurality of discrete locations about a surface of an object is provided. The system comprises a multisensor system including at least one belt segment having a plurality of sensors and processors in communication with a remotely-located controller along an electrically-
2

conductive, bidirectional digital data transfer bus. For example, where the system is applied to colleet aircraft flight data, a plurality of interconnected belt segments are disposed about the body, wings, nacelle, tail and engine of the 5 aircraft to provide measurements of various physical parameters during a flight loads survey of an aircraft configuration. The multisensor system may be used in a variety of different applications, including measuring physical parameters about the rotors of a belicopter, about the bull of a boat or 10 submarine, and about the body of an automobile.

In accordance with the invention, each belt segment includes a polymeric tape carrier having integrally-formed wire traces defining a portion of the system's electricallyconductive bidirectional digital data bus; two or more

15 parameter-sensing modules affixed to the tape carrier in electrical communication with the bidirectional digital data bus; and a coating for protecting the tape carrier and the modules from environmental conditions, as well as to provide the belt segment with improved aerodynamic proper-

20 ties. Where the physical parameters are to be measured as the object moves through a given fluid, the tape carrier bas a generally planar surface and a nominal thickness that is significantly less than the boundary separation layer defined upon movement of the object through the fluid. In this 25 manner, the belt segment is provided with a reduced acrodynamic profile to allow for more accurate physical parameter measurements.

In the event that two or more belt segments are employed, each belt segment has end portions adapted for interconnection with an adjacent belt segment. An interconnection device, such as a low-profile connector, physically and electrically interconnects adjacent belt segments to connect the module-mounted sensors/processors of each belt segment with a remotely-located controller, such as a networkcapable application processor (NCAP).

A combination of one or more protective coatings encapsulates at least a portion of each belt segment and module. The coatings not only protect the belt segment from adverse environmental conditions, but also define an aerodynamic surface on each belt segment. By way of example only, the coating may either be comprised entirely of one compound or may be a multilayer combination of organic or inorganic compounds, preferably a bi-layer or tri-jayer combination, suilably engineered to provide the best combination of environmentally protective and aerodynamic properties.

Each of the modules affixed to the tape carrier includes. a pair of sensors, each sensor generating an analog signal. representative of its respective physical parameter; an arta-

so log integrated circuit (IC) to provide signal conditioning for the analog signal generated by each sensor; a memory IC; an analog-to-digital converter; and a digital signal processor. Interconnection leads provide electrical communication between the digital signal processor of each module to the 55 data bus defined in the tape carriers, thereby providing communication pathways between the modules and the NCAP.

More specifically, the first sensor atlached to each module measures a predetermined physical parameter and generates 60 a signal representative of the physical parameters measured. The second sensor is attached to the module adjacent to the location of the first sensor. The second sensor also measures a predetermined physical parameter and generates a sigoal representative of the physical parameter measured. By way s of example only, the physical parameters to be measured by the first sensor may be pressure and temperature by the 147 
signals based on the physical parameter measurements, which are tben processed and amplified by an analog integrated circuit (IC), and are then converted to digital signals. The digital signal processor uses the signal from the second sensor to effect correction on the signals generated by the 5 first sensor.

Additional physical parameters measured by the sensors may include, without limitation, static pressure, absolute temperature, acceleration, chemical environment, mechanical strain and component aging. In a preferred embodiment of the invention, the first and second sensors are formed on a common substrate, which is bonded to the module sub. strate. Alternatively, the sensors may be formed by a multiplicity of processes which otherwise result in a given pair of first and second sensors being allached to the same 15 module. The modules are then attached in an array, preferably a one-dimensional array, along the surface of the tape carrier.

A digital signal processor is affixed to each module proximate to the location of the first sensor. The processor receives and analyzes the physical parameter signals respectively generated by the module's pair of first and second sensors. The processor itself is in electrical communication with the bidirectional digital data bus through electrical interconnection paths, such as tape automated bonds (TAB), whereby the processor transmits both the processor node address and the third processed digital signal along the tape carrier's integrated bidirectional data bus to the network capable application processor (NCAP).

A remotely-located controller, normally disposed within the airframe of an aircraft, is electrically connected to the bidirectional digital data buses of the one or more belt segments. In a preferred embodiment, the controller is part of the network-capable application processing device (NCAP). The controller communicates with exch belt segment's modules to collect the third signal generated by each digital signal processor. The controller analyzes the collected third signals based on a predetermined set of instructions. The controller communicates with the modules using a standard protocol over the system's shared electricallyconductive digital data buses.

In a preferred embodiment, the controller assesses the integrity of the third signals generated by the digital signal processor of each module, as well as sending commands to the module about when a measurement should be taken and verifying the health status of the plurality of modules. Alternatively, the controller can forward the data to the instruments on the aircraft to provide flight status information during operation of the aircraft. Additionally, the controller may store the physical parameter data collected during aircraft flight, possibly to be downloaded by aircraft maintenance support personnel afier completion of the flight. The controller may also transmit the physical parameter data via a wireless interface to the ground.

\section{BRIEF DESCRIPTION OF DRAWINGS}

FIG. 1 is a perspective view of a portion of the aiffame of an aircraft, wherein an amay of belt segments of an exemplary multisensor system are attached to the aircraft's wing and nacelle.

FIG. 2 is a top view of a portion of the array of belt segments of the exemplary system;

F1G. 3 is a transverse cross-sectional view along line $3-3$ of FIG. 2;

FIG. 4 is a top view of a parameter-sensing module prior to attachment to a belt segment;
FlG. 5 is a lop perspective view of a microelectromechanical sensor of the exemplary sysiem;

FIG. 6 is a bottom perspective view of the microelectromechanical sensor shown in FG. 5 ;

FIG. 7 is a cross-sectional view of the microelectromechanical sensor shown in FIGS. 5 and 6; and

FIG. 8 is a diagrammatic schematic of the exemplary system illustrating the communication pathways of the electrically-conductive data bus includiog the interface module between the array of bell segments and the remotely-localed controller.

\section{DETAILED DESCRIPTION OF THE INVENTION}

Referring to FIG. 1, a multisensor system 10 for measuring physical parameters achieved at discrete locations about the surface of an eircraft 12 during flight includes an amay of interconnected belts 14. As seen in FIG. 1, the array of interconnected belis 14 is mounted to the aircraft's airframe, such as the aircraft's wing and pacelle of the aircraft 12. It will be appreciated that other suitable locations for deploying the belis 14 to collect aircraft flight data include select surfaces on the body, wings, nacelle, tail and engine of the aircraft 16. And, in the exemplary system 10 illustrated in the drawings, the belts 14 are disposed about the surface of the airframe so as to define a two-dimensional array along the airframe to allow for physical parameter measurements at multiple locations about a common surface.

Eacb belt 14 includes at least one sensor-carrying belt segment 18. More specifically, as seen in the exemplary system 10 illustrated in FIGS. 2 and 3, each belt segment 18 includes a relatively-flexible substrate, such as a thin polymeric tape carrier 20; at least two parameter-sensing mod35 ules 22 affixed to the tape carrier 20 , and a protective coating 24 covering at least a portion of both the tape carrier 20 and the modules 22.

More specifically, in the exemplary system 10, the tape carrier 20 forming the substrate of each belt segment 18 has to aominal thickness such that the total thickness of the belt segment is significantly less then the thickness of the boundary separation layer defined about the aircraft during flight. The reduced nominal tape carrier thickoess creates a relatively acrodynamic profile for the belt segment 18 , thereby as allowing the modules 22 on each belt segment 18 to take more accurate physical parameter measurements, while the tape carrier material is selected to allow the belt segment 18 to conform to non-planar surfaces of the airframe. The tape carrier material is also preferably selected to otherwise so reduce the weight of each belt segment 18.

Thus, by way of example only, in a constructed embodiment of the exemplary system 10 , wherein eacb beli segment 18 is about $305 \mathrm{~mm}$ (12 inches) in length, the tape carrier 20 is preferably formed of a polymeric material having a ss thickness in the range of $0.025 \mathrm{~mm}$ to $0.25 \mathrm{~mm}(0.001$ inches to 0.01 inches). Up to six parameter-sensing modules 22 are mounted on each $\mathbf{3 0 5} \mathrm{mm}$ belt segment $\mathbf{1 8}$.

A series of wiring traces 30 are integrally formed into the tape carrier 20 of each belt segment 18. An interconnection 60 device, such as a low-profile connector 28 , is used to pbysically and electrically interconnect the end portions 32,34 of adjacent belt segments 18. Together, the electrically interconoected wiring traces 30 of the belt segments 18 define a bidirectional data transfer bus, indicated generally 65 at 36, which exlends along the entire length of each bell 12. The bus 36 allows both digital and analog signals 10 pass 148 
modules 22 and a remolely-located controller 38 , to be described in greater detail below.

As seen in FIGS. 2-4, the modules 22 attached to each belt segment 18 each include a module substrate $\mathbf{4 0}$ formed, for example, of oxidized silicon. A pair of analog-output physical-parameler-data sensors $\mathbf{4 2 , 4 4}$ are centrally mounted in close proximity on top of each module substrate 40. While the invention contemplates use of any suitable sensors, in a constructed embodiment of the exemplary system 10, the sensors 42,44 are piezoresistive seasors.

Referring to FIGS. 5-7, in the exemplary system 10, the first and second seasors 42,44 are absolute-reference micromachined silicon sensors advantageously formed on a common substrate to form a microelectromechanical system (MEMS), indicated generally at 46 . The MEMS 46 is itself attached to the module substrate using a conductive epoxy. Moreover, in the exemplary system 10, the MEMS 46 is designed to have a low profile to increase the aerodynamic efficiency and accuracy of the physical parameter measurement process. Thus, in a constructed embodiment, MEMS 46 has a profile of less than $1 \mathrm{~mm}$. The first sensor 42 is part of a micromachined pressure diaphragm capable of genetating a first analog signal representative of absolute static pressure up to perhaps about $103.5 \mathrm{kPa}$ (about $15 \mathrm{psia}$ ). The second sensor 44 generates a second analog signal repre. sentative of absolute temperature in a range of about $-50^{\circ} \mathrm{C}$. to about $85^{\circ} \mathrm{C}$.

It will be appreciated, however, that, depending upon the application to which the multisensor system of the invention is being put the first and second sensors 42,44 may generate analog signals representative of other sensed physical parameters, including, without limitation, acceleration, chemical environment, mechanical stress, mechanical strain and component aging. In this regard, each module 22 may also advantageously include additional sensor mounting locations 46 on either side of the center-mounted sensors 42,44. Additional sensors may thus be mounled on either side of the first and second sensors $\mathbf{4 2 , 4 4}$ for measuring additional physical parameters, including evaluating the differences in the first and second sensor's stress sensitivity. Where desired, the invention contemplates use of suitable mechanisms for mechanically isolating a given sensor from the tape carrier 20 to improve sensor performance.

Returning 10 FIGS. 5-7, in the exemplary system 10, the MEMS 46 includes a top surface 48 baving a recessed area which forms a diaphragm 50 containing the first and second sensors 42,44, and a bottom portion 52 baving a plurality of recesses 54 . The second sensor 44 is located proximate to the first sensor $\mathbf{4 2}$ on the back surface of diaphragm $\mathbf{5 0}$ inside cavity 56 in order to preserve sensor reliability/measuring accuracy.

The bollom portion 52 of the MEMS 46 includes a cavity 56 defined within a reference suppor 58 . The reference support 58 is designed to allow tlip chip bonding of the MEMS 46 to the module substrate 40 of the module 22 . The MEMS 46 is mounted to the module 22 using solder bumps or conductive epoxy. The use of flip chip bonding keeps the profile of the module-mounted MEMS 46 to about $0.41 \mathrm{~mm}$ ( 0.016 inches) thick. The reference suppor 58 advantageously provides feedthroughs so that electrical connections can be made from the backside of the MEMS 46. The feedthroughs consist of etched boles through the reference support $\mathbf{5 8}$. A suitable barrier metal, such as gold, is deposited through the etched holes, crealing electrical pads on the surface of the reference support 58 to conoect them to the pads of the surface micromachined sensors 42,44 .
The MEMS 46 incorporates a fully-active Wheatstone bridge strain sensing circuit on the internal surface of the diaphragm 50. The vacuum reference is accomplished with a second reference support 58 attached to the back of the 5 diapbragm 50 . The second reference support is bermelically glass sealed to the sensor and has a cavity which is evacuated during the sealing process. The cavity always presents the stable reference vacuum.to the back side of the diaphragm 50.

10 Returning to FIGS. 2-4, each module 22 also includes an analog signal-conditioning integraled circuit (IC), for example, an analog signal-conditioning processor 60 optimized for piezoresistive sensors. In a constructed embodiment, the analog signal conditioning processor 60 is 5 bonded to the module substrate 40 using a non-conductive epoxy. It will be appreciated, however, that the analog signal-conditioning processor 60 may otherwise provide suitable signal conditioning for other lypes of transducers employed on the module 22, including piezoelectric, 20 piezoresistive, variable capacilance, variable charge and strain gages.

Each module 22 furtber includes a memory IC 64, an analog-to-digital converter, a digital signal processor 66, and associated circuitry, indicated generally at 68 , for electrically interconnecting the first and second sensors $\mathbf{4 2 , 4 4}$ with the other module components $60,64,66$. The digital signal processor 66 communicates with the bidirectional data bus 36 defined in the tape carrier 20 , and power is provided to module components, via suitable electrical interconnection paths 70. By way of example only, in the exemplary system 10, the electrical interconnection path 70 is provided by tape automated bonds (TAB).

The digital signal processor 66 additionally contains a digital filter, a correction engine and a microcontroller to provide basic operational functions for the module 22. Each digital signal processor 66 has a specific node address which identifies the processor to the remotely-located controller 38. The digital signal processor 66 collects and analyzes the ${ }_{40}$ first and second analog signals which have been respectively generated by the first and second sensors 42,44 , amplified by the analog signal-conditioning processor 60 , and converted to digital signal by the analog-to-digital converter. The digital signal processor 66 generates a third, digital signal, 5 based on the conditioned/amplified and digitally-convented frst and second analog signals, which is thereafter transmitted to the controller 38 in response to a call signal, as described more fully below. In accordance with one feature of the invention, the digital form of the third signal ensuites that signal inlegrity is maintained during its transmission along the data bus 36 to the controller 38 .

A series of registration marks 72 are preferably provided on the tape carrier $\mathbf{2 0}$ to facilitate attachment of the modules 22 to the tape carrier 20 at specified discrete locations 35 thereon, whereby the module-mounted sensors 42,44 define a one-dimensional sensing array along the length of the belt segment 18. A two-dimensional sensing array may thus be achiev ad by using multiple belts 14 , each of which includes at least one physical-parameter-data-sensing belt segment 60 18, as illustrated in FIG. 1. Altematively, the invention contemplates placement of the modules 22 on each belt segment 18 such that the module-mounted sensors 42,44 themselves define a two-dimensional sensing amay.

Referring again to FIGS. 2 and 4, as noted above, a 65 protective coating 24 encapsulates a portion of each interconnected belt segment 18 . The coating 24 protects the belt 149 
aircraft flight and otherwise provides the belts 14 with an aerodynamic surface. In the exemplary system 10, constructed embodiment, the coating 24 covers the entire belt segment 18 except for the recessed area 50 of the top surface 48 of the MEMS $\mathbf{4 6}$. The uncoated recessed area 50 of the MEMS 46 allows the first and second sensors 42,44 to directly measure their respective physical parameters.

The coating 24 may be formed from a single compound, or it may comprise a multilayer combination of organic or inorganic compounds, preferably a bi-layer or tri-layer combination, suitably engineered to provide the best combination of environmentally proteclive and aerodynamic properties. For example, in a constructed embodiment, a tri-layer coating is employed: the first layer, designed to provide a bumidity barrier for the module's circuitry 68 , is formed of a coating material such as Hysol Dexter FP4460 epoxy. The second layer, desigaed to provide an aerodynamically-smooth surface profile, is formed of a conformal coating material such as Dow Corning DC 3-1753. The third layer, designed to prevent water invasion 20 at the base region of the MEMS 46, is formed of a protective material such as Dow Coming DC Q1-4939.

A diagrammatic schematic overview of the exemplary system 10 is illustrated in FIG. 8. In FIG. 8, the array of interconnected belis 14, themselves disposed about the surface of the aircraft 12 to thereby place the parametersensing modules 22 of each constituent belt segment 18 at a plurality of discrete locations about the surface of the aiffame (not shown). The bidirectional data bus 36 defined by the interconnected belts 14 is itself connected to a remotely-located controller 38 . In a constructed embodiment of the exemplary system 10, the controller 38 is part of a network capable application processor (NCAP), indicated generally at 74, which can be connected to a communication network used to interface to a bost computer, indicated generally at 76. In an alternative embodiment, the controller 38 can be configured as a host computer to interface directly with the communication network without the use of the network capable application processor.

As noted above, the diginal signal processor 66 of each segment-mounted module 22 communicates with the remotely-localed controller 38 across the bidirectional data bus 36 integrally-formed within each belt's tape carrier 20 . The data bus 36 includes a high speed bus and a low speed bus for communication belween each module's digital signal processor 66 and the controller 38. The high speed data bus is used for time-critical operations such as data transfers between each digital signal processor 66 and the controller 38, sample synchronization and memory download/selfidentification protocols. The low speed data bus is used for non-time critical operations, such as command and status operations, as well as support of the self-identification protocol.

In the exemplary system 10 , the third, digital signals generated by each digital signal processor 66 are transferred along the data bus 36 to the controller 38 using a sman transducer serics protocol such as IEEE P1451 or equivalent. The controller 38 performs bardware address filtering for all signal packets and hardware command decoding for specific commands. This allows the controller 38 to provide deterministic time response to specific commands, such as the trigger command, to collect signals transmitted from each module's digital signal processor 66 .

The controller 38 preferably includes a control program and a downloader program to interface the controller 38 with each digital signal processor 66 . The control program is designed to support initial checkout between each digital signal processor 66 and the controlier 38. The conirol program provides integrated access to the high speed and low speed data buses 36 . The downloader program supports the interface between each digital signal processor 66 and the controller 38, as well as processor memory upload and dowoload access via the low speed bus. Each of the programs on the controller 38 use a command-line based interactive design to allow a user to enter commands to be carried out by the software. Information gathered by the controller 38 can then be transmitted along a communications network to the bost computer 76 to report fight status information. The controller $\mathbf{3 8}$ assesses the integrity of the signals received from each digital signal processor 66 . The controller 38 additionally sends commands to each digital signal processor 66 , instructing each processor as to when a parameter measurement should be taken and verifying the health stalus of each module 22 .

In accordance with another feature of the invention, in exemplary metbod of measuring pbysical parameters about the surface of an object, such as the airframe of an aircraft 12, using a multisensor system 10 as described above is provided. During the operation of the aircraft 12, the first sensor $\mathbf{4 2}$ of each segment-mounted module 22 generates a first analog signal representative of a first preselected physical parameter, such as static pressure, while the second sensor 44 generates a second analog signal representative of a second preselected physical parameter, such as absolute temperature. Afier suitable conditioning and amplification in the analog signal-conditioning processor 60 , the first and second analog signals are digitally-converted in the analogto-digital converter 64 and supplied to the digital signal processor 66 on the module 22 .

The digital signal processor 66 analyzes the first and 35 second digital signals to generate a third digital signal based on first and second sigoals. By way of example only, in the exemplary method, the second (temperature) signal is used to normalize the first (static pressure) signal to thereby account for temperature effects on the first sensor 42 . The 40 digital signal processor 66 transmits the digital third signal, along with the specific processor node address identifying its respective module 22, to the remotely-located controller 38 along the high speed electrically-conductive data bus 36 .

Additionally, digital sigaal processor 66 can transmit data 45 taken from sensors 42,44 , or otber sensors resident on moctule 22 as described above, which may be combined through a predetermined method to generate additional digital sigaals. These additional signals may preferably be communicated to controller 38 on separate channels in tite 50 data bus 36.

In accordance with the invention, in the exemplary method, the controller 38 sends instructions to each digital signal processor 66 along the data bus 36 to transmit the third digital signal generated by the digital sigual processor 5566 of each module 22. The controller 38 collects the thus-transmitted digital third signals, as well as the specific node address of each digital signal processor 66 . The controller 38 analyzes the collected third signals based on a predetermined set of instruetions. The controller 38 then 60 forwards the data from the signals on to a bost computer 76 tbrough the network capable application processor 74 in a communication network to provide flight status information during operation of the aircraft 12. Alternatively, the controller 38 may be configured to act as a host computer to os adalyze tbe flight status information during the operation of the aircraft 12. Additionally, the controller 38 may collect 150 
such data in suitable memory (not shown), to be downloaded by aircraft maintenance support personnel after completion of the flight. The controller 38 may also transmit the collected physical parameter data via a wireless interface to the ground.

While embodiments of the invention bave been illustrated and described, it is not intended that these embodiments illustrate and describe all possible forms of the invention. Rather, the words used in the specification are words of description rather than limitation, and it is understood that various changes may be made without departing from the spirit and scope of the invention. For example, while the invention has been described in the context of an aircraft's flight data survey, it will be appreciated that the multisensor system 10 may be used in testing systems for other modes of transportation, including measuring physical parameters about the rotors of a helicopter, aboul the bull of a boat or submarine, or about the body of an automobile. Additionally, while each module 22 on a given belt segment is illustrated in the Drawings as completely overlying the lape carrier 20 , the invention contemplates use of belt segments 18 whose parameter-sensing modules 22 extend, for example, laterally beyond the confines of the tape carrier 20 . Thus, for example, where one of the physical parameters to be measured with the multisensor system is mechanical strain, the MEMS 46 may preferably extend laterally beyond the tape carrier 20 to thereby mechanically isolate the modulemounted sensors from the tape carrier 20 and, hence, improve the sensitivity of the multisensor system.

What is claimed is:

1. A multisensor system for measuring physical parameters at a plurality of discrete locations about a surface of an object, the system comprising:

at least one belt segment, wherein each belt segment includes an electrically-conductive data bus that is in communication with the data bus of another belt seg- 35 ment;

a plurality of parameter-sensing modules mounted at a plurality of positions on each belt segment corresponding to the discrete locations, each module including a first sensor for generating a first signal representative of 40 a first one of the physical parameters, a second sensor for generating a second signal representative of a second one of the physical parameters, and a processor, in electrical communication with the data bus, receiving the first and second signals and generating a third signal based on the first and second signals, wherein the third signal is a digital signal; and

a controller connected to the data bus for selectively receiving the third signal from the processor of each of the modules.

2. The system of claim 1 , wherein the first sensors of a given belt segment define a one-dimensional sensing array.

3. The system of claim 1 , wherein the first sensor and the second sensor are electromechanical devices which share a common substrate.

4. The system of claim 2, wherein the first one of the physical parameters is stalic pressure.

5. The system of claim 4 , wherein the first one of the physical parameters is static pressure measured in the range of 0 to about 15 psia.

6. The system of claim 1 , wherein the second one of the physical parameters is absolute temperature.

7. The system of claim 6, wherein the second one of the physical parameters is absolute temperature in the range of about $-50^{\circ} \mathrm{C}$. 10 about $85^{\circ} \mathrm{C}$.

8. The system of claim 1 , including a connector for physically interconnecting one belt segment to another belt segment, wherein the connector forms an electrical connection between the data buses of the one belt segmenl and the other belt segment.

9. The system of claim 1 , wherein each belt segment 5 further includes a thin, elongate, fexible substrate.

10. The system of claim 9 , wherein the substrate include a plurality of wiring traces defining a portion of the data bus.

11. The system of claim 9, further including a protective coating overlying the substrate and at least a portion of each module.

12. The system of claim 11 , wherein the coating defines an aerodynamic surface on the bell segment.

13. The system of claim 1, wherein the processor generates at least one additional digital signal based on the first and second signals.

14. The system of claim 1, wherein the third signal is transmitted over a first channel in the data bus, and the additional signal is transmitted over a second channel in the data bus.

15. A multisensor system for measuring physical parameters of flight data at a plurality of discrete locations about a surface of an aircraft, the system comprising:

at least two belt segments, wherein each belt segment includes a connecting portion at each end of the segment to interconnect the belt segments, wherein each belt segment includes an electrically-conductive data bus that is in communication with the data bus of another belt segment;

a plurality of modules disposed on each of the belt segments, wherein each module includes a first sensor for generating a first signal representative of a first one of the physical parameters, a second sensor for generating a second signal representative of a second one of the physical parameters, and a processor receiving the first and second signais and generating a third, digital signal based on the first and second signals, the processor being in electrical communication with the data bus;

a remote controller connected to the data bus for receiving the third signal generated by the processor of each of the plurality of modules.

16. The system of claim 15, wherein the first sensors of the modules of the at least two belt segments define in a two-dimensional sensing array.

17. The system of claim 16, wherein each belt segment is parallel to an adjacent belt segment when disposed on the aircraft in a two-dimensional array.

18. The system of claim 15 , wherein the first sensor and the second sensor are electromechanical devices which stitre so a common substrate.

19. The system of claim 15 , wherein the first sensors are disposed in a one-dimensional array on each belt segment.

20. The system of claim 15, wherein the first one of the physical parameters is static pressure in the range of 0 to 55 about 15 psia.

21. The system of claim 15 , wherein the second one of the physical paramelers is absolute temperature in the range of about $-50^{\circ} \mathrm{C}$. 10 about $85^{\circ} \mathrm{C}$.

22. The system of claim 15 , including a connector for 60 interconnecting each belt segment to another belt segment, wherein the connector forms an electrical connection between the data buses of adjacent beil segments.

23. The system of claim 15 , further including a protective coating overlying each interconnected belt segment and at 65 least a portion of each module.

24. The system of claim 23 , wherein the coating defines
aetodynamic surface on the interconnected belt segments. 151 
25. A method of measuring physical paramelers of fligbt data at a plurality of discrete locations about a surface of an aircraft, the method comprising:

generating, at each of the plurality of discrete locations, a first signal representative of a first one of the physical 5 parameters;

generating, for each of the discrele locations, a second signal representative of a second one of the physical parameters;

generating, at each of the discrete locations, a third signal based on the first and second signals;

transmitting the third signal generated at each of the discrete locations along an electrically-conductive bidirectional data bus; and

collecting the third signal with a remote controller connected to the data bus.
26. The method of claim 25 , wherein the step of collecting the third signal with a remote controller further comprises the step of analyzing the third signal with the remote controller to provide fight status information.

27. The method of claim 25, wherein the slep of generating the first signal representative of the first physical parameter comprises the step of measuring static pressure in the range of 0 to about 15 psia.

28. The metbod of claim 25, where in the step of gener10 ating the second signal representative of the second physical parameters comprises the step of measuring absolute temperature in the range of about $-50^{\circ} \mathrm{C}$. to about $85^{\circ} \mathrm{C}$.

29. The method of claim 25 , wherein the collecting step includes calling for transmission of the third sigaal gener15 ated at each of the discrete locations. 


\section{MISSION \\ OF \\ AFRL/INFORMATION DIRECTORATE (IF)}

The advancement and application of Information Systems Science and Technology to meet Air Force unique requirements for

Information Dominance and its transition to aerospace systems to meet Air Force needs. 